\title{
Computational Modelling for the Optical Properties of Dye Molecules Adsorbed onto Metallic Nanoparticles
}

By

Chhayly Tang

\author{
A thesis \\ submitted to Victoria University of Wellington \\ in fulfilment of the requirements for the degree of \\ Doctor of Philosophy \\ in Physics
}

Victoria University of Wellington

2021 



\section{Abstract}

The study of light scattering by particles has become fundamental and applied interests in the fields of chemistry, biology, and most importantly in physics. In this context, this thesis focuses on understanding the optical properties of dye layers adsorbed onto metallic nanoparticles (NP), which is essential for interpreting the results of plasmon-dye coupling experiments. To model such a system, Mie theory is often used to solve for the exact solution to Maxwell's equations for spherical homogeneous and isotropic coated NP. The effects of the NP's plasmon resonances on the optical properties of the adsorbed dye layer have been predicted using an effective medium model, where the dye-layer is treated as an isotropic layer with an effective dielectric function accounting for the dye resonance. However, this isotropic shell model is inadequate as it cannot account for the dye surface concentration and the anisotropy of the optical response of the dye layer.

In this thesis, we introduce anisotropic effects within Mie theory and develop microscopic models to define effective dielectric functions which explicitly include the dye-concentration effect in the shell model. Combining anisotropic Mie theory with a concentration-dependent effective shell model allows us to form new theoretical tools to model the optical properties of adsorbed dye layers on metallic NPs of spherical shape. With this new refined effective medium model, we are then able to study shell models for elongated particles beyond the quasi-static approximation. This is implemented using the finite element method (FEM) to numerically solve Maxwell's equations. The FEM implementation is then used to investigate how the NP's plasmon resonance can be affected by the dye's orientation and location on the NP's surface. We show that the orientation and location of the dye molecules on the NP determine how strongly the plasmon resonance is shifted.

The results of this work will improve our ability to accurately model the optical properties of anisotropic molecules adsorbed on metallic NPs. This is important in a number of applications including the development of localised surface plasmon 
resonance (LSPR) sensing and the design of plasmonic devices. 


\section{Acknowledgements}

The journey from the beginning toward the completion of this $\mathrm{PhD}$ was not an easy path to take. However, the support from great people in the Raman group has helped this $\mathrm{PhD}$ project come along nicely and bring it to its final stage. Without the supports from the team in the projects, this thesis would not be completed.

First of all, I would like to thank my supervisors Eric C. Le Ru and Baptiste Auguié, who have shown that you can be determined to achieve academic goals. Their support (being available, approachable and patient) has been very inspiring throughout the whole three years of my PhD. Besides being awesome supervisors, they have contributed a large amount to the success of the work presented in this thesis and I will always be in their debt.

I would also like to thank my colleagues within the Raman group: Matt Majic, Walter Somerville, William Hewett, Xiaohan Chen, Johan Grand and Adam Francis. In particular, a special thank to Adam Francis, who has always been discussing and exchanging general knowledge and ideas in physics, and his generous help with the formatting of this thesis.

I am grateful to the MacDiarmid Institute for funding this $\mathrm{PhD}$ research. I am also thankful to various people in the school of chemical and physical sciences (SCPS), especially, Ben Ruck, Natalie Plank, John Lekner, Joyce Lan, Gabrielle Holmes, and Anne Shepherd.

I would like to offer special thanks to the Strudwick family for their support and to thank for my dearest friends Jenny, Harini, Torrance, and Ravy for being very supportive in many ways. 



\section{List of publications}

1. C. Tang, B. Auguié, and E. C. Le Ru, "Modeling molecular orientation effects in dye-coated nanostructures using a thin-shell approximation of Mie theory for radially anisotropic media." ACS Photonics, 5(12):5002-5009, 2018.

2. C. Tang, B. Auguié, and E. C. Le Ru, "Refined effective-medium model for the optical properties of nanoparticles coated with anisotropic molecules." Phys. Rev. B, 103(8):085436, 2021.

3. C. Tang, B. Auguié, and E. C. Le Ru, "Thin-shell approximation of Mie theory for a thin anisotropic layer spaced away from a spherical core: Application to dye-coated nanostructures." Phys. Rev. A, in press, 2021. 
Theoretical assumptions about efficiency in cow milk production: A biologist and a chemist start to discuss about the proteins in cow's milk and chemical uses for cows to efficiently produce more milk. Suddenly, a physicist responds "Let us assume a cow is a sphere, uniformly emitting milk in all directions...." 


\section{Contents}

Abstract $\quad$ i

Acknowledgements

$\begin{array}{ll}\text { List of publications } & \text { v }\end{array}$

1 Introduction $\quad 1$

1.1 Background ......................... 1

1.2 Motivation ...................... 2

1.3 Goals and Outline . . . . . . . . . . . . . . 5

2 Mie Theory and Coupled-Dipole Model $\quad 7$

2.1 Mie Theory . . . . . . . . . . . . . . . . . . . . 7

2.1.1 The Maxwell and Helmholtz Equations in Spherical Coordinates 7

2.1.2 Vector Spherical Harmonics (VSHs) . . . . . . . . . . . 8

2.1.3 Expansion of Field Solutions in VSHs . . . . . . . . . . . . 10

2.1.4 Scattering by a Sphere . . . . . . . . . . . . . . . 11

2.1.5 Scattering by a Coated Sphere (Core-Shell System) . . . . . . 15

2.2 Coupled Dipole Theory or Model $(\mathrm{CDM}) \ldots \ldots$. . . . . . . . . 18

2.2 .1 Green's Tensor . . . . . . . . . . . . . . . . . . . . 19

2.2 .2 Coupled Dipole Equations . . . . . . . . . . . . . 20

2.2.3 Scattering, Absorption, and Extinction . . . . . . . . . . 21

2.3 Generalised Coupled-Dipole Model (GCDM) . . . . . . . . . . . . . . 23

2.4 GCDM Vs. Mie Theory . . . . . . . . . . . . . . . . . . . 24

3 Mie Theory for Anisotropic Shell 25

3.1 Electric and Magnetic Fields for Anisotropic Media . . . . . . . . . . 26

3.1.1 Maxwell's Equations . . . . . . . . . . . . . . . . 26

3.1 .2 Anisotropic VSHs . . . . . . . . . . . . . . . . . . 27

3.2 Scattering by Anisotropic Shell/Isotropic Core . . . . . . . . . . . . . 28 
3.2.1 Boundary Conditions and The Susceptibilities . . . . . . . . 29

3.2.2 Thin Anisotropic Shell Approximations . . . . . . . . . . . . . 30

3.3 Validation of TASA Model and Application . . . . . . . . . . . 34

3.3.1 Microscopic Model for an Anisotropic Layer of Molecules Adsorbed on a Metal Sphere . . . . . . . . . . . . . . 35

3.3.2 Effective Medium Model (Effective Dielectric Function) . . . . 37

3.3.3 Comparison Between Full Anisotropic Mie and TASA . . . . . 38

3.4 Summary and Outlook . . . . . . . . . . . . . . . . . . . . . 40

4 Refined Model for Effective Dielectric Functions in Anisotropic Media 43

4.1 The Dielectric Function(DF) for Molecular Monolayer . . . . . . . . . 44

4.1 .1 Isotropic DF for 3D bulk . . . . . . . . . . . . . . . . . . 44

4.1.2 Anisotropic DF for 2D Planar Array of Dipoles in Vacuum . . 47

4.1.3 Anisotropic DF for 2D Planar Array of Dipoles in a Medium . 49

4.2 Anisotropic Fresnel Equations . . . . . . . . . . . . . . . . . . 52

4.2.1 Wave-Vector $\mathbf{k}$ in Anisotropic Media . . . . . . . . . . . . . 52

4.2 .2 Electric and Magnetic Fields . . . . . . . . . . . . . . . . 53

4.2.3 Absorption Coefficients and Boundary Conditions . . . . . . . 54

4.3 An Improved Microscopic Model with Radiative Correction Term . . 56

4.3 .1 In Vacuum . . . . . . . . . . . . . . . . . . . . . 56

4.3 .2 In Medium . . . . . . . . . . . . . . . . . . . . 57

4.3.3 Anisotropic EMM planar vs MM predictions . . . . . . . . 58

4.4 Implementing Anisotropic DF into Anisotropic Mie Theory . . . . . . 58

4.4.1 Comparison to GCDM . . . . . . . . . . . . . 60

4.4.2 $\mathbf{M}_{\mathrm{loc}}$ as a Function of Distance Away from the Sphere . . . . . 60

4.4 .3 Two-Layer System . . . . . . . . . . . . . . . . . . 65

4.5 Self-Reflected Field or Image-Dipole Effects . . . . . . . . . . . . . . 65

4.5.1 View from Microscopic Model: Effective Polarisability with Image-Dipole Effect . . . . . . . . . . . . . . . . . . 66

4.5.2 Anisotropic EMM with Image-Dipole Effect . . . . . . . . . . 67

4.5.3 A Complete EMM vs GCDM . . . . . . . . . . . . . . . 70

4.6 Conclusion . . . . . . . . . . . . . . . . . . . 71

5 Anisotropic EMM Shells on Non-Spherical Particles 73

5.1 General Considerations in COMSOL . . . . . . . . . . . . . . . . . 74

5.1 .1 FEM Model Description . . . . . . . . . . . . . . . 74

5.1 .2 Electric Fields . . . . . . . . . . . . . . . . . 75 
5.1.3 Absorption, Scattering, and Extinction Cross-Sections . . . . . 75

5.1.4 Single Spherical Particle . . . . . . . . . . . . . . . 76

5.1.5 Single Spheroidal Particle . . . . . . . . . . . . . . . . . 80

5.2 Scattering by a Core-Shell System . . . . . . . . . . . . . . . . 82

5.2.1 Spherical: Isotropic Core-Shell . . . . . . . . . . . . . . 83

5.2.2 Spherical: Isotropic Core-Anisotropic Shell . . . . . . . . . . . 83

5.2.3 Spheroidal: Isotropic Core-Anisotropic Shell . . . . . . . . . . 87

5.3 Conclusion . . . . . . . . . . . . . . . . . . . . . . . 91

6 The Effects of Anisotropic Molecules on the Nanoparticles' Plasmon Resonance $\quad 93$

6.1 Refractive Index Dependence . . . . . . . . . . . . . . . . . . . . . 94

6.2 Dye's Properties Dependence . . . . . . . . . . . . . . . . . . . . . . 97

6.2.1 Dye's Polarisability Dependence . . . . . . . . . . . . . . . . 97

6.2.2 Dye Molecules Adsorbed on the Ag Sphere . . . . . . . . . . . 98

6.2.3 Dye's Orientation Dependence . . . . . . . . . . . . . . . . 100

6.3 Comparisons Between Mie Theory, GCDM, and COMSOL . . . . . . 102

6.3.1 Full Core/Shell: Mie vs GCDM . . . . . . . . . . . . . . 103

6.3.2 Full Core/shell: Mie vs. COMSOL . . . . . . . . . . . 103

6.4 Dye's Location Dependence . . . . . . . . . . . . . . . . . . . . . 106

6.4.1 Spherical Caps on Ag-NP . . . . . . . . . . . . . . . . 108

6.4.2 Partial Spherical Shell around Ag-NP . . . . . . . . . . . . . . 108

6.5 Conclusion and Future Work . . . . . . . . . . . . . . . . . . . . . . . 109

$\begin{array}{lll}7 & \text { Conclusion and Outlook } & 111\end{array}$

$\begin{array}{ll}\text { A Bessel Function Series Expressions } & 115\end{array}$

$\begin{array}{ll}\text { B Associated Legendre Function } & 117\end{array}$

$\begin{array}{lr}\text { C Orthogonal Properties of VSHs } & 119\end{array}$

$\begin{array}{lr}\text { D Solutions for 2-Layer System } & 121\end{array}$

D.1 Full Anisotropic Mie Solutions . . . . . . . . . . . . . . . . . . . . . 121

D.2 TASA Solutions . . . . . . . . . . . . . . . . . 123

$\begin{array}{ll}\text { Bibliography } & 125\end{array}$ 



\section{Introduction}

\subsection{Background}

The interaction between light and matter is central to a fundamental understanding of physics and nano-science (also for other various topics, e.g. Earth's climate research, cosmology, and astrophysics). These interactions can be observed from a light scattering process, where the light is shone onto a substrate or an object. Its optical properties can be determined by the reflection, absorption, and transmission signals during the process.

The field of light scattering has a long history and it has been applied in a myriad of contexts: medicines development and drug discovery $[1,2]$, modelling the optical properties of cosmic dust grain [3], or optical spectroscopy [4, 5]. It has also led to the new field of plasmonics in physics, which is the study of the optical properties of noble metal (e.g. silver and gold in particular) when incident light interacts with free electrons in metallic nanoparticles. The field of plasmonics has immensely advanced over the years and is spreading more and more into the area of sensor technology $[6,7,8,9]$. This is thanks to the unique interaction of light with noble metals [10].

The study of light/matter interactions (at nanometer length-scales, known as Nanophotonics) is commonly applied to provide a fundamental understanding of physics and nanoscience. Surface plasmon resonance is the resonant oscillation of conducting electrons at the surface of a metal stimulated by incident light. The interactions between the surface plasmon resonance of the metallic nanostructure and the electronic resonance of the adsorbed molecules give rise to very interesting phenomena, such as: sub-wavelength manipulation (nano-antennas $[11,12]$, or meta-materials $[13,14]$ ), surface-enhanced Raman spectroscopy, and fluorescence $[15,16,17,18,19,20]$, plasmonic-enhanced light applications on improving solar cells $[21,22]$, molecular-plasmonic resonance coupling [23, 24, 25, 26], and sensing 
$[6,7]$. Most of these applications are supported by the fact that the optical (electronic) absorption of the molecules on the surface of metallic NPs is greatly enhanced (surface-enhanced absorption [20, 27]).

In the study of light scattering by colloids (NPs) in solutions of dye-molecules, many experiments have been carried out to investigate the interactions between the plasmon resonance of metallic films (or NPs) and the electronic resonance of dyemolecules adsorbed on their surfaces $[23,24,25,28,29$, 30], with a recent surge of interest in the strong-coupling regimes $[31,32,33]$. For dye-molecules adsorbed on metal colloids, previous experimental results have evidenced that different species of molecules experience different enhancement, quenching, spectral modification or shift in their absorption spectrum [34].

\subsection{Motivation}

The metallic NPs can act as resonant antennas for light, which amplify the electromagnetic field that result in significant enhancements of the rate of excitation and re-emission of light in close proximity to the metal surface. When dye molecules are adsorbed onto metallic NPs, their optical properties were shown to be remarkably influenced by the NPs [34]. In relation to that, Fig. 1.1 shows experimental results for Rhodamine $6 \mathrm{G}$ on silver colloids. It suggests that by increasing the dye's coverage from 2.5 to $20 \mathrm{nM}$ [nano-Molar], spectral modifications and shifts (blue-shift) are observed in the absorbance spectrum. In that study, a modified Clausius Mossotti relation was used to derive the isotropic dielectric function, which was then applied to theoretically predict the experimental outcomes. However, that approach loses the quantitative connection between the dielectric function of the shell medium and the dye's concentration, which is significant at higher concentrations when the dyedye interactions can no longer be neglected. To account for the dye-dye interactions, further theoretical studies are therefore required to provide more understanding of the intrinsic effect of nanoparticles on the optical response of the adsorbed molecules.

The results evidenced in Ref. [34] are the primary motivation for this project. We want to apply our understanding of the electromagnetic scattering of light to elucidate these effects that are experienced by the adsorbed molecules. In reference to that, a recent publication on optical absorption of dye-molecules in a spherical shell geometry [35] explained the dye-dye (dipole-dipole) interactions by using coupled-dipole theory [36], in which the dye-molecules are treated as dipoles. 


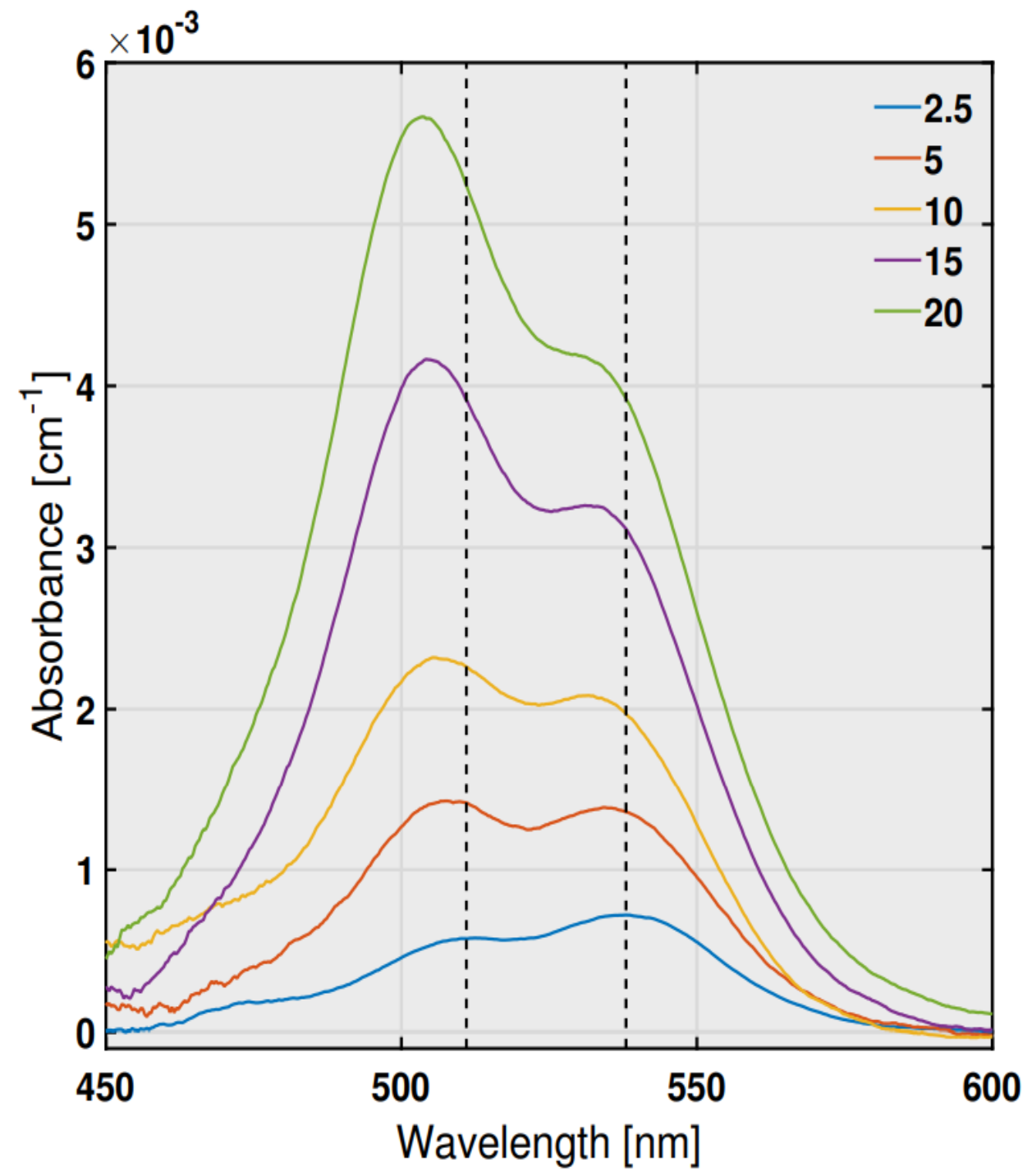

Figure 1.1: Concentration dependence of the differential absorption spectrum of Rhodamine $6 \mathrm{G}$ adsorbed to $\mathrm{Ag}$ colloids measured at 2.5, 5, 10, 15 and $20 \mathrm{nM}$. The black lines indicate the peak transitions for the lowest concentration $(2.5 \mathrm{nM})$ at $\sim 538 \mathrm{~nm}$ and $\sim 511 \mathrm{~nm}$. As the concentration increases to $20 \mathrm{nM}$ there is a clear blue shift in the peak positions along with a change in relative intensities of the two peaks. Taken from Figure S8 in the supplementary information of Ref. [34]. 
The results show that the optical absorption spectra change substantially with dyeconcentration, and they also depend on the orientations of the molecules. It was evidenced that different spectral shifts are observed for different orientation of the dye-molecules. As the dye's concentration increased, a blue-shift and red-shift in the absorption cross-section spectra are observed for dyes pointing radially and tangentially to the surface of the sphere, respectively. The study in Ref. [35] was later extended to molecules adsorbed on a nanoparticle. This extension was done by including the effect of the nanoparticle via solving Maxwell's equations for the interactions between each dipole and the nanoparticle, which we will refer to in this thesis as the generalised coupled-dipole model, GCDM [37]. It can be used to study a rich array of inter-related parameters that will affect the optical properties: molecular orientation, dye-dye interactions, image-dipole effect (or self-reflected field), and spatial distribution of adsorbates.

Using the theoretical modelling and explanation in these two publications, the results evidenced in ref. [34] can be well predicted, theoretically. This GCDM is the most refined model to provide the solutions to the electromagnetic interactions between the dye-molecules and the metallic nanoparticle, in the dye-coated NP system. However, the GCDM has a major shortcoming to it which is the computing requirements. To model a $30 \mathrm{~nm}$ radius nanosphere with a $1 \mathrm{~nm}^{-2}$ coverage of dye-molecules, the GCDM requires more than $10^{4}$ polarisable dipoles. Even on a high-end PC, this may take from a few hours to a day to compute a spectrum (of 100 wavelength points), which is very time-consuming for multi-parameter studies. Therefore, the aim of this $\mathrm{PhD}$ project is to develop and validate an improved effective medium model with comparable predictive power as the GCDM, without the high cost and complexity from the computational perspective.

In the context of the electromagnetic theory of light scattering by finite objects (in spherical coordinates), one can use Mie theory [38] to provide an analytical and rigorous solution to Maxwell's equations for spheres (or stratified spheres). It is commonly applied to isotropic media, where the dielectric functions of the media are the same in all directions. However, there are many realistic situations where the isotropic assumption is not satisfied, so the concepts of anisotropic media were developed, for example, in the context of the refraction/reflection at interfaces [39]. The extension of Mie theory to anisotropic media was introduced almost five decades ago by Roth and Dignam [40] and has been used to study the optical properties of surface plasmon resonance of small inorganic and organic metal particles [41] and 
other influence of anisotropy on plasmon resonances $[42,43]$. Although the theory of anisotropic Mie scattering can be used/applied to model many experimental results, it is still not perfect due to some factors that have not been considered, for example, the dielectric functions for the anisotropic media. Combining anisotropic Mie theory with the appropriate dielectric functions for the anisotropic media will form a new refined effective medium model (EMM). With this refined EMM, we hope to show that it can be used to provide theoretical results of comparable predictive power as the GCDM and to reduce the simulation time, which will be a lot quicker than the GCDM.

\subsection{Goals and Outline}

In this project, we will first re-visit the theory of anisotropic Mie scattering. The effect of the different molecular orientations of the dye-molecules can be accounted for by using solutions from the anisotropic Mie theory. However, the effects of dyedye interactions, self-reflected fields or image-dipole effect, and spatial distribution of adsorbates still have to be included. We can capture these effects in the dielectric functions of the anisotropic media. The dielectric functions for a $2 \mathrm{D}$ anisotropic medium, which include the effects of dye-dye interactions (in a context of 2D planar array of dipoles), have already been derived by Dignam and Moskovits [44] and further developed by Bagchi et al. [45, 46] to include image-dipole effect or self-reflected field. These collections of work, however, were for dipoles in vacuum (dielectric constant $\varepsilon_{\mathrm{m}}=1$ ), which is not relevant to most of the experimental conditions where particles are suspended in water or other solvents with $\varepsilon_{\mathrm{m}} \neq 1$. Therefore, our first goal is to generalise the results from these previous works to the case where the dipoles are embedded in an isotropic and non-absorbing medium with $\varepsilon_{\mathrm{m}} \neq 1$.

The second goal will be to combine the results of the new effective dielectric functions with the anisotropic Mie theory to form a new refined effective medium model. The new model will then be compared to the solutions from the GCDM of the same problem, dye-molecules surrounding a metallic nanoparticle. The validity of this improved effective medium model was successfully proven in our recent publication [47]. The main advantage of this refined EMM is that its simulation times are less than five minutes to compute the far-field properties of the core-shell system for three different orientations of the dye molecules with dye's surface coverage of $\left(0.01,0.2: 0.2: 1.2 \mathrm{~nm}^{2}\right)$ on a $14 \mathrm{~nm}$ silver sphere. This is much faster than the GCDM simulation, which could take approximately one whole day to compute. 
Since light scattering is the main topic of this thesis, we begin Chapter 2 by introducing Mie theory and its solutions to the light scattering problem by a spherical core-shell system, and the coupled-dipoles model (CDM) and its generalisation for polarisable dipoles surrounding a spherical core. Chapter 3 presents the extension of solutions of Mie theory from isotropic to anisotropic response in the shell, the effective dielectric functions (at low coverage of molecules), and introduce the thin anisotropic shell approximation. The work in Chapter 3 was published in Ref. [48].

A refined effective medium model is presented in Chapter 4, which shows the derivations of the effective dielectric functions for 2D planar array of dipoles in a medium of permittivity $\varepsilon_{\mathrm{m}}$. These effective dielectric functions can be validated by combining them with anisotropic Fresnel equations to study the optical properties of 2D layer of dye molecules and by comparing its predictions with an improved microscopic model. The results also show that the new refined effective medium model (effective dielectric functions with anisotropic Mie theory) gives very good agreement with the results from the GCDM. The results presented in Chapter 4 were published in Ref. [47].

The study of light scattering by a core-shell system can be extended from particles of spherical shape to those of non-spherical shape. This extension is presented in Chapter 5, where the practical implementation of the finite element method into COMSOL simulation is demonstrated. Chapter 6 presents the results of how the dye-molecules may affect the plasmon resonance. They demonstrate how the plasmon resonances of the NPs are affected by the properties of the dye molecules, such as: their molecular orientations, polarisability, and the importance of their locations on the nanoparticle's surface.

Finally, Chapter 7 presents a brief conclusion and discussion of the importance of the results and future research that might arise from this work. 


\section{Mie Theory and Coupled-Dipole Model}

Many approaches have been used to solve the light scattering problem by particles, such as Discrete Dipole Approximation (DDA) [36], Mie theory [38], T-matrix [49], Finite Element Method (FEM) [50], Finite-Difference Time-Domain (FDTD) [51]. These approaches solve for the electric and magnetic fields that satisfy the Maxwell equations, with appropriate boundary conditions. Some of these can be extended to the study of the optical properties of a core-shell system (or coated particle) if an effective medium model is used to determine the dielectric function of the molecular layer. The latter may represent a nanoparticle surrounded by molecules, in the microscopic point of view.

This chapter begins with the solutions of Mie theory for both a single particle and a coated particle. It then follows with the solutions of the light scattering problem by a shell of molecules from a microscopic point of view, called coupled dipole model (CDM). Furthermore, we present how to generalise the CDM to include a core (particle) surrounded by a shell of molecules.

\subsection{Mie Theory}

In this section, we will re-derive Mie theory solution for light scattering problem by a sphere with notations that will be used throughout this thesis. For more details and references, the solution is treated comprehensively in the textbook by Bohren and Huffman [38].

\subsubsection{The Maxwell and Helmholtz Equations in Spherical Coordi- nates}

We begin by considering the Maxwell equations (for complex fields with time-dependent denoted as $e^{-i \omega t}$ ) for a region of no charge and current (whose optical properties are 
described by the frequency-dependent dielectric function, $\varepsilon(\omega)$ ), which take the form:

$$
\begin{array}{ll}
\nabla \cdot \mathbf{E}=0, & \nabla \cdot \mathbf{H}=0, \\
\nabla \times \mathbf{E}=i \omega \mu \mathbf{H}, & \nabla \times \mathbf{H}=-i \omega \varepsilon \mathbf{E} .
\end{array}
$$

Where, $\mathbf{E}$ and $\mathbf{H}$ are the electric and magnetic fields, $\omega$ is the frequency, $\mu$ and $\varepsilon$ are the permeability and permittivity of the medium, respectively. Using these equations, one can show that (for a linear, isotropic, homogeneous medium) the electric field, E, and magnetic fields, H, must satisfy the vector Helmholtz equation:

$$
\begin{aligned}
& \nabla^{2} \mathbf{E}+k^{2} \mathbf{E}=\mathbf{0}, \\
& \nabla^{2} \mathbf{H}+k^{2} \mathbf{H}=\mathbf{0},
\end{aligned}
$$

where $k$ is the wave-vector and can be defined as $k^{2}=\omega^{2} \mu \varepsilon(\omega)$, and the Laplacian operator $\nabla^{2}$ may be written (in spherical coordinates) as:

$$
\nabla^{2}=\frac{1}{r^{2}} \frac{\partial}{\partial r}\left(r^{2} \frac{\partial}{\partial r}\right)+\frac{1}{r^{2} \sin \theta} \frac{\partial}{\partial \theta}\left(\sin \theta \frac{\partial}{\partial \theta}\right)+\frac{1}{r^{2} \sin ^{2} \theta} \frac{\partial^{2}}{\partial \phi^{2}}
$$

\subsubsection{Vector Spherical Harmonics (VSHs)}

In order to solve the vector Helmholtz equation, we need to construct a vector function $\mathbf{M}$ from a scalar function $f(r, \theta, \phi)$ and a radius vector $\mathbf{r}$ such that:

$$
\mathbf{M}=\nabla \times(\mathbf{r} f)=\nabla f \times \mathbf{r} \quad \text { so, } \quad \nabla \cdot \mathbf{M}=0 .
$$

Then by using vector calculus identities of $[\nabla \times(\mathbf{A} \times \mathbf{B})$, and $\nabla(\mathbf{A} \cdot \mathbf{B})]$, we get:

$$
\nabla^{2} \mathbf{M}+k^{2} \mathbf{M}=\nabla \times\left[\mathbf{r}\left(\nabla^{2} f+k^{2} f\right)\right] .
$$

Therefore, $\mathbf{M}$ satisfies the vector Helmholtz equation if $f$ is a solution to the scalar Helmholtz equation, which is:

$$
\nabla^{2} f+k^{2} f=0
$$

We then use the vector $\mathbf{M}$ to construct another vector $\mathbf{N}$ as:

$$
\mathbf{N}=\frac{\nabla \times \mathbf{M}}{k}, \quad \rightarrow \quad \nabla \times \mathbf{N}=k \mathbf{M}
$$

$\mathbf{N}$ also satisfies the vector Helmholtz equation and is divergence-free. The vectors $\mathbf{M}$ and $\mathbf{N}$ have all the required properties of an electromagnetic field. They satisfy 
the vector Helmholtz equation and are divergence-free. So, the electromagnetic fields $\mathbf{E}$ and $\mathbf{H}$ can be represented by linear combinations of the vectors $\mathbf{M}$ and $\mathbf{N}$. The function $f$ is known as a generating function for the vector harmonics, and $\mathbf{r}$ is the radius vector in spherical coordinates. Therefore, instead of trying to solve for the solution of the vector Helmholtz equations, the problem now is simplified to solving the scalar Helmholtz equation (Eq. 2.6), which can also be expressed as:

$$
\frac{1}{r^{2}} \frac{\partial}{\partial r}\left(r^{2} \frac{\partial f}{\partial r}\right)+\frac{1}{r^{2} \sin \theta} \frac{\partial}{\partial \theta}\left(\sin \theta \frac{\partial f}{\partial \theta}\right)+\frac{1}{r^{2} \sin ^{2} \theta} \frac{\partial^{2} f}{\partial \phi^{2}}+k^{2} f=0
$$

To solve this equation, we use separation of variables and seek particular solutions of the form:

$$
f(r, \theta, \phi)=R(r) \Theta(\theta) \Phi(\phi)
$$

By substituting this into Eq. 2.8, it yields the three separated equations:

$$
\begin{aligned}
\frac{d^{2} \Phi}{d \phi^{2}}+m^{2} \Phi & =0, \\
\frac{1}{\sin \theta} \frac{d}{d \theta}\left(\sin \theta \frac{d \Theta}{d \theta}\right)+\left(n(n+1)-\frac{m^{2}}{\sin ^{2} \theta}\right) \Theta & =0, \\
\frac{d}{d r}\left(r^{2} \frac{d R}{d r}\right)+\left[k^{2} r^{2}-n(n+1)\right] R & =0,
\end{aligned}
$$

where $n$ and $m$ are the separated constants. The solutions to the first equation are sinusoids, $e^{i m \phi}$, and it is required that the solution is $2 \pi$-periodic in $\phi$, so $m$ must be an integer. For the second equation, its solutions are the associated Legendre polynomials functions $P_{n}^{m}(\cos \theta)$ [52], as defined in Appendix B. From this, $n$ is required to be also an integer and that $|m| \leq n$. For the third equation, if we use the substitution $(\rho=k r)$, then it may be rewritten as:

$$
\frac{d}{d \rho}\left(\rho^{2} \frac{d R}{d \rho}\right)+\left[\rho^{2}-n(n+1)\right] R=0 .
$$

The solutions to this equation are known to be the spherical Bessel functions of the first kind $j_{n}(\rho)$, the second kind $y_{n}(\rho)$, and linear combinations of these, the first and second kinds of the Hankel functions, $h_{n}^{(1)}(\rho)=j_{n}(\rho)+i y_{n}(\rho)$ and $h_{n}^{(2)}(\rho)=j_{n}(\rho)-i y_{n}(\rho)$, respectively. For now, we will use the notation $z_{n}^{(j)}$ to represent these solutions, where the superscript $(j)$ denotes four different types of the Bessel function, $j=1 \ldots 4$ [i.e., $z_{n}^{(1)}$ corresponds to $j_{n}(\rho), z_{n}^{(2)}$ corresponds to $y_{n}(\rho), z_{n}^{(3)}$ corresponds to $h_{n}^{(1)}(\rho)$, and $z_{n}^{(4)}$ corresponds to $\left.h_{n}^{(2)}(\rho)\right]$. Therefore, the 
vector $\mathbf{M}$ can be found (from Eqs. 2.4 and 2.9 with a normalisation factor $\mathcal{F}_{n m}$ ) as:

$$
\begin{gathered}
\mathbf{M}_{n m}^{(j)}(\rho, \theta, \phi)=\mathcal{F}_{n m} \nabla \times\left(\mathbf{r} e^{i m \phi} P_{n}^{m}(\cos \theta) z_{n}^{(j)}(\rho)\right), \\
\text { with } \quad \mathcal{F}_{n m}=\sqrt{\frac{1}{4 \pi} \frac{2 n+1}{n(n+1)} \frac{(n-m) !}{(n+m) !}}
\end{gathered}
$$

[NB: $\mathbf{M}^{(1)}$ corresponds to the regular solution (finite at the origin), and $\mathbf{M}^{(3)}$ corresponds to outgoing spherical waves.] These also lead to:

$$
\mathbf{N}_{n m}^{(j)}(\rho, \theta, \phi)=\frac{\nabla \times \mathbf{M}_{n m}^{(j)}(\rho, \theta, \phi)}{k},
$$

These vectors $\mathbf{M}$ and $\mathbf{N}$ are called vector spherical harmonics (VSHs), sometimes known as vector-spherical wave-functions (VSWFs). Physically, the indices $n$ and $m$ can be represented as a total angular momentum, and the projection of this momentum along the $z$-axis (in the spherical coordinates), respectively. They may take their values as $0 \leq n<\infty$ and $-n \leq m \leq n$.

\subsubsection{Expansion of Field Solutions in VSHs}

The VSHs satisfy the vector Helmholtz equation, are divergence-free, and form a basis of the solution in the spherical coordinates. So, the fields $\mathbf{E}$ and $\mathbf{H}$ can be expressed as linear combinations of $\mathbf{M}$ and $\mathbf{N}$. It can be shown (as in Ref. [38]) that a general divergence-less solution of the vector Helmholtz equations can be written in spherical coordinates as an infinite series of a complete set of VSHs as:

$$
\mathbf{E}(\mathbf{r})=E_{0} \sum_{n=0}^{\infty} \sum_{m=-n}^{m=n} a_{n m} \mathbf{M}_{n m}^{(j)}(k, \mathbf{r})+b_{n m} \mathbf{N}_{n m}^{(j)}(k, \mathbf{r}),
$$

where $k$ was defined earlier and depends on the optical properties of the medium which are contained in the dielectric function $\varepsilon$. The coefficients $a_{n m}$ and $b_{n m}$ are non-dimensional coefficients and can be complex. $E_{0}$ (possibly complex also) is the amplitude of the electric field (arbitrary at this stage).

The VSHs $\mathbf{M}_{n m}^{(j)}(k, \mathbf{r})$ and $\mathbf{N}_{n m}^{(j)}(k, \mathbf{r})$ can also be called multi-pole fields. They correspond to the electric and magnetic fields created by a given multi-polar distributions of sources at the origin. For example, (for $n=1) \mathbf{N}_{1 m}^{(j)}(k, \mathbf{r})$ corresponds to the electric fields of an electric dipole, and $\mathbf{M}_{1 m}^{(j)}(k, \mathbf{r})$ corresponds to that of a magnetic dipole. For $n>1$, they correspond to the electric fields of higher-order electric and magnetic multipoles (e.g. quadrupole, octopole, etc.). 
Taking the curl of the electric field in Eq. 2.17, the associated magnetic field can be found as

$$
\begin{aligned}
& \mathbf{H}(\mathbf{r})=H_{0} \sum_{n=0}^{\infty} \sum_{m=-n}^{m=n} b_{n m} \mathbf{M}_{n m}^{(j)}(k, \mathbf{r})+a_{n m} \mathbf{N}_{n m}^{(j)}(k, \mathbf{r}), \\
& \text { where } \quad H_{0}=\frac{k}{i \omega \mu} E_{0} .
\end{aligned}
$$

We can now use this expansion formalism to solve EM problems of objects with spherical geometry excited by an incident wave with a given electric field $\mathbf{E}_{\text {Inc }}(\mathbf{r})$ at frequency $\omega$ (or wavelength of $\lambda=2 \pi c / \omega$ ).

\subsubsection{Scattering by a Sphere}

This section is to summarise the Mie solutions for a single (uncoated) spherical particle. We here consider a sphere with radius $a$, whose optical properties are characterised by a local (possibly frequency dependent) dielectric function $\varepsilon_{\text {in }}(\omega)$. It is assumed to be located at the origin $O$ and surrounded by a medium with dielectric function $\varepsilon_{\mathrm{m}}$. The corresponding wave vectors, inside the sphere, and in the embedding medium, are given as:

$$
k_{\text {in }}=\sqrt{\varepsilon_{\text {in }}} \frac{\omega}{c} \quad \text { and } \quad k_{\mathrm{m}}=\sqrt{\varepsilon_{\mathrm{m}}} \frac{\omega}{c} .
$$

In scattering problems where an incident field $\mathbf{E}_{\text {Inc }}$ is imposed externally, then it is convenient to write the field outside the sphere as the combination of the incident and scattered fields, i.e.

$$
\mathbf{E}_{\text {out }}=\mathbf{E}_{\text {Inc }}+\mathbf{E}_{\text {Sca }} \text {. }
$$

We shall now expand these fields, incident field $\mathbf{E}_{\text {Inc }}$, scattered field $\mathbf{E}_{\text {Sca }}$, and the internal field inside the sphere $\mathbf{E}_{\text {in }}$ as the sum of a series of VSHs as it is necessary to express them in spherical coordinates and they have to satisfy the vector Helmholtz equation.

For the incident field, it has to be finite at the origin of the sphere. Therefore, it can be expanded in terms of regular VSHs $\mathbf{M}_{n m}^{(1)}$ and $\mathbf{N}_{n m}^{(1)}{ }^{1}$, which is:

$$
\mathbf{E}_{\text {Inc }}=E_{0} \sum_{n, m} a_{n m} \mathbf{M}_{n m}^{(1)}\left(k_{\mathrm{m}}, \mathbf{r}\right)+b_{n m} \mathbf{N}_{n m}^{(1)}\left(k_{\mathrm{m}}, \mathbf{r}\right)
$$

\footnotetext{
${ }^{1}$ The incident field is finite at the origin, which requires that $j_{n}(\rho)$ is the appropriate spherical Bessel function in the generating function $f$. We reject $y_{n}(\rho)$ in this case because of its misbehaviour at the origin.
} 
where $a_{n m}$ and $b_{n m}$ are known complex coefficients, which depend on the form of incident field, e.g. plane-wave incident or emission of a radiating dipole. Similarly, the internal field is also expanded in terms of $\mathbf{M}_{n m}^{(1)}$ and $\mathbf{N}_{n m}^{(1)}$ as:

$$
\mathbf{E}_{\mathrm{in}}=E_{0} \sum_{n, m} c_{n m} \mathbf{M}_{n m}^{(1)}\left(k_{\mathrm{in}}, \mathbf{r}\right)+d_{n m} \mathbf{N}_{n m}^{(1)}\left(k_{\mathrm{in}}, \mathbf{r}\right)
$$

where $c_{n m}$ and $d_{n m}$ are complex coefficients to be determined. For the magnetic field inside the sphere, we can use Eq. 2.18 with $H_{0}=k_{\text {in }} E_{0} /(i \omega \mu)$.

At infinity $(r \rightarrow \infty)$, the relevant VSHs $\mathbf{M}^{(3)}$ and $\mathbf{N}^{(3)}$ represent radiated electromagnetic fields, with an $e^{i k r} /(k r)$ dependence and no component along $\hat{\mathbf{e}}_{r}$. Since the scattered field behaves as outgoing spherical waves at infinity, it can be expanded in terms of the irregular VSHs $\mathbf{M}_{n m}^{(3)}$ and $\mathbf{N}_{n m}^{(3)}$ and is therefore written as:

$$
\mathbf{E}_{\mathrm{Sca}}=E_{0} \sum_{n, m} p_{n m} \mathbf{M}_{n m}^{(3)}\left(k_{\mathrm{m}}, \mathbf{r}\right)+q_{n m} \mathbf{N}_{n m}^{(3)}\left(k_{\mathrm{m}}, \mathbf{r}\right),
$$

where $c_{n m}$ and $d_{n m}$ are unknown complex coefficients and to be determined. The corresponding magnetic field $\mathbf{H}_{\text {Sca }}$ can then be derived from Eq. 2.18, with $H_{0}=$ $k_{\mathrm{m}} E_{0} /(i \omega \mu)$.

\section{Boundary Conditions and The Susceptibilities}

As we are able to expand the incident, scattered, and internal fields in terms of the VSHs in spherical coordinates, the next step to provide solutions in Mie theory is to consider the boundary conditions on the particle's surface at $r=a$, which are:

$$
\begin{aligned}
& \mathbf{E}_{\text {out }} \cdot \hat{\theta}=\mathbf{E}_{\text {in }} \cdot \hat{\theta} \\
& \mathbf{E}_{\text {out }} \cdot \hat{\phi}=\mathbf{E}_{\text {in }} \cdot \hat{\phi} \\
& \mathbf{H}_{\text {out }} \cdot \hat{\theta}=\mathbf{H}_{\text {in }} \cdot \hat{\theta} \\
& \mathbf{H}_{\text {out }} \cdot \hat{\phi}=\mathbf{H}_{\text {in }} \cdot \hat{\phi} .
\end{aligned}
$$

These boundary conditions arise from continuity of the tangential components of the electric field $\mathbf{E}$ and magnetic field $\mathbf{H}$. Solving these boundary conditions (with the orthogonality properties of $\mathbf{M}$ and $\mathbf{N}^{2}$ ) leads to

$$
\begin{array}{ll}
p_{n m}=\Gamma_{n} a_{n m}, & q_{n m}=\Delta_{n} b_{n m}, \\
c_{n m}=A_{n} a_{n m}, & d_{n m}=B_{n} b_{n m},
\end{array}
$$

\footnotetext{
${ }^{2}$ see Appendix C for more details
} 
where $\Gamma_{n}$ and $\Delta_{n}$ are the scattered field susceptibilities, which are usually referred to magnetic and electric susceptibilities, respectively. $A_{n}$ and $B_{n}$ refer to the internal field susceptibilities. They all can be computed as follows:

$$
\begin{gathered}
\Gamma_{n}=-\frac{\psi_{n}\left(s_{\text {in }} x\right) \psi_{n}^{\prime}(x)-s_{\text {in }} \psi_{n}^{\prime}\left(s_{\text {in }} x\right) \psi_{n}(x)}{\psi_{n}\left(s_{\text {in }} x\right) \xi_{n}^{\prime}(x)-s_{\text {in }} \psi_{n}^{\prime}\left(s_{\text {in }} x\right) \xi_{n}(x)}, \\
\Delta_{n}=-\frac{s_{\text {in }} \psi_{n}\left(s_{\text {in }} x\right) \psi_{n}^{\prime}(x)-\psi_{n}^{\prime}\left(s_{\text {in }} x\right) \psi_{n}(x)}{s_{\text {in }} \psi_{n}\left(s_{\text {in }} x\right) \xi_{n}^{\prime}(x)-\psi_{n}^{\prime}\left(s_{\text {in }} x\right) \xi_{n}(x)}, \\
A_{n}=\frac{s_{\text {in }} \psi_{n}(x) \xi_{n}^{\prime}(x)-s_{\text {in }} \psi_{n}^{\prime}(x) \xi_{n}(x)}{\psi_{n}\left(s_{\text {in }} x\right) \xi_{n}^{\prime}(x)-s_{\text {in }} \psi_{n}^{\prime}\left(s_{\text {in }} x\right) \xi_{n}(x)}, \\
B_{n}=\frac{s_{\text {in }} \psi_{n}(x) \xi_{n}^{\prime}(x)-s_{\text {in }} \psi_{n}^{\prime}(x) \xi_{n}(x)}{s_{\text {in }} \psi_{n}\left(s_{\text {in }} x\right) \xi_{n}^{\prime}(x)-\psi_{n}^{\prime}\left(s_{\text {in }} x\right) \xi_{n}(x)} .
\end{gathered}
$$

These susceptibilities are written in terms of the Riccati-Bessel functions $\psi_{n}(x)=$ $x j_{n}(x)$ and $\xi_{n}(x)=x h_{n}^{(1)}(x)$, the relative refractive index $s_{\mathrm{in}}=\sqrt{\varepsilon_{\mathrm{in}}} / \sqrt{\varepsilon_{\mathrm{m}}}$, and the size parameter $x=k_{\mathrm{m}} a$. These solve the problem for Mie theory and now we can make use of these susceptibilities to calculate scattering quantities such as: scattering, absorption, and extinction cross-sections for the problems of our interest.

\section{Scattering Quantities}

In scattering problems, the EM characteristics that are most often considered are the absorption, scattering, and extinction cross-sections or coefficients. For the basic concepts of the general scattering theory, we can define the following quantities:

\section{Definitions}

Scattered power: $P_{\text {Sca }}[\mathrm{W}]$ is the power radiated by the scattered electromagnetic field $\left(\mathbf{E}_{\text {Sca }}\right)$.

Absorbed power: $P_{\text {Abs }}[\mathrm{W}]$ is the total power absorbed in the dielectric/metallic objects during the scattering process.

Extinguished power: $P_{\text {Ext }}=P_{\text {sca }}+P_{\text {abs }}[\mathrm{W}]$ corresponds to the power extracted from the incident plane wave.

The above quantities can be calculated using the following expression [38]:

$$
\begin{aligned}
& P_{\mathrm{Sca}}=\frac{1}{2} \mathcal{R}\left(\iint_{s}\left[\mathbf{E}_{\mathrm{Sca}} \times \mathbf{H}_{\mathrm{Sca}}^{*}\right] \cdot \hat{\mathbf{n}} r^{2} d S\right), \\
& P_{\mathrm{Abs}}=-\frac{1}{2} \mathcal{R}\left(\iint_{s}\left[\mathbf{E}_{\mathrm{out}} \times \mathbf{H}_{\mathrm{out}}^{*}\right] \cdot \hat{\mathbf{n}} r^{2} d S\right),
\end{aligned}
$$


where $S$ is a surface of a imaginary sphere enclosing all dielectric/metallic objects, $\hat{\mathbf{n}}$ is the unit vector normal to the surface (pointing outwards), and $\mathbf{E}_{\text {out }}=\mathbf{E}_{\text {Inc }}+\mathbf{E}_{\text {Sca }}$. So, we can show that,

$$
P_{\mathrm{Ext}}=-\frac{1}{2} \mathcal{R}\left(\iint_{s}\left[\mathbf{E}_{\mathrm{Inc}} \times \mathbf{H}_{\mathrm{Sca}}^{*}+\mathbf{E}_{\mathrm{Sca}} \times \mathbf{H}_{\mathrm{Inc}}^{*}\right] \cdot \hat{\mathbf{n}} r^{2} d S\right) .
$$

For us to calculate the integral over the surface, we need to use some important integrals shown in Appendix C, and the Wronskian identity of the Riccatti's spherical Bessel functions $\chi_{n} \psi_{n}^{\prime}-\chi_{n}^{\prime} \psi_{n}=1$. The results give:

$$
\begin{aligned}
P_{\text {Sca }} & =\frac{1}{2} \mathcal{R}\left(\iint_{s}\left[\mathbf{E}_{\mathrm{sca}} \times \mathbf{H}_{\mathrm{sca}}^{*}\right] \cdot \hat{\mathbf{n}} r^{2} d S\right), \\
& =\frac{1}{2} \frac{\left|E_{0}\right|^{2}}{\omega \mu k_{\mathrm{m}}} \sum_{n, m}\left[\left|p_{n m}\right|^{2}+\left|q_{n m}\right|^{2}\right] .
\end{aligned}
$$

A similar approach can be applied for the extinguished power and yields:

$$
P_{\text {Ext }}=-\frac{1}{2} \frac{\left|E_{0}\right|^{2}}{\omega \mu k_{\mathrm{m}}} \sum_{n, m}\left[\mathcal{R}\left(a_{n m} p_{n m}^{*}\right)+\mathcal{R}\left(b_{n m} q_{n m}^{*}\right)\right],
$$

where $\mathcal{R}$ denotes the real part. The cross-section $(\sigma)\left[\mathrm{m}^{2}\right]$ is then defined w.r.t the incident field intensity (power density) $S_{\text {Inc }}=\varepsilon_{0} c \sqrt{\varepsilon_{\mathrm{m}}}\left|E_{0}\right|^{2} / 2\left[\mathrm{~W} / \mathrm{m}^{2}\right]$ as,

$$
\sigma_{i}=\frac{P_{i}}{S_{\text {Inc }}}
$$

This leads to:

$$
\begin{aligned}
\sigma_{\text {Sca }} & =\frac{1}{k_{\mathrm{m}}^{2}} \sum_{n, m}\left[\left|a_{n m}\right|^{2}\left|\Gamma_{n}\right|^{2}+\left|b_{n m}\right|^{2}\left|\Delta_{n}\right|^{2}\right] \\
\sigma_{\mathrm{Ext}} & =-\frac{1}{k_{\mathrm{m}}^{2}} \sum_{n, m}\left[\left|a_{n m}\right|^{2} \mathcal{R}\left(\Gamma_{n}\right)+\left|b_{n m}\right|^{2} \mathcal{R}\left(\Delta_{n}\right)\right] \\
\sigma_{\mathrm{Abs}} & =\sigma_{\mathrm{Ext}}-\sigma_{\mathrm{Sca}} .
\end{aligned}
$$

Therefore to use the solutions of Mie theory, we need to know the size parameter, the coefficients of the incident field $\left(a_{n m}\right.$ and $\left.b_{n m}\right)$, and the dielectric functions of all media.

\section{Plane Wave Excitation}

For plane wave excitation of a sphere, if we consider a plane wave propagating along $\hat{\mathbf{e}}_{z}$ and polarised along $\hat{\mathbf{e}}_{x}$, the complex incident field is then:

$$
\mathbf{E}_{\text {Inc }}(\mathbf{r})=\exp \left\{i k_{\mathrm{m}} z\right\} E_{\mathrm{Inc}} \hat{\mathbf{e}}_{x}
$$


where $E_{\text {Inc }}$ denotes its amplitude. Therefore, it is natural to take $E_{0}=E_{\text {Inc }}$ as a reference amplitude for the expansion. The power of the incident wave is then $P_{\mathrm{Inc}}=$ $\left|S_{0}\right|=\left|E_{0} H_{0}\right| / 2=\varepsilon_{0} c \sqrt{\varepsilon_{\mathrm{m}}}\left|E_{0}\right|^{2} / 2$. The expansion of this plane wave excitation field in VSHs is given by Eq. 2.22, where the coefficients $a_{n m}$ and $b_{n m}$ can be found (in Refs. [27, 38]) as:

$$
\begin{aligned}
a_{n m} & =b_{n m}=0, \quad \forall|m| \neq 1 \\
a_{n, m=1} & =i^{n+1} \sqrt{\pi(2 n+1)} \\
b_{n, m=1} & =i^{n+1} \sqrt{\pi(2 n+1)} \\
a_{n, m=-1} & =i^{n+1} \sqrt{\pi(2 n+1)} \\
b_{n, m=-1} & =-i^{n+1} \sqrt{\pi(2 n+1)} .
\end{aligned}
$$

With these coefficients, Eqs. 2.41 and 2.42 will become:

$$
\begin{aligned}
\sigma_{\text {Sca }} & =\frac{\pi}{k_{\mathrm{m}}^{2}} \sum_{n=1}^{\infty}(2 n+1)\left[\left|\Gamma_{n}\right|^{2}+\left|\Delta_{n}\right|^{2}\right] \\
\sigma_{\mathrm{Ext}} & =-\frac{\pi}{k_{\mathrm{m}}^{2}} \sum_{n=1}^{\infty}(2 n+1)\left[\Re\left(\Gamma_{n}\right)+\Re\left(\Delta_{n}\right)\right] .
\end{aligned}
$$

And the absorption cross-section is simply $\sigma_{\mathrm{Abs}}=\sigma_{\mathrm{Ext}}-\sigma_{\mathrm{Sca}}$.

\subsubsection{Scattering by a Coated Sphere (Core-Shell System)}

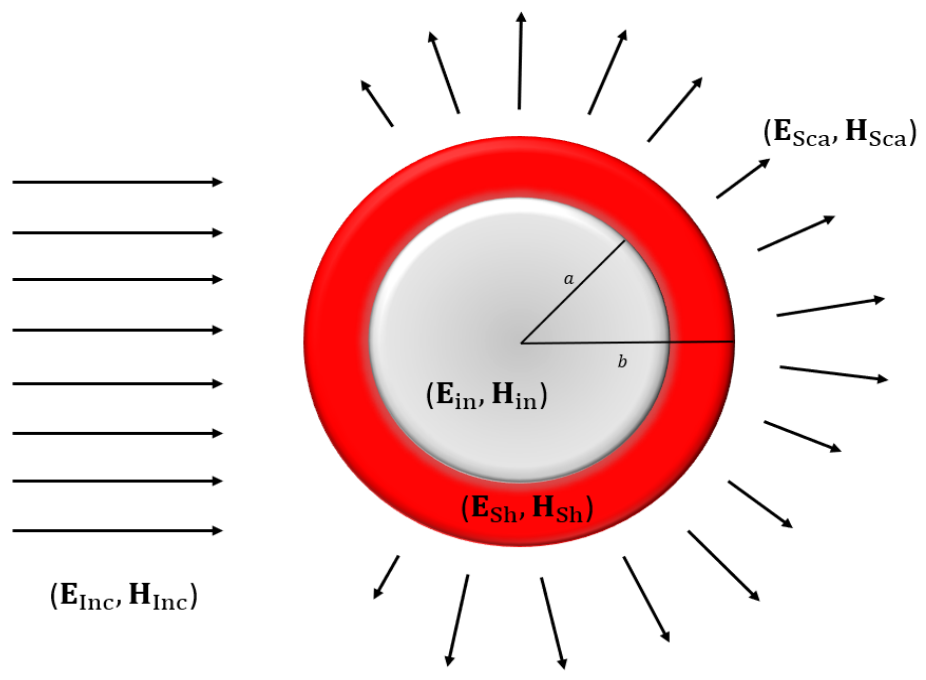

Figure 2.1: The incident field $\left(\mathbf{E}_{\text {Inc }}, \mathbf{H}_{\text {Inc }}\right)$ gives rise to the fields $\left(\mathbf{E}_{\text {in }}, \mathbf{H}_{\text {in }}\right)$ and $\left(\mathbf{E}_{\mathrm{Sh}}, \mathbf{H}_{\mathrm{Sh}}\right)$ inside the sphere and shell, respectively, and a scattered field $\left(\mathbf{E}_{\mathrm{Sca}}, \mathbf{H}_{\mathrm{Sca}}\right)$ in the medium surrounding the coated particle. 
Core-shell spherical nanostructures have been produced from a wide variety of materials, and the electromagnetic interaction between light and such multilayered structures can lead to many interesting functionalities, notably through the excitation of surface waves $[28,53,54]$. The core-shell structure has been used in many recent works with dye-coated core-shell nanostructures, which have become a useful platform to study the interaction between plasmon and molecular resonances in either weak or strong coupling regimes $[25,34,55,56]$.

We here consider a sphere of radius $a$ coated or surrounded by a spherical shell of thickness $b-a$ (outer radius $b$, and inner radius $a$, as depicted in Fig. 2.1). Because the volume of the shell does not contain the origin or infinity, the most general expansion is in terms of both $\left(\mathbf{M}^{(1)}, \mathbf{N}^{(1)}\right)$ and $\left(\mathbf{M}^{(3)}, \mathbf{N}^{(3)}\right)$. This is equivalent to the expansion in terms of $\left(\mathbf{M}^{(1)}, \mathbf{N}^{(1)}\right)$ and $\left(\mathbf{M}^{(2)}, \mathbf{N}^{(2)}\right)$ as third kind VSHs are the linear combinations of the first and second kinds of VSHs. Therefore, the solutions to the vector Helmholtz equation for a spherical shell can then take the form of the first and second kinds of the Bessel functions. With $\varepsilon_{\mathrm{s}}$ and $k_{\mathrm{s}}$ being the dielectric constant and wave-vector (respectively) for the spherical shell, the fields inside it can be defined as:

$$
\begin{aligned}
& \mathbf{E}_{\mathrm{Sh}}=E_{0} \sum_{n, m} e_{n m} \mathbf{M}_{n m}^{(1)}\left(k_{\mathrm{s}}, \mathbf{r}\right)+f_{n m} \mathbf{N}_{n m}^{(1)}\left(k_{\mathrm{s}}, \mathbf{r}\right)+g_{n m} \mathbf{M}_{n m}^{(2)}\left(k_{\mathrm{s}}, \mathbf{r}\right)+h_{n m} \mathbf{N}_{n m}^{(2)}\left(k_{\mathrm{s}}, \mathbf{r}\right), \\
& \mathbf{H}_{\mathrm{Sh}}=\frac{k_{\mathrm{s}}}{i \omega \mu} E_{0} \sum_{n, m} e_{n m} \mathbf{N}_{n m}^{(1)}\left(k_{\mathrm{s}}, \mathbf{r}\right)+f_{n m} \mathbf{M}_{n m}^{(1)}\left(k_{\mathrm{s}}, \mathbf{r}\right)+g_{n m} \mathbf{N}_{n m}^{(2)}\left(k_{\mathrm{s}}, \mathbf{r}\right)+h_{n m} \mathbf{M}_{n m}^{(2)}\left(k_{\mathrm{s}}, \mathbf{r}\right) .
\end{aligned}
$$

The boundary conditions for this case are different from a single sphere problem. We now have boundary conditions on the particle's (or sphere's) surface at $r=a$, and on the spherical shell's surface at $r=b$. At $r=a$, we have:

$$
\begin{aligned}
& \mathbf{E}_{\mathrm{Sh}} \cdot \hat{\theta}=\mathbf{E}_{\mathrm{in}} \cdot \hat{\theta} \\
& \mathbf{E}_{\mathrm{Sh}} \cdot \hat{\phi}=\mathbf{E}_{\mathrm{in}} \cdot \hat{\phi} \\
& \mathbf{H}_{\mathrm{Sh}} \cdot \hat{\theta}=\mathbf{H}_{\text {in }} \cdot \hat{\theta} \\
& \mathbf{H}_{\mathrm{Sh}} \cdot \hat{\phi}=\mathbf{H}_{\text {in }} \cdot \hat{\phi} .
\end{aligned}
$$


And at $r=b$, we have:

$$
\begin{aligned}
& \mathbf{E}_{\text {out }} \cdot \hat{\theta}=\mathbf{E}_{\mathrm{Sh}} \cdot \hat{\theta} \\
& \mathbf{E}_{\mathrm{out}} \cdot \hat{\phi}=\mathbf{E}_{\mathrm{Sh}} \cdot \hat{\phi} \\
& \mathbf{H}_{\mathrm{out}} \cdot \hat{\theta}=\mathbf{H}_{\mathrm{Sh}} \cdot \hat{\theta} \\
& \mathbf{H}_{\mathrm{out}} \cdot \hat{\phi}=\mathbf{H}_{\mathrm{Sh}} \cdot \hat{\phi} .
\end{aligned}
$$

With these boundary conditions, the susceptibilities of the scattered field $\left(\Gamma_{n}\right.$ and $\Delta_{n}$ ) can be determined as follows. We first apply the boundary conditions at $r=a$, and get:

$$
\begin{aligned}
{\left[e_{n m} \psi_{n}\left(s_{\mathrm{s}} y\right)+g_{n m} \chi_{n}\left(s_{\mathrm{s}} y\right)\right] / s_{\mathrm{s}} } & =c_{n m} \psi_{n}\left(s_{\mathrm{in}} y\right) / s_{\text {in }} \\
{\left[f_{n m} \psi_{n}^{\prime}\left(s_{\mathrm{s}} y\right)+h_{n m} \chi_{n}^{\prime}\left(s_{\mathrm{s}} y\right)\right] / s_{\mathrm{s}} } & =d_{n m} \psi_{n}^{\prime}\left(s_{\mathrm{in}} y\right) / s_{\text {in }} \\
e_{n m} \psi_{n}^{\prime}\left(s_{\mathrm{s}} y\right)+g_{n m} \chi_{n}^{\prime}\left(s_{\mathrm{s}} y\right) & =c_{n m} \psi_{n}^{\prime}\left(s_{\mathrm{in}} y\right) \\
f_{n m} \psi_{n}\left(s_{\mathrm{s}} y\right)+h_{n m} \chi_{n}\left(s_{\mathrm{s}} y\right) & =d_{n m} \psi_{n}\left(s_{\mathrm{in}} y\right),
\end{aligned}
$$

where $s_{\mathrm{s}}=\sqrt{\varepsilon_{\mathrm{s}}} / \sqrt{\varepsilon_{\mathrm{m}}}, s_{\mathrm{in}}=\sqrt{\varepsilon_{\mathrm{in}}} / \sqrt{\varepsilon_{\mathrm{m}}}$, and $y=k_{\mathrm{m}} a$. These equations then lead to:

$$
\begin{aligned}
& \frac{g_{n m}}{e_{n m}}=-\frac{s_{\mathrm{s}} \psi_{n}^{\prime}\left(s_{\mathrm{s}} y\right) \psi_{n}\left(s_{\mathrm{in}} y\right)-s_{\text {in }} \psi_{n}\left(s_{\mathrm{s}} y\right) \psi_{n}^{\prime}\left(s_{\mathrm{in}} y\right)}{s_{\mathrm{s}} \chi_{n}^{\prime}\left(s_{\mathrm{s}} y\right) \psi_{n}\left(s_{\mathrm{in}} y\right)-s_{\text {in }} \chi_{n}\left(s_{\mathrm{s}} y\right) \psi_{n}^{\prime}\left(s_{\mathrm{in}} y\right)}=\Gamma_{n}^{(1)} \\
& \frac{h_{n m}}{f_{n m}}=-\frac{s_{\mathrm{s}} \psi_{n}\left(s_{\mathrm{s}} y\right) \psi_{n}^{\prime}\left(s_{\mathrm{in}} y\right)-s_{\text {in }} \psi_{n}^{\prime}\left(s_{\mathrm{s}} y\right) \psi_{n}\left(s_{\mathrm{in}} y\right)}{s_{\mathrm{s}} \chi_{n}\left(s_{\mathrm{s}} y\right) \psi_{n}^{\prime}\left(s_{\mathrm{in}} y\right)-s_{\text {in }} \chi_{n}^{\prime}\left(s_{\mathrm{s}} y\right) \psi_{n}\left(s_{\mathrm{in}} y\right)}=\Delta_{n}^{(1)} .
\end{aligned}
$$

The boundary conditions at $r=b$ gives:

$$
\begin{aligned}
a_{n m} \psi_{n}(x)+p_{n m} \xi_{n}(x) & =\left[e_{n m} \psi_{n}\left(s_{\mathrm{s}} x\right)+g_{n m} \chi_{n}\left(s_{\mathrm{s}} x\right)\right] / s_{\mathrm{t}} \\
b_{n m} \psi_{n}^{\prime}(x)+q_{n m} \xi_{n}^{\prime}(x) & =\left[f_{n m} \psi_{n}^{\prime}\left(s_{\mathrm{s}} x\right)+h_{n m} \chi_{n}^{\prime}\left(s_{\mathrm{s}} x\right)\right] / s_{\mathrm{s}} \\
a_{n m} \psi_{n}^{\prime}(x)+p_{n m} \xi_{n}^{\prime}(x) & =e_{n m} \psi_{n}^{\prime}\left(s_{\mathrm{s}} x\right)+g_{n m} \chi_{n}^{\prime}\left(s_{\mathrm{s}} x\right) \\
b_{n m} \psi_{n}(x)+q_{n m} \xi_{n}(x) & =f_{n m} \psi_{n}\left(s_{\mathrm{s}} x\right)+h_{n m} \chi_{n}\left(s_{\mathrm{s}} x\right),
\end{aligned}
$$

where $x=k_{\mathrm{m}} b$. These equations together with Eqs. 2.58 and 2.59 yield:

$$
\begin{aligned}
\Gamma_{n} & =-\frac{[\psi \psi]_{n, x}^{m, s}[\chi \psi]_{n, y}^{m, c}-[\chi \psi]_{n, x}^{m, s}[\psi \psi]_{n, y}^{m, c}}{[\psi \xi]_{n, x}^{m, s}[\chi \psi]_{n, y}^{m, c}-[\chi \xi]_{n, x}^{m, s}[\psi \psi]_{n, y}^{m, c}} \\
\Delta_{n} & =-\frac{[\psi \psi]_{n, x}^{e, s}[\chi \psi]_{n, c}^{e, c}-[\chi \psi]_{n, x}^{e, s}[\psi \psi]_{n, y}^{e, c}}{[\psi \xi]_{n, x}^{e, s}[\chi \psi]_{n, y}^{e, c}-[\chi \xi]_{n, x}^{e, s}[\psi \psi]_{n, y}^{e, c}}
\end{aligned}
$$

where

$$
\begin{aligned}
& {[\Lambda \psi]_{n, y}^{m, c}=s_{\text {in }} \Lambda_{n}\left(s_{\mathrm{s}} y\right) \psi_{n}^{\prime}\left(s_{\mathrm{in}} y\right)-s_{\mathrm{s}} \Lambda_{n}^{\prime}\left(s_{\mathrm{s}} y\right) \psi_{n}\left(s_{\mathrm{in}} y\right),} \\
& {[\Lambda \psi]_{n, y}^{e, c}=s_{\mathrm{in}} \Lambda_{n}^{\prime}\left(s_{\mathrm{s}} y\right) \psi_{n}\left(s_{\mathrm{in}} y\right)-s_{\mathrm{s}} \Lambda_{n}\left(s_{\mathrm{s}} y\right) \psi_{n}^{\prime}\left(s_{\mathrm{in}} y\right),} \\
& {[\Lambda \Omega]_{n, s}^{m, s}=s_{\mathrm{s}} \Lambda_{n}^{\prime}\left(s_{\mathrm{s}} x\right) \Omega_{n}(x)-\Lambda_{n}\left(s_{\mathrm{s}} x\right) \Omega_{n}^{\prime}(x),} \\
& {[\Lambda \Omega]_{n, x}^{e, s}=s_{\mathrm{s}} \Lambda_{n}\left(s_{\mathrm{s}} x\right) \Omega_{n}^{\prime}(x)-\Lambda_{n}^{\prime}\left(s_{\mathrm{s}} x\right) \Omega_{n}(x),} \\
& \Lambda=\psi \text { or } \chi, \text { and } \Omega=\psi \text { or } \xi .
\end{aligned}
$$


With these new susceptibilities, we will be able to calculate the extinction, scattering, and absorption cross-sections of the coated particle system.

\subsection{Coupled Dipole Theory or Model (CDM)}

In this section, we consider the light scattering by particles or molecules with dimensions much smaller than the wavelength so that the dipole approximation can be used to model each molecule as a point dipole. We are interested in a scattering medium containing a reasonably large number of molecules (approximately a few thousands) in close enough distance such that the coupling between their near-neighbour cannot be neglected, but sufficiently far apart that the excitation of higher-order multipoles can be safely ignored.

We here consider a collection of $N$ point electric dipoles $\mathbf{p}_{i}$ located at $\mathbf{r}_{i}(i=$ $1 \ldots N)$ and embedded in a homogeneous, non-absorbing, isotropic, non-magnetic infinite medium, which characterised by a real refractive index $n_{\mathrm{m}}$ (or dielectric constant $\varepsilon_{\mathrm{m}}$ ). The dipoles are subject to an incident electric field $\mathbf{E}_{\text {Inc }}$. For point dipoles described by a polarisability tensor $\underline{\alpha}_{\mu}$, the dipole moment induced by the incident electric field is given by:

$$
\mathbf{p}_{i}=\underline{\alpha}_{\mu} \mathbf{e}_{i}
$$

where $\mathbf{e}_{i}$ denotes the microscopic field at the dipole position. At this stage, we need to know the link between the microscopic field (the field that is felt by a molecule embedded in a medium of dielectric constant $\varepsilon_{\mathrm{m}}$ ) and the macroscopic field described by the Maxwell equations for media. The relationship between the two is given by $[27]:$

$$
\mathbf{e}_{i}=\frac{\varepsilon_{\mathrm{m}}+2}{3} \mathbf{E}_{i}=L_{\mathrm{m}} \mathbf{E}_{i}
$$

where $L_{\mathrm{m}}$ refers to the local field correction, which represents the difference between macroscopic and microscopic fields. This correction is important in the framework of the optical properties of dielectric media (i.e, molecules embedded in a non-vacuum medium, for example), as explained in Appendix C of Ref. [27]. This local field correction will also be useful for determining the dielectric function(s) for molecular monolayer in non-vacuum media, presented in Chapter 4.

At a microscopic level, a dipole responds to the applied field with an intrinsic polarisability $\underline{\alpha}_{\mu}$. However, for relevant experiments, they are mostly done in solution. 
Therefore, it is important to include this local field correction. From a reciprocity argument, such a dipole moment will produce a field, which is also enhanced by the same local field correction factor. Therefore, we may find the effective macroscopic polarisability $\underline{\alpha}_{i}$ as:

$$
\underline{\alpha}_{i}=L_{\mathrm{m}}^{2} \underline{\alpha}_{\mu}
$$

The macroscopic electric field created by the dipole $\mathbf{p}_{i}$ at a point $\mathbf{r}$ also depends linearly on that dipole moment itself, which is:

$$
\mathbf{E}_{i}(\mathbf{r})=\mathbb{G}\left(\mathbf{r}_{i}, \mathbf{r}\right) \mathbf{p}_{i}
$$

where $\mathbb{G}$ is Green's tensor which characterises the electric field at $\mathbf{r}$ created by a point dipole $\mathbf{p}_{i}$ at $\mathbf{r}_{i}$.

\subsubsection{Green's Tensor}

The wave equation with a point source (corresponding to an oscillating dipole) will be used to define the relationship between the dipole moment and its corresponding macroscopic electric field. For an oscillating dipole in a medium with refractive index $n_{\mathrm{m}}$, the wave equation for the vector potential $\mathbf{A}$ (with Lorentz gauge) can be defined as:

$$
\nabla^{2} \mathbf{A}+k_{\mathrm{m}}^{2} \mathbf{A}=\mu_{0} \mathbf{j}
$$

where $k_{\mathrm{m}}=n_{\mathrm{m}} k_{0}$ is the wave-vector in the medium, and $k_{0}=\omega / c$ in a vacuum (with $\omega$ is the frequency and $c$ is the speed of light). The vector $\mathbf{j}$ is the macroscopic source term corresponding to the oscillating dipole at a frequency $\omega$, which is:

$$
\mathbf{j}(\mathbf{r})=-i \omega \mathbf{p} \delta(\mathbf{r})
$$

Solving for A leads to the standard Green's function for the Helmholtz equation, which is:

$$
\mathbf{A}=-i \omega \mu_{0} \frac{\mathbf{p} e^{i k_{\mathrm{m}} r}}{4 \pi r}
$$

We then can use this to deduce the magnetic field as $\mathbf{H}=(\nabla \times \mathbf{A}) / \mu_{0}$, which leads to:

$$
\mathbf{H}=\frac{\omega k_{\mathrm{m}}}{4 \pi r} e^{i k_{\mathrm{m}} r}\left(1-\frac{1}{i k_{\mathrm{m}} r}\right) \hat{\mathbf{r}} \times \mathbf{p}
$$


According to Maxwell's equation, the electric field can be found as $\mathbf{E}=i(\nabla \times$ $\mathbf{H}) /\left(\omega \varepsilon_{0} \varepsilon_{\mathrm{m}}\right)$. Therefore, the macroscopic electric field of an oscillating dipole in a medium can be expressed as:

$$
\mathbf{E}(\mathbf{r})=\frac{1}{4 \pi \varepsilon_{0} \varepsilon_{\mathrm{m}}} \frac{e^{i k_{\mathrm{m}} r}}{r}\left[k_{\mathrm{m}}^{2}[\mathbf{p}-(\hat{\mathbf{r}} \cdot \mathbf{p}) \hat{\mathbf{r}}]+\left(\frac{1}{r^{2}}-\frac{i k_{\mathrm{m}}}{r}\right)[3 \hat{\mathbf{r}}(\hat{\mathbf{r}} \cdot \mathbf{p})-\mathbf{p}]\right],
$$

We can rewrite this expression as $\mathbf{E}_{i}(\mathbf{r})=\mathbb{G}\left(\mathbf{r}_{i}, \mathbf{r}\right) \mathbf{p}_{i}$ (as done in Eq. 2.66) for a general dipole $\mathbf{p}_{i}$ located at $\mathbf{r}_{i}$. By defining $\mathbf{R}=\mathbf{r}-\mathbf{r}_{i}, R=|\mathbf{R}|$, and $\hat{\mathbf{R}}=\mathbf{R} / R$, the Green's tensor can then be found as:

$$
\mathbb{G}\left(\mathbf{r}_{i}, \mathbf{r}\right)=\frac{1}{4 \pi \varepsilon_{0} \varepsilon_{\mathrm{m}}} \frac{e^{i k_{\mathrm{m}} R}}{R}\left[k_{\mathrm{m}}^{2}(\mathbb{I}-\hat{\mathbf{R}} \otimes \hat{\mathbf{R}})+\left(\frac{1}{R^{2}}-\frac{i k_{\mathrm{m}}}{R}\right)(3 \hat{\mathbf{R}} \otimes \hat{\mathbf{R}}-\mathbb{I})\right]
$$

\subsubsection{Coupled Dipole Equations}

We now consider the case of a collection $N$ of polarisable point dipoles, excited by an incident field. The local field at a dipole's location is the net macroscopic field that is the sum of the incident field and the field scattered (radiated) by its neighbours. Hence, the dipole moment $\mathbf{p}_{i}$ located at $\mathbf{r}_{i}$ will take the form of:

$$
\begin{aligned}
\mathbf{p}_{i}=\underline{\alpha}_{i} \mathbf{E}_{i} & =\underline{\alpha}_{i}\left(\mathbf{E}_{\mathrm{Inc}}\left(\mathbf{r}_{i}\right)+\sum_{j \neq i} \mathbb{G}_{i j} \mathbf{p}_{j}\right) \\
& =\underline{\alpha}_{i}\left(\mathbf{E}_{\text {Inc }}\left(\mathbf{r}_{i}\right)+\sum_{j \neq i} \mathbb{G}_{i j} \underline{\alpha}_{j} \mathbf{E}_{j}\right) \\
\text { i.e. } \quad \mathbf{E}_{i} & =\mathbf{E}_{\text {Inc }}\left(\mathbf{r}_{i}\right)+\sum_{j \neq i} \mathbb{G}_{i j} \underline{\alpha}_{j} \mathbf{E}_{j},
\end{aligned}
$$

where $\mathbf{E}_{\text {Inc }}\left(\mathbf{r}_{i}\right)$ is the incident field, and $\mathbb{G}_{i j}=\mathbb{G}\left(\mathbf{r}_{i}, \mathbf{r}_{j}\right)$ is the Green's tensor for the field created by dipole $j$ at the location of dipole $i$, which is:

$$
\mathbb{G}_{i j}=\frac{1}{4 \pi \varepsilon_{0} \varepsilon_{\mathrm{m}}} \frac{e^{i k_{\mathrm{m}} r_{i j}}}{r_{i j}}\left[k_{\mathrm{m}}^{2}\left(\mathbb{I}-\hat{\mathbf{r}}_{i j} \otimes \hat{\mathbf{r}}_{i j}\right)+\left(\frac{1}{r_{i j}^{2}}-\frac{i k_{\mathrm{m}}}{r_{i j}}\right)\left(3 \hat{\mathbf{r}}_{i j} \otimes \hat{\mathbf{r}}_{i j}-\mathbb{I}\right)\right] .
$$

In a matrix form, the following linear system can be considered:

$$
\mathbb{L}_{\underline{\mathbf{E}}_{i}}=\underline{\mathbf{E}}_{\mathrm{Inc}} .
$$

$\mathbb{L}$ represents the interaction matrix, whose diagonal blocks consists of $3 \times 3$ identity matrices $\mathbb{I}$, and each of its off-diagonal blocks is of the form $\mathbb{L}_{i j}=-\mathbb{G}_{i j} \underline{\alpha}_{j}$. This is 
a linear system of $3 N$ equations in the matrix form with vectors $\underline{\mathbf{E}}_{i}, \underline{\mathbf{p}}_{i}$, and $\underline{\mathbf{E}}_{\text {Inc }}$ of length $3 N$, where $N$ is the number of dipoles. To solve for the self-consistent macroscopic electric field $\mathbf{E}$ at each dipole location, the inverse of $\mathbb{L}$ (a $3 N \times 3 N$ matrix) is therefore needed. This linear system of equations can be solved by standard linear algebra routines. However, the required time to solve for the solutions will depend on the number of dipoles. For example, if the number of dipoles is increased by $n$ (i.e. number of dipoles $=n N)$ then the size system of the matrix $\mathbb{L}$ will be $(3 n N \times 3 n N)$. Hence, the simulation time will increase by $n^{2}$ compared to the system of $N$-dipole.

\subsubsection{Scattering, Absorption, and Extinction}

As the electric field and the dipole moment at each dipole's position can be computed, we can use them to determine the scattering, absorption, and extinction cross-sections of the system.

\section{A single dipole}

By following the standard electromagnetic theory, which includes the microscopic local field corrections $\left(L_{\mathrm{m}}^{2}\right.$ is no longer hidden in $\alpha$, i.e. $\alpha=\alpha_{\mu}$ ), the scattering power for a single isolated dipole in a medium can be derived as (Ref. [27], Eq. 2.46):

$$
P_{\text {Sca }}=\frac{\omega^{4} n_{\mathrm{m}} L_{\mathrm{m}}^{2}}{12 \pi \varepsilon_{0} c^{3}}\left|\mathbf{p}_{i}\right|^{2} .
$$

And the absorption power can be expressed [Ref. [27], Eqs. (4.77 and 4.79)] as:

$$
P_{\mathrm{Abs}}=\frac{\omega L_{\mathrm{m}}^{2}}{2} \Im\left\{\mathbf{p}_{i} \cdot \mathbf{E}^{*}\right\} .
$$

This absorption power does include the radiative correction term due to the selfradiated field of the dipole itself in the total electric field at the dipole position $\mathbf{E}$. We can then re-express Eq. 2.78 as:

$$
\begin{aligned}
P_{\text {Abs }} & =\frac{\omega L_{\mathrm{m}}^{2}}{2} \Im\left\{\mathbf{p}_{i} \cdot \mathbf{E}_{i}^{*}\right\}-\frac{\omega^{4} n_{\mathrm{m}} L_{\mathrm{m}}^{2}}{12 \pi \varepsilon_{0} c^{3}}\left|\mathbf{p}_{i}\right|^{2} \\
& =\frac{\omega L_{\mathrm{m}}^{2}}{2} \Im\left\{\mathbf{p}_{i} \cdot \mathbf{E}_{i}^{*}\right\}-P_{\text {Sca }} .
\end{aligned}
$$

The extinction power can then be computed as $P_{\text {Ext }}=P_{\text {Sca }}+P_{\text {Abs }}$, which is:

$$
P_{\text {Ext }}=\frac{\omega L_{\mathrm{m}}^{2}}{2} \Im\left\{\mathbf{p}_{i} \cdot \mathbf{E}_{i}^{*}\right\}
$$

In principle, the effect from the radiative correction for molecules is typically negligible (because the scattering is very small). For absorbing molecules, where $\Im\{\alpha\}>0$, the radiative correction will be insignificant. 


\section{Collection of dipoles}

The expressions described in the previous section can be generalised to a collection of polarisable dipoles excited by an incident electric field $\mathbf{E}_{\mathrm{Inc}}$. The net macroscopic field $\mathbf{E}_{i}$ corresponding to the local electric field at the location of dipole $i$ (excluding the self-reacted field), is given by the solution of Eq. 2.74. The total absorption power for all the dipoles can be calculated by summing all the absorption of each individual dipole, which leads to:

$$
P_{\mathrm{Abs}}=\sum_{i}\left(\frac{\omega L_{\mathrm{m}}^{2}}{2} \Im\left\{\mathbf{p}_{i} \cdot \mathbf{E}_{i}^{*}\right\}-\frac{\omega^{4} n_{\mathrm{m}} L_{\mathrm{m}}^{2}}{12 \pi \varepsilon_{0} c^{3}}\left|\mathbf{p}_{i}\right|^{2}\right)
$$

where the second term accounts for the self-reaction. The power extinguished from the incident field (extinction power) can be obtained by generalising Eq. 2.81:

$$
P_{\text {Ext }}=\sum_{i} \frac{\omega L_{\mathrm{m}}^{2}}{2} \Im\left\{\mathbf{p}_{i} \cdot \mathbf{E}_{i}^{*}\right\} .
$$

For the scattering power, it may be computed as $P_{\text {Sca }}=P_{\text {Ext }}-P_{\text {Abs }}$. It can also be calculated by integrating the flux of the Poynting vector over all directions. We may numerically integrate the flux of time-averaged Poynting vector $\langle\mathbf{S}\rangle=\Re\left\{\mathbf{E}_{i} \times \mathbf{H}_{i}^{*}\right\} / 2$ over a sphere of radius $a$, chosen arbitrarily far away from the origin for convenience, so that only the far-field components need to be considered. This gives,

$$
\begin{aligned}
P_{\text {Sca }} & =\frac{1}{2} \Re\left(\iint_{S}\left[\mathbf{E}_{i} \times \mathbf{H}_{i}^{*}\right] \cdot \hat{\mathbf{n}} d S\right) \\
& =\frac{L_{\mathrm{m}}^{2}}{2 Z_{1}} \iint_{S}\left|\mathbf{E}_{i}\right|^{2} d S,
\end{aligned}
$$

where $Z_{1}=\sqrt{\mu_{0} /\left(\varepsilon_{\mathrm{m}} \varepsilon_{0}\right)}$ is the wave impedance in the incident medium. With Eq. 2.71, we can rewrite Eq. 2.85 as:

$$
\begin{aligned}
P_{\text {Sca }} & \left.=\left|\frac{k_{\mathrm{m}}^{2}}{4 \pi \varepsilon_{0} \varepsilon_{\mathrm{m}}} \frac{e^{i k_{\mathrm{m}} a}}{a}\right|^{2} \frac{L_{\mathrm{m}}^{2}}{2 Z_{1}} \iint_{S} \mid \sum_{i}\left(\mathbf{p}_{i}-\mathbf{p}_{i} \cdot \hat{\mathbf{n}}\right) \hat{\mathbf{n}}\right)\left.e^{-i k_{\mathrm{m}} \mathbf{r}_{i} \cdot \hat{\mathbf{n}}}\right|^{2} d S \\
& =\frac{k_{\mathrm{m}}^{4}}{\left(4 \pi \varepsilon_{0} \varepsilon_{\mathrm{m}}\right)^{2}} \frac{L_{\mathrm{m}}^{2}}{8 \pi Z_{1}} \iint_{\Omega}\left|\sum_{i}(\mathbb{I}-\hat{\mathbf{n}} \otimes \hat{\mathbf{n}}) \mathbf{p}_{i} e^{-i k_{\mathrm{m}} \mathbf{r}_{i} \cdot \hat{\mathbf{n}}}\right|^{2} d \Omega,
\end{aligned}
$$

where $\Omega$ is the full solid angle. The conservation of energy $P_{\mathrm{Sca}}=P_{\mathrm{Ext}}-P_{\mathrm{Abs}}$ can be used as consistency check.

In the case of a plane wave excitation, we may write the incident field as, $\mathbf{E}_{\mathrm{Inc}}(\mathbf{r})=\mathbf{E}_{0} e^{i k_{\mathrm{m}} \hat{\mathbf{n}} \cdot \mathbf{r}}$. The power density of this incident electric field is then found 
as $S_{\text {Inc }}=\left|E_{0}\right|^{2} /\left(2 Z_{1}\right)$. From Eq. 2.40, the absorption, extinction, and scattering cross-sections will be defined as:

$$
\begin{aligned}
\sigma_{\mathrm{Abs}} & =\frac{2 \pi}{\lambda \varepsilon_{0} \sqrt{\varepsilon_{\mathrm{m}}}} \frac{L_{\mathrm{m}}^{2}}{\left|E_{0}\right|^{2}} \sum_{i} \Im\left(\left\{\mathbf{p}_{i} \cdot \mathbf{E}_{i}^{*}\right\}-\frac{k_{\mathrm{m}}^{3}}{6 \pi \varepsilon_{0} \varepsilon_{\mathrm{m}}}\left|\mathbf{p}_{i}\right|^{2}\right) \\
\sigma_{\mathrm{Ext}} & =\frac{2 \pi}{\lambda \varepsilon_{0} \sqrt{\varepsilon_{\mathrm{m}}}} \frac{L_{\mathrm{m}}^{2}}{\left|E_{0}\right|^{2}} \sum_{i} \Im\left\{\mathbf{p}_{i} \cdot \mathbf{E}_{i}^{*}\right\} \\
\sigma_{\mathrm{Sca}} & =\sigma_{\mathrm{Ext}}-\sigma_{\mathrm{Abs}} \quad \text { or } \\
& =\frac{k_{\mathrm{m}}^{4}}{\left(4 \pi \varepsilon_{0} \varepsilon_{\mathrm{m}}\right)^{2}} \frac{L_{\mathrm{m}}^{2}}{4 \pi\left|E_{0}\right|^{2}} \iint_{\Omega}\left|\sum_{i}(\mathbb{I}-\hat{\mathbf{n}} \otimes \hat{\mathbf{n}}) \mathbf{p}_{i} e^{-i k_{\mathrm{m}} \mathbf{r}_{i} \cdot \hat{\mathbf{n}}}\right|^{2} d \Omega .
\end{aligned}
$$

\subsection{Generalised Coupled-Dipole Model (GCDM)}

In this section, we will discuss briefly how the CDM in the previous section can be generalised to the case when the collection of $N$ dipoles is surrounding a spherical core (nanoparticle). More description of the theory can be referred to in the supplementary information of Ref. [37]. In the case where there is a spherical core, the electric field $\mathbf{E}_{i}$ defined in Eq. 2.74 will have extra components to it, which is:

$$
\mathbf{E}_{i}=\mathbf{E}_{\text {Inc }}\left(\mathbf{r}_{i}\right)+\mathbf{E}_{\text {Sphere }}\left(\mathbf{r}_{i}\right)+\sum_{j \neq i} \mathbb{G}_{i j} \underline{\alpha}_{j} \mathbf{E}_{j}+\sum_{\forall j} \mathbb{S}_{i j} \underline{\alpha}_{j} \mathbf{E}_{j}
$$

The term $\mathbf{E}_{\text {Sphere }}\left(\mathbf{r}_{i}\right)$ corresponds to the scattered field by the sphere (this can be computed by using Mie Theory), and the last term describes the dipole-dipole interactions mediated by the sphere. It is worthy to note that the term $\mathbb{S}_{i i}$ is non-zero, and it corresponds to the self-reflected field due to the presence of the sphere, or image-dipole effect. In a matrix form (similar to Eq. 2.76), it reads:

$$
\mathbb{L} \underline{\mathbf{E}}_{i}=\underline{\mathbf{E}}_{\mathrm{Inc}}+\underline{\mathbf{E}}_{\mathrm{Sphere}}
$$

where $\mathbb{L}$ is the full interaction matrix obtained by combining the $3 \times 3$ Green's tensors $\mathbb{G}_{i j}$ and $\mathbb{S}_{i j}$,

$$
\mathbb{L}= \begin{cases}\mathbb{I}_{3}-\mathbb{S}_{i i} \underline{\alpha}_{i}, & i=j \\ \mathbb{G}_{i j} \underline{\alpha}_{j}-\mathbb{S}_{i j} \underline{\alpha}_{j}, & i \neq j\end{cases}
$$

Again, we will need to numerically solve Eq. 2.93 to provide the self-consistent macroscopic field $\mathbf{E}_{i}$ for each dipole. Then the absorption, extinction, and scattering cross-sections can be calculated. More details of these can be found in Ref. [37]. 


\subsection{GCDM Vs. Mie Theory}

For the light scattering problem of molecules arranged in spherical shell, the preferred approach of the most recently refined model (Coupled Dipole Model, CDM) consists of a microscopic approach that treats each molecule as a polarisable dipole and calculate the self-consistent field created by mutual interaction of all the molecules. The solution is mathematically similar to the commonly used Discrete Dipole Approximation (DDA) [36]. This CDM was recently applied to molecular dipoles arranged in a spherical geometry to investigate and explain the effect of the dye-dye interactions [35] on the optical properties of a spherical shell of dye layer. It was later extended to Generalised CDM (GCDM), the case where those dye molecules are surrounding a nanoparticle (spherical core), to investigate the electromagnetic interactions between the adsorbed dye molecules and the metallic nano-sphere [37]. This GCDM can be used to study an array of inter-related effects: different molecular orientations, dye-dye interactions, self-reflected fields or image-dipole effect, and spatial distribution of adsorbates. However, the main shortcoming of the GCDM is the computing requirements. In a numerical simulation programme, MatLab, to solve for a 100 wavelength points spectrum may take from a few hours to a day on a high-end PC (for $10^{4}$ polarisable point dipoles on a sphere). This prevents systematic multi-parameter studies.

In contrast to the GCDM, Mie theory can be used to provide analytical solutions for a core-shell system (here both the core and shell media are linear, homogeneous, and isotropic). Mie theory can avoid the problem of high computational cost and complexity, which happens in the GCDM case, and it is much faster than GCDM. However, the presented Mie theory in this chapter is only for isotropic media, which cannot be used to study the effect of different molecular orientations of the dye molecules. To account for the effect of different molecular orientations of the dye molecules, we will need to treat the medium of the dye molecules' layer as an anisotropic medium (with an appropriate dielectric tensor to characterise the properties of the medium). Hence, the extension of Mie theory for anisotropic media is needed. 


\section{Mie Theory for Anisotropic Shell}

As we mentioned in the previous chapter, GCDM is more reliable than the isotropic Mie theory for solving light scattering problem by dye-coated nanoparticles, as we can make use of the GCDM to account for orientation effects of the dye-molecules. The GCDM is very time-consuming, especially for the cases involving large numbers of polarisable dipoles. Although it is more preferable than isotropic Mie theory, it leads to problems of the high computational cost and complexity which does not happen for Mie theory. The solution of Mie theory is commonly applied to isotropic media, where the dielectric function of the media are direction-independent. The isotropic assumptions are sometimes inadequate. Hence, the extension to anisotropic media has been developed, i.e. in the context of reflection/refraction at the interfaces [39]. The extension of Mie theory to radially anisotropic media is therefore needed, and this was developed almost 50 years ago by Roth and Dignam [40], where the dielectric functions describing the anisotropic media are in a tensor form. We can then use this extension of Mie theory (anisotropic Mie) to account for the molecular orientation effects.

This chapter is written based on the results of our paper Ref. [48]. We will here revisit the solutions of Mie theory for the light scattering problem by isotropic core/anisotropic shell (as done by Roth and Dignam [40]) with modern notations. The solutions to this problem involve the calculation of Bessel functions of complex orders, which are not built-in in many standard computing software (such as MatLab, for example). However, by assuming thin anisotropic shell approximation to the first order, the new solutions do not involve the Bessel functions of complex orders. The validity of this thin anisotropic shell approximation is assessed by comparing to the full solution of Anisotropic Mie theory (by Roth and Dignam [40]) and shown to agree extremely well for the shell's thickness of the order of $1 \mathrm{~nm}$ or less (typically, of a molecular monolayer). Overall, we have accounted for the anisotropy of the dye response in the Mie theory, which strong orientation and anisotropic effect are evidenced. The predictions of those are then compared with the GCDM (in which 
an individual dye is considered as a polarisable dipole). This work highlights the importance of the extension of the current isotropic model to anisotropic Mie theory, which can predict the orientation effects in plasmon-dye interactions.

\subsection{Electric and Magnetic Fields for Anisotropic Media}

\subsubsection{Maxwell's Equations}

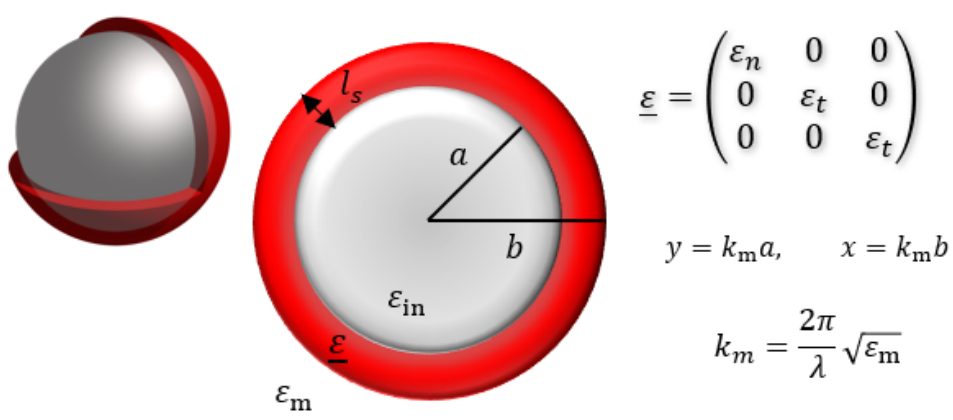

Figure 3.1: Schematic of the problem: a spherical core with a radius $a$ and dielectric function $\varepsilon_{\text {in }}$ is surrounded by an anisotropic shell with thickness $l_{s}=b-a$ described by dielectric tensor $\underline{\varepsilon}$. The system is embedded in a non-absorbing medium of dielectric constant $\varepsilon_{\mathrm{m}}$. The dielectric tensor $\underline{\varepsilon}$ is considered diagonal in spherical basis with its normal $\left(\varepsilon_{\mathrm{n}}\right)$ and tangential $\left(\varepsilon_{\mathrm{t}}\right)$ components.

For anisotropic media, Maxwell's equations take almost the same form as in the isotropic case. The difference between the two is that the dielectric function of the anisotropic media will be taken as a tensor and Eq. 2.1 will then be:

$$
\begin{array}{ll}
\nabla \cdot \mathbf{E}=0, & \nabla \cdot \mathbf{E}=0 \\
\nabla \times \mathbf{E}=i \omega \mu \mathbf{H}, & \nabla \times \mathbf{H}=-i \omega \underline{\underline{E}} .
\end{array}
$$

In this case, the Helmholtz equation cannot be satisfied by the fields, unlike in the isotropic case. For the anisotropic shell, we consider $\underline{\varepsilon}$ (diagonal in spherical basis and can be complex) to have its components $\varepsilon_{n}$ and $\varepsilon_{t}$ (as shown in Fig. 3.1), which correspond to the normal and tangential components of the $\mathbf{E}$-field, i.e.

$$
(\underline{\varepsilon} \mathbf{E})_{r}=\varepsilon_{\mathrm{n}} \mathrm{E}_{r}, \quad(\underline{\varepsilon} \mathbf{E})_{\theta}=\varepsilon_{\mathrm{t}} \mathrm{E}_{\theta}, \quad(\underline{\varepsilon} \mathbf{E})_{\phi}=\varepsilon_{\mathrm{t}} \mathrm{E}_{\phi}
$$

This will be useful later for describing the orientation effects for dye's molecules surrounding a nanoparticle. 


\subsubsection{Anisotropic VSHs}

The solutions to the anisotropic Maxwell equations can be represented as a superposition of the two linearly independent fields $\left(\mathbf{E}_{\mathrm{TM}}, \mathbf{H}_{\mathrm{TM}}\right)$ and $\left(\mathbf{E}_{\mathrm{TE}}, \mathbf{H}_{\mathrm{TE}}\right)$, which satisfy the conditions of the TM and TE waves. The next step to solving the anisotropic Maxwell equations is to construct vector functions from a scalar function $f$, similar to the isotropic case (section 2.1.2). We here construct the VSHs for anisotropic media as follows:

$$
\begin{array}{llll}
\mathbf{E}_{\mathrm{TE}} \propto \mathbf{M}=\nabla \times(\mathbf{r} f) & \& & \mathbf{H}_{\mathrm{TM}} \propto \tilde{\mathbf{M}}=\nabla \times(\mathbf{r} \tilde{f}) \\
\mathbf{H}_{\mathrm{TE}} \propto \mathbf{N}=\frac{\nabla \times \mathbf{M}}{k_{\mathrm{t}}} & \& & \underline{\varepsilon} \mathbf{E}_{\mathrm{TM}} \propto \tilde{\mathbf{N}}=\varepsilon_{\mathrm{t}} \frac{\nabla \times \tilde{\mathbf{M}}}{k_{\mathrm{t}}} .
\end{array}
$$

These constructions of $\mathbf{M} \& \mathbf{N}$, and $\tilde{\mathbf{M}} \& \tilde{\mathbf{N}}$ are to ensure that they satisfy the anisotropic Maxwell equations. By substituting Eqs. 3.2 and 3.3 into Eq. 3.1, we get:

$$
\begin{aligned}
& \frac{1}{r^{2}} \frac{\partial}{\partial r}\left(r^{2} \frac{\partial f}{\partial r}\right)+\frac{1}{r^{2} \sin \theta} \frac{\partial}{\partial \theta}\left(\sin \theta \frac{\partial f}{\partial \theta}\right)+\frac{1}{r^{2} \sin ^{2} \theta} \frac{\partial^{2} f}{\partial \phi^{2}}+k_{\mathrm{t}}^{2} f=0, \\
& \frac{\varepsilon_{\mathrm{n}}}{\varepsilon_{\mathrm{t}}} \frac{1}{r^{2}} \frac{\partial}{\partial r}\left(r^{2} \frac{\partial \tilde{f}}{\partial r}\right)+\frac{1}{r^{2} \sin \theta} \frac{\partial}{\partial \theta}\left(\sin \theta \frac{\partial \tilde{f}}{\partial \theta}\right)+\frac{1}{r^{2} \sin ^{2} \theta} \frac{\partial^{2} \tilde{f}}{\partial \phi^{2}}+k_{\mathrm{n}}^{2} \tilde{f}=0,
\end{aligned}
$$

where

$$
k_{u}^{2}=(\omega / c)^{2} \varepsilon_{u}, \quad(u=\mathrm{n}, \mathrm{t}) .
$$

The generating function $f$ for the TE wave is the same as in the isotropic case, with $\varepsilon$ being replaced by $\varepsilon_{\mathrm{t}}$. However, for the TM wave, the differential equation for its generating function $\tilde{f}$ is different from that of the TE wave by a factor $\varepsilon_{\mathrm{n}} / \varepsilon_{\mathrm{t}}$ in the first term. We are therefore required to solve Eq. 3.5 by making use of the separation of variable method again as we did for the isotropic case (Eqs. 2.8-2.15). We are seeking particular solutions of the form:

$$
\tilde{f}=\tilde{R}(r) \tilde{\Theta}(\theta) \tilde{\Phi}(\phi)
$$

By substituting this into Eq. 3.5, it yields:

$$
\begin{aligned}
\frac{d^{2} \tilde{\Phi}}{d \phi^{2}}+m^{2} \tilde{\Phi} & =0 \\
\frac{1}{\sin \theta} \frac{d}{d \theta}\left(\sin \theta \frac{d \tilde{\Theta}}{d \theta}\right)+\left(n(n+1)-\frac{m^{2}}{\sin ^{2} \theta}\right) \tilde{\Theta} & =0 \\
\frac{\varepsilon_{\mathrm{n}}}{\varepsilon_{\mathrm{t}}} \frac{d}{d r}\left(r^{2} \frac{d \tilde{R}}{d r}\right)+\left[k_{\mathrm{n}}^{2} r^{2}-n(n+1)\right] \tilde{R} & =0 .
\end{aligned}
$$


As we have seen in the isotropic case, the solutions to the first two equations (Eqs. 3.7 and 3.8) are the usual spherical harmonic functions, which are the sinusoids, $e^{i m \phi}$, the associated Legendre polynomials of $\cos \theta, P_{n}^{m}(\cos \theta)$, respectively. For the third equation, Eq. 3.9, with the substitution of $\rho=k_{t} r$, it then becomes:

$$
\frac{d}{d \rho}\left(\rho^{2} \frac{d \tilde{R}}{d \rho}\right)+\left[\rho^{2}-w(w+1)\right] \tilde{R}=0,
$$

where

$$
w(n)=\sqrt{\frac{1}{4}+\frac{\varepsilon_{\mathrm{t}}}{\varepsilon_{\mathrm{n}}}\left(n^{2}+n\right)}-\frac{1}{2} .
$$

Equation 3.10 is the same as Eq. 2.13 with $n$ replaced by $w(n)$, which can be complex and depends on the values of $\varepsilon_{\mathrm{n}}$ and $\varepsilon_{\mathrm{t}}$. Therefore, the solutions to Eq. 3.10 are the Bessel functions of complex order (their series expressions are presented in Appendix A). The VSHs for the anisotropic are therefore found as:

$$
\begin{aligned}
\tilde{\mathbf{M}}_{n m}^{(j)}(\rho, \theta, \phi) & =\mathcal{F}_{n m} \nabla \times\left(\mathbf{r} e^{i m \phi} P_{n}^{m}(\cos \theta) \tilde{z}_{n}^{(j)}(\rho)\right) \\
\tilde{\varepsilon}_{n m}^{(j)}(\rho, \theta, \phi) & =\varepsilon_{\mathrm{t}} \frac{\nabla \times \tilde{\mathbf{M}}_{n m}^{(j)}(\rho, \theta, \phi)}{k_{\mathrm{t}}}
\end{aligned}
$$

where $\mathcal{F}_{n m}$ is given in Eq. 2.15. [NB: we will use the notation $\tilde{z}_{n}^{(j)}(\rho)$ [instead of $\left.z_{w}^{(j)}(\rho)\right]$, i.e. $\tilde{z}_{n} \equiv z_{w}$, to represent the solutions, which are the Bessel functions of complex orders.] Therefore, the fields solutions inside an anisotropic medium can be expanded as an infinite series of a complete set of VSHs and hence can be written as:

$$
\begin{aligned}
& \mathbf{E}_{\text {Aniso }}=\frac{\underline{\varepsilon}}{\varepsilon_{\mathrm{t}}} E_{0} \sum_{n, m} \alpha_{n m} \mathbf{M}_{n m}^{(1)}\left(k_{\mathrm{t}} \mathbf{r}\right)+\beta_{n m} \tilde{\mathbf{N}}_{n m}^{(1)}\left(k_{\mathrm{t}} \mathbf{r}\right)+\gamma_{n m} \mathbf{M}_{n m}^{(2)}\left(k_{\mathrm{t}} \mathbf{r}\right)+\delta_{n m} \tilde{\mathbf{N}}_{n m}^{(2)}\left(k_{\mathrm{t}} \mathbf{r}\right), \\
& \mathbf{H}_{\text {Aniso }}=\frac{k_{\mathrm{t}}}{i \omega \mu} E_{0} \sum_{n, m} \alpha_{n m} \mathbf{N}_{n m}^{(1)}\left(k_{\mathrm{t}} \mathbf{r}\right)+\beta_{n m} \tilde{\mathbf{M}}_{n m}^{(1)}\left(k_{\mathrm{t}} \mathbf{r}\right)+\gamma_{n m} \mathbf{N}_{n m}^{(2)}\left(k_{\mathrm{t}} \mathbf{r}\right)+\delta_{n m} \tilde{\mathbf{M}}_{n m}^{(2)}\left(k_{\mathrm{t}} \mathbf{r}\right) .
\end{aligned}
$$

\subsection{Scattering by Anisotropic Shell/Isotropic Core}

In this section, we consider a dielectric sphere with a radius $a$ and a dielectric function $\varepsilon_{\text {in }}$, which is located at the origin $O$ and surrounded by an anisotropic shell with thickness $b-a$ and dielectric tensor $\underline{\varepsilon}_{\mathrm{s}}$. The system is considered to be embedded in a medium with dielectric function $\varepsilon_{\mathrm{m}}$. The corresponding wave vectors 
in all the media are given as:

$$
k_{\text {in }}=\sqrt{\varepsilon_{\text {in }}} \frac{\omega}{c}, \quad k_{\mathrm{m}}=\sqrt{\varepsilon_{\mathrm{m}}} \frac{\omega}{c}, \quad k_{\mathrm{t}}=\sqrt{\varepsilon_{\mathrm{t}}} \frac{\omega}{c},
$$

where $\varepsilon_{\mathrm{t}}$ is the tangential component of $\underline{\varepsilon}_{\mathrm{s}}$. The incident, scattered, and internal fields can be defined in the previous chapter (Eqs. 2.24-2.23). The fields inside the anisotropic shell can be expanded in terms of VSHs as in Eqs. 3.14 and 3.15.

\subsubsection{Boundary Conditions and The Susceptibilities}

Since our scattering problem is by a core-shell system, there will be boundary conditions to be applied at $r=a$ and $r=b$.

At $r=a$, the boundary conditions are:

$$
\begin{aligned}
& \mathbf{E}_{\text {Aniso }} \cdot \hat{\theta}=\mathbf{E}_{\text {in }} \cdot \hat{\theta} \\
& \mathbf{E}_{\text {Aniso }} \cdot \hat{\phi}=\mathbf{E}_{\text {in }} \cdot \hat{\phi} \\
& \mathbf{H}_{\text {Aniso }} \cdot \hat{\theta}=\mathbf{H}_{\text {in }} \cdot \hat{\theta} \\
& \mathbf{H}_{\text {Aniso }} \cdot \hat{\phi}=\mathbf{H}_{\text {in }} \cdot \hat{\phi}
\end{aligned}
$$

At $r=b$, the boundary conditions are:

$$
\begin{array}{r}
\left(\mathbf{E}_{\text {Inc }}+\mathbf{E}_{\text {Sca }}\right) \cdot \hat{\theta}=\mathbf{E}_{\text {Aniso }} \cdot \hat{\theta} \\
\left(\mathbf{E}_{\text {Inc }}+\mathbf{E}_{\text {Sca }}\right) \cdot \hat{\phi}=\mathbf{E}_{\text {Aniso }} \cdot \hat{\phi} \\
\left(\mathbf{H}_{\text {Inc }}+\mathbf{H}_{\text {Sca }}\right) \cdot \hat{\theta}=\mathbf{H}_{\text {Aniso }} \cdot \hat{\theta} \\
\left(\mathbf{H}_{\text {Inc }}+\mathbf{H}_{\text {Sca }}\right) \cdot \hat{\phi}=\mathbf{H}_{\text {Aniso }} \cdot \hat{\phi} .
\end{array}
$$

By solving these boundary conditions, it will lead to the same expressions in Eqs. 2.29 and 2.30. The scattered susceptibilities $\Gamma_{n}$ and $\Delta_{n}$ can be found by applying the above boundary conditions. We shall now apply the boundary conditions at $r=a$, and get:

$$
\begin{aligned}
{\left[\alpha_{n m} \psi_{n}\left(s_{\mathrm{t}} y\right)+\gamma_{n m} \chi_{n}\left(s_{\mathrm{t}} y\right)\right] / s_{\mathrm{t}} } & =c_{n m} \psi_{n}\left(s_{\mathrm{in}} y\right) / s_{\mathrm{in}} \\
{\left[\beta_{n m} \tilde{\psi}_{n}^{\prime}\left(s_{\mathrm{t}} y\right)+\delta_{n m} \tilde{\chi}_{n}^{\prime}\left(s_{\mathrm{t}} y\right)\right] / s_{\mathrm{t}} } & =d_{n m} \psi_{n}^{\prime}\left(s_{\text {in }} y\right) / s_{\text {in }} \\
\alpha_{n m} \psi_{n}^{\prime}\left(s_{\mathrm{t}} y\right)+\gamma_{n m} \chi_{n}^{\prime}\left(s_{\mathrm{t}} y\right) & =c_{n m} \psi_{n}^{\prime}\left(s_{\mathrm{in}} y\right) \\
\beta_{n m} \tilde{\psi}_{n}\left(s_{\mathrm{t}} y\right)+\delta_{n m} \tilde{\chi}_{n}\left(s_{\mathrm{t}} y\right) & =d_{n m} \psi_{n}\left(s_{\text {in }} y\right),
\end{aligned}
$$

where $s_{\mathrm{t}}=\sqrt{\varepsilon_{\mathrm{t}}} / \sqrt{\varepsilon_{\mathrm{m}}}, s_{\text {in }}=\sqrt{\varepsilon_{\mathrm{in}}} / \sqrt{\varepsilon_{\mathrm{m}}}, y=k_{\mathrm{m}} a$, and $\chi_{n}(x)=x y_{n}(x)$. These 
equations will then lead to:

$$
\begin{aligned}
& \frac{\gamma_{n m}}{\alpha_{n m}}=-\frac{s_{\mathrm{t}} \psi_{n}^{\prime}\left(s_{\mathrm{t}} y\right) \psi_{n}\left(s_{\text {in }} y\right)-s_{\text {in }} \psi_{n}\left(s_{\mathrm{t}} y\right) \psi_{n}^{\prime}\left(s_{\text {in }} y\right)}{s_{\mathrm{t}} \chi_{n}^{\prime}\left(s_{\mathrm{t}} y\right) \psi_{n}\left(s_{\mathrm{in}} y\right)-s_{\text {in }} \chi_{n}\left(s_{\mathrm{t}} y\right) \psi_{n}^{\prime}\left(s_{\mathrm{in}} y\right)} \equiv \Gamma_{n}^{(1)} \\
& \frac{\delta_{n m}}{\beta_{n m}}=-\frac{s_{\mathrm{t}} \tilde{\psi}_{n}\left(s_{\mathrm{t}} y\right) \psi_{n}^{\prime}\left(s_{\text {in }} y\right)-s_{\text {in }} \tilde{\psi}_{n}^{\prime}\left(s_{\mathrm{t}} y\right) \psi_{n}\left(s_{\text {in }} y\right)}{s_{\mathrm{t}} \tilde{\chi}_{n}\left(s_{\mathrm{t}} y\right) \psi_{n}^{\prime}\left(s_{\text {in }} y\right)-s_{\text {in }} \tilde{\chi}_{n}^{\prime}\left(s_{\mathrm{t}} y\right) \psi_{n}\left(s_{\text {in }} y\right)} \equiv \Delta_{n}^{(1)} .
\end{aligned}
$$

Boundary conditions at $r=b$ gives:

$$
\begin{aligned}
a_{n m} \psi_{n}(x)+p_{n m} \xi_{n}(x) & =\left[\alpha_{n m} \psi_{n}\left(s_{\mathrm{t}} x\right)+\gamma_{n m} \chi_{n}\left(s_{\mathrm{t}} x\right)\right] / s_{\mathrm{t}} \\
b_{n m} \psi_{n}^{\prime}(x)+q_{n m} \xi_{n}^{\prime}(x) & =\left[\beta_{n m} \tilde{\psi}_{n}^{\prime}\left(s_{\mathrm{t}} x\right)+\delta_{n m} \tilde{\chi}_{n}^{\prime}\left(s_{\mathrm{t}} x\right)\right] / s_{\mathrm{t}} \\
a_{n m} \psi_{n}^{\prime}(x)+p_{n m} \xi_{n}^{\prime}(x) & =\alpha_{n m} \psi_{n}^{\prime}\left(s_{\mathrm{t}} x\right)+\gamma_{n m} \chi_{n}^{\prime}\left(s_{\mathrm{t}} x\right) \\
b_{n m} \psi_{n}(x)+q_{n m} \xi_{n}(x) & =\beta_{n m} \tilde{\psi}_{n}\left(s_{\mathrm{t}} x\right)+\delta_{n m} \tilde{\chi}_{n}\left(s_{\mathrm{t}} x\right),
\end{aligned}
$$

where $x=k_{\mathrm{m}} b$. These equations together with Eqs. 3.25 and 3.26, it yields:

$$
\begin{aligned}
\Gamma_{n} & =-\frac{[\psi \psi]_{n, x}^{m, s}[\chi \psi]_{n, y}^{m, c}-[\chi \psi]_{n, x}^{m, s}[\psi \psi]_{n, y}^{m, c}}{[\psi \xi]_{n, x}^{m, s}[\chi \psi]_{n, y}^{m, c}-[\chi \xi]_{n, x}^{m, s}[\psi \psi]_{n, c}^{m, c}} \\
\Delta_{n} & =-\frac{[\psi \psi]_{n, x}^{e, s}[\chi \psi]_{n, y}^{e, c}-[\chi \psi]_{n, x}^{e, s}[\psi \psi]_{n, c}^{e, c}}{[\psi \xi]_{n, x}^{e, s}[\chi \psi]_{n, y}^{e, c}-[\chi \xi]_{n, x}^{e, s}[\psi \psi]_{n, y}^{e, c}}
\end{aligned}
$$

where

$$
\begin{aligned}
& {[\Lambda \psi]_{n, y}^{m, c}=s_{\mathrm{in}} \Lambda_{n}\left(s_{\mathrm{t}} y\right) \psi_{n}^{\prime}\left(s_{\mathrm{in}} y\right)-s_{\mathrm{t}} \Lambda_{n}^{\prime}\left(s_{\mathrm{t}} y\right) \psi_{n}\left(s_{\mathrm{in}} y\right),} \\
& {[\Lambda \psi]_{n, y}^{e, c}=s_{\mathrm{in}} \tilde{\Lambda}_{n}^{\prime}\left(s_{\mathrm{t}} y\right) \psi_{n}\left(s_{\mathrm{in}} y\right)-s_{\mathrm{t}} \tilde{\Lambda}_{n}\left(s_{\mathrm{t}} y\right) \psi_{n}^{\prime}\left(s_{\mathrm{in}} y\right),} \\
& {[\Lambda \Omega]_{n, x}^{m, s}=s_{\mathrm{t}} \Lambda_{n}^{\prime}\left(s_{\mathrm{t}} x\right) \Omega_{n}(x)-\Lambda_{n}\left(s_{\mathrm{t}} x\right) \Omega_{n}^{\prime}(x),} \\
& {[\Lambda \Omega]_{n, x}^{e, s}=s_{\mathrm{t}} \tilde{\Lambda}_{n}\left(s_{\mathrm{t}} x\right) \Omega_{n}^{\prime}(x)-\tilde{\Lambda}_{n}^{\prime}\left(s_{\mathrm{t}} x\right) \Omega_{n}(x),} \\
& \text { with, } \Lambda=\psi \text { or } \chi, \text { and } \Omega=\psi \text { or } \xi .
\end{aligned}
$$

These solutions to the problem were fully derived in Ref. [40], and the summary of that is shown above. The extinction, scattering, and absorption cross-sections, in particular, will take the same form as in isotropic Mie theory Eqs. 2.41-2.43.

\subsubsection{Thin Anisotropic Shell Approximations}

Since the solutions of the problem involve the calculations of Bessel functions of complex orders, previous studies by Lange and Aragón [57] have simplified the solution by making assumptions of thin anisotropic shell approximation in the special case of bubbles and vesicles (where $\varepsilon_{\mathrm{in}}=\varepsilon_{\mathrm{m}}$ ), where the complex-ordered Bessel functions are no longer required. That work was later extended to a thicker shell by including the next order corrections [58]. In the following, we generalise the results of the thin anisotropic shell to the case where the core is different from 
the outside medium, $\varepsilon_{\text {in }} \neq \varepsilon_{\mathrm{m}}$. We will go through step by step of how this thin anisotropic shell approximation is derived and compare the results to those from the full anisotropic Mie scattering (whose susceptibilities defined in Eqs. 3.27 and 3.28).

For this thin anisotropic shell approximation (TASA), we will use Taylor's expansion to the first order in $\delta=x-y=k_{\mathrm{m}}(b-a)=k_{\mathrm{m}} l_{\mathrm{s}}$, in the limit $\delta \ll x, y$. Therefore, we can view the Mie coefficients as functions of $x$ and $y$, or equivalently $y$ and $\delta$. Using Taylor's expansion to the first order, $\Gamma_{n}$ and $\Delta_{n}$ can be written as:

$$
\begin{aligned}
& \Gamma_{n}(x, y) \approx \Gamma_{n}(x=y, y)+\left.\delta \frac{\partial \Gamma_{n}}{\partial x}\right|_{x=y}, \\
& \Delta_{n}(x, y) \approx \Delta_{n}(x=y, y)+\left.\delta \frac{\partial \Delta_{n}}{\partial x}\right|_{x=y}
\end{aligned}
$$

We shall first rewrite $\Gamma_{n}$ and $\Delta_{n}$ into their numerators and denominators as:

$$
\Gamma_{n}=-\frac{N_{1 n}}{D_{1 n}}, \quad \Delta_{n}=-\frac{N_{2 n}}{D_{2 n}}
$$

Then, the Taylor expansions in Eqs 3.30 and 3.31 become:

$$
\begin{aligned}
& \Gamma_{n}(\delta)=-\left.\frac{N_{1 n}}{D_{1 n}}\right|_{x=y}-\left.\delta\left(\frac{N_{1 n}^{\prime}}{D_{1 n}}-\frac{N_{1 n} D_{1 n}^{\prime}}{D_{1 n}^{2}}\right)\right|_{x=y}, \\
& \Delta_{n}(\delta)=-\left.\frac{N_{2 n}}{D_{2 n}}\right|_{x=y}-\left.\delta\left(\frac{N_{2 n}^{\prime}}{D_{2 n}}-\frac{N_{2 n} D_{2 n}^{\prime}}{D_{2 n}^{2}}\right)\right|_{x=y} .
\end{aligned}
$$

The numerators, $N_{1 n}, N_{2 n}$, the denominators, $D_{1 n}, D_{2 n}$ and their corresponding first derivatives (denoted with ') with respect to $x$ are therefore needed. To do so, we will need to make use of some useful identities below. The first useful identity is the relationship (differential equations) between the generalised Riccati-Bessel functions and their second derivatives, which are derived from Eqs 2.12 and 3.9. These were also obtained by Lange and Aragón (Eqs. A9 and A10 in Ref. [57]):

$$
\begin{aligned}
& \Xi_{n}^{\prime \prime}+\left[1-\frac{n(n+1)}{x^{2}}\right] \Xi_{n}=0, \\
& \tilde{\Xi}_{n}^{\prime \prime}+\left[1-\frac{\varepsilon_{\mathrm{t}}}{\varepsilon_{\mathrm{n}}} \frac{n(n+1)}{x^{2}}\right] \tilde{\Xi}_{n}=0,
\end{aligned}
$$

where $\Xi$ represents all types of the Riccati-Bessel functions $\psi, \chi$, and $\xi$. Two useful identities can be found in Ref. [57] (Eqs. C1 and C2), which are written below together with other identities of similar form that will be needed for our derivations 
of TASA.

$$
\begin{aligned}
& {[\psi \Omega]_{n, y}^{e, s} \tilde{\chi}_{n}\left(s_{\mathrm{t}} y\right)-[\chi \Omega]_{n, y}^{e, s} \tilde{\psi}_{n}\left(s_{\mathrm{t}} y\right)=\Omega_{n}(y),} \\
& {[\psi \Omega]_{n, y}^{e, s} \tilde{\chi}_{n}^{\prime}\left(s_{\mathrm{t}} y\right)-[\chi \Omega]_{n, y}^{e, s} \tilde{\psi}_{n}^{\prime}\left(s_{\mathrm{t}} y\right)=s_{\mathrm{t}} \Omega_{n}^{\prime}(y),} \\
& {[\psi \Omega]_{n, y}^{m, s} \chi_{n}\left(s_{\mathrm{t}} y\right)-[\chi \Omega]_{n, y}^{m, s} \psi_{n}\left(s_{\mathrm{t}} y\right)=-s_{\mathrm{t}} \Omega_{n}(y),} \\
& {[\psi \Omega]_{n, y}^{m, s} \chi_{n}^{\prime}\left(s_{\mathrm{t}} y\right)-[\chi \Omega]_{n, y}^{m, s} \psi_{n}^{\prime}\left(s_{\mathrm{t}} y\right)=-\Omega_{n}^{\prime}(y),} \\
& {[\psi \Omega]_{n, y}^{e, c} \tilde{\chi}_{n}\left(s_{\mathrm{t}} y\right)-[\chi \Omega]_{n, y}^{e, c} \tilde{\psi}_{n}\left(s_{\mathrm{t}} y\right)=-s_{\mathrm{in}} \Omega_{n}\left(s_{\mathrm{in}} y\right),} \\
& {[\psi \Omega]_{n, y}^{e, c} \tilde{\chi}_{n}^{\prime}\left(s_{\mathrm{t}} y\right)-[\chi \Omega]_{n, y}^{e, c} \tilde{\psi}_{n}^{\prime}\left(s_{\mathrm{t}} y\right)=-s_{\mathrm{t}} \Omega_{n}^{\prime}\left(s_{\mathrm{in}} y\right),} \\
& {[\psi \Omega]_{n, y}^{m, c} \chi_{n}\left(s_{\mathrm{t}} y\right)-[\chi \Omega]_{n, y}^{m, c} \psi_{n}\left(s_{\mathrm{t}} y\right)=s_{\mathrm{t}} \Omega_{n}\left(s_{\mathrm{in}} y\right),} \\
& {[\psi \Omega]_{n, y}^{m, c} \chi_{n}^{\prime}\left(s_{\mathrm{t}} y\right)-[\chi \Omega]_{n, y}^{m, c} \psi_{n}^{\prime}\left(s_{\mathrm{t}} y\right)=s_{\mathrm{in}} \Omega_{n}^{\prime}\left(s_{\mathrm{in}} y\right),}
\end{aligned}
$$

where $\Omega$ denotes $\psi$ or $\xi$ and the brackets [.] are defined in Eqs. 3.29. The first expression Eq. 3.37 can be easily proven as follows:

$$
\begin{aligned}
{[\psi \Omega \Omega]_{n, y}^{e, s} } & \tilde{\chi}_{n}\left(s_{\mathrm{t}} y\right)-[\chi \Omega]_{n, y}^{e, s} \tilde{\psi}_{n}\left(s_{\mathrm{t}} y\right) \\
& =\left\{s_{\mathrm{t}} \tilde{\psi}_{n}\left(s_{\mathrm{t}} y\right) \Omega_{n}^{\prime}(y)-\tilde{\psi}_{n}^{\prime}\left(s_{\mathrm{t}} y\right) \Omega_{n}(y)\right\} \tilde{\chi}_{n}\left(s_{\mathrm{t}} y\right)-\left\{s_{\mathrm{t}} \tilde{\chi}_{n}\left(s_{\mathrm{t}} y\right) \Omega_{n}^{\prime}(y)-\tilde{\chi}_{n}^{\prime}\left(s_{\mathrm{t}} y\right) \Omega_{n}(y)\right\} \tilde{\psi}_{n}\left(s_{\mathrm{t}} y\right) \\
& =s_{\mathrm{t}} \Omega_{n}^{\prime}(y)\left\{\tilde{\psi}_{n}\left(s_{\mathrm{t}} y\right) \tilde{\chi}_{n}\left(s_{\mathrm{t}} y\right)-\tilde{\chi}_{n}\left(s_{\mathrm{t}} y\right) \tilde{\psi}_{n}\left(s_{\mathrm{t}} y\right)\right\}-\Omega_{n}(y)\left\{\tilde{\psi}_{n}^{\prime}\left(s_{\mathrm{t}} y\right) \tilde{\chi}_{n}\left(s_{\mathrm{t}} y\right)-\tilde{\chi}_{n}^{\prime}\left(s_{\mathrm{t}} y\right) \tilde{\psi}_{n}\left(s_{\mathrm{t}} y\right)\right\} \\
& =\Omega_{n}(y),
\end{aligned}
$$

where for the last equality, we apply the Wronskian identity of the Riccati-Bessel function of the complex order [52], $\tilde{\psi}_{n} \tilde{\chi}_{n}^{\prime}-\tilde{\psi}_{n}^{\prime} \tilde{\chi}_{n}=1$. Then the other expressions Eqs. 3.38 - 3.44 can be easily proven in a similar fashion.

We now shall have a look at the first-order approximation of $\Delta_{n}(\delta)$ in Eq. 3.31. We will try to simplify each term to their simplest expressions (by applying the useful identities provided above) as follows:

$$
\begin{aligned}
\left.N_{2 n}\right|_{x=y}= & {[\psi \psi]_{n, y}^{e, s}[\chi \psi]_{n, y}^{e, c}-[\chi \psi]_{n, y}^{e, s}[\psi \psi]_{n, y}^{e, c} } \\
= & {[\psi \psi]_{n, y}^{e, s}\left\{s_{\text {in }} \tilde{\chi}_{n}^{\prime}\left(s_{\mathrm{t}} y\right) \psi_{n}\left(s_{\mathrm{in}} y\right)-s_{\mathrm{t}} \tilde{\chi}_{n}\left(s_{\mathrm{t}} y\right) \psi_{n}^{\prime}\left(s_{\mathrm{c}} y\right)\right\} } \\
& -[\chi \psi]_{n, y}^{e, s}\left\{s_{\mathrm{in}} \tilde{\psi}_{n}^{\prime}\left(s_{\mathrm{t}} y\right) \psi_{n}\left(s_{\mathrm{in}} y\right)-s_{\mathrm{t}} \tilde{\psi}_{n}\left(s_{\mathrm{t}} y\right) \psi_{n}^{\prime}\left(s_{\mathrm{in}} y\right)\right\} \\
= & s_{\mathrm{in}} \psi_{n}\left(s_{\mathrm{in}} y\right)\left\{[\psi \psi]_{n, y}^{e, s} \tilde{\chi}_{n}^{\prime}\left(s_{\mathrm{t}} y\right)-[\chi \psi]_{n, y}^{e, s} \tilde{\psi}_{n}^{\prime}\left(s_{\mathrm{t}} y\right)\right\} \\
& -s_{\mathrm{t}} \psi_{n}^{\prime}\left(s_{\mathrm{in}} y\right)\left\{[\psi \psi]_{n, y}^{e, s} \tilde{\chi}_{n}\left(s_{\mathrm{t}} y\right)-[\chi \psi]_{n, y}^{e, s} \tilde{\psi}_{n}\left(s_{\mathrm{t}} y\right)\right\} \\
= & s_{\mathrm{t}}\left\{s_{\text {in }} \psi_{n}\left(s_{\mathrm{in}} y\right) \psi_{n}^{\prime}(y)-\psi_{n}^{\prime}\left(s_{\text {in }} y\right) \psi_{n}(y)\right\} .
\end{aligned}
$$

For the last equality, we use the Eqs. 3.38 and 3.39. Similarly, the denominator $\left.D_{2 n}\right|_{x=y}$ can be found to be:

$$
\begin{aligned}
\left.D_{2 n}\right|_{x=y} & =[\psi \xi]_{n, y}^{e, s}[\chi \psi]_{n, y}^{e, c}-[\chi \xi]_{n, y}^{e, s}[\psi \psi]_{n, y}^{e, c} \\
& =s_{\mathrm{t}}\left\{s_{\mathrm{in}} \psi_{n}\left(s_{\mathrm{in}} y\right) \xi_{n}^{\prime}(y)-\psi_{n}^{\prime}\left(s_{\mathrm{in}} y\right) \xi_{n}(y)\right\} .
\end{aligned}
$$


We now still have to look at the first derivatives with respect to $x$ of $N_{2 n}(y, x)$ and $D_{2 n}(y, x)$.

$$
\begin{aligned}
& N_{2 n}^{\prime}(y, x)=\frac{d}{d x}\left([\psi \psi]_{n, x}^{e, s}\right)[\chi \psi]_{n, y}^{e, c}-\frac{d}{d x}\left([\chi \psi]_{n, x}^{e, s}\right)[\psi \psi]_{n, y}^{e, c} \\
& D_{2 n}^{\prime}(y, x)=\frac{d}{d x}\left([\psi \xi]_{n, x}^{e, s}\right)[\chi \psi]_{n, y}^{e, c}-\frac{d}{d x}\left([\chi \xi]_{n, x}^{e, s}\right)[\psi \psi]_{n, y}^{e, c},
\end{aligned}
$$

with

$$
\frac{d}{d x}\left([\Lambda \Omega]_{n, x}^{e, s}\right)=s_{\mathrm{t}}^{2} \tilde{\Lambda}_{n}^{\prime}\left(s_{\mathrm{t}} x\right) \Omega_{n}^{\prime}(x)+s_{\mathrm{t}} \tilde{\Lambda}_{n}\left(s_{\mathrm{t}} x\right) \Omega_{n}^{\prime \prime}(x)-s_{\mathrm{t}} \tilde{\Lambda}_{n}^{\prime \prime}\left(s_{\mathrm{t}} x\right) \Omega_{n}(x)-\tilde{\Lambda}_{n}^{\prime}\left(s_{\mathrm{t}} x\right) \Omega_{n}^{\prime}(x) .
$$

Then, we use Eq. 3.36 to replace the second derivatives, which leads to:

$$
\left.\frac{d}{d x}\left([\Lambda \Omega]_{n, x}^{e, s}\right)\right|_{x=y}=\frac{s_{\mathrm{t}} n(n+1)}{y^{2}}\left(1-\frac{\varepsilon_{\mathrm{t}}}{\varepsilon_{\mathrm{n}} s_{\mathrm{t}}^{2}}\right) \tilde{\Lambda}_{n}\left(s_{\mathrm{t}} y\right) \Omega_{n}(y)+\left(s_{\mathrm{t}}^{2}-1\right) \tilde{\Lambda}_{n}^{\prime}\left(s_{\mathrm{t}} y\right) \Omega_{n}^{\prime}(y) .
$$

We now can make use of Eqs. 3.42 and 3.43 to substitute into Eq. 3.50 to get:

$$
\left.N_{2 n}^{\prime}\right|_{x=y}=\frac{s_{\mathrm{t}} s_{\mathrm{in}} n(n+1)}{y^{2}}\left(1-\frac{\varepsilon_{\mathrm{t}}}{\varepsilon_{\mathrm{n}} s_{\mathrm{t}}^{2}}\right) \psi_{n}(y) \psi_{n}\left(s_{\mathrm{in}} y\right)+s_{\mathrm{t}}\left(s_{\mathrm{t}}^{2}-1\right) \psi_{n}^{\prime}(y) \psi_{n}^{\prime}\left(s_{\text {in }} y\right) .
$$

By applying similar derivations, we can also obtain:

$$
\left.D_{2 n}^{\prime}\right|_{x=y}=\frac{s_{\mathrm{t}} s_{\mathrm{in}} n(n+1)}{y^{2}}\left(1-\frac{\varepsilon_{\mathrm{t}}}{\varepsilon_{\mathrm{n}} s_{\mathrm{t}}^{2}}\right) \xi_{n}(y) \psi_{n}\left(s_{\mathrm{in}} y\right)+s_{\mathrm{t}}\left(s_{\mathrm{t}}^{2}-1\right) \xi_{n}^{\prime}(y) \psi_{n}^{\prime}\left(s_{\mathrm{in}} y\right) .
$$

For the derivation of $\Gamma_{n}$, we can apply similar procedures using Eqs. 3.39, 3.40, 3.43, and 3.44, which yields:

$$
\begin{aligned}
& \left.N_{1 n}\right|_{x=y}=s_{\mathrm{t}}\left\{s_{\text {in }} \psi_{n}(y) \psi_{n}^{\prime}\left(s_{\text {in }} y\right)-\psi_{n}^{\prime}(y) \psi_{n}\left(s_{\text {in }} y\right)\right\} \\
& \left.D_{1 n}\right|_{x=y}=s_{\mathrm{t}}\left\{s_{\text {in }} \xi_{n}(y) \psi_{n}^{\prime}\left(s_{\text {in }} y\right)-\xi_{n}^{\prime}(y) \psi_{n}\left(s_{\text {in }} y\right)\right\} \\
& \left.N_{1 n}^{\prime}\right|_{x=y}=s_{\mathrm{t}}\left(1-s_{\mathrm{t}}^{2}\right) \psi_{n}(y) \psi_{n}\left(s_{\text {in }} y\right) \\
& \left.D_{1 n}^{\prime}\right|_{x=y}=s_{\mathrm{t}}\left(1-s_{\mathrm{t}}^{2}\right) \xi_{n}(y) \psi_{n}\left(s_{\text {in }} y\right) .
\end{aligned}
$$

Note that if the thickness of the shell $\delta=0$, the expressions of $\Gamma_{n}$ and $\Delta_{n}$ will reduce to the solutions of the standard Mie theory for a single sphere, as one would expect. The most important outcome of this TASA (first-order Taylor expansion) is that the derived expressions for both $\Gamma_{n}$ and $\Delta_{n}$ now no longer involve spherical Bessel functions of complex order. They only require the usual spherical Bessel functions of order $n$, as in standard Mie theory. Our TASA is a more generalised 
result than the thin shell approximation for bubbles in Ref. [57]. In fact, if we set the inside medium $\varepsilon_{\text {in }}=\varepsilon_{\mathrm{m}}$ (i.e. $s_{\text {in }}=1$ ), then our expressions for $\Gamma_{n}$ and $\Delta_{n}$ will further reduce to:

$$
\begin{aligned}
& \Gamma_{n}(\delta)=i \delta\left(1-s_{\mathrm{t}}^{2}\right)\left\{\psi_{n}(y)\right\}^{2} \\
& \Delta_{n}(\delta)=i \delta\left[\frac{n(n+1)}{y^{2}}\left(\frac{\varepsilon_{\mathrm{t}}}{\varepsilon_{\mathrm{n}} s_{\mathrm{t}}^{2}}-1\right)\left\{\psi_{n}(y)\right\}^{2}+\left(1-s_{\mathrm{t}}^{2}\right)\left\{\psi_{n}^{\prime}(y)\right\}^{2}\right],
\end{aligned}
$$

which are exactly the same expressions as (Eqs. 3.16 and 3.17) in Ref. [57], as expected.

\subsection{Validation of TASA Model and Application}

The advantage of the TASA model is that the Bessel functions of complex orders dropped out and only the regular Bessel functions remain in the solutions of the scattering coefficients. In this section, we would like to check the validity of the TASA model, which requires a few quantities (to be defined, e.g. dielectric functions for the anisotropic shell) for our MatLab simulation and to compare the results with those of the full anisotropic Mie model. To derive the dielectric functions for the anisotropic shell, we will use microscopic and effective medium models as a guide to account for different orientations of the dye molecules, in the dilute regime.

In order to demonstrate the range of validity and usefulness of this TASA, we will focus on a specific system: a dye-coated metallic nanosphere. The study on such structures have been investigated to measure the extinction and absorption properties [34], and they are relevant to many experimental techniques, such as SERS or fluorescence [27], and to the studies of molecular/plasmon resonance interactions. Also, the results in the surface selection rules in SERS [27, 59, 60, 61] have shown that the adsorption orientation of the dye molecules on a metallic nanoparticle can have significant effects on the optical properties of the whole system. 


\subsubsection{Microscopic Model for an Anisotropic Layer of Molecules Ad- sorbed on a Metal Sphere}
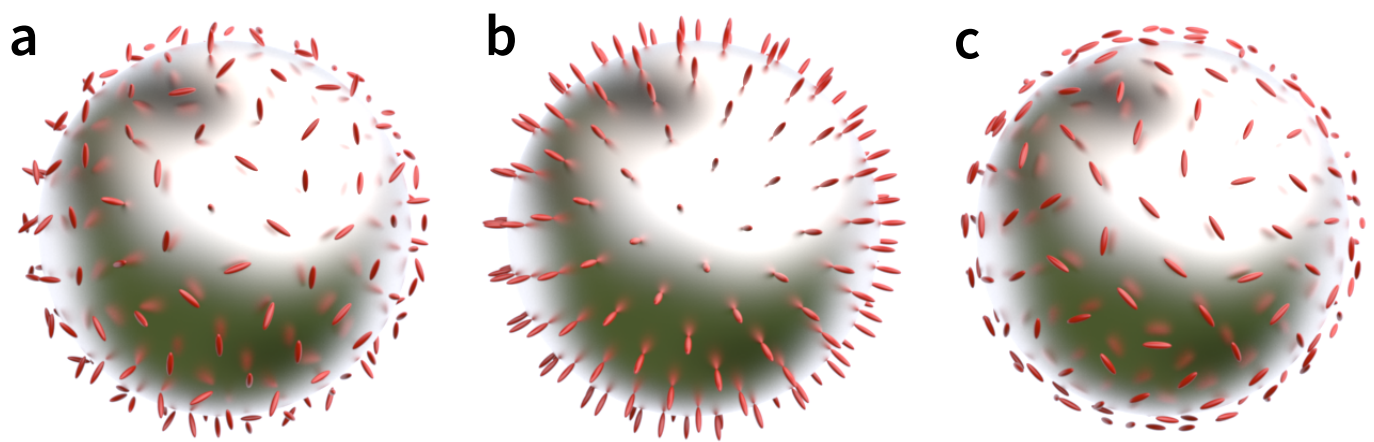

Figure 3.2: We are interested in three possible adsorptions of uniaxial dyes relevant to experiments: (a) fully random orientation denoted $*$, (b) perpendicular or radial denoted $\perp$, and (c) randomly oriented in-plane or tangential to the surface denoted $\|$.

We will first look at an isolated dye molecule on a metallic nanosphere, then use that result to extend to $N$ dye molecules. By using a microscopic description of a single dye adsorbed onto a metallic nanosphere and Mie theory for the nanosphere, its optical properties can then be understood. For a given incident electric field $\mathbf{E}_{0}$, the local electric field at the dye position $\mathbf{E}_{\text {loc }}$ can be easily computed from Mie theory and can be split into a sum of its normal and tangential components (w.r.t the sphere surface), $\mathbf{E}_{\text {loc }}^{\perp}$ and $\mathbf{E}_{\text {loc }}^{\|}$, respectively. For a dye molecule with polarisability tensor $\underline{\alpha}$, the local field then induces an oscillating dipole:

$$
\mathbf{p}=\underline{\alpha} \mathbf{E}_{\text {loc }}
$$

The dye polarisability tensor is commonly considered to be uniaxial along the main molecular axis (with unit vector $\hat{\mathbf{e}}_{\mathrm{d}}$ ), and it is characterised by the scalar polarisability along that axis, $\alpha_{z z}$. The induced dipole then becomes:

$$
\mathbf{p}=\alpha_{z z}\left(\hat{\mathbf{e}}_{\mathrm{d}} \cdot \mathbf{E}_{\mathrm{loc}}\right) \hat{\mathbf{e}}_{\mathrm{d}} .
$$

The absorption cross-section for this induced dipole moment can therefore be expressed as written in Eq. 2.78:

$$
\begin{aligned}
\sigma_{\mathrm{Abs}} & =\frac{2 \pi}{\lambda \varepsilon_{0} \sqrt{\varepsilon_{\mathrm{m}}}} L_{\mathrm{m}}^{2} \frac{\Im\left[\mathbf{p} \cdot \mathbf{E}_{\mathrm{loc}}^{*}\right]}{\left|\mathbf{E}_{0}\right|^{2}} \\
& =\frac{2 \pi}{\lambda \varepsilon_{0} \sqrt{\varepsilon_{\mathrm{m}}}} L_{\mathrm{m}}^{2} \frac{\left|\hat{\mathbf{e}}_{\mathrm{d}} \cdot \mathbf{E}_{\mathrm{loc}}\right|^{2}}{\left|\mathbf{E}_{0}\right|^{2}} \Im\left(\alpha_{z z}\right) .
\end{aligned}
$$


For a bare dye, its absorption cross-section $\sigma_{\mathrm{Abs}}^{0}$ is given by:

$$
\sigma_{\mathrm{Abs}}^{0}=\frac{2 \pi}{\lambda \varepsilon_{0} \sqrt{\varepsilon_{\mathrm{m}}}} L_{\mathrm{m}}^{2} \frac{\Im\left(\alpha_{z z}\right)}{3} .
$$

So, we can rewrite Eq. (3.62) as:

$$
\sigma_{\mathrm{Abs}}=\sigma_{\mathrm{Abs}}^{0} \frac{3\left|\hat{\mathbf{e}}_{\mathrm{d}} \cdot \mathbf{E}_{\mathrm{loc}}\right|^{2}}{\left|\mathbf{E}_{0}\right|^{2}}
$$

The absorption cross-section above is for a single dye molecule, but can be extended to a collection of $N$ randomly adsorbed molecules by considering the surface-average of the local field. For simplicity, we will restrict ourselves to consider only tree types of adsorption orientations (as depicted in Fig. 3.2), which are random orientation $(*)$, perpendicular to the surface $(\perp)$, and randomly oriented orientation parallel to the surface $(\|)$. In all three cases, the absorption cross-section for $N$ molecules can be expressed as:

$$
C_{\mathrm{Abs}}=N \sigma_{\mathrm{Abs}}^{0} M(\lambda)
$$

where, $M$ is the absorption enhancement factor and is wavelength dependent. For each case, it is given as:

$$
\begin{aligned}
M^{*}(\lambda) & =\frac{\left\langle\left|\mathbf{E}_{\mathrm{loc}}\right|^{2}\right\rangle}{\left|\mathbf{E}_{0}\right|^{2}} \\
M^{\perp}(\lambda) & =\frac{3\left\langle\left|\mathbf{E}_{\mathrm{loc}}^{\perp}\right|^{2}\right\rangle}{\left|\mathbf{E}_{0}\right|^{2}} \\
M^{\|}(\lambda) & =\frac{3\left\langle\left|\mathbf{E}_{\mathrm{loc}}^{\|}\right|^{2}\right\rangle}{2\left|\mathbf{E}_{0}\right|^{2}},
\end{aligned}
$$

where $\langle$.$\rangle represents the averaging over the sphere's surface (since the local fields of$ the molecules are not uniform). By doing the averaging over the possible orientations of the dye molecules, it leads to an additional factor of $1 / 3$ for $(*), 1$ for $(\perp)$, and $1 / 2$ for $(\|)$ which are then multiplied by the existing factor of $3 \mathrm{in} \mathrm{Eq.} \mathrm{3.64.} \mathrm{The}$ orientation averaging can be done as follow: for example, in the $(*)$ case, we can start by writing $\mathbf{E}_{\mathrm{loc}}$ and $\hat{\mathbf{e}}_{\mathrm{d}}$ as:

$$
\begin{aligned}
\mathbf{E}_{\mathrm{loc}} & =\mathbf{E}_{\mathrm{loc}}^{\perp}+\mathbf{E}_{\mathrm{loc}}^{\|} \\
& =\mathrm{E}_{\mathrm{loc}, \mathrm{r}} \hat{\mathbf{r}}+\mathrm{E}_{\mathrm{loc}, \theta} \hat{\theta}+\mathrm{E}_{\mathrm{loc}, \phi} \hat{\phi} \\
\hat{\mathbf{e}}_{\mathrm{d}} & =\cos \theta \hat{\mathbf{r}}+\sin \theta \cos \phi \hat{\theta}+\sin \theta \sin \phi \hat{\phi} .
\end{aligned}
$$


From these, we can calculate the orientation averaging as:

$$
\begin{aligned}
& \frac{1}{2 \pi} \iint\left|\hat{\mathbf{e}}_{\mathrm{d}} \cdot \mathrm{E}_{\mathrm{loc}}\right|^{2} \sin \theta d \theta d \phi \\
& \quad=\frac{1}{2 \pi} \int_{0}^{2 \pi} \int_{0}^{\pi / 2}\left|\mathrm{E}_{\mathrm{loc}, \mathrm{r}} \cos \theta+\mathrm{E}_{\mathrm{loc}, \theta} \sin \theta \cos \phi+\mathrm{E}_{\mathrm{loc}, \phi} \sin \theta \sin \phi\right|^{2} \sin \theta d \theta d \phi \\
& \quad=\frac{1}{2 \pi} \times \frac{2 \pi}{3}\left(\left|\mathrm{E}_{\mathrm{loc}, \mathrm{r}}\right|^{2}+\left|\mathrm{E}_{\mathrm{loc}, \theta}\right|^{2}+\left|\mathrm{E}_{\mathrm{loc}, \phi}\right|^{2}\right)=\frac{1}{3}\left|\mathrm{E}_{\mathrm{loc}}\right|^{2}
\end{aligned}
$$

For the other two cases, a similar procedure can easily be followed to get the factor of 1 and $1 / 2$ for $\perp$ and $\|$, respectively.

At a low concentration of dye-molecules, it is more practical and useful to look at the differential absorption cross-section $\left(\delta \sigma_{\mathrm{Abs}}\right)$ as the absorption cross-section of the dyes would be insignificant compared to that of the metallic nanosphere. To do so, one can measure the bare sphere separately and subtract it from the coated sphere spectrum to observe $\delta \sigma_{\mathrm{Abs}}$ as explained in Ref. [34]. Within the approximation of the microscopic model, the differential cross-section spectrum is then given by $C_{\mathrm{Abs}}$, as expressed in Eq. 3.65. This microscopic model, however, does not account for neither the possible effect of the dye molecules onto the particle responses nor the local dye-dye interactions.

\subsubsection{Effective Medium Model (Effective Dielectric Function)}

This model replaces the discrete dye layer of given coverage $\rho$ (no. dye $/ \mathrm{nm}^{2}$ ) by a homogeneous shell of thickness $d$ with volume $V_{s}$ and the volumic dye concentration $c_{d}=N / V_{s} \approx \rho / l_{\mathrm{s}}$ (no. dye $/ \mathrm{nm}^{3}$ ). The Clausius-Mossotti relation (also known as Lorentz-Lorenz relation) is an effective medium model which is commonly used to relate the polarisability $\left(\alpha_{d}\right)$ of the molecules to its dielectric function $(\varepsilon)$ for isotropic media [62, 63]. Usually, if one of these quantities is known or given, the other one can be obtained via the CM relation, which is:

$$
\bar{\alpha}_{d}=\frac{c_{d} \alpha_{d}}{3 \varepsilon_{0}}=\frac{\varepsilon-1}{\varepsilon+2} \quad \quad \text { or } \quad \varepsilon=\frac{1+2 \bar{\alpha}_{d}}{1-\bar{\alpha}_{d}} .
$$

The CM relation works well at a low concentration of the dye molecules (dilute regime) for isotropic media (no orientation effects) but cannot be applied to anisotropic media. Therefore, generalising this effective medium approximation from a 3D isotropic to a 2D anisotropic medium is in great need in this project and will be discussed more in the next chapter. For the time being, we will neglect them here and only consider a much simpler dilute limit. For a homogeneous shell embedded 
in a medium with dielectric constant $\varepsilon_{\mathrm{m}}$, the effective dielectric function for the case of fully random orientation of the dye molecules can then be defined as:

$$
\varepsilon_{\mathrm{eff}}=\frac{1+2\left(\bar{\alpha}_{d}+\bar{\alpha}_{\mathrm{m}}\right)}{1-\left(\bar{\alpha}_{d}+\bar{\alpha}_{\mathrm{m}}\right)}
$$

In the dilute case, this can be reduced to:

$$
\begin{gathered}
\varepsilon^{*}=\varepsilon_{\mathrm{eff}}=\varepsilon_{\mathrm{m}}+L_{\mathrm{m}}^{2} \frac{c_{d} \alpha_{\mathrm{zz}}}{3 \varepsilon_{0}} \\
\text { with, } \quad \alpha_{\mathrm{zz}}(\lambda)=3 \alpha_{d}(\lambda) .
\end{gathered}
$$

We may also use the microscopic model Eqs. 3.67 and 3.68 as a guide to generalise Eq. 3.74 to the anisotropic cases. This leads to, for radially oriented molecules:

$$
\begin{aligned}
\varepsilon_{\mathrm{n}}^{\perp} & =\varepsilon_{\mathrm{m}}+L_{\mathrm{m}}^{2} \frac{c_{d} \alpha_{\mathrm{zz}}}{\varepsilon_{0}} \\
\varepsilon_{\mathrm{t}}^{\perp} & =\varepsilon_{\mathrm{m}} .
\end{aligned}
$$

For randomly oriented orientation parallel to the surface, we have:

$$
\begin{aligned}
\varepsilon_{\mathrm{n}}^{\|} & =\varepsilon_{\mathrm{m}} \\
\varepsilon_{\mathrm{t}}^{\|} & =\varepsilon_{\mathrm{m}}+L_{\mathrm{m}}^{2} \frac{c_{d} \alpha_{\mathrm{zz}}}{2 \varepsilon_{0}} .
\end{aligned}
$$

\subsubsection{Comparison Between Full Anisotropic Mie and TASA}

To check the validity of our TASA model, we will need to look at its prediction of the optical properties of a dye-coated nanosphere and compare to those from different models presented earlier: full anisotropic Mie theory by Roth and Dignam (RD) ${ }^{1}$ and microscopic model (MM). For this comparison, we choose to focus on a $30 \mathrm{~nm}$, radius silver sphere with a dielectric function, as given in Ref. [27] and the embedding medium is chosen a water $\varepsilon_{\mathrm{m}}=1.33^{2}$ to be more suitable to experiments. The shell thickness is set to be $l_{\mathrm{s}}=0.3 \mathrm{~nm}$ for the TASA calculations. For the dye polarisability [65], we use:

$$
\alpha_{\mathrm{zz}}=\alpha_{\mathrm{uni}}=\alpha_{\infty}-\frac{\alpha_{1} \lambda_{1}}{\mu_{1}}\left[1-\frac{1}{1-\frac{\lambda_{1}^{2}}{\lambda^{2}}-i \frac{\lambda_{1}^{2}}{\lambda \mu_{1}}}\right],
$$

with $\alpha_{\infty}=9.4 \times 10^{-39}[\mathrm{SI}], \alpha_{1}=5.76 \times 10^{-38}[\mathrm{SI}], \lambda_{1}=526 \mathrm{~nm}$, and $\mu_{1}=10000 \mathrm{~nm}$. This is a simple Lorentz oscillator model with parameters matching the experimentally measured optical properties of the main absorption peak of Rhodamine 6G in

\footnotetext{
${ }^{1}$ Our preferred simulation programme, Matlab, was used and all the codes can be found from SPlaC codes [64], with additional routines developed based on Lanzcos series approximations to calculate the complex Gamma functions, see Appendix A for more details.
} 

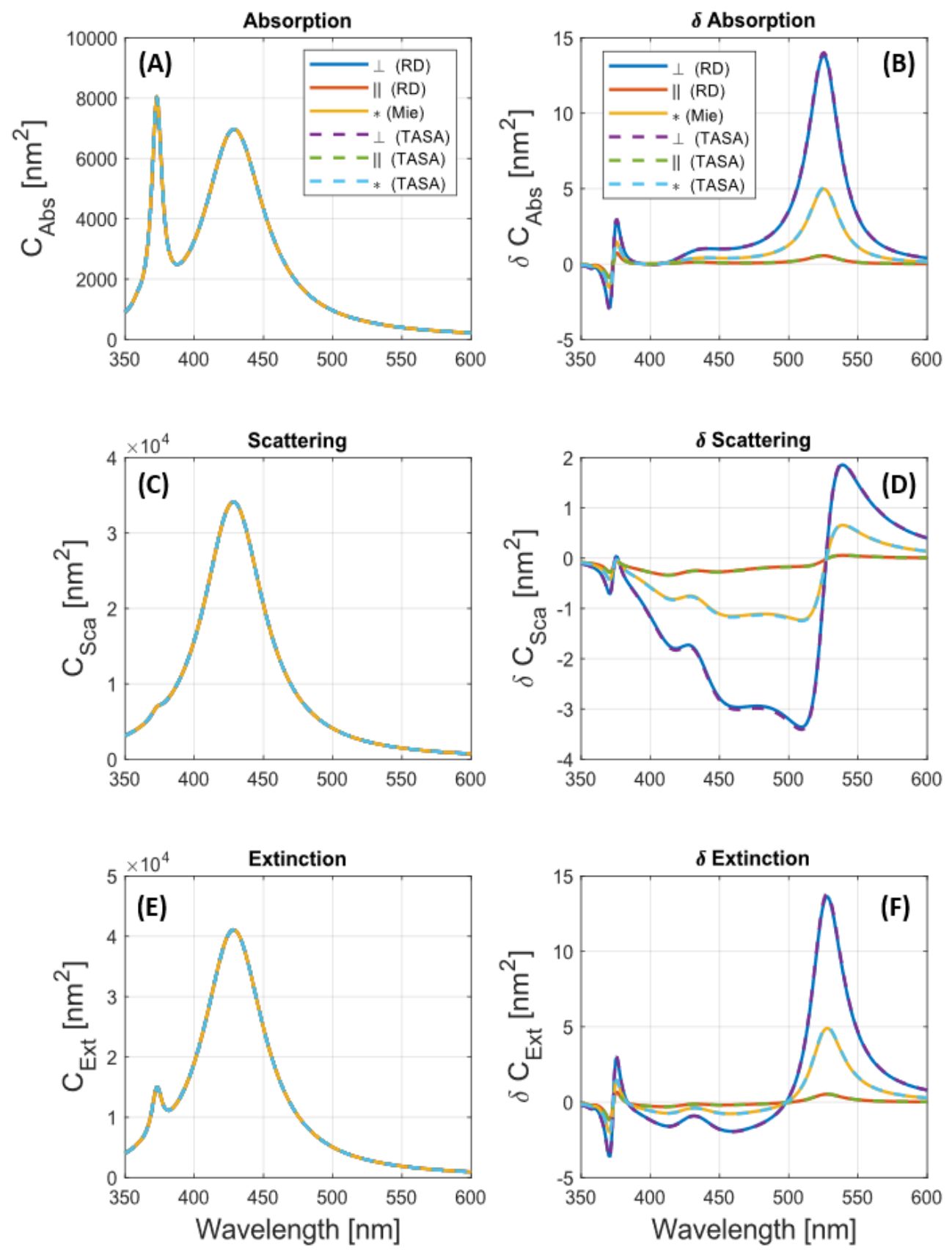

Figure 3.3: Predictions of the optical properties for a silver sphere of radius $a=30 \mathrm{~nm}$ in water $\varepsilon_{\mathrm{m}}=1.33^{2}$, coated with a dye layer of low coverage $\rho=0.001 \mathrm{~nm}^{2}$ with its thickness of $l_{s}=0.3 \mathrm{~nm}$ and polarisability $\alpha_{z z}$. In the two anisotropic cases, we compare the results from full anisotropic Mie theory by Roth and Dignam (RD) with our thin shell approximation (TASA). The left column shows the absorption (A), scattering (C), and extinction (E) cross-sections, which are indistinguishable in all six cases, as the response of the silver sphere is most dominant. We, therefore, show in the right column $(\mathrm{B}, \mathrm{D}, \mathrm{F})$ the corresponding differential cross-sections with the bare sphere response subtracted. 
water, where the local field correction factor is accounted for (the same notations as in Ref. [35]).

We will now use our TASA model to predict the optical properties of the dyecoated silver nanosphere (absorption, scattering, and extinction cross-sections and their corresponding differential cross-section) and compare them to those obtained by RD shown in Fig. 3.3. For the absorption, scattering, and extinction crosssections of the system (core and shell, shown in A, C, and E, respectively), the results do not reveal clearly the shell properties as they are strongly dominated by the silver sphere response. Therefore, we focus on their corresponding differential crosssections (B, D and F), where we subtracted the silver sphere response (calculated using Mie theory) from the total cross-sections of the whole system (shown on the left column of Fig. 3.3). The results showing the differential cross-section on the right column of Fig. 3.3 are barely distinguishable in all the cases, except the region of zero-crossing, and the relative error between the two models is in the order of $2 \%$ for $*$ and $\perp$, and $5 \%$ for the $\|$ case. From these results, we can safely conclude that our TASA model worked and agreed with the Full anisotropic Mie theory at the limit of small $l_{s} \ll a, b$.

\subsection{Summary and Outlook}

We have simplified the full solutions of anisotropic Mie theory (by Roth and Dignam [40]) by assuming the thin shell approximation to the first order of Taylor's expansion. The full solutions of the problem require calculations of Bessel functions of complex order, which are not built-in in many standard computing software (Matlab, for example) and, for this reason, it may have limited this theoretical approach to model relevant problems and experiments. However, these complex ordered Bessel functions can be calculated with newly developed routines in SPlaC codes in Ref. [64]. We then derived the thin anisotropic shell approximation to the first approximation, as done early and the results were published in Ref. [48]. Our TASA model obtained simple approximation for the Mie scattering coefficients, which no longer require the computations of complex ordered Bessel functions. The approximation is valid in the limit of thin shells (with thickness $l_{\mathrm{s}}=b-a \ll a$ ). With adequate comparisons (not shown here), we are able to conclude that the results from the full anisotropic Mie theory and TASA agreed very well for the shell thickness of the order of $1 \mathrm{~nm}$ or less with the core radius of $30 \mathrm{~nm}$, (i.e. 1:30 shell to radius ratio). 

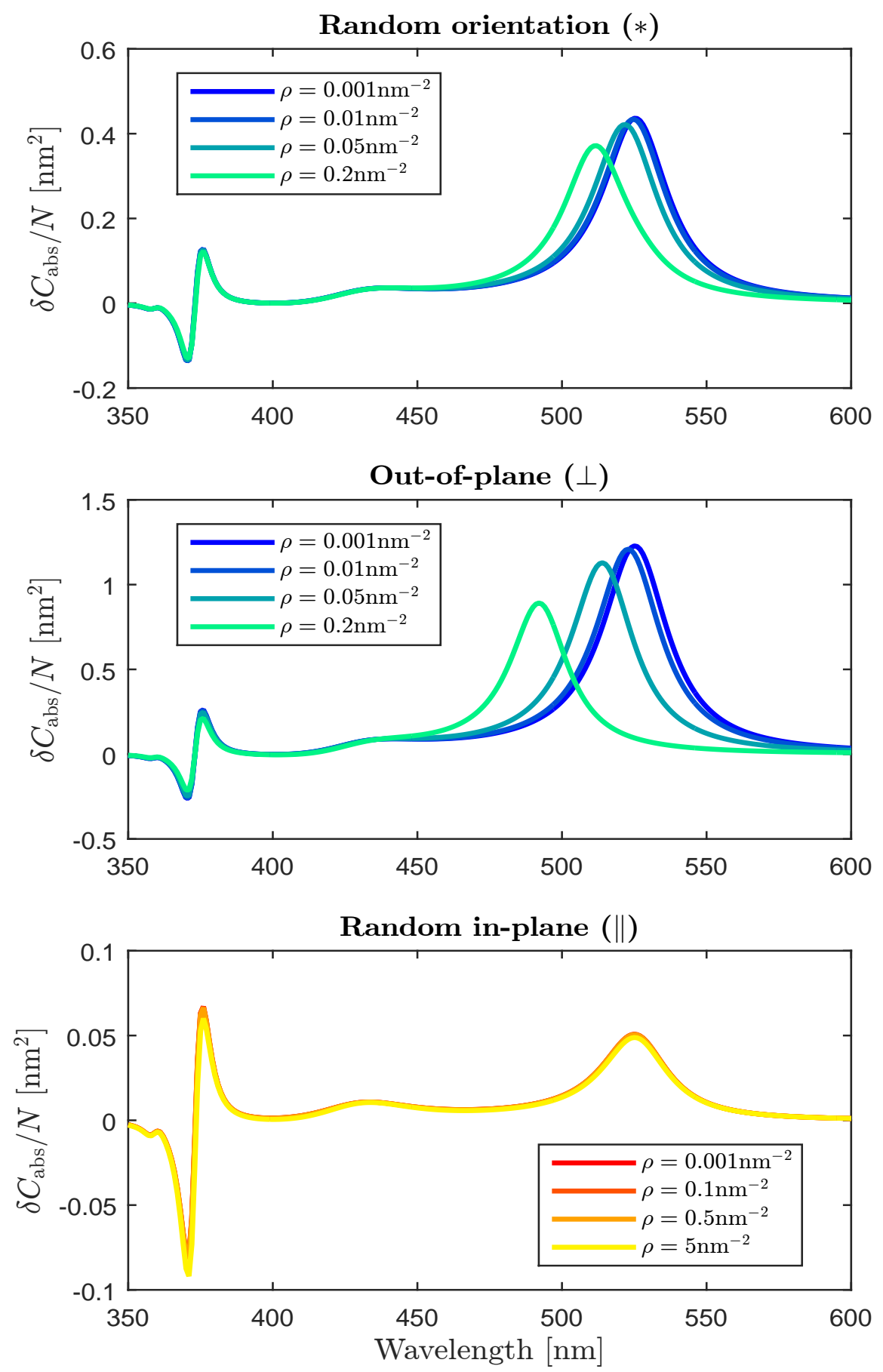

Figure 3.4: The differential absorption cross-sections for the 3 different orientations $*, \perp$, and || predicted by anisotropic Mie theory (within TASA) showing dye-coverage dependence. 
In this chapter, we used the dielectric functions from the effective medium shell model for random orientation case and used the microscopic model as a guide to generalise that to the other two cases $(\perp$ and $\|)$, in the dilute regime. The choice of these dielectric functions for the shell worked surprisingly well for the low dye-coverage concentration $\rho$. The field enhancement depends strongly on the orientation of the molecules. The results can be shown (even at a very low dye concentration) that the enhancement for the $\perp$ orientation is much higher than the $\|$ orientation case $\left(M^{\perp} \sim 25\right.$ times stronger than $\left.M^{\|}\right)$. This is useful evidence to show that the orientation effect of the molecules adsorbed onto metallic surfaces is significantly important and, therefore, must be taken into account for those molecules that have a preferred adsorbed orientation, for examples, $\mathrm{J}$ and H-aggregates [31, 66, 67, 68].

The results of the coverage-dependence are shown in Fig. 3.4, which depend on the dye orientation: a blue-shift for $*$, more pronounced blue-shift for $\perp$, and no shift for $\|$, even at higher concentration. For the radial orientation $\perp$, the shifts ate similar to those from the dye-dye interactions and are expected at high concentration, as evidenced in Ref. [35]. Surprisingly, the red-shift that is expected for the $\|$ orientation case was not observed. This is due to the fact that the dilute effective medium shell model does not include the local field corrections (dye-dye interactions), which are important at high concentration. So, the extension to this will be to incorporate the local field effect relating to the CM relation correction into the anisotropic Mie model for the shell, which will be carried out in Chapter 4 .

The anisotropic effective shell model shows evidence for the shift in the plasmon resonances of the silver sphere (especially at the quadrupolar resonance), which is not captured by the microscopic model. The shifts in the plasmon resonances are expected and have been investigated in Ref [25], but how dye-orientation may affect these shifts has not been discussed. Therefore, in chapter 6 , we will investigate in further details how the dye-orientation and their locations of the adsorption onto the surface of the sphere may affect the shifts in the plasmon resonance. 


\section{Refined Model for Effective Dielectric Func- tions in Anisotropic Media}

In the previous chapter, we have derived the scattering coefficients, as well as the internal coefficients, of the light scattering problem by an isotropic core/anisotropic shell system. The dielectric tensor used for the anisotropic shell was derived from the microscopic approach of a single dye-molecules which works fairly good in the dilute cases. However, these dielectric functions did not account for the local field corrections due to the dye-dye interactions, which are important at high concentration of the dye molecules. The other important part of determining the dielectric functions for the anisotropic shell, which is usually ignored, is the image dipole effect $[45,46]$. The image dipole or self-reflected fields will only become dominant when the separation distance between the surface of the nanoparticle and the molecule position is small, otherwise, this effect can be safely neglected. The main goal of this chapter is, therefore, to obtain the relative dielectric functions for the anisotropic shell, $\varepsilon_{\mathrm{n}}$ and $\varepsilon_{\mathrm{t}}$, where the dye-dye interactions and the image-dipole effect are correctly taken into account to reproduce compatible results from those of the GCDM. To do so, we will combine and extend several concepts that have been developed previously in isolation into a refined effective medium model.

Dignam and Moskovits [44] had derived the generalisation of the effective dielectric functions to a $2 \mathrm{D}$ anisotropic medium in the context of a $2 \mathrm{D}$ planar array of dipoles and this work was further developed to include the image-dipole effects by Bagchi et al. [45]. These previous works have derived the effective dielectric functions, which include the dye-dye interactions and the image-dipole effects. However, they were carried out for dipoles/molecules in vacuum. Therefore, the generalisation of the 2D anisotropic effective dielectric functions for molecules in an embedding medium with permittivity $\varepsilon_{\mathrm{m}}$ is needed to make our theoretical predictions relevant to many experimental implementations in water or other solvents. In this chapter, we will go through the derivations of the effective dielectric functions for a $2 \mathrm{D}$ 
anisotropic medium, implement the results of these dielectric functions into appropriate theory i.e. anisotropic Mie (anisotropic Fresnel equations), then compare against GCDM (an improved microscopic model for a 2D layer of dye molecules) to check their validity.

\subsection{The Dielectric Function(DF) for Molecular Monolayer}

Among all the related previous studies, most of them have constructed $\varepsilon$ for the dye layer empirically based on accounting the molecular resonance(s) using one (or more) Lorentz oscillator(s). This method, however, cannot relate the dielectric function of the dye layer $(\varepsilon)$ to its surface coverage $(\rho)$. Therefore, in the following sections, we are going through a more rigorous approach to express the dielectric functions $\varepsilon$ in terms of the microscopic properties, i.e. the polarisability $(\alpha)$, surface concentration $(\rho)$. We will go through several approaches and discuss which one is most suited for our model of interest.

\subsubsection{Isotropic DF for 3D bulk}

For now, we will consider the isotropic case where randomly oriented molecules are distributed uniformly and embedded in a medium with permittivity $\varepsilon_{\mathrm{m}}$. In doing so, we shall start with the standard Clausius Mossotti relation.

\section{A. Standard Clausius-Mossotti}

As briefly mentioned in the previous chapter (section. 2.2.2), if we consider a 3D collection of water molecules with polarisability $\alpha_{\mathrm{m}}$ to be in an external field $\mathbf{E}_{\text {Ext }}$, then the dipole moment of $\mathrm{i}$-th molecule is:

$$
\mathbf{p}_{\mathrm{i}}=\alpha_{\mathrm{m}}\left[\mathbf{E}_{\mathrm{Ext}}+\sum_{\mathrm{j} \neq \mathrm{i}} \mathbf{E}_{\text {dip }}\left(\mathbf{p}_{\mathrm{j}}, \mathbf{r}_{\mathrm{i}}-\mathbf{r}_{\mathrm{j}}\right)\right],
$$

where $\mathbf{E}_{\text {dip }}\left(\mathbf{p}_{\mathrm{j}}, \mathbf{r}_{\mathrm{i}}-\mathbf{r}_{\mathrm{j}}\right)$ is the electric field at the dipole position due to another dipole $\mathbf{p}_{\mathrm{j}}$ located at $\mathbf{r}_{\mathrm{j}}$. It is given as:

$$
\mathbf{E}_{\text {dip }}(\mathbf{p}, \mathbf{r})=-\nabla\left(\frac{\mathbf{p} \cdot \hat{\mathbf{r}}}{4 \pi \varepsilon_{0} \mathrm{r}^{2}}\right)=\frac{3(\mathbf{p} \cdot \hat{\mathbf{r}}) \hat{\mathbf{r}}-\mathbf{p}}{4 \pi \varepsilon_{0} \mathrm{r}^{3}}
$$

where $\overrightarrow{\mathbf{r}}_{i}=\left(x_{i}, y_{i}\right), \mathrm{r}_{i}=\left(x_{i}^{2}+y_{i}^{2}\right)^{1 / 2}$, and $\hat{\mathbf{r}}_{i}=\overrightarrow{\mathbf{r}}_{i} / \mathrm{r}_{i}$. The second term of Eq. 4.1 can then be expressed in terms of the volumic concentration of the dipoles $c_{\mathrm{m}}$ and the 
lattice sum $\xi_{m}$ (which can be found as $\xi_{\mathrm{m}}=4 \pi / 3$ for a $3 \mathrm{D}$ cubic lattice). Equation 4.1 can then be re-expressed as (averaging over all the dipoles):

$$
\mathbf{p}_{\mathrm{i}}=\frac{\alpha_{\mathrm{m}}}{1-c_{\mathrm{m}} \alpha_{\mathrm{m}} /\left(3 \varepsilon_{0}\right)} \mathbf{E}_{\mathrm{Ext}} .
$$

From the macroscopic definition, the dipole moment per unit volume (polarization) is defined as:

$$
\mathbf{P}=c_{\mathrm{m}} \mathbf{p}_{i}=\frac{3 \varepsilon_{0} \bar{\alpha}_{\mathrm{m}}}{1-\bar{\alpha}_{\mathrm{m}}} \mathbf{E}_{\mathrm{Ext}}
$$

where,

$$
\bar{\alpha}_{\mathrm{m}}=\frac{c_{\mathrm{m}} \alpha_{\mathrm{m}}}{3 \varepsilon_{0}} .
$$

If $\mathbf{E}_{\mathrm{Ext}}$ is identified with the macroscopic field, then we can make use of the macroscopic definition of the electric displacement $\mathbf{P}=\varepsilon_{0}\left(\varepsilon_{\mathrm{m}}-1\right) \mathbf{E}_{\mathrm{Ext}}$, which leads to the $\mathrm{CM}$ relation as:

$$
\varepsilon_{\mathrm{m}}=1+\frac{3 \bar{\alpha}_{\mathrm{m}}}{1-\bar{\alpha}_{\mathrm{m}}}=\frac{1+2 \bar{\alpha}_{\mathrm{m}}}{1-\bar{\alpha}_{\mathrm{m}}}
$$

This is exactly the same expression shown in Eq. 3.72 .

\section{B. Dye-molecules in water (3D) - microscopic approach}

Now we add dye molecules and find the effective dielectric function $\varepsilon_{\text {eff }}$ of the dyewater combination. The procedure of this approach is the same as above with the additional contributions of dye-molecules (or dye dipoles). In doing so, we get:

$$
\begin{aligned}
\varepsilon_{\mathrm{eff}} & =\frac{1+2 \bar{\alpha}_{\mathrm{m}}+2 \bar{\alpha}_{\mathrm{d}}}{1-\bar{\alpha}_{\mathrm{m}}-\bar{\alpha}_{\mathrm{d}}} \\
& =\varepsilon_{\mathrm{m}}+\frac{3 L_{\mathrm{m}}^{2} \bar{\alpha}_{\mathrm{d}}}{1-L_{\mathrm{m}} \bar{\alpha}_{\mathrm{d}}} .
\end{aligned}
$$

For the low dye concentration limit (small $\rho$ ), this will reduce to the same expression as presented in Eq. 3.74, which is:

$$
\varepsilon_{\mathrm{eff}} \approx \varepsilon_{\mathrm{m}}+3 L_{\mathrm{m}}^{2} \bar{\alpha}_{\mathrm{d}}
$$

\section{Dye-molecules in water (3D) - continuum approach}

This is a hybrid approach where we treat the dye molecules as discrete point dipoles embedded in a continuous dielectric medium with $\varepsilon_{\mathrm{m}}$. The external field is a macroscopic field. Hence we can write the dipole moment of the dye molecule as:

$$
\begin{aligned}
\mathbf{p}_{\mathrm{d}} & =\alpha_{\mathrm{d}} \mathbf{e}_{\mathrm{loc}}=\alpha_{\mathrm{d}} L_{\mathrm{m}} \mathbf{E}_{\mathrm{loc}} \\
& =\alpha_{\mathrm{d}} L_{\mathrm{m}}\left[\mathbf{E}_{\mathrm{Ext}}+L_{\mathrm{m}} \frac{\mathbf{p}_{\mathrm{d}}}{4 \pi \varepsilon_{0} \varepsilon_{\mathrm{m}}} c_{\mathrm{d}} \xi_{\mathrm{d}}\right] .
\end{aligned}
$$


The $L_{\mathrm{m}}$ factor inside the bracket is justified by reciprocity. One can also consider the fact that the spontaneous emission rate for an emitter in a medium scales as $L_{\mathrm{m}}^{2}$, so another $L_{\mathrm{m}}$ factor is needed to ensure this is satisfied. Also if the dye molecules arranged in a $3 \mathrm{D}$ cubic lattice, then $\xi_{\mathrm{d}}=\xi_{\mathrm{m}}=4 \pi / 3$. Therefore, we have:

$$
\mathbf{p}_{\mathrm{d}}=\frac{\alpha_{\mathrm{d}} L_{\mathrm{m}}}{1-\bar{\alpha}_{\mathrm{d}} L_{\mathrm{m}}^{2} / \varepsilon_{\mathrm{m}}} \mathbf{E}_{\mathrm{Ext}}
$$

We now may write the macroscopic polarisation as:

$$
\begin{aligned}
& \quad \mathbf{P}=\varepsilon_{0}\left(\varepsilon_{\mathrm{eff}}-1\right) \mathbf{E}_{\mathrm{Ext}} \\
& =\mathbf{P}_{\mathrm{d}}+\mathbf{P}_{\mathrm{m}}=L_{\mathrm{m}} c_{\mathrm{d}} \mathbf{p}_{\mathrm{d}}+\varepsilon_{0}\left(\varepsilon_{\mathrm{m}}-1\right) \mathbf{E}_{\mathrm{Ext}} \\
& \text { hence, } \quad \varepsilon_{\mathrm{eff}}=\varepsilon_{\mathrm{m}}+\frac{3 \bar{\alpha}_{\mathrm{d}} L_{\mathrm{m}}^{2}}{1-\bar{\alpha}_{\mathrm{d}} L_{\mathrm{m}}^{2} / \varepsilon_{\mathrm{m}}} .
\end{aligned}
$$

In the dilute case, this will be simplified to the same expression as Eq. 3.74.

\section{Microscopic Approach versus Continuum Approach}

Although the effective dielectric function converges to the same expression for both models at low concentration of dye molecules, there are still some discrepancies in $\varepsilon_{\text {eff }}$ (as shown in Eqs. 4.6 and 4.12) between the two models at high concentration of dye molecules. These two models should work in different situations as we have treated the water molecules differently. In the microscopic approach, it should work well if the number of dye molecules is comparable to those of water (i.e. $c_{\mathrm{d}} \approx c_{\mathrm{m}}$ ). If there are many more water molecules than dye-molecules (i.e. $c_{\mathrm{d}} \ll c_{\mathrm{m}}$ ) then all the water molecules should be treated as a continuous medium with dielectric function $\varepsilon_{\mathrm{m}}$, as done in the continuum approach.

This isotropic effective medium model (EMM) has been used in many previous studies $[69,34]$, but it fails to account for the effect of molecules' orientations on their optical response, their mutual interactions, and the image dipole effect. The adsorption orientation of the molecules adsorbed on metallic nanoparticles can significantly affect the optical response of the system, as evidenced in the context of surface selection rules in SERS, [27, 61, 59, 60]. The image dipole effects cannot be neglected when molecules are close to a metal surface [37]. These shortcomings can be overcome by applying the concepts of previous studies of adsorbed monolayers on flat surfaces in the context of ellipsometry $[44,70,71]$ or differential reflectance spectroscopy $[45,46]$. 
(A) Isotropic in-plane

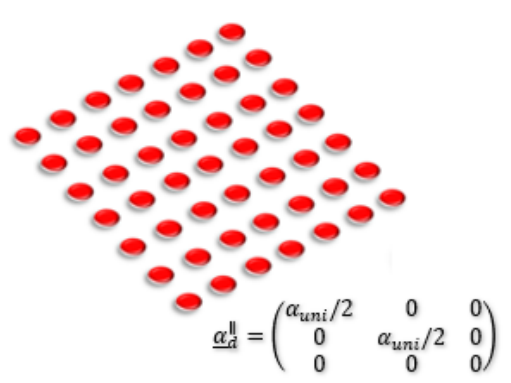

(B) Perpendicular

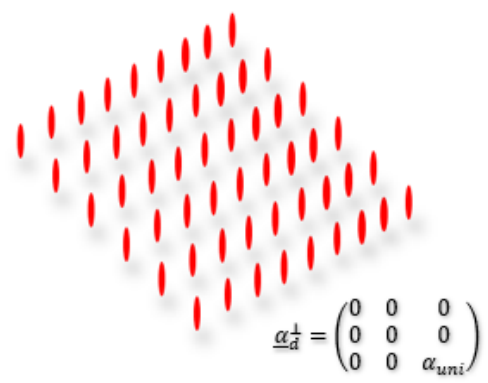

Figure 4.1: The two orientations of uni-axial dye molecules of our interests: (A) isotropically in-plane (smarties like dipoles $\|$ ) and (B) perpendicular (or radially oriented dipoles, $\perp)$. In both cases, we consider the dyes to be arranged in square lattice.

\subsubsection{Anisotropic DF for 2D Planar Array of Dipoles in Vacuum}

In the first half of this section, we will follow previous work [44] to determine the dielectric functions for the anisotropic shell in our problem, by modelling the dye molecules as an infinite planar array of dipoles in two-dimensional space (microscopic model), as depicted in Fig. 4.1, to capture the dye-dye interactions for different orientations of the dye molecules.

To do so, we will consider a 2-D infinite planar array of dipoles (in vacuum) arranged in a square lattice (all with the same polarisability $\alpha_{\mathrm{d}}$ and positioned in the $x y$-plane). For a given external incident field $\mathbf{E}_{\text {Ext }}$, the local field at the dye position, $\mathbf{E}_{\text {loc }}$ can be computed analytically and it induces an oscillating dipole moment $\mathbf{p}=\underline{\alpha}_{\mathrm{d}} \mathbf{E}_{\text {loc }}$. Taking into account dipole-dipole interactions, the dipole moment $\mathbf{p}$ for a dipole located at the origin $\mathbf{0}$ is given by:

$$
\mathbf{p}(\mathbf{0})=\underline{\alpha}_{\mathrm{d}}\left[\mathbf{E}_{\mathrm{Ext}}+\sum_{\mathbf{r}_{n} \neq \mathbf{0}} \mathbf{E}_{\mathrm{dip}}\left(\mathbf{p}, \mathbf{r}_{n}\right)\right]
$$

We here consider two different cases in the dye-molecules orientation, as depicted in Fig. 4.1, which is relevant to experiments. These happen when the molecular axes are tangential to the surface (i.e., the in-plane excitation, figure. 4.1A, for smarties-like dipoles) and perpendicular to the surface (i.e., out-of-plane excitation, figure. 4.1B). 


\section{A. Dipoles pointing in $\hat{\mathrm{x}}-$ or $\hat{\mathrm{y}}$ - direction}

If all of the dipoles are pointing in the $\hat{\mathbf{x}}$-direction, then the total $\hat{\mathbf{y}}$-component of $\mathbf{E}_{\text {dip }}$ will be cancelled out exactly (as they are arranged accordingly to the square lattice in the $x y$-plane), and there will be no $\hat{\mathbf{z}}$-component either. The dipole moment in the $\hat{\mathbf{x}}$-direction is:

$$
\begin{aligned}
\mathrm{p}_{x} & =\alpha\left[\mathrm{E}_{\mathrm{Ext}, x}+\frac{1}{4 \pi \varepsilon_{0}} \mathrm{p}_{x} \sum_{\mathbf{r}_{n} \neq \mathbf{0}} \frac{2 x_{n}^{2}-y_{n}^{2}}{\left(x_{n}^{2}+y_{n}^{2}\right)^{5 / 2}}\right] \\
& =\frac{\alpha}{1-\frac{\alpha}{4 \pi \varepsilon_{0}} \sum_{\mathbf{r}_{n} \neq \mathbf{0}} \frac{2 x_{n}^{2}-y_{n}^{2}}{\left(x_{n}^{2}+y_{n}^{2}\right)^{5 / 2}}} \mathrm{E}_{\mathrm{Ext}, x} .
\end{aligned}
$$

We then can make use of the macroscopic definition of the electric displacement $\mathrm{P}_{x}=c_{\mathrm{d}} \mathrm{p}_{x}=\varepsilon_{0}\left(\varepsilon_{x x}-1\right) \mathrm{E}_{\text {Macro, } x}$ with the boundary condition, at which the tangential component of the electric field is continuous across a boundary, i.e. $\mathrm{E}_{\mathrm{Ext}, x}=$ $\mathrm{E}_{\text {Macro, } x}$. This leads to:

$$
\varepsilon_{x x}=1+\frac{c_{\mathrm{d}} \alpha / \varepsilon_{0}}{1-\frac{\alpha}{4 \pi \varepsilon_{0}} \sum_{\mathbf{r}_{n} \neq \mathbf{0}} \frac{2 x_{n}^{2}-y_{n}^{2}}{\left(x_{n}^{2}+y_{n}^{2}\right)^{5 / 2}}} .
$$

Similarly, if all dipoles point in $\hat{\mathbf{y}}$-direction, then the $\hat{\mathbf{x}}$-component of $\mathbf{E}_{\text {dip }}$ will be cancelled exactly and the above derivations can be reproduced to get:

$$
\varepsilon_{y y}=1+\frac{c_{\mathrm{d}} \alpha / \varepsilon_{0}}{1-\frac{\alpha}{4 \pi \varepsilon_{0}} \sum_{\mathbf{r}_{n} \neq \mathbf{0}} \frac{2 y_{n}^{2}-x_{n}^{2}}{\left(x_{n}^{2}+y_{n}^{2}\right)^{5 / 2}}} .
$$

As the dipoles are arranged in a square lattice, the lattice sum in the Eqs. 4.15 and 4.16 can be calculated as (in Ref. [71]):

$$
\begin{aligned}
\sum_{\mathbf{r}_{n} \neq \mathbf{0}} \frac{2 x_{n}^{2}-y_{n}^{2}}{\left(x_{n}^{2}+y_{n}^{2}\right)^{5 / 2}} & =\sum_{\mathbf{r}_{n} \neq \mathbf{0}} \frac{2 y_{n}^{2}-x_{n}^{2}}{\left(x_{n}^{2}+y_{n}^{2}\right)^{5 / 2}}=\frac{1}{2} \sum_{\mathbf{r}_{n} \neq \mathbf{0}} \frac{1}{\left(x_{n}^{2}+y_{n}^{2}\right)^{3 / 2}} \\
& =\rho^{3 / 2} \xi_{0} / 2 \approx \rho^{3 / 2}\left[4 \zeta(3)-32 \pi^{2} K_{0}(2 \pi)\right] .
\end{aligned}
$$

Therefore, Eqs. 4.15 and 4.16 can be rewritten as:

$$
\varepsilon_{x x}=\varepsilon_{y y}=1+\frac{c_{\mathrm{d}} \alpha / \varepsilon_{0}}{1-\frac{\alpha}{4 \pi \varepsilon_{0}} \rho^{3 / 2} \xi_{0} / 2},
$$

where $\alpha=\alpha_{\text {uni. }}$. If the dye molecules are smarties-like dipoles (see Fig. $4.1 \mathrm{~A}$ ),

$$
\varepsilon_{x y}=1+\frac{c_{\mathrm{d}} \alpha / \varepsilon_{0}}{1-\frac{\alpha}{4 \pi \varepsilon_{0}} \rho^{3 / 2} \xi_{0} / 2}, \quad \quad \text { where } \quad \alpha=\alpha_{\text {uni }} / 2
$$




\section{B. Dipoles pointing in $\hat{z}$-direction}

In this case, $\hat{\mathbf{x}}$ and $\hat{\mathbf{y}}$ components of $\mathbf{E}_{\text {dip }}$ will be zero and the dipole moment will be in the form of:

$$
\begin{aligned}
\mathrm{p}_{z} & =\alpha\left[\mathrm{E}_{\mathrm{Ext}, z}+\frac{1}{4 \pi \varepsilon_{0}} \mathrm{p}_{z} \sum_{\mathbf{r}_{n} \neq \mathbf{0}} \frac{-1}{\left(x_{n}^{2}+y_{n}^{2}\right)^{3 / 2}}\right] \\
& =\frac{\alpha}{1+\frac{\alpha}{4 \pi \varepsilon_{0}} \rho^{3 / 2} \xi_{0}} \mathrm{E}_{\mathrm{Ext}, z} .
\end{aligned}
$$

With the boundary condition of the continuity of the electric displacement $\mathrm{E}_{\mathrm{Ext}, z}=$ $\varepsilon_{z z} \mathrm{E}_{\mathrm{Macro}, z}$ and $\mathrm{P}_{z}=c_{\mathrm{d}} \mathrm{p}_{z}=\varepsilon_{0}\left(\varepsilon_{z z}-1\right) \mathrm{E}_{\mathrm{Macro}, z}$, we have:

$$
\begin{aligned}
& \frac{1}{\varepsilon_{z z}}\left(\varepsilon_{z z}-1\right)=\frac{c_{\mathrm{d}} \alpha / \varepsilon_{0}}{1+\frac{\alpha}{4 \pi \varepsilon_{0}} \rho^{3 / 2} \xi_{0}} \\
& \text { i.e, } \quad \frac{1}{\varepsilon_{z z}}=1-\frac{c_{\mathrm{d}} \alpha / \varepsilon_{0}}{1+\frac{\alpha}{4 \pi \varepsilon_{0}} \rho^{3 / 2} \xi_{0}}, \\
& \text { with } \quad \alpha=\alpha_{\text {uni. }} .
\end{aligned}
$$

\subsubsection{Anisotropic DF for 2D Planar Array of Dipoles in a Medium}

Before trying to implement these expressions into anisotropic Mie theory, we first want to generalise them to a much more experimentally relevant case for $\varepsilon_{\mathrm{m}}>1$, i.e. molecules embedded in water $\varepsilon_{\mathrm{m}}=1.33^{2}$, for example. Despite its importance, the generalisation of these expressions could not be found in the literature, and it is not straightforward to obtain them. As previously done in the above section, in a medium, we can account for the local field effects (of the medium molecules onto the adsorbates) by either considering a fully microscopic approach or a mixed microscopic/continuum approach. However, in our theoretical investigation of the problem, the mixed microscopic/continuum approach will be more appropriate, as there are more water molecules than the dye molecules. Next, we will go through the steps of how to obtain the expressions for $\varepsilon_{x y}$ and $\varepsilon_{z z}$ when the dipoles are embedded in a medium.

\section{A. Dipoles isotropically in-plane (smarties-like) continuum approach}

For this approach, as done earlier, we treat the dye molecules as discrete point dipoles embedded in a continuous dielectric medium with permittivity $\varepsilon_{\mathrm{m}}$. If we 
follow Eqs. 4.7 and 4.8, then Eq. 4.13 become:

$$
\begin{aligned}
\mathbf{p}(\mathbf{0}) & =\underline{\alpha}_{\mathrm{d}} \mathbf{e}_{\mathrm{loc}}=\underline{\alpha}_{\mathrm{d}} L_{\mathrm{m}} \mathbf{E}_{\mathrm{loc}} \\
& =\underline{\alpha}_{\mathrm{d}} L_{\mathrm{m}}\left[\mathbf{E}_{\mathrm{Ext}}+L_{\mathrm{m}} \sum_{\mathbf{r}_{n} \neq \mathbf{0}} \mathbf{E}_{\mathrm{dip}}\left(\mathbf{p}, \mathbf{r}_{n}\right)\right] .
\end{aligned}
$$

For dipoles $\mathbf{p}$ embedded in a medium with $\varepsilon_{\mathrm{m}}, \mathbf{E}_{\mathrm{dip}}$ is given as:

$$
\mathbf{E}_{\text {dip }}(\mathbf{p}, \mathbf{r})=-\nabla\left(\frac{\mathbf{p} \cdot \hat{\mathbf{r}}}{4 \pi \varepsilon_{0} \varepsilon_{\mathrm{m}} \mathrm{r}^{2}}\right)=\frac{3(\mathbf{p} \cdot \hat{\mathbf{r}}) \hat{\mathbf{r}}-\mathbf{p}}{4 \pi \varepsilon_{0} \varepsilon_{\mathrm{m}} \mathrm{r}^{3}}
$$

Therefore, for the case where all dipoles are isotropically in-plane oriented, then the dipole moment will become:

$$
\mathrm{p}_{x y}=\frac{\alpha L_{\mathrm{m}}}{1-\frac{\alpha}{4 \pi \varepsilon_{0}} \frac{L_{\mathrm{m}}^{2}}{\varepsilon \mathrm{m}} \rho^{3 / 2} \xi_{0} / 2} \mathrm{E}_{\mathrm{Ext}, x y}
$$

By applying the boundary condition $\mathrm{E}_{\mathrm{Ext}, x y}=\mathrm{E}_{\mathrm{Macro}, x y}$ and the macroscopic polarisation $\mathrm{P}_{x y}=\varepsilon_{0}\left(\varepsilon_{x y}-1\right) \mathrm{E}_{\text {Macro, } x y}=\mathrm{P}_{\mathrm{d}, x y}+\mathrm{P}_{\mathrm{m}, x y}=L_{\mathrm{m}} c_{\mathrm{d}} \mathrm{p}_{x y}+\varepsilon_{0}\left(\varepsilon_{\mathrm{m}}-1\right) \mathrm{E}_{\mathrm{Ext}, x y}$, then $\varepsilon_{x y}$ can be found as:

$$
\begin{gathered}
\varepsilon_{x y}=\varepsilon_{\mathrm{m}}+\frac{L_{\mathrm{m}}^{2} c_{\mathrm{d}} \alpha / \varepsilon_{0}}{1-\frac{\alpha}{4 \pi \varepsilon_{0}} \frac{L_{\mathrm{m}}^{2}}{\varepsilon_{\mathrm{m}}} \rho^{3 / 2} \xi_{0} / 2} \\
\text { with } \quad \alpha=\alpha_{\text {uni }} / 2 .
\end{gathered}
$$

\section{B. Dipoles pointing in $\hat{z}$ direction continuum approach}

If all dipoles point in the $\hat{\mathbf{z}}$-direction, then the dipole moment will become:

$$
\mathrm{p}_{z}=\frac{\alpha L_{\mathrm{m}}}{1+\frac{\alpha}{4 \pi \varepsilon_{0}} \frac{L_{\mathrm{m}}^{2}}{\varepsilon \mathrm{m}} \rho^{3 / 2} \xi_{0}} \mathrm{E}_{\mathrm{Ext}, z} .
$$

In this case, the boundary condition will be: $\varepsilon_{\mathrm{m}} \mathrm{E}_{\mathrm{Ext}, z}=\varepsilon_{z z} \mathrm{E}_{\mathrm{Macro}, z}$, and the macroscopic polarisation $\mathrm{P}_{z}=\varepsilon_{0}\left(\varepsilon_{z z}-1\right) \mathrm{E}_{\text {Macro, } z}=\mathrm{P}_{\mathrm{d}, z}+\mathrm{P}_{\mathrm{m}, z}=L_{\mathrm{m}} c_{\mathrm{d}} \mathrm{p}_{z}+$ $\varepsilon_{0}\left(\varepsilon_{\mathrm{m}}-1\right) \mathrm{E}_{\mathrm{Ext}, z}$. These will lead to:

$$
\begin{gathered}
\frac{1}{\varepsilon_{z z}}=\frac{1}{\varepsilon_{\mathrm{m}}}-\frac{\left(L_{\mathrm{m}}^{2} / \varepsilon_{\mathrm{m}}^{2}\right) c_{\mathrm{d}} \alpha / \varepsilon_{0}}{1+\frac{\alpha}{4 \pi \varepsilon_{0}} \frac{L_{\mathrm{m}}^{2}}{\varepsilon_{\mathrm{m}}}} \rho^{3 / 2} \xi_{0} \\
\text { with } \quad \alpha=\alpha_{\text {uni }} .
\end{gathered}
$$

It is noteworthy that at the limit of low dye concentration, Eqs. 4.24 and 4.26 will reduce to the same expression as in Eq. 3.74. 


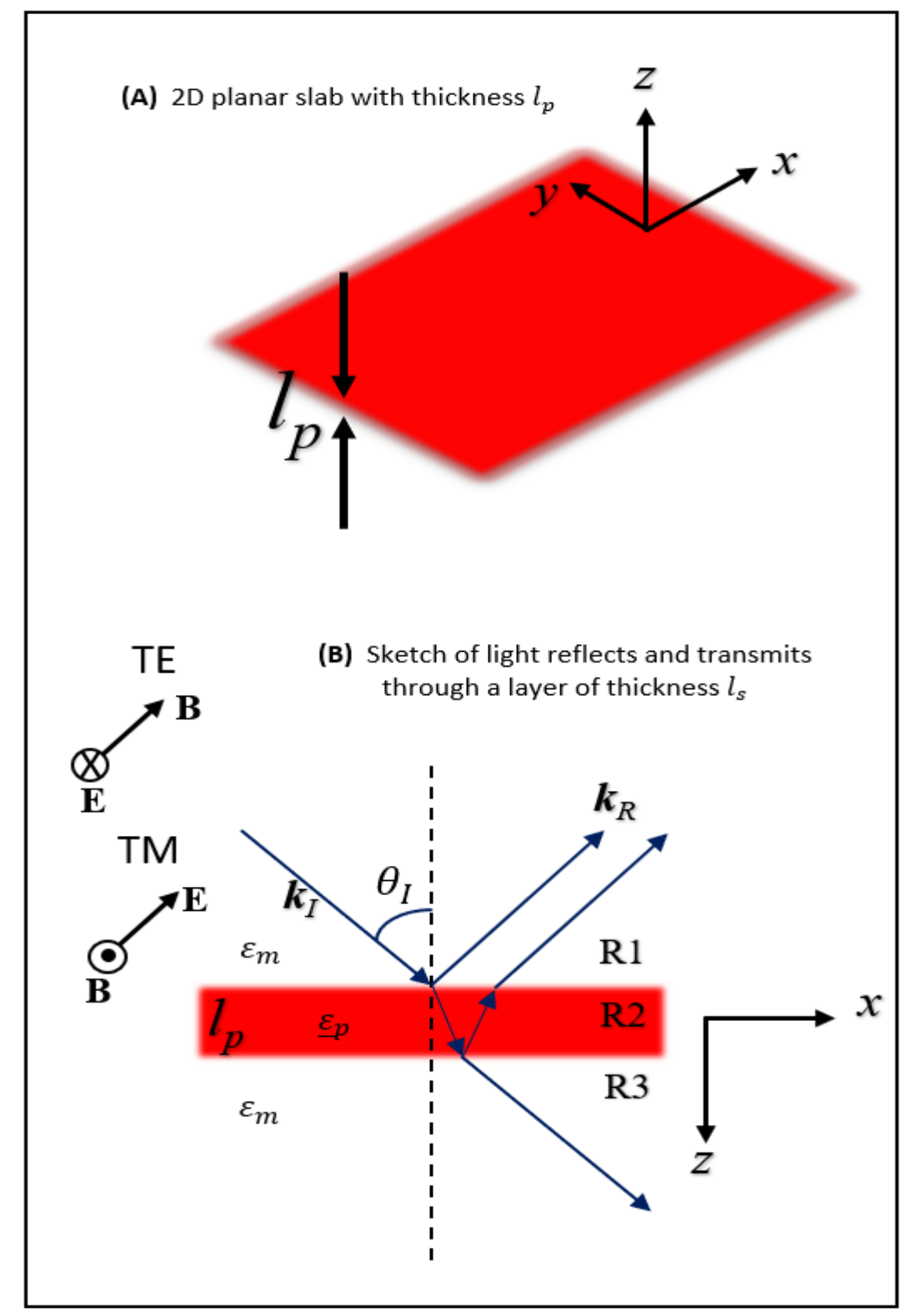

Figure 4.2: Sketch showing in (A) a 2D planar slab of thickness $l_{p}$ and (B) schematic of how light incident on a surface with incident angle of $\theta_{I}$ [with 2 different polarisations p-polarised light (TM) or s-polarised light (TE)] reflect of that surface and transmit through its thickness $l_{s}$. 


\subsection{Anisotropic Fresnel Equations}

Before implementing these effective dielectric functions from the 2D planar array of dipoles into the anisotropic Mie theory, it would be more appropriate to make use of them to predict the optical properties of an anisotropic slab (2D-plane) with a thickness $l_{p}$, as shown in Fig. 4.2, then compare that to the results from the microscopic predictions. To do so, we use Fresnel equations for the anisotropic layer to calculate the absorption coefficients.

The Maxwell equations take the same form as defined in Eq. 3.1. We here consider the dielectric tensor to have its components $\varepsilon_{\mathrm{n}}$ and $\varepsilon_{\mathrm{t}}$, which correspond to the normal and tangential to the surface of the plane, i.e.

$$
\left(\underline{\varepsilon}_{p} \mathbf{E}\right)_{x}=\varepsilon_{\mathrm{t}} \mathrm{E}_{x}, \quad\left(\underline{\varepsilon}_{p} \mathbf{E}\right)_{y}=\varepsilon_{\mathrm{t}} \mathrm{E}_{y}, \quad\left(\underline{\varepsilon}_{p} \mathbf{E}\right)_{z}=\varepsilon_{\mathrm{n}} \mathrm{E}_{z} .
$$

In isotropic media, the dispersion relation $\mathbf{k} \cdot \mathbf{k}=\omega^{2} \mu \varepsilon$ always holds. However, it does not work when the dielectric function is direction-dependent, i.e. in anisotropic media.

\subsubsection{Wave-Vector $\mathrm{k}$ in Anisotropic Media}

We here consider a plane wave (with wave-vector $\mathbf{k}$ in $x z$-plane) incidents on an anisotropic slab with thickness $l_{p}$ as shown in Fig. 4.2, with two different polarisations of light: p-polarised (Transverse Magnetic, TM) and s-polarised (Transverse Electric, TE) lights.

\section{For p-polarised light (TM)}

This is the case where the incident electric field is in the plane of incidence $(x z$ plane), and hence the magnetic field is in the $y$-direction. So, in the region R2 (anisotropic medium), the magnetic field can be expressed as:

$$
\mathbf{B}=\left[\mathrm{B}_{0 \mathrm{~T}} e^{i\left(k_{x} x+k_{z} z\right)}-\mathrm{B}_{0 \mathrm{R} 2} e^{i\left(k_{x} x-k_{z} z\right)}\right] \hat{y} .
$$

By taking the curl of $\mathbf{B}$, the electric field can then be obtained as:

$$
\begin{aligned}
\mathbf{E}= & \frac{i}{\omega \mu \underline{\varepsilon}_{p}} \nabla \times \mathbf{B}=\frac{i}{\omega \mu \underline{\varepsilon}_{p}}\left[-\frac{\partial \mathrm{B}_{y}}{\partial z} \hat{x}+\frac{\partial \mathrm{B}_{y}}{\partial x} \hat{z}\right] \\
= & \frac{k_{z}}{\omega \mu \varepsilon_{t}}\left(\mathrm{~B}_{0 \mathrm{~T}} e^{i\left(k_{x} x+k_{z} z\right)}+\mathrm{B}_{0 \mathrm{R} 2} e^{i\left(k_{x} x-k_{z} z\right)}\right) \hat{x} \\
& -\frac{k_{z}}{\omega \mu \varepsilon_{n}}\left(\mathrm{~B}_{0 \mathrm{~T}} e^{i\left(k_{x} x+k_{z} z\right)}-\mathrm{B}_{0 \mathrm{R} 2} e^{i\left(k_{x} x-k_{z} z\right)}\right) \hat{z} .
\end{aligned}
$$


If we take the curl of $\mathbf{E}$, it will lead to:

$$
\begin{aligned}
\mathbf{B} & =\frac{1}{i \omega} \nabla \times \mathbf{E}=\frac{1}{i \omega}\left[-\frac{\partial \mathrm{E}_{z}}{\partial x}+\frac{\partial \mathrm{E}_{x}}{\partial x}\right] \hat{y} \\
& =\frac{1}{\omega^{2} \mu}\left(\frac{k_{x}^{2}}{\varepsilon_{n}}+\frac{k_{z}^{2}}{\varepsilon_{t}}\right)\left[\mathrm{B}_{0 \mathrm{~T}} e^{i\left(k_{x} x+k_{z} z\right)}-\mathrm{B}_{0 \mathrm{R} 2} e^{i\left(k_{x} x-k_{z} z\right)}\right] \hat{y} .
\end{aligned}
$$

By matching the coefficients of Eq. 4.27 and Eq. 4.29, we get:

$$
\left(k_{z}^{\mathrm{TM}}\right)^{2}=\left(\varepsilon_{t} / \varepsilon_{0}\right) k_{0}^{2}-\left(\varepsilon_{t} / \varepsilon_{n}\right) k_{x}^{2}
$$

where $k_{0}=\omega \sqrt{\mu \varepsilon_{0}}=2 \pi / \lambda$ and $k_{x}=k_{0} \sqrt{\varepsilon_{\mathrm{m}}} \sin \theta_{I}$. If $\varepsilon_{x}=\varepsilon_{z}$, then the dispersion relation is modified.

\section{For s-polarised light (TE)}

Similarly, for s-polarised light, the incident magnetic field is now in the plane of incidence ( $x z$-plane), and therefore the electric field is in the $y$-direction. So, in the region $\mathrm{R} 2$ (anisotropic medium), the electric field can be expressed as:

$$
\mathbf{E}=\left[\mathrm{E}_{0 \mathrm{~T}} e^{i\left(k_{x} x+k_{z} z\right)}+\mathrm{E}_{0 \mathrm{R} 2} e^{i\left(k_{x} x-k_{z} z\right)}\right] \hat{y} .
$$

If we proceed the same steps as done in the p-polarised light, it will lead to:

$$
\left(k_{z}^{\mathrm{TE}}\right)^{2}=\left(\varepsilon_{t} / \varepsilon_{0}\right) k_{0}^{2}-k_{x}^{2}
$$

From Eqs. 4.30 and 4.32, the wave-vector $\left(k_{z}\right)$ is shown to have different relations for the two different polarised lights for anisotropic media. This would lead to differences in the optical spectrum, for example, the absorption spectrum for the two polarisations of light.

\subsubsection{Electric and Magnetic Fields}

In this section, we define the fields in each region (R1, R2, and R3, refer to Fig. 4.2), for p-polarised and s-polarised lights.

\section{For p-polarised light (TM)}

$$
\begin{aligned}
& \mathbf{B}_{\mathrm{R} 1}^{\mathrm{TM}}=\left[\mathrm{B}_{0 \mathrm{I}}^{\mathrm{TM}} e^{i k_{I}\left(\sin \theta_{I} x+\cos \theta_{I} z\right)}-\mathrm{B}_{0 \mathrm{R}}^{\mathrm{TM}} e^{i k_{I}\left(\sin \theta_{I} x-\cos \theta_{I} z\right)}\right] \hat{y} . \\
& \mathbf{B}_{\mathrm{R} 2}^{\mathrm{TM}}=\left[\mathrm{B}_{0 \mathrm{~T}}^{\mathrm{TM}} e^{i\left(k_{x} x+k_{z}^{\mathrm{TM}} z\right)}-\mathrm{B}_{0 \mathrm{R} 2}^{\mathrm{TM}} e^{i\left(k_{x} x-k_{z}^{\mathrm{TM}} z\right)}\right] \hat{y} . \\
& \mathbf{B}_{\mathrm{R} 3}^{\mathrm{TM}}=\mathrm{B}_{0 \mathrm{~T} 2}^{\mathrm{TM}} e^{i k_{I}\left(\sin \theta_{I} x+\cos \theta_{I} z\right)} \hat{y} .
\end{aligned}
$$


The corresponding electric fields for each region can be found by taking the curl of their respective magnetic fields, as:

$$
\mathbf{E}_{\mathrm{R} 1, \mathrm{R} 3}^{\mathrm{TM}}=\frac{i}{\omega \mu \varepsilon_{\mathrm{m}}} \nabla \times \mathbf{B}_{\mathrm{R} 1, \mathrm{R} 3}^{\mathrm{TM}}, \quad \mathbf{E}_{\mathrm{R} 2}^{\mathrm{TM}}=\frac{i}{\omega \mu \varepsilon_{p}} \nabla \times \mathbf{B}_{\mathrm{R} 2}^{\mathrm{TM}} .
$$

\section{For s-polarised light (TE)}

$$
\begin{aligned}
& \mathbf{E}_{\mathrm{R} 1}^{\mathrm{TE}}=\left[\mathrm{E}_{0 \mathrm{I}}^{\mathrm{TE}} e^{i k_{I}\left(\sin \theta_{I} x+\cos \theta_{I} z\right)}+\mathrm{E}_{0 \mathrm{R}}^{\mathrm{TE}} e^{i k_{I}\left(\sin \theta_{I} x-\cos \theta_{I} z\right)}\right] \hat{y} . \\
& \mathbf{E}_{\mathrm{R} 2}^{\mathrm{TE}}=\left[\mathrm{E}_{0 \mathrm{~T}}^{\mathrm{TE}} e^{i\left(k_{x} x+k_{z}^{\mathrm{TE}} z\right)}+\mathrm{E}_{0 \mathrm{R} 2}^{\mathrm{TE}} e^{i\left(k_{x} x-k_{z}^{\mathrm{TE}} z\right)}\right] \hat{y} . \\
& \mathbf{E}_{\mathrm{R} 3}^{\mathrm{TE}}=\mathrm{E}_{0 \mathrm{~T} 2}^{\mathrm{TE}} e^{i k_{I}\left(\sin \theta_{I} x+\cos \theta_{I} z\right)} \hat{y} .
\end{aligned}
$$

The corresponding magnetic fields for each region can be found by taking the curl of their respective electric fields, as:

$$
\mathbf{B}_{\mathrm{R} 1, \mathrm{R} 2, \mathrm{R} 3}^{\mathrm{TE}}=\frac{-i}{\omega} \nabla \times \mathbf{E}_{\mathrm{R} 1, \mathrm{R} 2, \mathrm{R} 3}^{\mathrm{TE}} .
$$

\subsubsection{Absorption Coefficients and Boundary Conditions}

\section{Reflection, Transmission, and Absorption coefficients}

The reflection and transmission coefficients can be found as in the Fresnel equations, which are given as:

$$
R=\left|\frac{\mathrm{E}_{0 \mathrm{R}}^{\mathrm{TE}}}{\mathrm{E}_{0 \mathrm{I}}^{\mathrm{TE}}}\right|^{2}=\left|\frac{\mathrm{B}_{0 \mathrm{R}}^{\mathrm{TM}}}{\mathrm{B}_{0 \mathrm{I}}^{\mathrm{TM}}}\right|^{2}, \quad T=\left|\frac{\mathrm{E}_{0 \mathrm{~T} 2}^{\mathrm{TE}}}{\mathrm{E}_{0 \mathrm{I}}^{\mathrm{TE}}}\right|^{2}=\left|\frac{\mathrm{B}_{0 \mathrm{~T} 2}^{\mathrm{TM}}}{\mathrm{B}_{0 \mathrm{I}}^{\mathrm{TM}}}\right|^{2} .
$$

The absorption coefficient can then be calculated as:

$$
A=1-(R+T) \text {. }
$$

\section{Boundary Conditions for p-polarised light}

At the interfaces $z=0$ and $z=l_{p}$, the continuity of the fields are the boundary conditions (note that $x=0$ ), which are

$$
\begin{aligned}
\text { At } z & =l_{s}, & z & =0 \\
\left(\varepsilon_{\mathrm{m}} \mathbf{E}_{\mathrm{R} 3}^{\mathrm{TM}}\right)_{z} & =\left(\underline{\varepsilon}_{p} \mathbf{E}_{\mathrm{R} 2}^{\mathrm{TM}}\right)_{z}, & \left(\underline{\varepsilon}_{p} \mathbf{E}_{\mathrm{R} 2}^{\mathrm{TM}}\right)_{z} & =\left(\varepsilon_{\mathrm{m}} \mathbf{E}_{\mathrm{R} 1}^{\mathrm{TM}}\right)_{z}, \\
\left(\mathbf{E}_{\mathrm{R} 3}^{\mathrm{TM}}\right)_{x} & =\left(\mathbf{E}_{\mathrm{R} 2}^{\mathrm{TM}}\right)_{x}, & \left(\mathbf{E}_{\mathrm{R} 2}^{\mathrm{TM}}\right)_{x} & =\left(\mathbf{E}_{\mathrm{R} 1}^{\mathrm{TM}}\right)_{x}, \\
\left(\mathbf{B}_{\mathrm{R} 3}^{\mathrm{TM}}\right)_{y} & =\left(\mathbf{B}_{\mathrm{R} 2}^{\mathrm{TM}}\right)_{y}, & \left(\mathbf{B}_{\mathrm{R} 2}^{\mathrm{TM}}\right)_{y} & =\left(\mathbf{B}_{\mathrm{R} 1}^{\mathrm{TM}}\right)_{y} .
\end{aligned}
$$


So, from the boundary condition at $z=l_{s}$ we get:

$$
\begin{aligned}
\frac{k_{I} \sin \theta_{I}}{\omega \mu} \mathrm{B}_{0 \mathrm{~T} 2}^{\mathrm{TM}} e^{i k_{I} \cos \theta d} & =\frac{k_{x}}{\omega \mu}\left(\mathrm{B}_{0 \mathrm{~T}}^{\mathrm{TM}} e^{i k_{z}^{\mathrm{TM}} d}-\mathrm{B}_{0 \mathrm{R} 2}^{\mathrm{TM}} e^{-i k_{z}^{\mathrm{TM}} d}\right) \\
\frac{k_{I} \sin \theta_{I}}{\omega \mu \varepsilon_{\mathrm{m}}} \mathrm{B}_{0 \mathrm{~T} 2}^{\mathrm{TM}} e^{i k_{I} \cos \theta d} & =\frac{k_{z}^{\mathrm{TM}}}{\omega \mu \varepsilon_{t}}\left(\mathrm{~B}_{0 \mathrm{~T}}^{\mathrm{TM}} e^{i k_{z}^{\mathrm{TM}} d}+\mathrm{B}_{0 \mathrm{R} 2}^{\mathrm{TM}} e^{-i k_{z}^{\mathrm{TM}} d}\right) \\
\mathrm{B}_{0 \mathrm{~T} 2}^{\mathrm{TM}} e^{i k_{I} \cos \theta d} & =\mathrm{B}_{0 \mathrm{~T}}^{\mathrm{TM}} e^{i k_{z}^{\mathrm{TM}} d}-\mathrm{B}_{0 \mathrm{R} 2}^{\mathrm{TM}} e^{-i k_{z}^{\mathrm{TM}} d} .
\end{aligned}
$$

From these equations, the following can be deduced:

$$
\begin{aligned}
\mathrm{B}_{0 \mathrm{R} 2}^{\mathrm{TM}} & =\frac{\left(k_{x} / k_{z}^{\mathrm{TM}}\right)-\left(\varepsilon_{\mathrm{m}} / \varepsilon_{\mathrm{t}}\right) \tan \theta_{I}}{\left(k_{x} / k_{z}^{\mathrm{TM}}\right)+\left(\varepsilon_{\mathrm{m}} / \varepsilon_{\mathrm{t}}\right) \tan \theta_{I}} e^{2 i k_{z}^{\mathrm{TM}} d} \mathrm{~B}_{0 \mathrm{~T}}^{\mathrm{TM}}=\alpha^{\mathrm{TM}} \mathrm{B}_{0 \mathrm{~T}}^{\mathrm{TM}} . \\
\mathrm{B}_{0 \mathrm{~T} 2}^{\mathrm{TM}} & =\frac{\left(2 \varepsilon_{\mathrm{m}} / \varepsilon_{\mathrm{t}}\right) \tan \theta_{I}}{\left(k_{x} / k_{z}^{\mathrm{TM}}\right)+\left(\varepsilon_{\mathrm{m}} / \varepsilon_{\mathrm{t}}\right) \tan \theta_{I}} e^{i\left(k_{z}^{\mathrm{TM}}-k_{i} \cos \theta_{I}\right) d} \mathrm{~B}_{0 \mathrm{~T}}^{\mathrm{TM}}=\beta^{\mathrm{TM}} \mathrm{B}_{0 \mathrm{~T}}^{\mathrm{TM}}
\end{aligned}
$$

And the boundary condition at $z=0$ gives:

$$
\begin{aligned}
\frac{k_{I} \sin \theta_{I}}{\omega \mu}\left(\mathrm{B}_{0 \mathrm{I}}^{\mathrm{TM}}-\mathrm{B}_{0 \mathrm{R}}^{\mathrm{TM}}\right) & =\frac{k_{x}}{\omega \mu}\left(\mathrm{B}_{0 \mathrm{~T}}^{\mathrm{TM}}-\mathrm{B}_{0 \mathrm{R} 2}^{\mathrm{TM}}\right) \\
\frac{k_{I} \sin \theta_{I}}{\omega \mu \varepsilon_{\mathrm{m}}}\left(\mathrm{B}_{0 \mathrm{I}}^{\mathrm{TM}}+\mathrm{B}_{0 \mathrm{R}}^{\mathrm{TM}}\right) & =\frac{k_{z}^{\mathrm{TM}}}{\omega \mu \varepsilon_{\mathrm{t}}}\left(\mathrm{B}_{0 \mathrm{~T}}^{\mathrm{TM}}+\mathrm{B}_{0 \mathrm{R} 2}^{\mathrm{TM}}\right) \\
\mathrm{B}_{0 \mathrm{I}}^{\mathrm{TM}}-\mathrm{B}_{0 \mathrm{R}}^{\mathrm{TM}} & =\mathrm{B}_{0 \mathrm{~T}}^{\mathrm{TM}}-\mathrm{B}_{0 \mathrm{R} 2}^{\mathrm{TM}} .
\end{aligned}
$$

These equations with Eqs. 4.47 and 4.48 will give:

$$
\begin{aligned}
\frac{\mathrm{B}_{0 \mathrm{R}}^{\mathrm{TM}}}{\mathrm{B}_{0 \mathrm{I}}^{\mathrm{TM}}} & =\frac{\left(\varepsilon_{\mathrm{m}} / \varepsilon_{\mathrm{t}}\right) \tan \theta_{I}-\left[k_{x}\left(1-\alpha^{\mathrm{TM}}\right)\right] /\left[k_{z}^{\mathrm{TM}}\left(1+\alpha^{\mathrm{TM}}\right)\right]}{\left(\varepsilon_{\mathrm{m}} / \varepsilon_{\mathrm{t}}\right) \tan \theta_{I}+\left[k_{x}\left(1-\alpha^{\mathrm{TM}}\right)\right] /\left[k_{z}^{\mathrm{TM}}\left(1+\alpha^{\mathrm{TM}}\right)\right]} \\
\frac{\mathrm{B}_{0 \mathrm{~T} 2}^{\mathrm{TM}}}{\mathrm{B}_{0 \mathrm{I}}^{\mathrm{TM}}} & =\beta^{\mathrm{TM}} \frac{2 k_{x} /\left[k_{z}^{\mathrm{TM}}\left(1+\alpha^{\mathrm{TM}}\right)\right]}{\left(\varepsilon_{\mathrm{m}} / \varepsilon_{\mathrm{t}}\right) \tan \theta_{I}+\left[k_{x}\left(1-\alpha^{\mathrm{TM}}\right)\right] /\left[k_{z}^{\mathrm{TM}}\left(1+\alpha^{\mathrm{TM}}\right)\right]} .
\end{aligned}
$$

\section{Boundary Conditions for s-polarised light}

$$
\begin{array}{rlrl}
\text { At } z & =l_{s}, & z & =0 \\
\left(\mathbf{B}_{\mathrm{R} 3}^{\mathrm{TE}}\right)_{z} & =\left(\mathbf{B}_{\mathrm{R} 2}^{\mathrm{TE}}\right)_{z}, & & \left(\mathbf{B}_{\mathrm{R} 2}^{\mathrm{TE}}\right)_{z}=\left(\mathbf{B}_{\mathrm{R} 1}^{\mathrm{TE}}\right)_{z}, \\
\left(\mathbf{B}_{\mathrm{R} 3}^{\mathrm{TE}}\right)_{x}=\left(\mathbf{B}_{\mathrm{R} 2}^{\mathrm{TE}}\right)_{x}, & \left(\mathbf{B}_{\mathrm{R} 2}^{\mathrm{TE}}\right)_{x}=\left(\mathbf{B}_{\mathrm{R} 1}^{\mathrm{TE}}\right)_{x}, \\
\left(\mathbf{E}_{\mathrm{R} 3}^{\mathrm{TE}}\right)_{y}=\left(\mathbf{E}_{\mathrm{R} 2}^{\mathrm{TE}}\right)_{y}, & \left(\mathbf{E}_{\mathrm{R} 2}^{\mathrm{TE}}\right)_{y}=\left(\mathbf{E}_{\mathrm{R} 1}^{\mathrm{TE}}\right)_{y} .
\end{array}
$$


With these boundary conditions, one can derive the following:

$$
\begin{aligned}
\frac{\mathrm{E}_{0 \mathrm{R}}^{\mathrm{TE}}}{\mathrm{E}_{0 \mathrm{I}}^{\mathrm{TE}}} & =\frac{k_{x}\left(1+\alpha^{\mathrm{TE}}\right)-k_{z}^{\mathrm{TE}}\left(1-\alpha^{\mathrm{TE}}\right) \tan \theta_{I}}{k_{x}\left(1+\alpha^{\mathrm{TE}}\right)+k_{z}^{\mathrm{TE}}\left(1-\alpha^{\mathrm{TE}}\right) \tan \theta_{I}} \\
\frac{\mathrm{E}_{0 \mathrm{~T} 2}^{\mathrm{TE}}}{\mathrm{E}_{0 \mathrm{I}}^{\mathrm{TE}}} & =\beta^{\mathrm{TE}} \frac{2 k_{x}}{k_{x}\left(1+\alpha^{\mathrm{TE}}\right)+k_{z}^{\mathrm{TE}}\left(1-\alpha^{\mathrm{TE}}\right) \tan \theta_{I}},
\end{aligned}
$$

where

$$
\begin{aligned}
\alpha^{\mathrm{TE}} & =\frac{k_{z}^{\mathrm{TE}} \tan \theta_{I}-k_{x}}{k_{z}^{\mathrm{TE}} \tan \theta_{I}+k_{x}} e^{2 i k_{z}^{\mathrm{TE}} d} \\
\beta^{\mathrm{TE}} & =\frac{2 k_{z}^{\mathrm{TE}} \tan \theta_{I}}{k_{z}^{\mathrm{TE}} \tan \theta_{I}+k_{x}} e^{i\left(k_{z}^{\mathrm{TE}}-k_{I} \cos \theta_{I}\right) d} .
\end{aligned}
$$

With these anisotropic Fresnel equations, we can use them as the effective medium model solutions for anisotropic planar layer (with effective dielectric functions) and compare that to analytic solutions from microscopic model (in the next section).

\subsection{An Improved Microscopic Model with Radiative Cor- rection Term}

\subsubsection{In Vacuum}

The calculation of the reflectance and transmittance of an infinite square lattice (including the retarded dipole-dipole interaction between all particles) has been studied by Vlieger [72]. The author has derived analytic expressions for a 2D planar layer of isotropic polarisable dipole in vacuum. A similar derivation can be carried out for the two types of anisotropic polarisable dipoles related to our work. The dipoles are arranged in a square array with density $\rho$ (no. dipoles/unit area). The dipoles are in $x y$ plane and we consider incident p-polarised light at an angle $\theta_{I}$ from the normal ( $z$ axis). For the either uni-axial with random orientation or isotropic inplane polarisabilities $\underline{\alpha}=\alpha_{\mathrm{d}}^{\|}(\hat{x} \otimes \hat{x}+\hat{y} \otimes \hat{y})$ dipole, a derivation similar to Ref. [72] results in the following absorption coefficient (absorption cross-section per unit area) in vacuum as:

$$
A_{v a c}^{\|}=\frac{k_{0} \rho}{\varepsilon_{0}} \frac{\Im\left(\alpha_{\mathrm{d}}^{\|}\right)}{\left|1-\frac{\alpha_{\mathrm{d}}^{\|}}{4 \pi \varepsilon_{0}}\left[\rho^{3 / 2} \xi_{0} / 2+2 i \pi k_{0} \rho \cos \theta_{I}\right]\right|^{2}} \cos \theta_{I},
$$

where $k_{0}=2 \pi / \lambda$, and the second term in the bracket [] in the denominator is akin to the retardation correction to the dipole-dipole interactions a radiative correction 
term [73]. For perpendicular dipoles, i.e $\underline{\alpha}=\alpha_{\mathrm{d}}^{\perp} \hat{z} \otimes \hat{z}$, we simply get:

$$
A_{v a c}^{\perp}=\frac{k_{0} \rho}{\varepsilon_{0}} \frac{\Im\left(\alpha_{\mathrm{d}}^{\perp}\right)}{\left|1+\frac{\alpha_{\mathrm{d}}^{\perp}}{4 \pi \varepsilon_{0}}\left[\rho^{3 / 2} \xi_{0}-2 i \pi k_{0} \rho \frac{\sin ^{2} \theta_{I}}{\cos \theta_{I}}\right]\right|^{2}} \frac{\sin ^{2} \theta_{I}}{\cos \theta_{I}} .
$$

For fully isotropic dipoles with $\underline{\alpha}=\alpha_{d} \underline{I}$, we then have $A_{v a c}^{*}=A_{v a c}^{\|}+A_{v a c}^{\perp}$, which is equivalent to Eq. 68 in Ref. [72].

\subsubsection{In Medium}

We are now going to revisit the microscopic model of an anisotropic layer of molecules embedded in a medium, as done in section (2.4.1). However, this will be done in 2D (Cartesian coordinates) instead of 3D spherical coordinates. We here consider the same scenario as defined in Ref. [72], where the p-polarised light (k) is coming at an angle $\theta_{I}$ with respect to the normal, z-axis, i.e. $\mathbf{E}_{\mathrm{Inc}}=\mathrm{E}_{\mathrm{Inc}}\left(\cos \theta_{I}, 0, \sin \theta_{I}\right)$. Then the incident power on surface $A_{p}$ is given by:

$$
P_{\text {Inc }}=A_{p} \frac{\varepsilon_{0} c}{2} \sqrt{\varepsilon_{\mathrm{m}}}\left|\mathbf{E}_{\text {Inc }}\right|^{2} \cos \theta_{I}=A_{p} S_{\text {Inc }} .
$$

If all dipoles are in-plane isotropic, $\|$ (or perpendicular to the plane, $\perp$ ), then:

$$
\begin{aligned}
& \mathrm{p}_{\text {static }}^{\|}=\alpha_{\mathrm{d}}^{\|} \mathrm{E}_{\mathrm{Loc}, x}=\frac{\alpha_{\mathrm{d}}^{\|} L_{\mathrm{m}}}{1-\frac{\alpha_{\mathrm{d}}^{\|}}{4 \pi \varepsilon_{0}} \frac{L_{\mathrm{m}}^{2}}{\varepsilon_{\mathrm{m}}} \rho^{3 / 2} \xi_{0} / 2} \mathrm{E}_{\text {Inc }} \cos \theta_{I}, \\
& \mathrm{p}_{\text {static }}^{\perp}=\alpha_{\mathrm{d}}^{\perp} \mathrm{E}_{\mathrm{Loc}, z}=\frac{\alpha_{\mathrm{d}}^{\perp} L_{\mathrm{m}}}{1+\frac{\alpha_{\mathrm{d}}^{\perp}}{4 \pi \varepsilon_{0}} \frac{L_{\mathrm{m}}^{2}}{\varepsilon_{\mathrm{m}}} \rho^{3 / 2} \xi_{0}}\left(-\mathrm{E}_{\mathrm{Inc}} \sin \theta_{I}\right) .
\end{aligned}
$$

The absorption cross-section for $N$ molecules can then be expressed as::

$$
\begin{aligned}
& A_{\text {static }}=\frac{P_{\mathrm{Abs}}}{S_{\mathrm{Inc}}}=\frac{k \rho}{\varepsilon_{0}} \frac{L_{\mathrm{m}}^{2}}{\varepsilon_{\mathrm{m}}} \frac{\Im\left[\mathbf{p} \cdot \mathbf{E}_{\mathrm{Loc}}^{*}\right]}{\left|\mathbf{E}_{\mathrm{Inc}}\right|^{2} \cos \theta_{I}} . \\
& A_{\text {static }}^{\|}=\frac{k \rho}{\varepsilon_{0}} \frac{L_{\mathrm{m}}^{2}}{\varepsilon_{\mathrm{m}}} \frac{\Im\left(\alpha_{\mathrm{d}}^{\|}\right)}{\left|1-\frac{\alpha_{\mathrm{d}}^{\|}}{4 \pi \varepsilon_{0}} \frac{L_{\mathrm{m}}^{2}}{\varepsilon_{\mathrm{m}}} \rho^{3 / 2} \xi_{0} / 2\right|^{2}} \cos \theta_{I} \\
& A_{\text {static }}^{\perp}=\frac{k \rho}{\varepsilon_{0}} \frac{L_{\mathrm{m}}^{2}}{\varepsilon_{\mathrm{m}}} \frac{\Im\left(\alpha_{\mathrm{d}}^{\perp}\right)}{\left|1+\frac{\alpha_{\mathrm{d}}^{\perp}}{4 \pi \varepsilon_{0}} \frac{L_{\mathrm{m}}^{2}}{\varepsilon_{\mathrm{m}}} \rho^{3 / 2} \xi_{0}\right|^{2}} \frac{\sin ^{2} \theta_{I}}{\cos \theta_{I}} .
\end{aligned}
$$

where $k=2 \pi \sqrt{\varepsilon_{\mathrm{m}}} / \lambda$. Taking these corrections into account and running through the derivations, as done in Ref. [72] again, Eqs. 4.59 and 4.60 can be generalised as:

$$
\begin{aligned}
A^{\|} & =\frac{k \rho}{\varepsilon_{0}} \frac{L_{\mathrm{m}}^{2}}{\varepsilon_{\mathrm{m}}} \frac{\Im\left(\alpha_{\mathrm{d}}^{\|}\right)}{\left|1-\frac{\alpha_{\mathrm{d}}^{\|}}{4 \pi \varepsilon_{0}} \frac{L_{\mathrm{m}}^{2}}{\varepsilon_{\mathrm{m}}}\left[\rho^{3 / 2} \xi_{0} / 2+2 i \pi k \rho \cos \theta_{I}\right]\right|^{2}} \cos \theta_{I}, \\
A^{\perp} & =\frac{k \rho}{\varepsilon_{0}} \frac{L_{\mathrm{m}}^{2}}{\varepsilon_{\mathrm{m}}} \frac{\Im\left(\alpha_{\mathrm{d}}^{\perp}\right)}{\left|1+\frac{\alpha_{\mathrm{d}}^{\perp}}{4 \pi \varepsilon_{0}} \frac{L_{\mathrm{m}}^{2}}{\varepsilon_{\mathrm{m}}}\left[\rho^{3 / 2} \xi_{0}-2 i \pi k \rho \frac{\sin ^{2} \theta_{I}}{\cos \theta_{I}}\right]\right|^{2}} \frac{\sin ^{2} \theta_{I}}{\cos \theta_{I}} .
\end{aligned}
$$




\subsubsection{Anisotropic EMM planar vs MM predictions}

To seek for the comparison between the two models (anisotropic 2D-EMM and the improved MM), we here consider the special case of $45^{\circ}$ incident with p-polarised light, as this will excite both perpendicular and parallel excitations (other different incident-angles would result in the same conclusions). For isotropically in-plane case, we have:

$$
\varepsilon_{\mathrm{t}}^{\|}=\varepsilon_{x y} \quad \varepsilon_{\mathrm{n}}^{\|}=\varepsilon_{\mathrm{m}} \quad \alpha_{\mathrm{d}}^{\|}=\alpha_{\text {uni }} / 2,
$$

and for perpendicular case, we have:

$$
\varepsilon_{\mathrm{t}}^{\perp}=\varepsilon_{\mathrm{m}} \quad \varepsilon_{\mathrm{n}}^{\perp}=\varepsilon_{z z} \quad \alpha_{\mathrm{d}}^{\|}=\alpha_{\mathrm{uni}}
$$

As presented in Fig. 4.3, the results from both calculations are in perfect agreement. Although we have not shown here the results for molecules with a fully isotropic response (or randomly pointed as in Fig. 3.2 a.), it is worth noting that the anisotropic EMM is more suited than normal isotropic Fresnel equations. This is due to the fact that the interfaces break the symmetry of the molecular response and an anisotropic dielectric tensor is therefore still required, given as:

$$
\varepsilon_{\mathrm{t}}^{\text {Iso }}=\varepsilon_{x y} \quad \quad \varepsilon_{\mathrm{n}}^{\text {Iso }}=\varepsilon_{z z} \quad \alpha_{\mathrm{d}}^{\|}=\alpha_{\text {uni }} / 3 .
$$

The conclusion that we want to draw from this is that the anisotropic EMM planar works extremely well with our derived anisotropic dielectric function. This is shown via Fig. 4.3.

\subsection{Implementing Anisotropic DF into Anisotropic Mie Theory}

To apply the derived anisotropic DF Eqs. 4.24 and 4.26 into Mie theory for the anisotropic shell (the dielectric tensor for the shell), we consider wrapping the $2 \mathrm{D}$ planar sheet around a sphere, as depicted in Fig. 4.4. As long as only the local fields are captured (in other words, when the radius of the inside medium is large enough), so there will be no effect from antipodal points, then wrapping a 2D planar array of dipoles around the metallic nano-sphere will work. For both cases of interest, we define the components of the dielectric tensor as in Eqs. 4.68 and 4.69. 


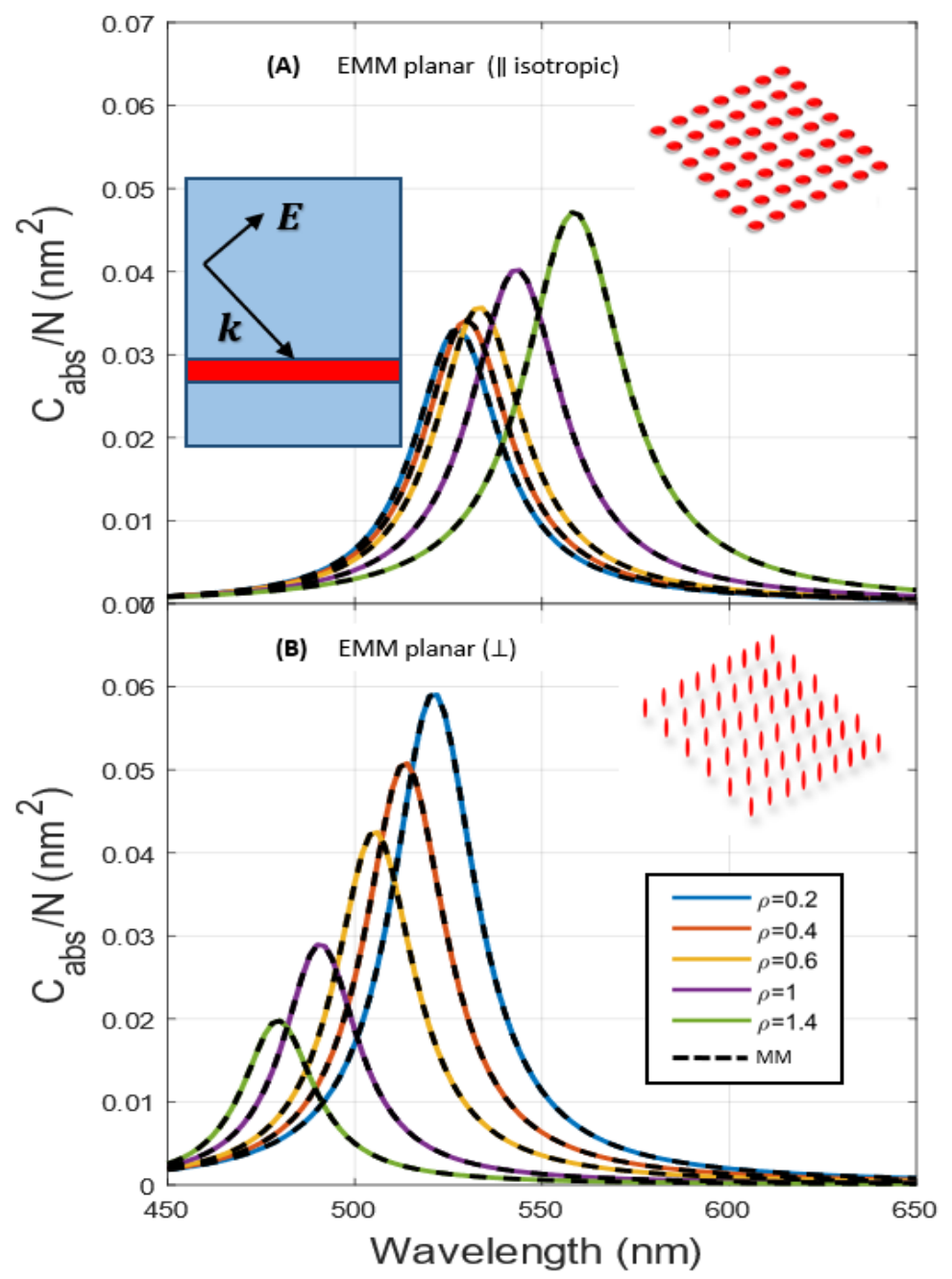

Figure 4.3: Comparison between anisotropic EMM planar predictions for an infinite planar array (with thickness $l_{p}=1 \mathrm{~nm}$ ) of in-plane isotropic (A), or perpendicular (B) dipoles in water and the analytic solutions from the improved microscopic model given in eqs. 4.66 and 4.67 (dashed lines). 


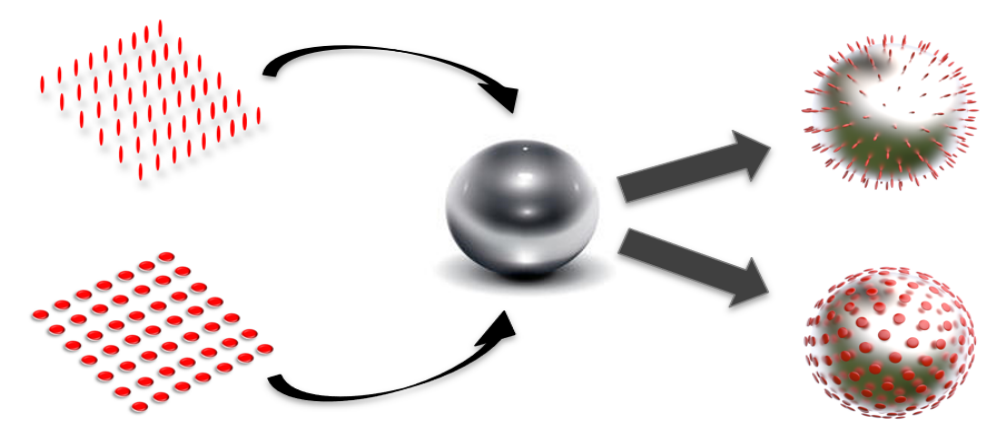

Figure 4.4: Schematic of implementations of the 2D planar array of dipoles onto a silver sphere to get the 2 cases where the molecules adsorbed onto the sphere with normal or radial, $\perp$ (tangential or isotropically in-plane, $\|$ ) to the sphere's surface.

\subsubsection{Comparison to GCDM}

By implementing these into anisotropic Mie theory, the coverage dependent predictions of the absorption cross-section for the system can now be compared to the results of GCDM and this is illustrated in Fig. 4.5. The results of the GCDM predict different shifts for different dye-orientation. As the dye-coverage increases, GCDM predicts blue (red) shifts for radially (isotropically in-plane) oriented dipoles. The results from anisotropic Mie theory with the effective dielectric functions also predict the same shifts. Although the shift in the dye resonance does not depend on the thickness $l_{s}$, the intensities at the dye-resonance decrease as a function of $l_{s}$. Especially for the isotropically in-plane dipoles, we notice that the intensities of the differential absorption cross-sections more dramatically depending on the thickness of the shell compared to the radial orientation case. The results show that by increasing the thickness of the shell $\left(l_{s}\right)$ from $1 \mathrm{~nm}$ to $1.9 \mathrm{~nm}$, the differential absorption cross-section decreases by $\sim 13 \%$ for the radial orientation and $\sim 31.5 \%$ for the isotropically in-plane case. To be able to explain this, we will consider how the electric-field intensity varies as distance $r$ away from the surface.

\subsection{2 $\mathrm{M}_{\mathrm{loc}}$ as a Function of Distance Away from the Sphere}

For the consideration of how $\mathbf{M}_{\text {loc }}$ varies as a function of distance away from the sphere, we can begin by assuming a sphere of radius $a$ as a point dipole. When it is excited by an incident electric field $\mathbf{E}_{0}$ it will induce an oscillating dipole with a 

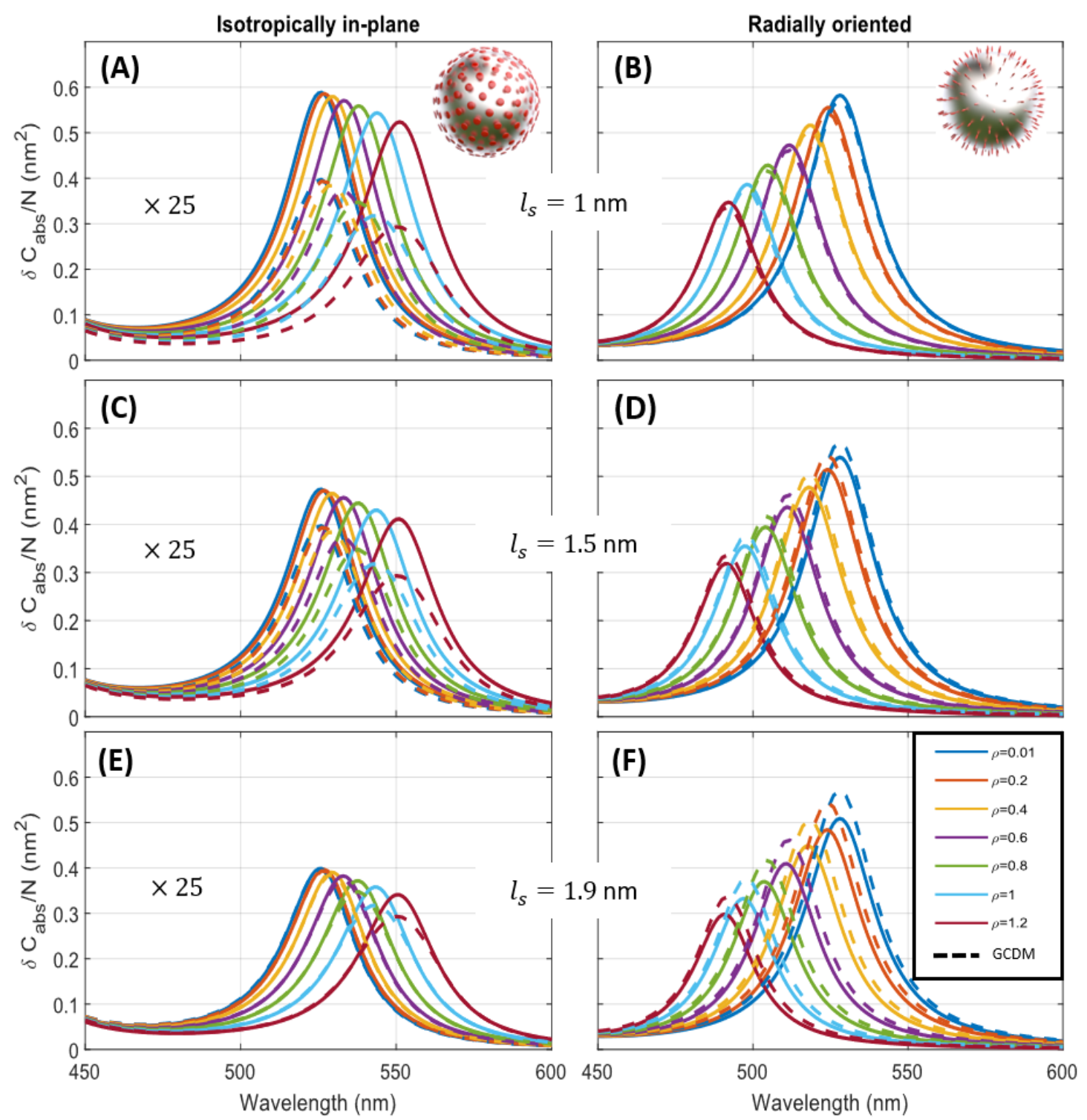

Figure 4.5: The effect of dye-concentration on the optical differential absorption crosssection using Mie theory (solid line) with shell-thickness dependent (A \& B) $l_{s}=1 \mathrm{~nm}$, $(\mathrm{C} \& \mathrm{D}) l_{s}=1.5 \mathrm{~nm}$, and $(\mathrm{E} \& \mathrm{~F}) l_{s}=1.9 \mathrm{~nm}$ for isotropically in-plane (and radial orientation) dipoles on the left column (on the right column), where the distance of the dye-molecules from the surface of the silver sphere is $d=1 \mathrm{~nm}$ and the sphere radius $a=14 \mathrm{~nm}$ embedded in water $\varepsilon_{\mathrm{m}}=1.33^{2}$. The results from GCDM are shown as dash lines and the differential cross-section for the isotropically in-plane dipoles were multiplied by 25 . 
dipole moment of:

$$
\mathbf{p}_{\text {sphere }}=\alpha_{\text {sphere }} \mathbf{E}_{\text {Inc }}=4 \pi \varepsilon_{0} a^{3} \beta_{\text {sphere }} \mathbf{E}_{\text {Inc }},
$$

where $\alpha_{\text {sphere }}$ is the polarisability of the sphere, $a$ is the sphere's radius, $\beta_{\text {sphere }}=$ $\left(\varepsilon_{\text {sphere }}-1\right) /\left(\varepsilon_{\text {sphere }}+1\right)$, and $\varepsilon_{\text {sphere }}$ is the dielectric function of the sphere. At a point Q, distance $r$ away from the surface of that sphere, the electric field at that point $\mathbf{E}_{\mathrm{at} Q}$ is the sum of the incident field and the field created by that electric dipole p, i.e. $\mathbf{E}_{\text {at } \mathrm{Q}}=\mathbf{E}_{\mathrm{p}}+\mathbf{E}_{\text {Inc }}$. If we set the incident electric field along the $z$-direction $\mathbf{E}_{\mathrm{Inc}}=\mathrm{E}_{0} \hat{e}_{z}$, then

$$
\begin{aligned}
\mathbf{p}_{\text {sphere }} & =4 \pi \varepsilon_{0} a^{3} \beta_{\text {sphere }} \mathrm{E}_{0} \hat{e}_{z} \\
& =4 \pi \varepsilon_{0} a^{3} \beta_{\text {sphere }} \mathrm{E}_{0}\left(\cos \theta \hat{e}_{r}-\sin \theta \hat{e}_{\theta}\right) .
\end{aligned}
$$

Therefore,

$$
\begin{array}{r}
\mathbf{E}_{\text {at } \mathrm{Q}}=\left(\frac{2 \beta_{\text {sphere }} a^{2}}{(r+a)^{3}}+1\right) \mathrm{E}_{0} \cos \theta \hat{e}_{r}+\left(\frac{\beta_{\text {sphere }} a^{2}}{(r+a)^{3}}-1\right) \mathrm{E}_{0} \sin \theta \hat{e}_{\theta} \\
M_{\text {at Q }}^{\perp}=\frac{\left\langle\left|\mathbf{E}_{\text {at Q }}^{\perp}\right|^{2}\right\rangle}{\left|\mathbf{E}_{\text {Inc }}\right|^{2}}=\frac{1}{3}\left|\frac{2 \beta_{\text {sphere }} a^{3}}{(r+a)^{3}}+1\right|^{2} \\
M_{\text {at Q }}^{\|}=\frac{\left\langle\left|\mathbf{E}_{\text {at Q }}^{\|}\right|^{2}\right\rangle}{\left|\mathbf{E}_{\text {Inc }}\right|^{2}}=\frac{2}{3}\left|\frac{\beta_{\text {sphere }} a^{3}}{(r+a)^{3}}-1\right|^{2} .
\end{array}
$$

For $r \ll a$, we can do Binomial expansion for $(1+r / a)^{-3}$ and Eqs. 4.73 and 4.74 become:

$$
\begin{aligned}
& M_{\text {at } \mathrm{Q}}^{\perp} \approx \frac{1}{3}\left|1+2 \beta_{\text {sphere }}\left(1-\frac{3 r}{a}\right)\right|^{2} \approx \frac{1}{3}\left|1+2 \beta_{\text {sphere }}\right|^{2}\left|1-\frac{6 \beta_{\text {sphere }} r}{a\left(1+2 \beta_{\text {sphere }}\right)}\right|^{2} \\
& M_{\text {at } \mathrm{Q}}^{\|} \approx \frac{2}{3}\left|1-\beta_{\text {sphere }}\left(1-\frac{3 r}{a}\right)\right|^{2} \approx \frac{2}{3}\left|1-\beta_{\text {sphere }}\right|^{2}\left|1+\frac{3 \beta_{\text {sphere }} r}{a\left(1-\beta_{\text {sphere }}\right)}\right|^{2}
\end{aligned}
$$

At $\lambda=526 \mathrm{~nm}, \beta_{\text {sphere }}=1.381+i 0.0208$ (using $\varepsilon_{\text {silver }}=\operatorname{epsAg}(\lambda)$ in $\mathrm{SPlaC}$ ). When $\beta_{\text {sphere }} \rightarrow 1$, the factor $\left(1-\beta_{\text {sphere }}\right)$ in $M_{\text {at Q }}^{\|}$tends to converge to zero. Although this is a dipole approximation, it still can be used to explain the fact that the value of $M_{\text {at Q }}^{\|}$decays much faster than $M_{\text {at } \mathrm{Q}}^{\perp}$ as a function of distance away from the surface $d$, which is presented in Fig. 4.6. This explains the more pronounced $l_{s}$ dependence for the isotropically in-plane molecules, as evidenced in Fig. 4.5. 


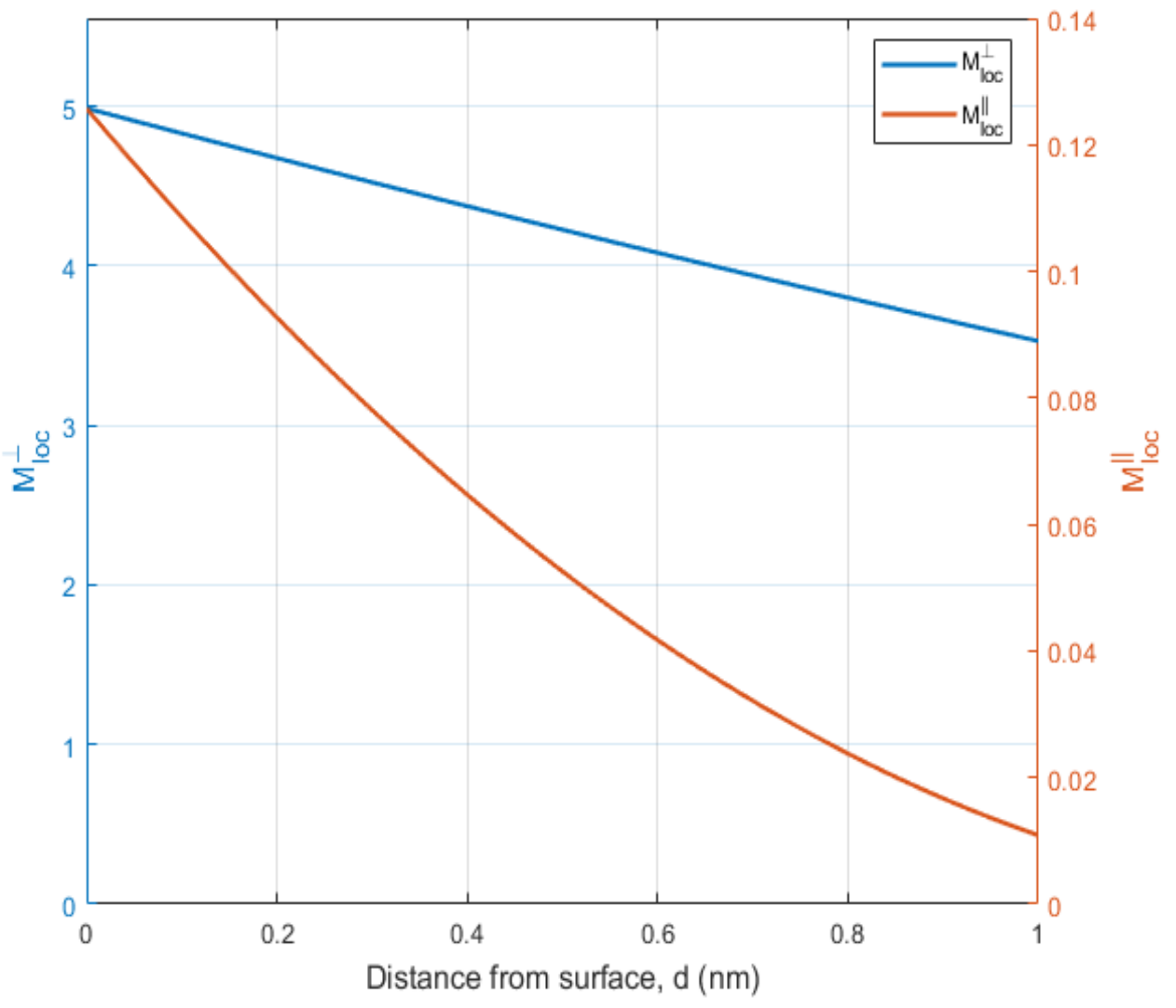

Figure 4.6: Surface-averaged field intensity enhancement $M_{\text {loc }}$ (perpendicular on the left axis and parallel on the right axis) at distance $d$ away from the surface of silver sphere of radius $a=14 \mathrm{~nm}$ at wavelength $\lambda=526 \mathrm{~nm}$. 


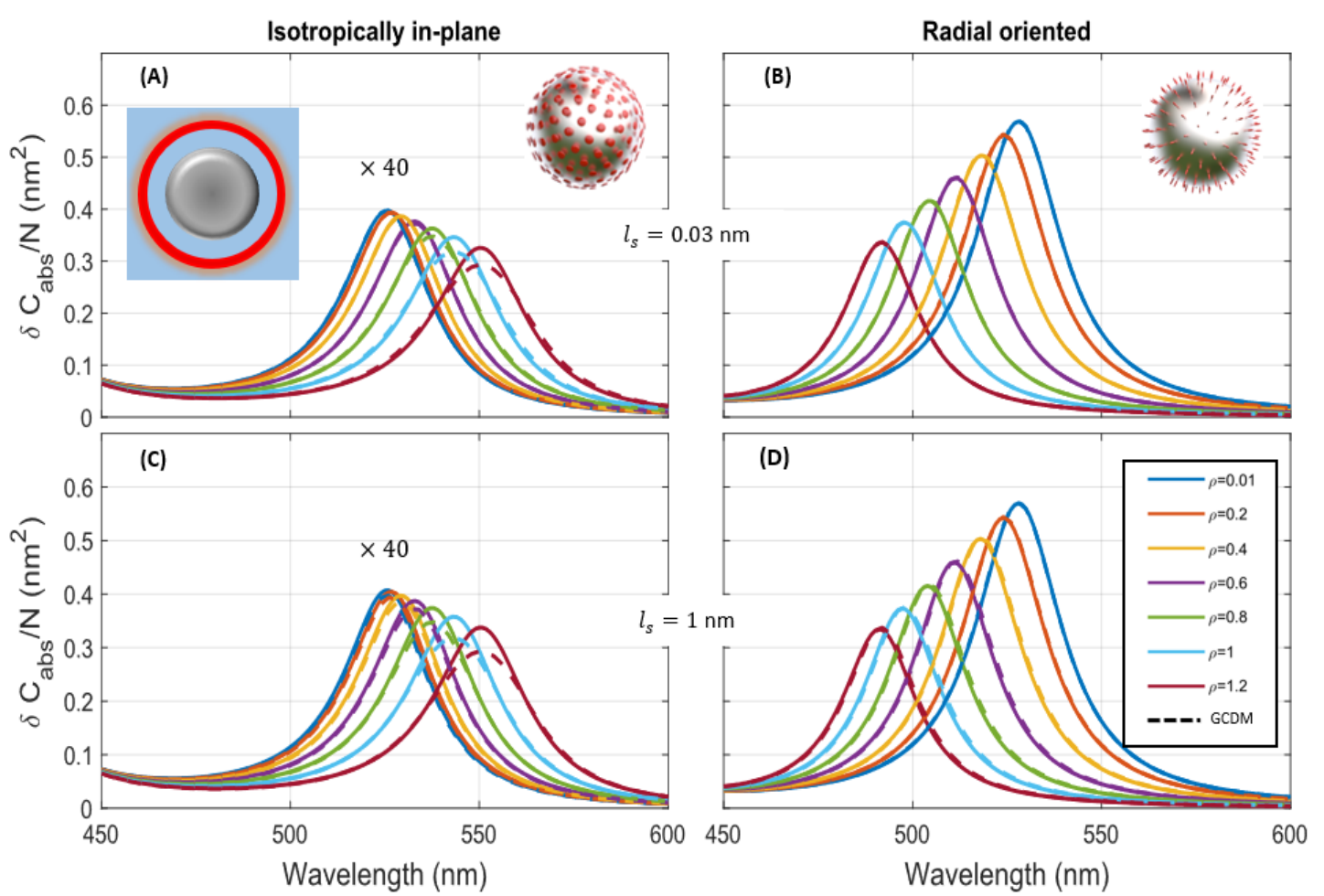

Figure 4.7: The effect of dye-concentration on the optical differential absorption crosssection using Mie theory with two-layer (solid line) with shell-thickness dependent (A \& B) $l_{s}=0.03 \mathrm{~nm}$ and $(\mathrm{C} \& \mathrm{D}) l_{s}=1 \mathrm{~nm}$ for isotropically in-plane (and radial orientation) dipoles on the left column (on the right column), where the distance of the dye-molecules from the surface of the silver sphere is $d=1 \mathrm{~nm}$ and the sphere radius $a=14 \mathrm{~nm}$ embedded in water $\varepsilon_{\mathrm{m}}=1.33^{2}$. The results from GCDM are shown as dash lines and the differential cross-section for the isotropically in-plane dipoles were multiplied by 40 . 


\subsubsection{Two-Layer System}

To overcome this $l_{s}$ dependency, a two-layer system is introduced (shown in the inset of Fig. 4.7 A and the TASA solutions presented in Appendix D), where the silver core and the anisotropic shell are separated by a water or air layer (with $\varepsilon_{\mathrm{m}}$ ) of thickness $d-l_{s} / 2$. This two-layer system adds another level of complexity to the single-layer solution, the more descriptive results can be found in our recent publication Ref. [74]. With this two-layer system, one can then define the distance from the dye molecules to the surface of the nanoparticle more appropriately compared to the geometry defined in GCDM. One can also choose the thickness $l_{s}$ to be very small and use the thin-anisotropic shell approximation to solve the problem as done for a singlelayer, which leads to the simplifications of the Bessel functions of complex orders. By using this two-layer system, the results show that by varying the thickness of the shell $l_{s}$ (as shown in Fig. 4.7 from $l_{s}=0.03 \mathrm{~nm}$ to $l_{s}=1 \mathrm{~nm}$ ), the absorption cross-sections for both cases do not change as much compared to a single layer system. Furthermore, from Fig. 4.7, we can also conclude that the results from EMM predictions are in great agreement with GCDM for the smaller value of $l_{s}$, i.e. thin shell. The discrepancies in the isotropically in-plane case at higher coverage are due to small curvature effects, which should not be a problem for larger spheres.

\subsection{Self-Reflected Field or Image-Dipole Effects}

In all of the above cases, the distance $d$ from the dipole position to the metal surface was set to be $1 \mathrm{~nm}$ in the GCDM calculations, so that the effect of the image dipoles are negligible. However, we cannot ignore this effect when the dye molecules are adsorbed very close to the surface of the metallic nanoparticle. When a polarisable dipole is located close to a dielectric or metallic surface/object, the electromagnetic field reflected (or scattered) by the object can affect its optical properties [27, 73]. For this self-reflected field or image-dipole effect to be properly accounted, we can start from the classical image theory of electromagnetism. In the first approximation for sufficiently small distance $d$, the surface may be considered to be planar and the retardation effects may be safely neglected, which results in a simple electrostatic problem. For a dipole $\mathbf{p}$ in a medium located at $\mathbf{r}_{n}=\left(x_{n}, y_{n}, d\right)$ above a metal surface with dielectric function $\varepsilon_{\text {in }}$ will induce an image dipole:

$$
\mathbf{p}_{I}=\beta_{\mathrm{m}}\left(-\mathrm{p}_{x},-\mathrm{p}_{y}, \mathrm{p}_{z}\right),
$$

located $\mathbf{r}_{I}=\left(x_{n}, y_{n},-d\right)$, where $\beta_{\mathrm{m}}=\left(\varepsilon_{\mathrm{in}}-\varepsilon_{\mathrm{m}}\right) /\left(\varepsilon_{\mathrm{in}}+\varepsilon_{\mathrm{m}}\right)$. This image-dipole $\mathbf{p}_{I}$ will then induce an electric field $\mathbf{E}_{\text {dip, } I}$ which then contribute to the local field $\mathbf{E}_{\text {loc }}$ 
at the dipole position.

\subsubsection{View from Microscopic Model: Effective Polarisability with Image-Dipole Effect}

For simplicity, we shall first look at the effect from the image of a single polarisable dipole on a metal sphere. We will here begin with the microscopic model as done in the previous chapter (section 3.3.1). We only consider the dilute case here, i.e. a low concentration of dye only. Without accounting for the image-dipole effect, the absorption cross-section for a single dipole on a sphere is given by Eq. 3.62. To account for the image dipole effect, we will need to re-calculate the new local field $\mathbf{E}_{\mathrm{loc}, I}$ ( the correction to $\mathbf{E}_{\mathrm{loc}}$ with the contribution from $\mathbf{p}_{I}$ ), or define an effective polarisability $\left(\alpha_{\text {eff }}\right)$ which includes the effect of image dipole.

$$
\begin{aligned}
\mathbf{p} & =\alpha_{z z}\left[L_{\mathrm{m}}\left(\mathbf{E}_{\mathrm{loc}}+L_{\mathrm{m}} \mathbf{E}_{\mathrm{dip}, I}\right) \cdot \hat{e}_{\mathrm{d}}\right] \hat{e}_{\mathrm{d}} \\
& =\alpha_{\mathrm{eff}}\left[L_{\mathrm{m}} \mathbf{E}_{\mathrm{loc}} \cdot \hat{e}_{\mathrm{d}}\right] \hat{e}_{\mathrm{d}} .
\end{aligned}
$$

For the two different dipole orientations, the effective polarisability can be found as:

$$
\begin{aligned}
& \alpha_{\mathrm{eff}}^{\|}=\frac{\alpha_{z z} / 2}{1-\frac{\beta_{\mathrm{m}} \alpha_{z z}}{4 \pi \varepsilon_{0}(2 d)^{3}} \frac{L_{\mathrm{m}}^{2}}{2 \varepsilon_{\mathrm{m}}}}, \\
& \alpha_{\mathrm{eff}}^{\perp}=\frac{\alpha_{z z}}{1-\frac{2 \beta_{\mathrm{m}} \alpha_{z z}}{4 \pi \varepsilon_{0}(2 d)^{3}} \frac{L_{\mathrm{m}}^{2}}{\varepsilon_{\mathrm{m}}}} .
\end{aligned}
$$

Hence, by taking into account the image-dipole effect, Eq. 2.43 will become:

$$
\begin{aligned}
\sigma_{\mathrm{abs}, I} & =\frac{2 \pi}{\lambda \sqrt{\varepsilon_{\mathrm{m}}}} L_{\mathrm{m}}^{2} \frac{\left|\hat{e}_{\mathrm{d}} \cdot \mathbf{E}_{\mathrm{loc}, I}\right|^{2}}{\left|\mathbf{E}_{0}\right|^{2}} \frac{\Im\left(\alpha_{z z}\right)}{\varepsilon_{0}}, \\
\text { or } \quad \sigma_{\mathrm{abs}, I} & =\frac{2 \pi}{\lambda \sqrt{\varepsilon_{\mathrm{m}}}} L_{\mathrm{m}}^{2} \frac{\left|\hat{e}_{\mathrm{d}} \cdot \mathbf{E}_{\mathrm{loc}}\right|^{2}}{\left|\mathbf{E}_{0}\right|^{2}} \frac{\Im\left(\alpha_{z z}\right)}{\varepsilon_{0}}\left|\frac{\alpha_{\mathrm{eff}}}{\alpha_{z z}}\right|^{2} .
\end{aligned}
$$

And as for the dielectric functions, if we were to replace $\alpha$ (in Eqs. 4.24 and 4.26) with the effective polarisabilities defined in Eqs. 4.78 and 4.79 (for low $\rho$ ), we get:

$$
\begin{aligned}
& \varepsilon_{x y}=\varepsilon_{\mathrm{m}}+\frac{L_{\mathrm{m}}^{2} c_{\mathrm{d}} \alpha_{z z} /\left(2 \varepsilon_{0}\right)}{1-\frac{\alpha_{z z}}{4 \pi \varepsilon_{0}} \frac{1}{2} \frac{L_{\mathrm{m}}^{2}}{\varepsilon_{\mathrm{m}}} \frac{\beta_{\mathrm{m}}}{(2 d)^{3}}}, \\
& \frac{1}{\varepsilon_{z z}}=\frac{1}{\varepsilon_{\mathrm{m}}}-\frac{\left(L_{\mathrm{m}} / \varepsilon_{\mathrm{m}}\right)^{2} c_{\mathrm{d}} \alpha_{z z} / \varepsilon_{0}}{1-\frac{\alpha_{z z}}{4 \pi \varepsilon_{0}} \frac{L_{\mathrm{m}}^{2}}{\varepsilon_{\mathrm{m}}} \frac{2 \beta_{\mathrm{m}}}{(2 d)^{3}}} .
\end{aligned}
$$

This indicates that, at a very low dye-concentration, the main contribution to the local field correction is from the image-dipole effect only. 


\subsubsection{Anisotropic EMM with Image-Dipole Effect}

At high concentration, Eqs. 4.82 and 4.83 will no longer work since those have not accounted for the effect of the image of the other dipoles into the local field correction. Previous studies had been done to find an effective dielectric functions for planar arrays of dipoles near a substrate [45, 46], which accounted for orientation effect, dye-concentration and image dipole effects properly. The effective dielectric functions of the medium strongly depend on the dipoles' orientations, either isotropically in-plane or normal to the surface of the metal substrate. The full expressions for these are shown in Eqs. 3.12 and 3.13 of Ref. [45]. However, these previous studies were applied only to planar array adsorbed on planar films in vacuum, similar to the work done by Dignam and Moskovits [44]. We here will generalise the results obtained by Bagchi et al. $[45,46]$ to the case when the system is embedded in a medium with dielectric permittivity $\varepsilon_{\mathrm{m}} \neq 1$. This will be more relevant to many experimental conditions where particles are suspended in water or other solvents.

In doing so, similar procedure (as done in section 3.1.2 C-D) can be done to account for the image-dipole effect. We assume that dipoles (with dipoles moment p) separated from the surface of the metallic sphere by a distance $d$ will induce an image dipole $\mathbf{p}_{I}=\beta_{\mathrm{m}}\left(-\mathrm{p}_{x},-\mathrm{p}_{y}, \mathrm{p}_{z}\right)$. These image-dipoles will then induce macroscopic field $\mathbf{E}_{\mathrm{dip}, I}$ which contributes to the local field $\mathbf{E}_{\mathrm{loc}}$ at a chosen dipole's position. So, the dipole moment in Eq. 4.21 can now be re-expressed as:

$$
\mathbf{p}(\mathbf{0})=\underline{\alpha}_{\mathrm{d}} L_{\mathrm{m}}\left[\mathbf{E}_{\mathrm{Ext}}+\frac{L_{\mathrm{m}}}{\varepsilon_{\mathrm{m}}}\left(\sum_{\mathbf{r}_{n} \neq \mathbf{0}} \mathbf{E}_{\mathrm{dip}}\left(\mathbf{p}, \mathbf{r}_{n}\right)+\sum_{\mathbf{r}_{I}} \mathbf{E}_{\mathrm{dip}, I}\left(\mathbf{p}_{I}, \mathbf{r}_{I}\right)\right)\right] .
$$

Following the boundary conditions and the definition of the dielectric displacement, the modified effective dielectric functions can be derived to be:

$$
\begin{aligned}
& \varepsilon_{x y, I}=\varepsilon_{\mathrm{m}}+\frac{L_{\mathrm{m}}^{2} c_{\mathrm{d}} \alpha / \varepsilon_{0}}{1-\frac{\alpha}{4 \pi \varepsilon_{0}} \frac{L_{\mathrm{m}}^{2}}{\varepsilon_{\mathrm{m}}} \rho^{3 / 2}\left(\xi_{0}+\beta_{\mathrm{m}} \xi_{I}\right) / 2}, \\
& \frac{1}{\varepsilon_{z z, I}}=\frac{1}{\varepsilon_{\mathrm{m}}}-\frac{\left(L_{\mathrm{m}}^{2} / \varepsilon_{\mathrm{m}}^{2}\right) c_{\mathrm{d}} \alpha / \varepsilon_{0}}{1+\frac{\alpha}{4 \pi \varepsilon_{0}} \frac{L_{\mathrm{m}}^{2}}{\varepsilon_{\mathrm{m}}} \rho^{3 / 2}\left(\xi_{0}-\beta_{\mathrm{m}} \xi_{I}\right)},
\end{aligned}
$$

where $\xi_{I}$ is akin to a lattice sum which depends on $d$ and $\rho$ and can be computed more efficiently as done in Ref. [46]:

$$
\begin{aligned}
\xi_{I} & =\sum_{i, j=-\infty}^{\infty} \frac{8 \rho d^{2}-\left(i^{2}+j^{2}\right)}{\left[i^{2}+j^{2}+4 \rho d^{2}\right]^{5 / 2}} \\
& =16 \pi^{2} \sum_{i=0}^{\infty} \sum_{j=1}^{\infty} \sqrt{i^{2}+j^{2}} e^{-4 \pi d \sqrt{\rho} \sqrt{i^{2}+j^{2}}}
\end{aligned}
$$




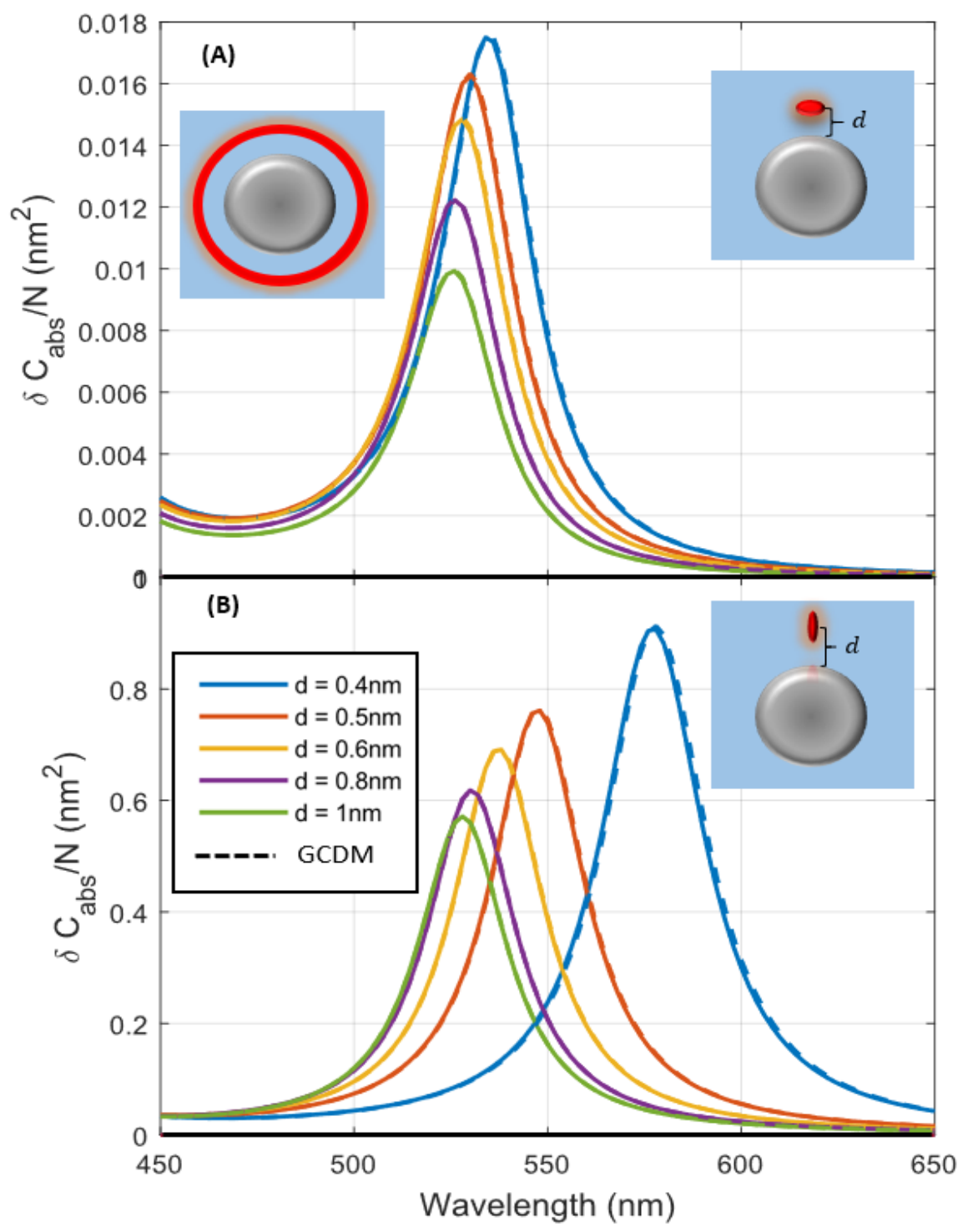

Figure 4.8: The effect of image-dipole on the optical differential absorption cross-section using Mie theory with 2 layers (solid line) with $d$-dependent (A) for isotropically in-plane dipoles and (B) for radially oriented dipoles where the dye coverage is chosen to be $\rho=0.001 \mathrm{~nm}^{-2}$, the thickness $l_{s}=0.03 \mathrm{~nm}$, and the sphere radius $a=14 \mathrm{~nm}$ embedded in water $\varepsilon_{\mathrm{m}}=1.33^{2}$. These results are compared to those from GCDM in dash lines. 


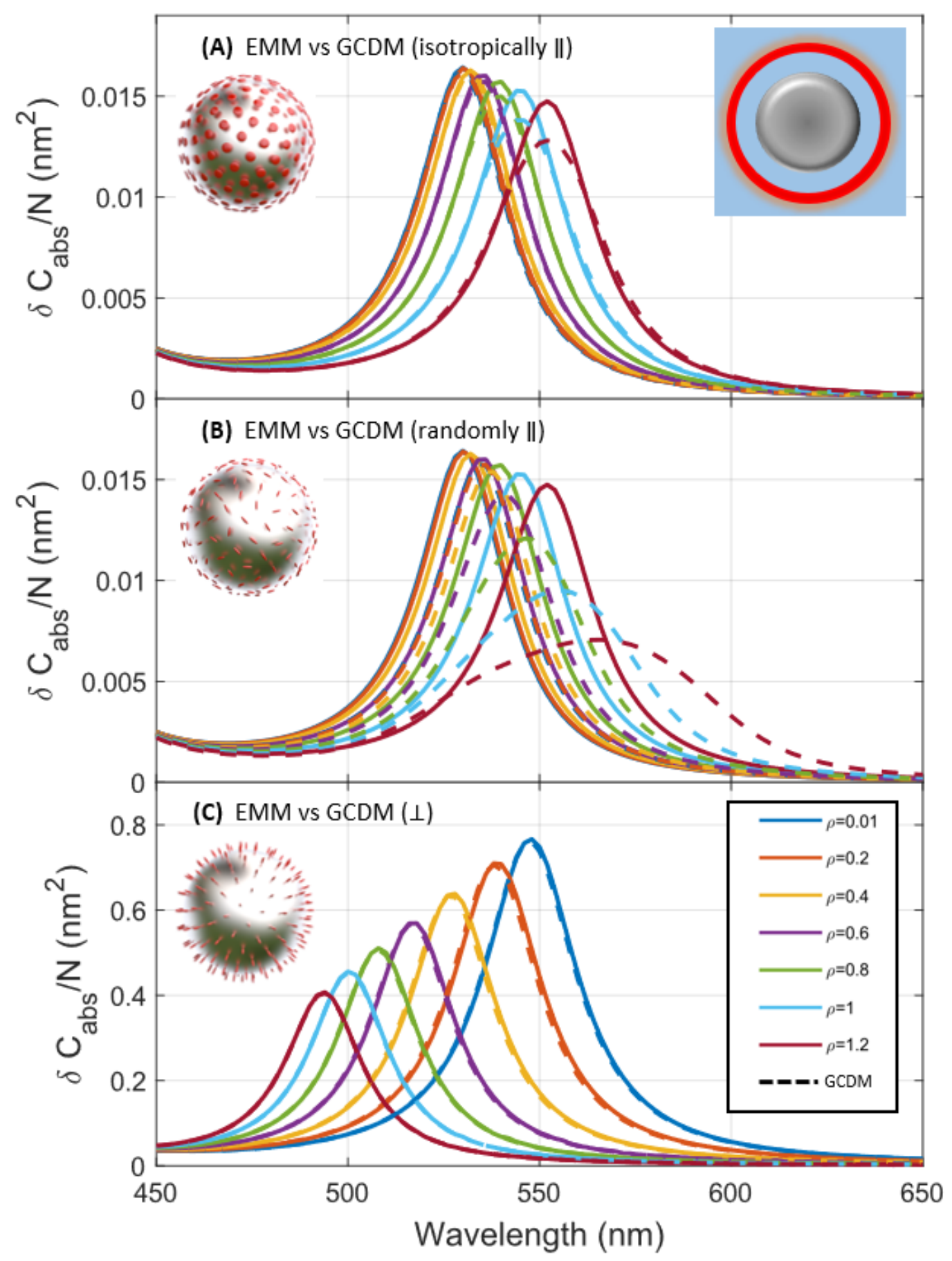

Figure 4.9: Comparison between the 2 layers EMM model and GCDM at a dipole distance $d=0.5 \mathrm{~nm}$ where the image-dipole effects become important, as a function of dyecoverage for (A) isotropically in-plane, (B) randomly oriented in-plane, and (C) radially oriented dipoles. We use $l_{s}=0.03 \mathrm{~nm}$ and the separate between the shell and the core is $d-l_{s} / 2$. 
At low dye-concentration, Eqs. 4.85 and 4.86 will reduce to the same expressions as in Eqs. 4.82 and 4.83. And if the dipoles are in vacuum, i.e. $\varepsilon_{\mathrm{m}}=1$, then they will simplify to the exact same expressions as (Eqs. 3.12 and 3.13) in Ref. [45].

We have just shown that the effective shell model (modified effective dielectric functions with anisotropic Mie theory) can account for multiple interactions: molecular orientation effect, non-vacuum embedding medium, dipole-dipole interactions, as well as the image-dipole effects (important at small separation from the surface). We shall now compare in Fig. 4.8 (using two-layer system) the results from GCDM and Mie theory with modified effective DF (Eqs. 4.85 and 4.86). We here set a low dye coverage of $\rho=0.001 \mathrm{~nm}^{2}$ which is a close approximation for a single dipole near a sphere in GCDM simulation. The effective shell model predicts the correct red-shift in absorption spectra as a function of $d$ for each orientation. The distance $d$ is significant here as it determines the strength of the image-dipole effect.

\subsubsection{A Complete EMM vs GCDM}

We show in Fig. 4.9 the effect of coverage for a fixed distance $d=0.5 \mathrm{~nm}$ from the anisotropic shell to the silver nanoparticle. This distance is small enough to capture the image-dipole effect, and the additional effect of the dipole-dipole interactions is also expected, including the interactions mediated by the nanoparticle, GCDM [37]. This is yet one of the most complex cases, and as presented in Fig. 4.9, the complete EMM performs very well. It shows for the perpendicular orientation (Fig. $4.9 \mathrm{C}$ ), the agreement is extremely good compared with GCDM. For the isotropically in-plane orientation (shown in Fig. 4.9A), the EMM predicts the correct shifts in the dye-resonance, but there are discrepancies in the intensities. The cause for these discrepancies is the contribution from a small curvature effect of the nanoparticle. This should become less prominent for larger spheres. We have tested this by looking at a smaller sphere with radius of $7 \mathrm{~nm}$, and the discrepancies in the intensities have become worse. We also compare in Fig. 4.9B, the EMM predictions to those from the GCDM for randomly oriented in-plane dipoles. The discrepancies are larger, which is because the EMM does not account for the random in-plane orientation and instead, it replaces all dipoles by isotropically in-plane polarisable smarties-like dipoles. Therefore, the information about the dipoles pointing in different directions is lost, since the interactions between two uniaxial dipoles in random directions is different from the interactions between two isotropic dipoles. In this case (for randomly in-plane dipoles), GCDM provides more realistic descriptions of the problem. The GCDM can account for the orientational disorder, which results 
in the broadening of the absorption peak, as we observed.

\subsection{Conclusion}

In summary, we have introduced a refined effective medium model for molecular monolayers on a metallic surface that accounts for: a non-vacuum embedding medium, coverage dependence, molecular orientations effects, and self-reflected field or image-dipole effects. All these effects can be used to elucidate relevant experimental results, for example, dye-on-nanoparticle. These effective DF were then used to implement into the anisotropic Mie theory for a two-layer system and compared to a more realistic model (GCDM) to show their validity and applicability. Good agreements between the two models were evidenced. We believe that these effective DF with the two-layer anisotropic Mie theory will find its application in many areas such as: in molecular plasmonics (including both weak and strong resonance couplings), and surface-enhanced spectroscopy, where the orientation and the coverage of the adsorbates result in anisotropic and self-reflected field responses and become to have larger effects. Furthermore, this work can be useful as a tool to facilitate the disentanglement of various mechanisms at play in strong coupling experiments from intrinsic shifts in the electronic resonance (via dye-dye interactions) to plasmon-dye interactions.

In the GCDM, the image-dipole effect is evidenced when a dipole is located close enough to the surface of the dielectric sphere with a high refractive index. To account for the dipole-sphere interactions, GCDM requires very high order multipoles, i.e. $N_{\max }=500$. However, for the effective shell model (without image-dipole corrections in the polarisability or DF), increasing the order of multipoles, the effect of image-dipole cannot be predicted. Nevertheless, if the image-dipole effects are accounted for in the effective DF, one could check that the results are, in fact, converged when including only $N_{\max }=1$ (the dipole terms). We can therefore use a relatively small number $N_{\max }=5$ of mutipoles for all our anisotropic Mie theory calculations.

As we know, Mie theory can only provide solutions for the light scattering problem by spherical objects in spherical geometry. However, we also want to look at how the change in shapes (for examples spheroidal particles or spheroidal coated particles) can affect the outcome of the optical properties compared to those of spherical objects. Therefore, in chapter 5 , we will seek the advantages of COMSOL simula- 
tions to extend our studies from spherical to spheroidal objects, which includes the anisotropic responses in the shell. Also, how the dye layer may affect the shift in plasmon resonance of the nanoparticles will be investigated in Chapter 6 . 


\section{Anisotropic EMM Shells on Non-Spherical Particles}

The light scattering problem by core/shell systems (coated nanoparticles) has been our main focus throughout this thesis, especially for spherical nanoparticles. Many studies have also been done on light scattering by non-spherical particles, e.g. spheroids [36], and nanorods [23]. However, the studies of when these non-spherical particles surrounded by anisotropic molecules have not been properly investigated, nor welldocumented. Therefore, in this chapter, we will extend our work in the previous chapters to non-spherical coated nanoparticles, i.e. spheroidal core/shell system.

Spheroidal geometry has been used to investigate and study the light scattering problem by elongated (or flatten) nanoparticles where highly localised regions of intense electric field enhancements occur at the tip(s), so-called electromagnetic hotspots. For non-spherical objects, it is almost the same for all other light scattering problems, where one needs to seek solutions to the Maxwell equations. Although we know that Mie theory [38] and/or T-matrix method [49] (which are the analytic and semi-analytic solutions, respectively) are ideal, these are limited to specific types of scatterers. Instead, we try to produce efficient and accurate numerical solutions, which have been previously studied $[75,76,77,78]$. There are different techniques which can be used to study spheroids, for example, fully numerical partial differential equation solvers such as finite-difference time-domain (FDTD) [51] and the finite element method (FEM) [50]. Recently, the practical implementation of the FEM calculations in COMSOL ${ }^{1}$ was done in Ref. [79]. This work provided full details of how to set up calculations in COMSOL, which were applied to spherical nanoparticles, dimer consisting of two spherical nanoparticles, and prolate spheroidal nanoparticles.

It is in our interest to use the practical implementation of the FEM calculation

\footnotetext{
${ }^{1}$ COMSOL: a commercial software which is a cross-platform finite-element analysis. It allowed users to solve interdependent physics problems within the same domain.
} 
in COMSOL (especially for anisotropic coated spheroidal particle) because: 1) Mie theory cannot provide solutions for particles with non-spherical geometry, and 2) extending the GCDM with $T$-matrix framework (for spheroids) would more likely run into numerical problems at high multipole orders and fundamental problems related to the Rayleigh hypothesis, which is non-trivial and rather complicated (even for the isotropic core-shell system). Hence, they are not the preferred methods to use. However, with the capability of COMSOL in solving multi-physics problems, we will be able to extend the study in Ref. [79] into coated nanoparticles with anisotropic responses in the coating layer for both spherical and spheroidal nanoparticles. Although COMSOL simulation time is taking much longer than Mie theory (approximately of the similar time-scale as the GCDM), the main advantage is that it can be used to study light scattering problem by non-spherical objects. This chapter will demonstrate how anisotropic effects in the shell can be accounted for in the COMSOL implementation for these geometries. Firstly, we will use FEM implementation in COMSOL to reproduce the results for spherical silver and gold nanoparticles and check these against the analytical (semi-analytical) solutions, Mie theory (T-matrix) for spheres (spheroids). In doing so, we shall look at what has been done in Ref. [79].

\subsection{General Considerations in COMSOL}

\subsubsection{FEM Model Description}

The finite-element method (FEM) [50] involves solving the Helmholtz equation (or Maxwell's equations) in frequency domain whereby the spatial derivatives at the surface of the scatterer are solved numerically as a boundary value problem to find electric and magnetic fields that satisfy the boundary conditions ${ }^{2}$. The advantages of FEM is that it can also be used to model the light scattering by arbitrary shaped, dispersive, inhomogeneous, and anisotropic structures for a single frequency at a time. The grid mesh (triangular or tetrahedral) is used to discretise surfaces or the overall space into many smaller areas or regions. The mesh can be chosen to be denser for regions with fine structure. Users can define a suitable mesh to ensure that the object(s) is represented with a sufficiently accurate mesh.

The important point in the FEM approach is to avoid the reflection of the inci-

\footnotetext{
${ }^{2}$ (i) The tangential component of the fields are continuous across the particle's surface, and (ii) for large value of distance $r$, the far-field decays no slower than $1 / r$.
} 
dent or scattered fields at the boundary of the computational domain, which could result in an inaccurate solution for the problem. Therefore, a bounding box with appropriate physical conditions needs to be defined. Perfectly matched layers (PML) is one of the most efficient approaches to be chosen as a bounding box. Users have to ensure adequate meshing for this region. As described in Ref. [79], PML setting can be found in COMSOL as a ready-to-use setting and more descriptions of the setting for the meshing can be found in its supplementary information.

\subsubsection{Electric Fields}

We here consider the standard problem of electromagnetic scattering by a particle, as in Ref. [49]. The time dependence, $e^{+i \omega t}$, is chosen as the complex notations for the EM fields ${ }^{3}$. The particle is embedded in a non-absorbing medium of relative dielectric constant $\varepsilon_{\mathrm{m}}$. It is excited at a wavelength $\lambda$ by a plane wave incident field $\mathbf{E}_{\text {Inc }}(\mathbf{r})$ of amplitude $\mathrm{E}_{0}$ along $z$ and polarised along $x$. The wave-vector, $k_{\mathrm{m}}$, and the incident field, $\mathbf{E}_{\text {Inc }}$ are defined as:

$$
\begin{gathered}
k_{\mathrm{m}}=\frac{2 \pi \sqrt{\varepsilon_{\mathrm{m}}}}{\lambda}, \\
\mathbf{E}_{\mathrm{Inc}}(\mathbf{r})=\mathrm{E}_{0} \exp \left(-i k_{\mathrm{m}} z\right) \hat{\mathbf{e}}_{x} .
\end{gathered}
$$

For simplicity in COMSOL, we can write the solution of the field as $\mathbf{E}(\mathbf{r})=\mathbf{E}_{\text {Inc }}(\mathbf{r})+$ $\mathbf{E}_{\mathrm{Sca}}(\mathbf{r})$, where $\mathbf{E}_{\mathrm{Sca}}(\mathbf{r})$ denotes the scattered field (same notation as in chapter 1). Next, we will define useful quantities that we are interested in and compare them to the exact solutions (Mie theory).

\subsubsection{Absorption, Scattering, and Extinction Cross-Sections}

The absorption cross-section is calculated by integrating the total power dissipated density $Q_{h}=-\omega \Im\left\{\varepsilon_{\text {in }}(\omega)\right\}|\mathbf{E}(\mathbf{r})|^{2} / 2$, [where $\varepsilon_{\text {in }}(\omega)$ denotes the dielectric function of the particle] over the volume inside the particle:

$$
\sigma_{\mathrm{Abs}}=\frac{1}{S_{0}} \iiint_{\mathrm{NP}} Q_{h} d V_{\mathrm{NP}}
$$

where $S_{0}=\sqrt{\varepsilon_{\mathrm{m}}} \mathrm{E}_{0}^{2} /\left(2 Z_{0}\right)$ is the power density of the incident field and $Z_{0}=$ $\sqrt{\mu_{0} / \varepsilon_{0}} \approx 376.73 \Omega$ is the characteristic impedance of vacuum. Equation 5.3 is equivalent to Eq. 2.40 with absorption power given in Eq. 2.36, which can be proved by using the Divergence theorem.

\footnotetext{
${ }^{3}$ This is different from the commonly used convention in physics and in the rest of this thesis but is the one chosen in the COMSOL calculations.
} 
The scattering cross-section can be calculated by integrating the flux of the complex Poynting vector of the scattered field $\left(\mathbf{S}_{\text {Sca }}=1 / 2\left[\mathbf{E}_{\text {Sca }} \times \mathbf{H}_{\text {Sca }}^{*}\right]\right)$ across the nanoparticle surface:

$$
\sigma_{\mathrm{Sca}}=\frac{1}{S_{0}} \iint_{\mathrm{NP}} \Re\left\{\mathbf{S}_{\mathrm{Sca}} \cdot \hat{\mathbf{n}}\right\} d S_{\mathrm{NP}}
$$

Again, this is equivalent to the scattering cross-section defined in Eq. 2.40 with Eq. 2.35. The extinction cross-section can be calculated from the conservation of energy as, $\sigma_{\mathrm{Ext}}=\sigma_{\mathrm{Abs}}+\sigma_{\mathrm{Sca}}$. Although other quantities can be calculated by the COMSOL simulation (as described in Ref. [79]), we will leave them aside since we are only interested in the cross-sections.

\subsubsection{Single Spherical Particle}

It is reasonable to consider a spherical particle first, since we can use Mie theory, which provides an exact analytical solution to the scattering problem by a sphere [38], to check against the solution from COMSOL numerical simulations. This is an important system in many types of experiments, for examples, using gold nanoparticles of different shapes (nanospheres, nanorods, nanoshells) to study the modalities of cancer therapy (using plasmonic photothermal therapy, PPTT) [80], the techniques of using UV-vis spectra to determine the size and concentration of nanoparticles [81], and the investigation of modified optical absorption of molecules on metallic nanoparticles at sub-monolayer coverage [34] along with the theoretical studies $[35,48,37]$. The light scattering problem by a single gold spherical nanoparticle has been simulated by COMSOL as done in Ref. [79]. This will be used as our first step toward the simulations and study for the light scattering problem by coated particles with different shapes. Without loss of generality, we will revisit it here and include a silver sphere and a gold sphere, with the size of $30 \mathrm{~nm}$ in radius and embedded in water instead, i.e. $\varepsilon_{\mathrm{m}}=1.33^{2}$, as it is more relevant to most experiments. The results from the COMSOL simulations will then be compared with Mie theory. For all of the results from COMSOL simulations below, 5-layer spherical PML will be used, as it was pointed out to be a better choice in Ref. [79]. 


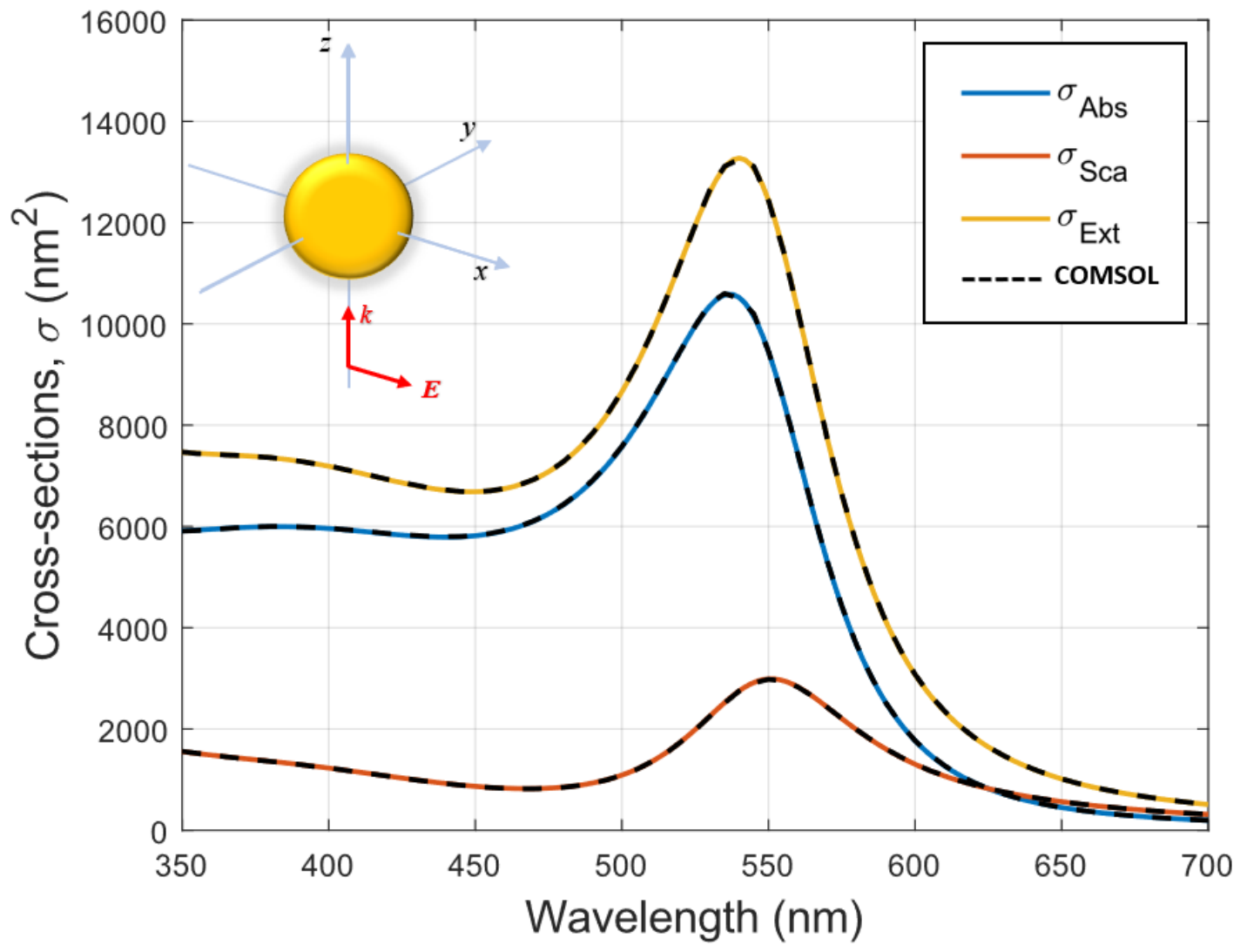

Figure 5.1: Comparison between FEM (COMSOL simulations) and Mie theory results for a $30 \mathrm{~nm}$ radius gold sphere embedded in water $\varepsilon_{\mathrm{m}}=1.33^{2}$. The results show the wavelength dependence of the absorption, scattering, and extinction cross-sections from Mie theory (colour lines) and COMSOL (dash lines). 


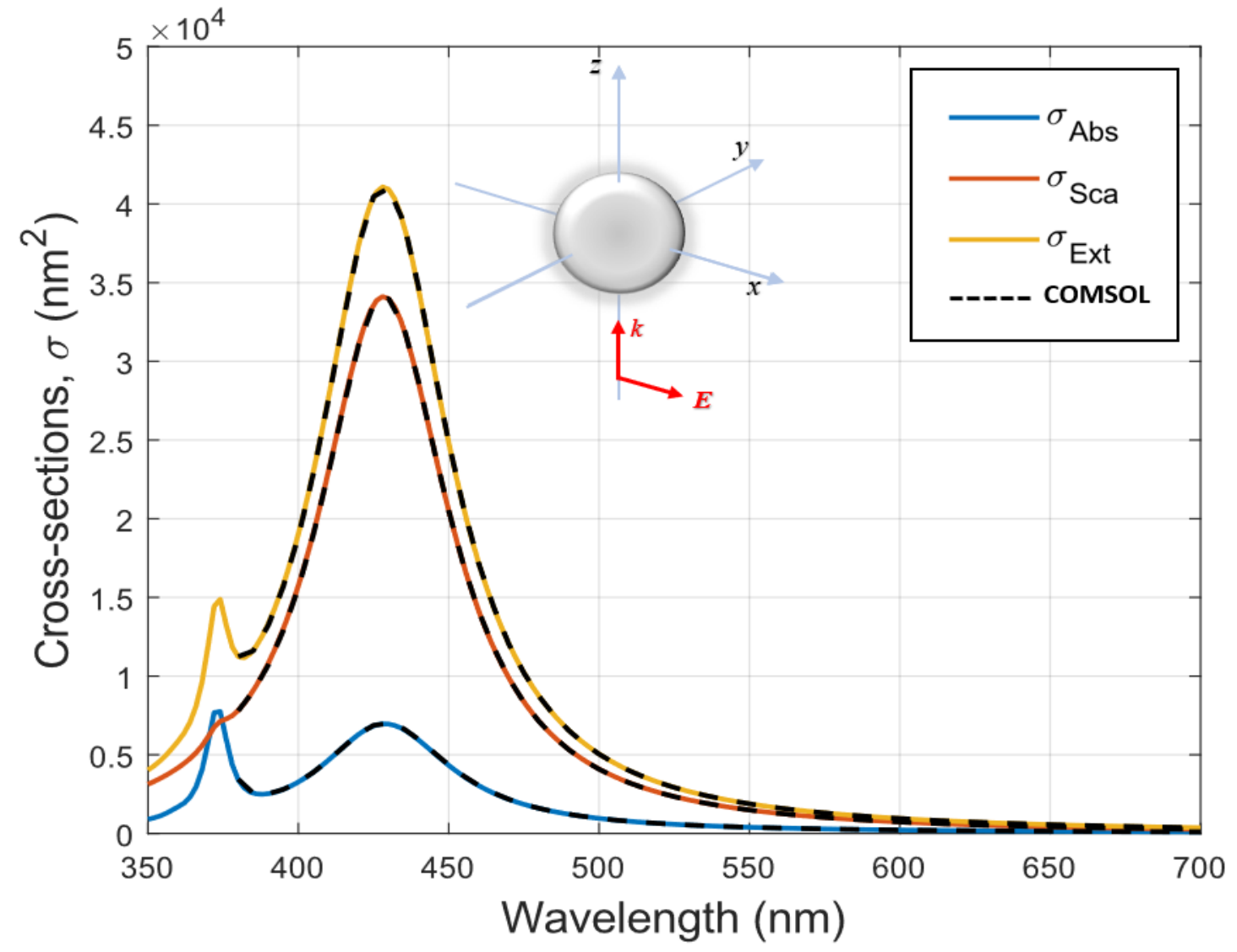

Figure 5.2: Comparison between FEM (COMSOL simulations) and Mie theory results for a $30 \mathrm{~nm}$ radius silver sphere embedded in water $\varepsilon_{\mathrm{m}}=1.33^{2}$. The results show the wavelength dependence of the absorption, scattering, and extinction cross-sections from Mie theory (colour lines) and COMSOL (dash lines). 
The dielectric functions of gold and silver used in the simulations were given in Ref. [82] as:

$$
\begin{aligned}
& \varepsilon_{\mathrm{Au}}(\lambda)=\varepsilon_{\infty}\left[1-\frac{1}{\lambda_{p}^{2}\left(\frac{1}{\lambda^{2}}+\frac{i}{\mu_{p} \lambda}\right)}\right] \\
& +\sum_{n=1,2} \frac{A_{n}}{\lambda_{n}}\left[\frac{e^{i \phi_{n}}}{\frac{1}{\lambda_{n}}-\frac{1}{\lambda}-\frac{i}{\mu_{n}}}+\frac{e^{-i \phi_{n}}}{\frac{1}{\lambda_{n}}+\frac{1}{\lambda}+\frac{i}{\mu_{n}}}\right] \text {, } \\
& \text { where } \quad \varepsilon_{\infty}=1.54, \quad \lambda_{p}=177.5 \mathrm{~nm}, \quad \mu_{p}=14500 \mathrm{~nm} \text {, } \\
& A_{1}=1.27, \quad \phi_{1}=-\pi / 4, \quad \lambda_{1}=470 \mathrm{~nm}, \quad \mu_{1}=1900 \mathrm{~nm}, \\
& A_{2}=1.10, \quad \phi_{2}=-\pi / 4, \quad \lambda_{2}=325 \mathrm{~nm}, \quad \mu_{2}=1060 \mathrm{~nm} . \\
& \varepsilon_{\mathrm{Ag}}(\lambda)=\varepsilon_{\infty}\left[1-\frac{1}{\lambda_{p}^{2}\left(\frac{1}{\lambda^{2}}+\frac{i}{\mu_{p} \lambda}\right)}\right], \\
& \text { where } \quad \varepsilon_{\infty}=4, \quad \lambda_{p}=282 \mathrm{~nm}, \quad \mu_{p}=17000 \mathrm{~nm} .
\end{aligned}
$$

NB: for the implementation of these dielectric functions (of gold and silver) in COMSOL, we need to take their complex conjugates, as the time dependence of the EM fields was chosen as $e^{+i \omega t}$.

For the particle of radius $30 \mathrm{~nm}$, the inner-radius of the PML is chosen to be $250 \mathrm{~nm}$ from the centre of the particle and its thickness was set to be $100 \mathrm{~nm}$. The electric field of the plane wave incident is chosen along the $x$-axis with the $\mathbf{k}$-vector along the $z$-axis. For the configuration of this problem, the results of the crosssections should not depend on the direction of the plane-wave incident. With the same grid mesh as chosen in Ref. [79], the cross-sections' spectra can be computed for both gold and silver spherical particles, and then compared with Mie theory as shown in Figs. 5.1 and 5.2, for gold and silver, respectively. The relative errors in the discrepancies between the COMSOL simulations and Mie theory are almost indistinguishable, in the order of $\sim 0.4 \%$ for gold and $\sim 1 \%$ for silver. So, we can conclude that both of them are in agreement (at least for $\lambda>380 \mathrm{~nm}$ for the silver sphere).

For $\lambda<380 \mathrm{~nm}$ the solution from COMSOL for silver sphere does not converge as the real part of the dielectric function is close to $-3 / 2$ of the dielectric constant of the 
medium, equivalently $\Re\left\{\varepsilon_{\mathrm{Ag}}\right\} \rightarrow-(3 / 2) \varepsilon_{\mathrm{m}}$, which corresponds to the quadrupolar plasmon resonance condition (in the electrostatic approximation). To get around this divergence problem of the solutions, one could try to use a different solver in COMSOL and choose higher iterations, but it is very time-consuming. Therefore in this thesis, we will ignore parts of the spectra which the quadrupolar plasmon resonance occurs and leave the investigation of this region of the spectra for future studies.

\subsubsection{Single Spheroidal Particle}

In experimental nanophotonics, Mie theory cannot be used to provide solutions as the general geometries may involve elongated/pointly particles [83, 84, 85] (this is not as simple as spherical geometry which Mie theory can solve). In those non-spherical geometries such as nanorods and/or spheroids, new complicated issues may arise because of the lower symmetry of the problem. For example, stronger resonances, large field enhancements and gradients are typically exhibited at regions close to the tips of the particles. Therefore, our next to-do list is to run COMSOL simulations for elongated spheroidal particles, so the described situation above can be assessed. In doing so, we need to change the geometry of the particle from sphere to spheroid (or equivalently ellipsoid with $a=b$ and $c=3 a$ ), which is very straight forward. We are going to run simulations for two different parameters of the spheroid. First, we would like to set $a, b$ and $c$ such that the volume of the spheroid is equivalent to that of the $30 \mathrm{~nm}$ sphere, which leads to $a=a_{\text {sphere }} \times(1 / 3)^{1 / 3}=20.8 \mathrm{~nm}$. We also set $a=14.5 \mathrm{~nm}$ as another choice, so we can alter the mesh grid to reduce a bit of the simulation time, and this will be ideal when we seek the solutions for the core-shell system.

The results of the two spheroids (from COMSOL) will then be compared to the semi-analytic solution, so-called T-matrix whose simulations (in MatLab) can be found with the SMARTIES codes [86]. Since COMSOL simulation is timeconsuming, we shall consider only the silver particle for now. The results in Fig. 5.3 shows the comparison between the results from COMSOL versus those from SMARTIES (T-matrix) for two different-size silver spheroids (both with the aspect ratio of $h=c / a=3$ ). In both cases, both COMSOL and SMARTIES results are again in good agreement with the relative errors in the discrepancies $<\sim 1 \%$. This representation demonstrates that COMSOL is capable of simulating light scattering problems by particles of any shapes (as users can define/construct the geometry of the particle of their own choices). This is the demonstration of how to use COMSOL 

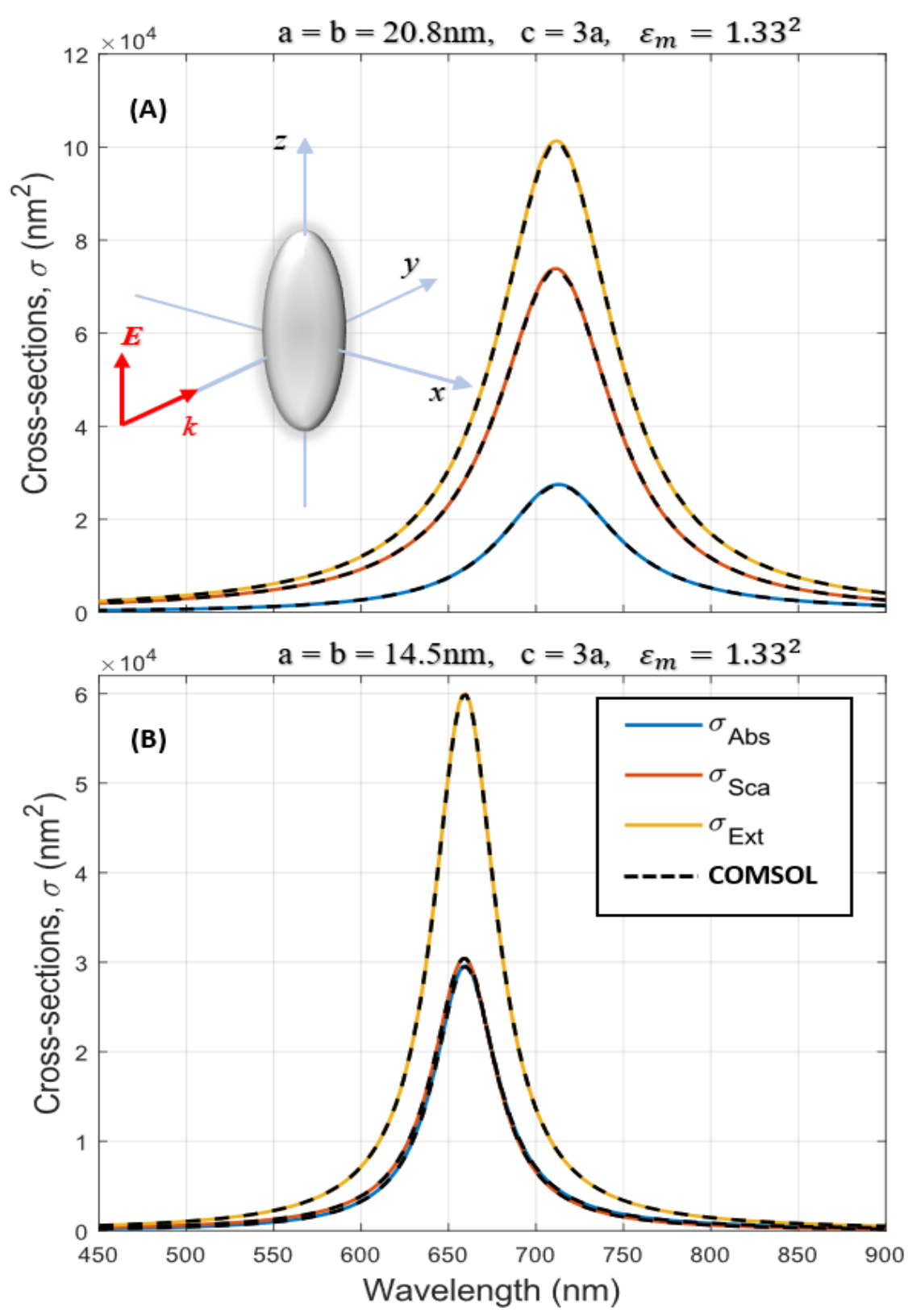

Figure 5.3: Comparison between FEM (COMSOL simulations) and T-matrix results for a silver spheroid embedded in water $\varepsilon_{\mathrm{m}}=1.33^{2}$ with (A) $a=b=20.8 \mathrm{~nm}$ and (B) $a=b=14.5 \mathrm{~nm}$, both cases the aspect ratio is chosen to be $h=3$. The results show the wavelength dependence of the absorption, scattering, and extinction cross-sections from T-matrix (colour lines) and COMSOL (dash lines). 
simulation to seek the light scattering solutions by non-spherical coated particles. Before this can be achieved, we need to know how the dielectric tensor for the coated layer can be implemented in COMSOL to reproduce the results for a spherical coreshell system (both, isotropic and anisotropic responses in the shell). Then, we can use that implementation as a tool to extend our study to a non-spherical coated particle, e.g. spheroids.

The other things to notice which were done differently for the spherical gold and silver particles (Figs. 5.1 and 5.2) and the spheroidal silver particles (Fig. 5.3) is the direction of the incident field. It is worth pointing out that for spherical particles, any plane wave incident field will give the same results as $\mathbf{E}_{\text {Inc }}$ defined in Eq. 5.2, which means it is independent of the polarisation and the field directions. This is due to the symmetry of the spherical geometry. However, this may not be the same case for spheroidal particles. In figure 5.3, we only show the results for $\mathbf{E}_{\text {Inc }}(\mathbf{r})=\mathrm{E}_{0} \exp \left(-i k_{\mathrm{m}} y\right) \hat{\mathbf{e}}_{z}$ which excites and induces the resonances shown in the figures and we may call them the long resonance in the case of spheroids. We can use SMARTIES to demonstrate (not shown here) that if the incident field was to be defined as $\mathbf{E}_{\mathrm{Inc}}(\mathbf{r})=\mathrm{E}_{0} \exp \left(-i k_{\mathrm{m}} z\right) \hat{\mathbf{e}}_{x}$ or $\mathrm{E}_{0} \exp \left(-i k_{\mathrm{m}} z\right) \hat{\mathbf{e}}_{y}$ then the induced resonances will be located on the left of the long resonances and they shall be called the short resonances. In this study of spheroidal particles, we will focus only on the long resonance for the time being, which will be presented throughout this chapter.

\subsection{Scattering by a Core-Shell System}

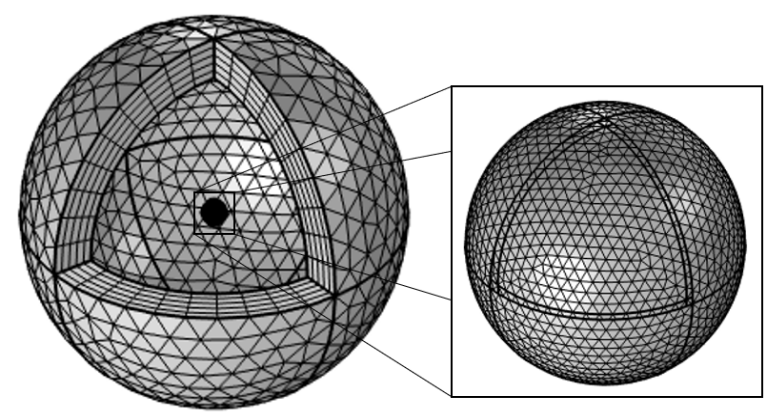

Figure 5.4: Geometry of the problem and grid mesh (PML on the left and the coated nanoparticle zoomed-in on the right) in COMSOL. 


\subsubsection{Spherical: Isotropic Core-Shell}

As we have shown that COMSOL can simulate particles of non-spherical shapes, we can now consider a coated layer consisting of dye-molecules surrounding the nanoparticle. To illustrate the range of capability of the COMSOL simulation, we will begin by reproducing the results for the light scattering problem by a spherical core-shell system and compare them to Mie theory.

To seek solutions for a coated spherical nanoparticle in COMSOL, we need to build a shell domain around the sphere and define its dielectric material. If the shell is isotropic, it will be simple. However, if it is an anisotropic layer, we need to define a tensor in the Cartesian basis to represent its dielectric functions. We shall first begin with the former case when the shell is isotropic. When we introduce an isotropic shell into our simulation, a new domain and surface must be defined. The absorption and the scattering of the core-shell system can then be calculated as:

$$
\begin{aligned}
\sigma_{\mathrm{Abs}} & =\frac{1}{S_{0}}\left[\iiint_{\mathrm{NP}} Q_{h} d V_{\mathrm{NP}}+\iiint_{\mathrm{Sh}} Q_{h} d V_{\mathrm{Sh}}\right], \\
\sigma_{\mathrm{Sca}} & =\frac{1}{S_{0}} \iint_{\mathrm{Sh}} \Re\left\{\mathbf{S}_{\mathrm{Sca}} \cdot \hat{\mathbf{n}}\right\} d S_{\mathrm{Sh}} .
\end{aligned}
$$

Furthermore, since the shell is considered to be isotropic, then the dielectric function for the shell can be easily defined as in the expression given in Eq. 4.12, then implement into the Material section (in COMSOL) for the shell domain.

The results from the COMSOL simulation and the comparison with Mie theory are shown in Fig. 5.5. We presented both the total absorption cross-section for the whole system (the core and the shell) in Fig. 5.5A, and the differential absorption cross-section, in Fig. 5.5B. Both results agree very well with Mie theory. This indicates that the core-shell system can be simulated within the COMSOL program for an isotropic shell.

\subsubsection{Spherical: Isotropic Core-Anisotropic Shell}

The Cartesian basis is preferred in COMSOL, therefore to implement the anisotropic effect (dielectric tensor) into the material for the shell domain, we have to define a new dielectric tensor in the Cartesian basis, instead of the spherical ones. Earlier, the dielectric tensor that was used in Mie theory was in the spherical basis, which 


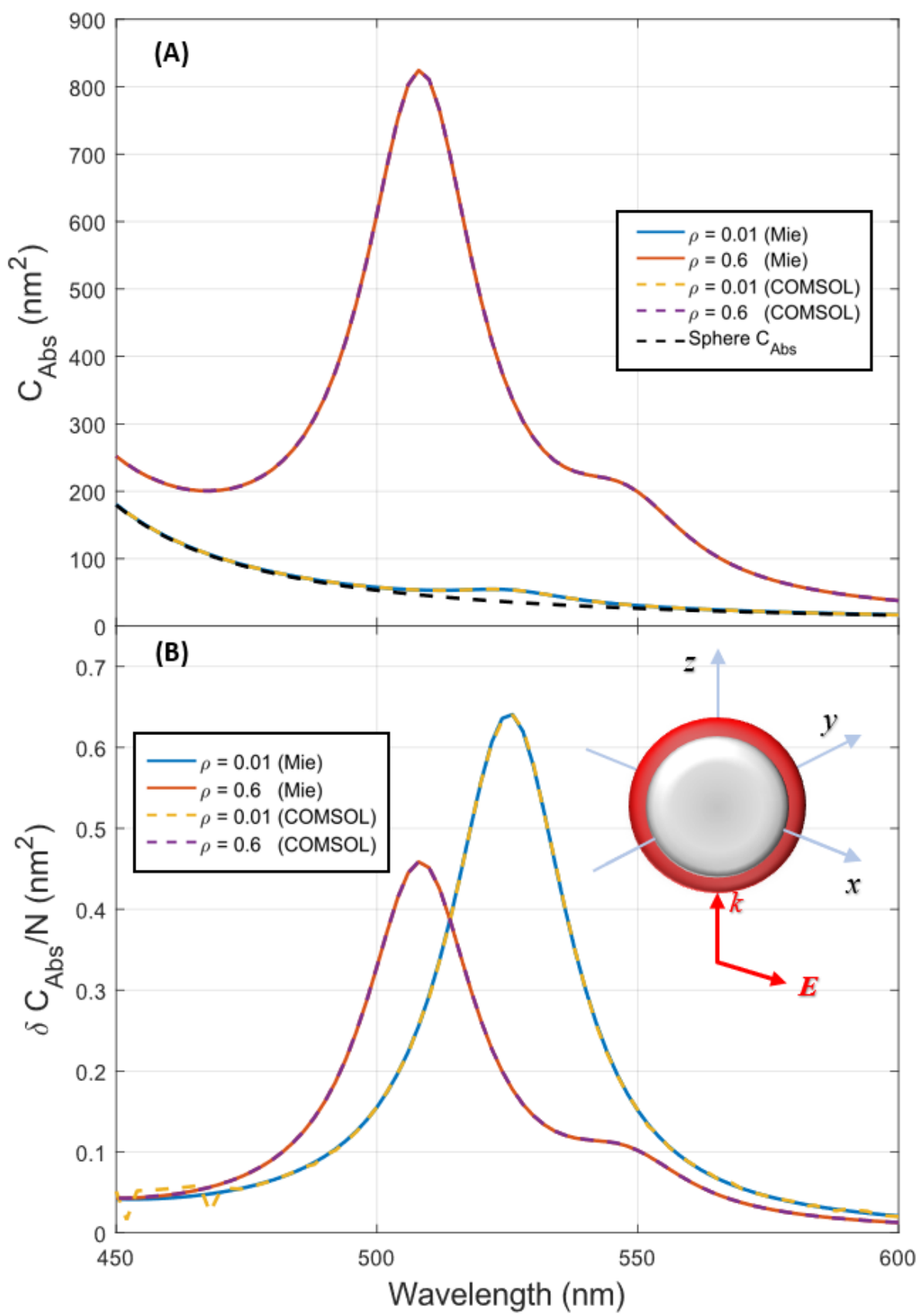

Figure 5.5: Comparison between FEM (COMSOL simulations) and Mie theory results for a silver sphere coated with an isotropic layer of dye-molecules embedded in water $\varepsilon_{\mathrm{m}}=1.33^{2}$. The results show the wavelength dependence of the total absorption crosssection (A) and the differential absorption cross-section (normalised by the number of dye-molecules) (B), from Mie theory (colour lines) and COMSOL (dash lines). The black dash line in (A) is absorption cross-section of the sphere only. 

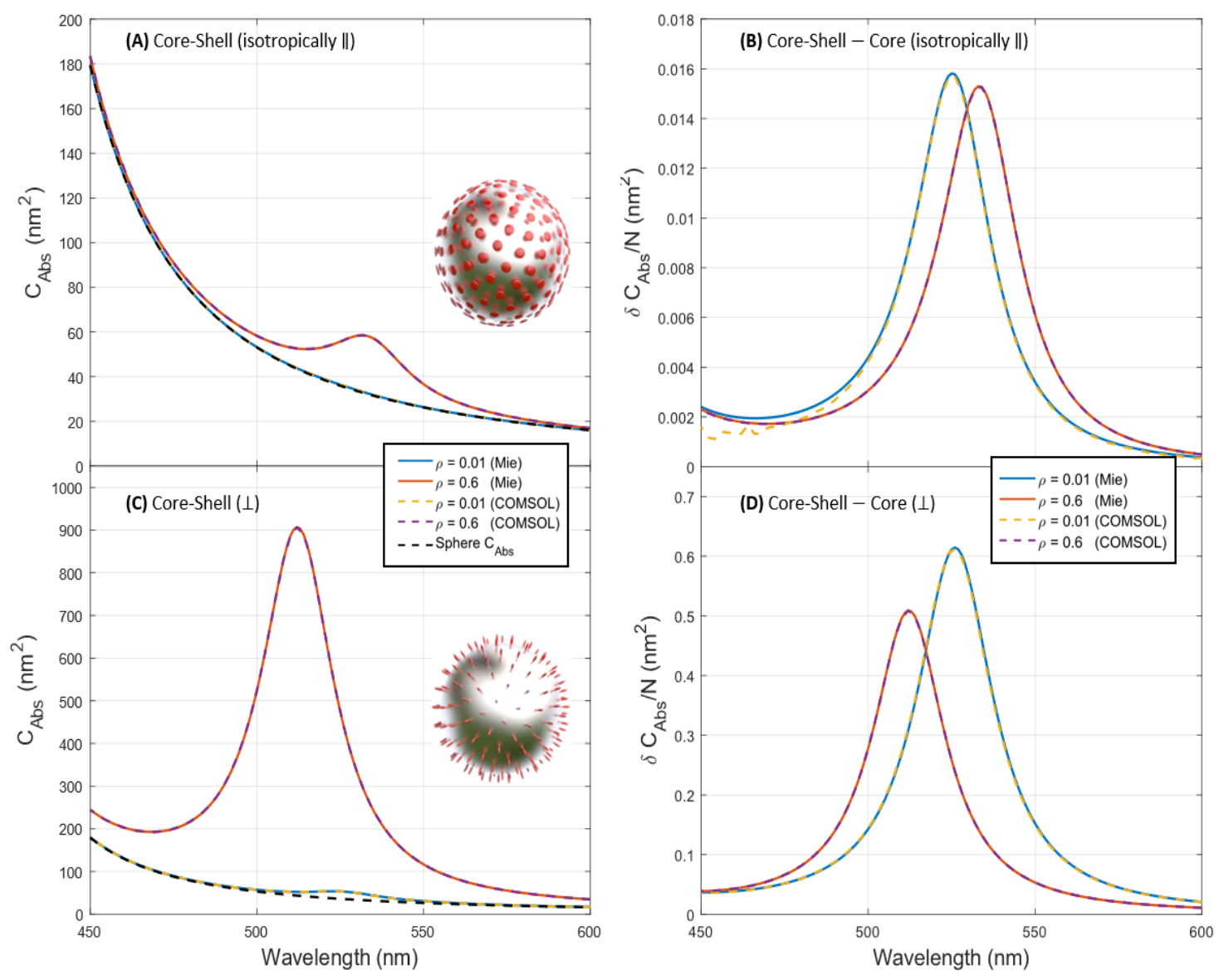

Figure 5.6: Comparison between FEM (COMSOL simulations) and Mie theory results for a silver sphere coated with an anisotropic layer of dye-molecules embedded in water $\varepsilon_{\mathrm{m}}=1.33^{2}$. The results show the wavelength dependence of the total absorption crosssection (A and $\mathrm{C}$ ) and the differential absorption cross-section (normalised by the number of dye-molecules) (B and D) for (isotropically in-plane molecules and radially pointed molecules, respectively), from Mie theory (colour lines) and COMSOL (dash lines). The black dash line in (A) is absorption cross-section of the sphere only. 
was defined as:

$$
\underline{\varepsilon}=\left(\begin{array}{ccc}
\varepsilon_{\mathrm{n}} & 0 & 0 \\
0 & \varepsilon_{\mathrm{t}} & 0 \\
0 & 0 & \varepsilon_{\mathrm{t}}
\end{array}\right)
$$

This dielectric tensor can be transformed into the Cartesian basis by using the transformation:

$$
\underline{\varepsilon}_{\text {Cart }}=\mathcal{S}_{\text {Sphere }}^{-1} \cdot \underline{\varepsilon} \cdot \mathcal{S}_{\text {Sphere }}
$$

where $\mathcal{S}_{\text {Sphere }}$ is the transformation matrix which can be used to transform Cartesian to Spherical coordinates. $\mathcal{S}_{\text {Sphere }}$ and its inverse $\mathcal{S}_{\text {Sphere }}^{-1}$ are given as:

$$
\begin{gathered}
\mathcal{S}_{\text {Sphere }}=\left[\begin{array}{ccc}
\sin \theta \cos \phi & \sin \theta \sin \phi & \cos \theta \\
\cos \theta \cos \phi & \cos \theta \sin \phi & -\sin \theta \\
-\sin \phi & \cos \phi & 0
\end{array}\right], \\
\mathcal{S}_{\text {Sphere }}^{-1}=\left[\begin{array}{ccc}
\sin \theta \cos \phi & \cos \theta \cos \phi & -\sin \phi \\
\sin \theta \sin \phi & \cos \theta \sin \phi & \cos \phi \\
\cos \theta & -\sin \theta & 0
\end{array}\right] .
\end{gathered}
$$

The expression in Eq. 5.10 can be found as follows, starting with $\mathbf{D}=\underline{\varepsilon} \mathbf{E}$ :

$$
\begin{aligned}
\mathbf{D}_{\text {Cart }}=\underline{\varepsilon}_{\text {Cart }} \mathbf{E}_{\text {Cart }} & =\mathcal{S}_{\text {Sphere }}^{-1} \mathbf{D}_{\text {Sphere }} \\
& =\mathcal{S}_{\text {Sphere }}^{-1} \underline{\varepsilon} \mathbf{E}_{\text {Sphere }} \\
& =\mathcal{S}_{\text {Sphere }}^{-1} \underline{\varepsilon} \mathcal{S}_{\text {Sphere }} \mathbf{E}_{\text {Cart }} .
\end{aligned}
$$

The angles $\phi$ and $\theta$ can be calculated from the points on the surface of the shell (according to the choice of grid mesh). For any point on the spherical shell represented by $(x, y, z)$ in the Cartesian coordinates, we can extract $\phi$ and $\theta$ as:

$$
\phi=\arctan 2\left(\frac{y}{x}\right), \quad \theta=\arctan 2\left(\frac{z}{\sqrt{x^{2}+y^{2}}}\right) .
$$

By implementing the above formalism, which describes the material properties (via dielectric tensor) into COMSOL, we can simply rerun the simulation as done for an isotropic core-shell case and produce the results illustrated in Fig. 5.6. As we should expect, the results show that the COMSOL simulations have provided an indistinguishable comparison to Mie theory for absorption and differential absorption cross-sections for both cases of the orientations of the dye-molecules (isotropically in-plane and radial orientations). 


\subsubsection{Spheroidal: Isotropic Core-Anisotropic Shell}

The results above indicate that the anisotropic effect in the shell can indeed be accounted for in COMSOL, and they yield the same results as one should expect from Mie theory. We now may use this as a formalism to do the same thing for a coated spheroidal particle. This is the first step toward investigating the extension of the light scattering problem by a spherical particle (coated) to a non-spherical coated particle. Similar to the spherical coated particle case, we need to find the transformation matrix $\mathcal{S}_{\text {Spheroid }}$ and its inverse $\mathcal{S}_{\text {Spheroid }}^{-1}$, which link the Spheroidal coordinates to Cartesian coordinates, and vice-versa. To do so, we will start with a point $(x, y, z)$ in Cartesian coordinates and seek the transformation matrix, which transforms it to a point in Spheroidal coordinates $(\xi, \zeta, \eta)$. We can begin with:

$$
\begin{aligned}
& x=a \sin \alpha \cos \beta, \\
& y=a \sin \alpha \sin \beta, \\
& z=c \cos \alpha .
\end{aligned}
$$

$a<c$, Prolate spheroid, $a>c$, Oblate spheroid.

The angles $\alpha$ and $\beta$ can be found (similar to the spherical case, $\theta$ and $\phi$, respectively) as:

$$
\alpha=\arctan 2\left(\frac{z / c}{\sqrt{x^{2} / a^{2}+y^{2} / a^{2}}}\right), \quad \beta=\arctan 2\left(\frac{y}{x}\right)
$$

The vector $\underline{\xi}$ is given as:

$$
\underline{\xi}=\frac{\sin \alpha \cos \beta}{a} \hat{\mathbf{e}}_{x}+\frac{\sin \alpha \sin \beta}{a} \hat{\mathbf{e}}_{y}+\frac{\cos \alpha}{c} \hat{\mathbf{e}}_{z}
$$

and

$$
\begin{aligned}
\hat{\mathbf{e}}_{\xi} & =\|\underline{\xi}\|^{-1} \underline{\xi} \\
& =\left[\frac{x^{2}}{a^{4}}+\frac{y^{2}}{a^{4}}+\frac{z^{2}}{c^{4}}\right]^{-1 / 2}\left[\frac{x}{a^{2}} \hat{\mathbf{e}}_{x}+\frac{y}{a^{2}} \hat{\mathbf{e}}_{y}+\frac{z}{c^{2}} \hat{\mathbf{e}}_{z}\right] .
\end{aligned}
$$

The other two orthogonal vectors to $\underline{\xi}$ are $\underline{\zeta}$ and $\underline{\eta}$ and they can be found as:

$$
\begin{aligned}
& \underline{\zeta}=a \cos \alpha \cos \beta \hat{\mathbf{e}}_{x}+a \cos \alpha \sin \beta \hat{\mathbf{e}}_{y}-c \sin \alpha \hat{\mathbf{e}}_{z} \\
& \underline{\eta}=-a \sin \alpha \sin \beta \hat{\mathbf{e}}_{x}+a \sin \alpha \cos \beta \hat{\mathbf{e}}_{y} .
\end{aligned}
$$

Therefore, the Spheroidal and Cartesian coordinates are related by equation below via the transformation matrix, $\mathcal{S}_{\text {Spheroid }}$ : 


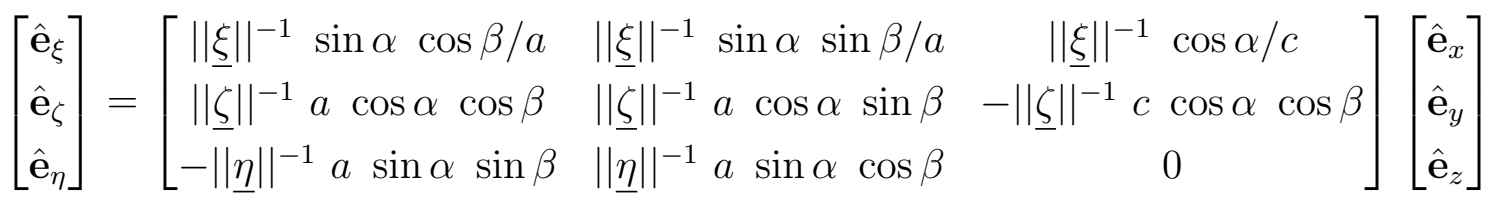

$$
\begin{aligned}
& =\mathcal{S}_{\text {Spheroid }}\left[\begin{array}{c}
\hat{\mathbf{e}}_{x} \\
\hat{\mathbf{e}}_{y} \\
\hat{\mathbf{e}}_{z}
\end{array}\right]
\end{aligned}
$$

and the inverse transformation matrix can be found as,

$$
\begin{gathered}
\mathcal{S}_{\text {Spheroid }}^{-1}=\mathcal{F}\left[\begin{array}{lll}
\underline{\nu}_{1} & \underline{\nu}_{2} & \underline{\nu}_{3}
\end{array}\right], \\
\text { where } \\
\mathcal{F}=\frac{1}{a^{2}\left(c^{2} \sin ^{2} \alpha+a^{2} \cos ^{2} \alpha\right)}, \\
\underline{\nu}_{1}=\|\underline{\xi}\|\left[\begin{array}{c}
a^{3} c^{2} \sin \alpha \cos \beta \\
a^{3} c^{2} \sin \alpha \sin \beta \\
a^{4} c \cos \alpha
\end{array}\right], \quad \underline{\nu}_{2}=\|\underline{\zeta}\|\left[\begin{array}{c}
a^{3} \cos \alpha \cos \beta \\
a^{3} \cos \alpha \sin \beta \\
-a^{2} c \sin \alpha
\end{array}\right], \\
\underline{\nu}_{3}=\|\underline{\eta}\|\left[\begin{array}{c}
-\left(a^{3} \cos \alpha \cot \alpha+a c^{2} \sin \alpha\right) \sin \beta \\
\left(a^{3} \cos \alpha \cot \alpha+a c^{2} \sin \alpha\right) \cos \beta \\
0
\end{array}\right] .
\end{gathered}
$$

The dielectric tensor for anisotropic spheroidal shell in the Cartesian basis can then be calculated as:

$$
\underline{\varepsilon}_{\text {Cart }}=\mathcal{S}_{\text {Spheroid }}^{-1} \underline{\varepsilon}_{\text {Shell }} \mathcal{S}_{\text {Spheroid }} .
$$

We shall now implement these into COMSOL for the properties of the anisotropic spheroidal shell. The results are shown in Fig. 5.7. The results shown on the left (Fig. 5.7A and C) correspond to the differential absorption cross-sections when the incident field is chosen to be $\mathbf{E}_{\text {Inc }}(\mathbf{r})=\mathrm{E}_{0} \exp \left(-i k_{\mathrm{m}} x\right) \hat{\mathbf{e}}_{z}$. And the right of Fig. 5.7 (B and $\mathrm{D})$, it corresponds to the incident field being $\mathbf{E}_{\text {Inc }}(\mathbf{r})=\mathrm{E}_{0} \exp \left(-i k_{\mathrm{m}} z\right) \hat{\mathbf{e}}_{x}$. This figure indicates that different polarization of the incident electric field can lead to different results in the differential absorption cross-sections for the anisotropic coated spheroidal particle. We observe different amplitudes in the spectra, especially in the case of smarties-like dipoles. In this case, however, the shift in the 

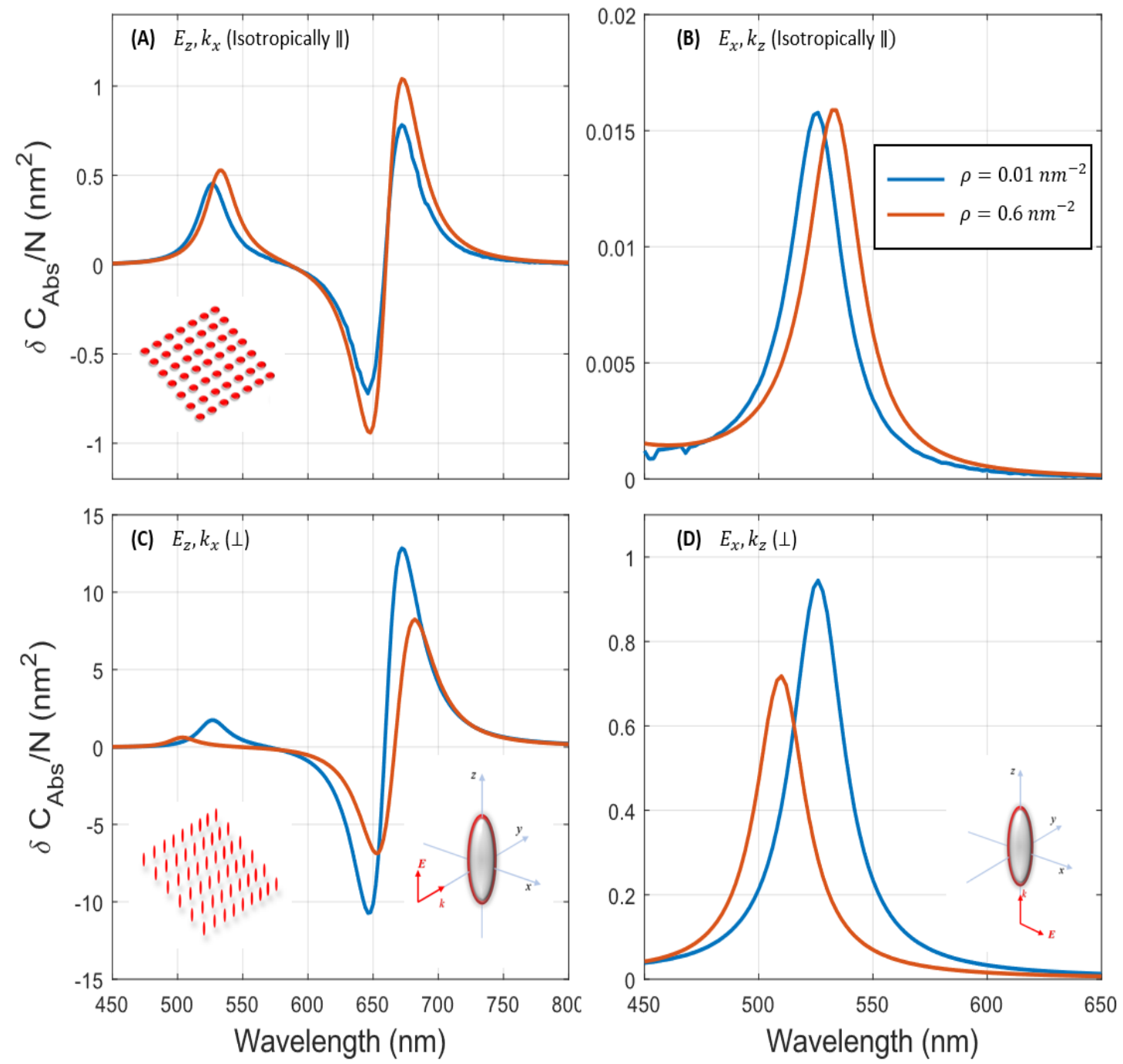

Figure 5.7: Calculations from FEM (COMSOL simulations) for a silver prolate spheroid ( $a=14.5 \mathrm{~nm}$, and $h=3$ ) coated with an anisotropic layer of dye-molecules (thickness, $l_{s}=1 \mathrm{~nm}$ ) embedded in water $\varepsilon_{\mathrm{m}}=1.33^{2}$. The results show the wavelength dependence of the normalised differential absorption cross-sections for smarties-like dipoles (top row) and radially pointed dipoles (bottom row). Different polarisation in the electric incident field are shown in $(\mathrm{A}$ and $\mathrm{C})$ for $\mathrm{E}_{\mathrm{Inc}}(\mathbf{r})=\mathrm{E}_{0} \exp \left(-i k_{\mathrm{m}} x\right) \hat{\mathbf{e}}_{z}$, and (B and D) for $\mathbf{E}_{\text {Inc }}(\mathbf{r})=\mathrm{E}_{0} \exp \left(-i k_{\mathrm{m}} z\right) \hat{\mathbf{e}}_{x}$. 


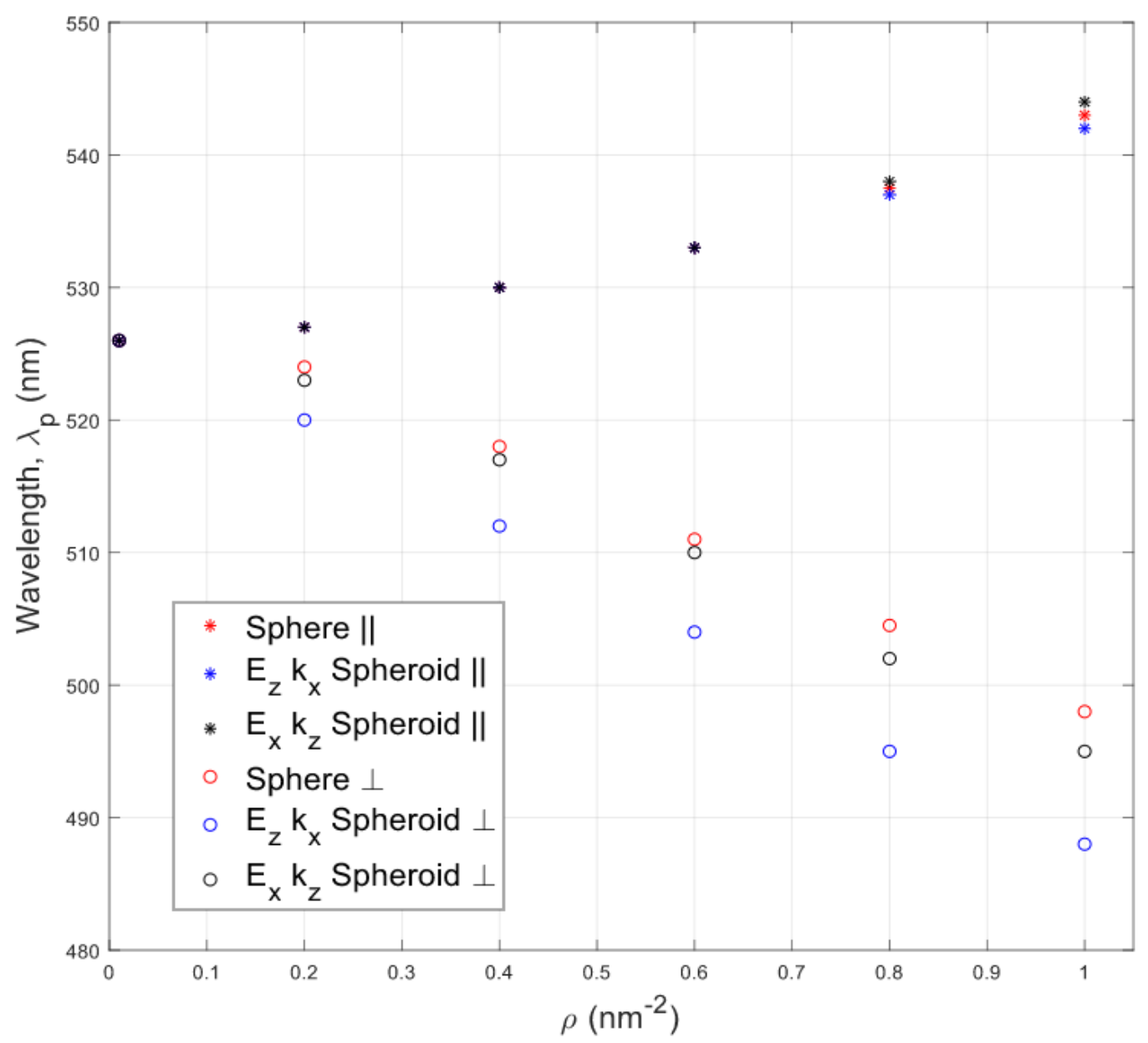

Figure 5.8: The wavelength at the dye-resonance $\lambda_{p}$ as a function of the dye coverage showing in $[*]$ for smarties-like dipoles $(\|)$ and $[\circ]$ for radially pointed dipoles $(\perp)$ for three different cases, spherical (in red), spheroidal with $\mathbf{E}_{\mathrm{Inc}}(\mathbf{r})=\mathrm{E}_{0} \exp \left(-i k_{\mathrm{m}} x\right) \hat{\mathbf{e}}_{z}$ (in blue), and spheroidal with $\mathbf{E}_{\text {Inc }}(\mathbf{r})=\mathrm{E}_{0} \exp \left(-i k_{\mathrm{m}} z\right) \hat{\mathbf{e}}_{x}$ (in black). 
dye-resonance appears to remain unchanged (for both $\rho=0.01$ and $0.6 \mathrm{~nm}^{-2}$ ). But in the case of radially pointed dipoles, we notice both the differences in the amplitudes and the shifts in the dye-resonance.

In figures 5.7A and $\mathrm{C}$, we have also included the plasmon resonance region ( $\lambda$ is between $600-750 \mathrm{~nm})^{4}$. The spectra show the differential-like shape, which suggests the plasmon resonance has been red-shifted (for both orientations, in this case) due to the effect of the dye-molecules adsorbed on the silver particle. The effects which may cause the shift in the plasmon resonance will be discussed in more details in the next chapter. Another important fact that we can look into in more details is what happens when the dye's resonance and the plasmon resonance are overlapping (i.e., strong resonance coupling region).

Figure 5.8 illustrates how the dye-resonance shifted according to the dye orientations and the polarisations of the incident field in the spheroidal case. For the smartie-like dipoles adsorbed onto either spherical or spheroidal silver particle, the shift in the dye's resonance remains the same for up to the dye-coverage of $\rho=0.6 \mathrm{~nm}^{-2}$, and starts to show little discrepancies for $\rho>0.8 \mathrm{~nm}^{-2}$, as shown in Fig. 5.8 (displayed as *). The discrepancies in the dye-resonance shift for the $\perp$ case begin from $\rho>0.01 \mathrm{~nm}^{-2}$. We observe that the more blue-shifts for spheroidal cases compare to the spherical case. The polarisation of the incident field $\mathbf{E}_{\text {Inc }}(\mathbf{r})=\mathrm{E}_{0} \exp \left(-i k_{\mathrm{m}} x\right) \hat{\mathbf{e}}_{z}$ leads to more pronounced blue-shifts in the dye's resonance than $\mathbf{E}_{\mathrm{Inc}}(\mathbf{r})=\mathrm{E}_{0} \exp \left(-i k_{\mathrm{m}} z\right) \hat{\mathbf{e}}_{x}$. This is an interesting result, which provides evidence that the polarisation of the incident field can affect the shift in the dye's resonance depend on the dye-coverage, for non-spherical coated nanoparticle.

\subsection{Conclusion}

This chapter demonstrates how FEM calculation can be practically implemented in COMSOL to investigate the light scattering problem by spherical and spheroidal nanoparticles coated with an anisotropic layer of dye-molecules. This work has provided a tool to study light scattering problem by objects with non-spherical shapes, which leads to interesting results of how the optical properties of the system are related to the polarisation of the incident electric field. Another possible study with the same tool would be to investigate how the location of the dye molecules

\footnotetext{
${ }^{4}$ For $E_{x}, k_{z}$ incident field, the plasmon resonance occurs when $\lambda<375 \mathrm{~nm}$, and we ignore this region as discussed earlier. So, we are only showing results at the dye's resonance region only.
} 
can affect the overall results. This aspect will be briefly considered in Chapter 6 . 


\section{The Effects of Anisotropic Molecules on the Nanoparticles' Plasmon Resonance}

In previous chapters, we have focused our interest on how the electronic resonances of dye molecules change due to the effects from their adsorbed orientations, surface coverage, local-field corrections (interaction between their neighbouring molecules), and from the metallic nanoparticle. In contrast to that, we will now study how these effects will affect the localised surface plasmon resonance (LSPR) of the nanoparticles. Previous studies have been shown that the peak position $\left(\lambda_{\mathrm{PR}}\right)$ of the LSPR for metallic nanoparticles is highly dependent upon the refractive index of the surrounding medium [87]. It is also well-documented and explained in Ref. [25] how resonant adsorbates (specifically Rhodamine 6G) influence the LSPR of Ag nanoparticles. However, how the orientation of the resonant adsorbates may affect the plasmon resonance has not been discussed. Therefore, we will investigate and study the dyeorientation effects on the plasmon resonance.

In most of the theoretical considerations for the core/shell system, the dyemolecules are commonly assumed to surround the nanoparticle with an even distribution across the whole nanoparticle's surface. From an experimental point of view, this should not be a problem for a high surface-coverage of the molecules or colloids in dye's solution. However, for some cases (i.e., low surface coverage, for example), the dye-molecules may not be evenly distributed on the nanoparticle's surface. In that situation, the locations of the adsorbed molecules will become significantly important, especially when they are adsorbed onto special types of nanoparticles whose surfaces have more than one distinct physical properties, known as Janus particles $[88,89]$. Therefore in this chapter, we will also investigate how the dye-location may affect the plasmon resonance. 


\subsection{Refractive Index Dependence}

Let us first present results from previous studies on how the peak of LSPR is shifted depending on the embedding medium of the nanoparticles (i.e. the refractive index or the dielectric constant of the surrounding medium). According to Ref. [87], we can find the relationship between the peak wavelength of the LSPR and the dielectric constant for the medium for small NPs in the electrostatic approximation [27]. For the main dipolar LSPR, the resonance occurs when:

$$
\Re\left(\varepsilon_{\mathrm{Ag}}\right) \rightarrow-2 \varepsilon_{\mathrm{m}} .
$$

Then, we can use the analytical frequency-dependent form for $\varepsilon_{\mathrm{Ag}}$ from the Drude model of the electronic structure of metals:

$$
\Re\left(\varepsilon_{\mathrm{Ag}}\right)=1-\frac{\omega_{\mathrm{p}}^{2}}{\omega^{2}-\gamma^{2}},
$$

where $\omega_{\mathrm{p}}$ is the plasmon frequency and $\gamma$ is the damping frequency of the bulk metal. For the near-infrared, visible, and ultra-violet frequencies, $\gamma \ll \omega_{\mathrm{p}}$ so we can simplify the above equation to:

$$
\Re\left(\varepsilon_{\mathrm{Ag}}\right)=1-\frac{\omega_{\mathrm{p}}^{2}}{\omega^{2}},
$$

At resonance, we have:

$$
\lambda_{\mathrm{PR}}=\lambda_{\mathrm{p}} \sqrt{2 \varepsilon_{\mathrm{m}}+1}
$$

where $\lambda_{\mathrm{p}}$ is the plasmon wavelength. According to Eq. 6.4, we can conclude that if the dielectric constant of the embedding medium is increased (decreased), then $\lambda_{\mathrm{PR}}$ will increase (decrease). We can use Mie theory to provide evidence for this, as shown in Fig. 6.1. For a silver sphere of radius $14 \mathrm{~nm}$ embedded in water $\varepsilon_{\mathrm{m}}=1.33^{2}$, the main dipolar LSPR is at $\lambda_{\mathrm{PR}}=397 \mathrm{~nm}$. If we decrease the value of $\varepsilon_{\mathrm{m}}$, the absorption cross-section spectrum is blue-shifted (as shown for $\varepsilon_{\mathrm{m}}=1$, and 1.5). And the red-shifts in the LSPR are observed if we set $\varepsilon_{\mathrm{m}}$ to be higher than $1.33^{2}$ (i.e 2 and 2.5). [Note that from this section 6.1 to 6.2 , the results were computed by Mie theory for the demonstrations]. 


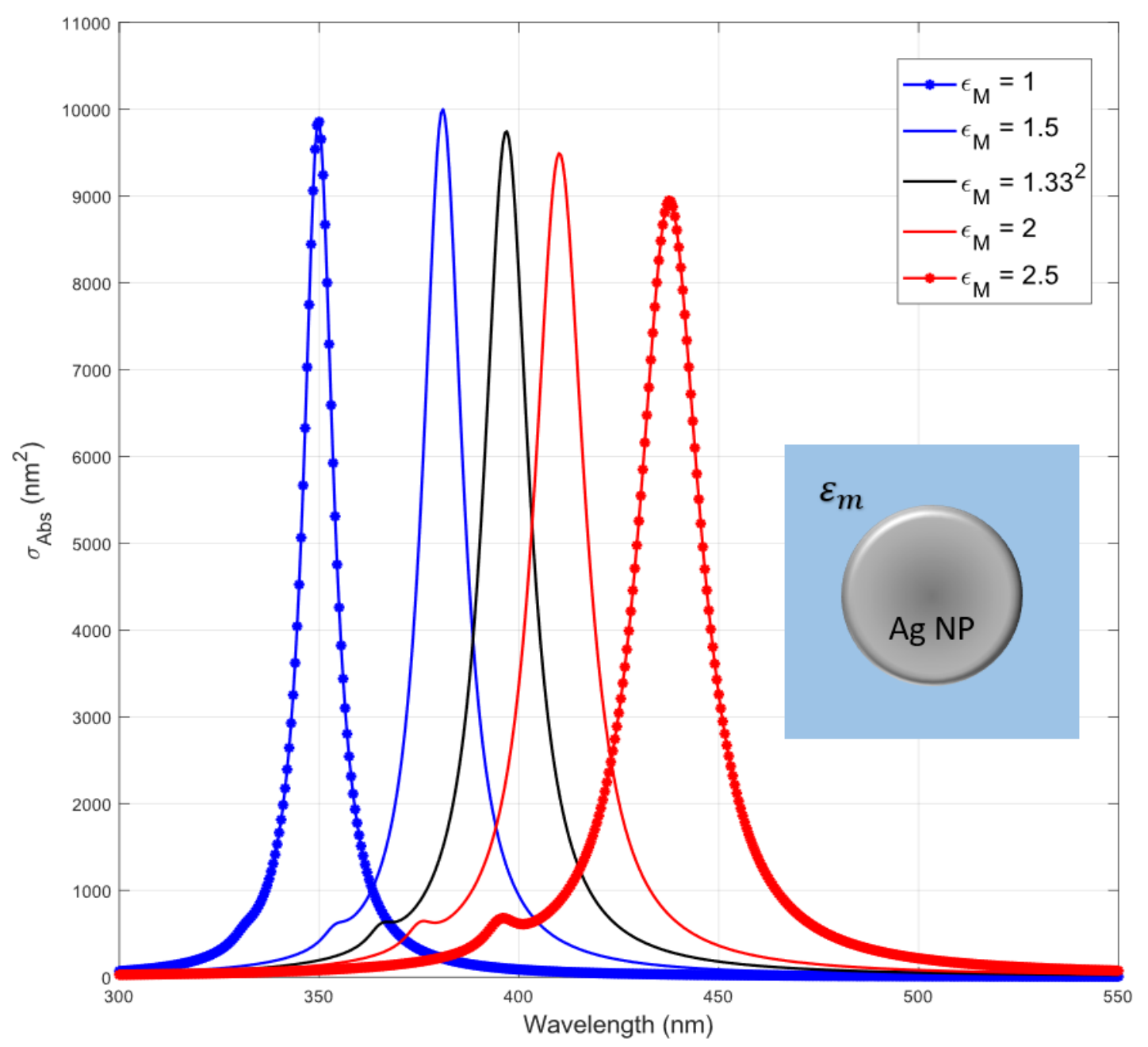

Figure 6.1: Absorption cross-section spectra for a silver sphere of radius $a=14 \mathrm{~nm}$ embedded in a medium of $\varepsilon_{\mathrm{m}}$. The spectrum for the dielectric constant of the medium $\varepsilon_{\mathrm{m}}=1.33^{2}$ is shown in black, for $\varepsilon_{\mathrm{m}}<1.33^{2}$ are shown in blue (as the LSPR is blueshifted), and for $\varepsilon_{\mathrm{m}}>1.33^{2}$ are shown in red (for red-shifted LSPR). 

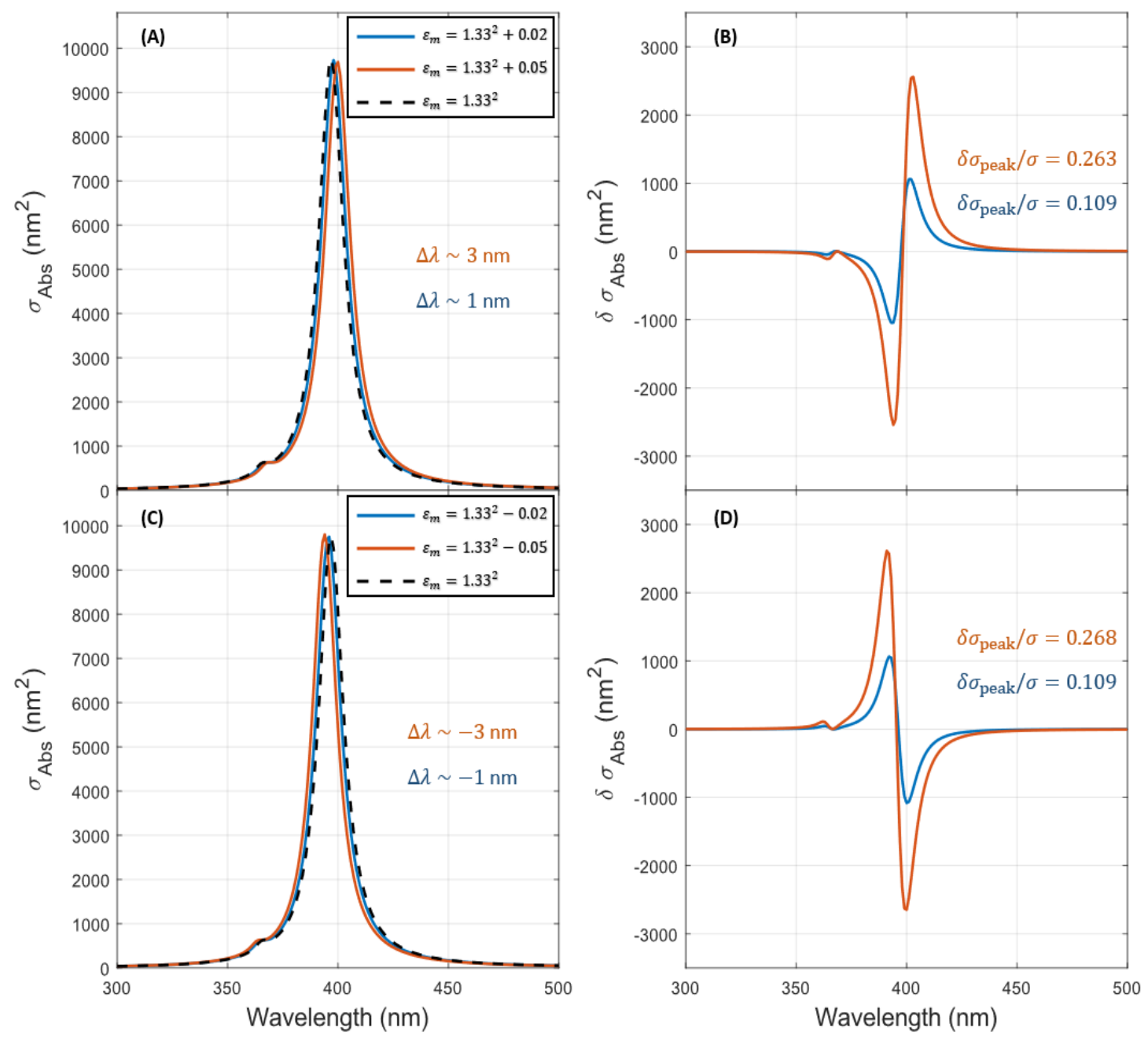

Figure 6.2: Absorption cross-section spectra for a silver sphere of radius $a=14 \mathrm{~nm}$ embedded in a medium of $\varepsilon_{\mathrm{m}}$. The absorption cross-section spectra for the particle embedded in an external medium of $\varepsilon_{\mathrm{m}}>1.33^{2}$ (shown in A) and $\varepsilon_{\mathrm{m}}<1.33^{2}$ (shown in $\mathbf{C})$. Their corresponding differential absorption cross-sections $\left(\sigma_{\mathrm{Abs}}-\sigma_{\mathrm{Abs}, 1.33^{2}}\right)$ are shown in (B) and (D), respectively. 
In figure 6.1, we can observe the shifts in the LSPR very clearly because the peak wavelengths (at which the resonance occurs) are distinctively separated. If the peak wavelengths are very close to one another (as shown in Fig. 6.2 A and C), the shifts may not be accurately concluded by just looking at the absorption cross-section spectra. Therefore, we need to look at the differential absorption crosssection $\left(\sigma_{\mathrm{Abs}}\right.$ of the spectra that we are interested in minus $\sigma_{\mathrm{Abs}}$ of the one we want to compare to). In this case, we treat $\varepsilon_{m}=1.33^{2}$ as the reference (the one we want to compare to). We can see the characteristic derivative-like spectral shapes by taking the differential absorption cross-section spectra as our observation (shown in Fig. 6.2 $\mathrm{B}$ and $\mathrm{D}$ ). In figure $6.2 \mathrm{~B}$, the negative values of the differential absorption crosssection are on the left and the positive values are on the right, which corresponds to the spectrum being shifted to the right, i.e. red-shifted. In contrast to that, Fig. 6.2D, shows a different (the opposite) differential-like shape compared to Fig. $6.2 \mathrm{~B}$, which indicates that the spectrum has been shifted to the left, i.e. blueshifted. The intensity of the differential absorption cross-section represents the magnitude of the shift in the LSPR. We can relate $\delta \sigma_{\mathrm{Abs}} / \sigma_{\mathrm{Abs}}$ to the shift in the wavelength as, $\Delta \lambda \sim 10 \times \delta \sigma_{\mathrm{Abs}} / \sigma_{\mathrm{Abs}}$. From figure $6.2 \mathrm{~B}$, we can conclude that for $\varepsilon_{\mathrm{m}}=1.33^{2}+0.05$ the LSPR has been blue-shifted $\sim 2.5$ times more compared to the case when $\varepsilon_{\mathrm{m}}=1.33^{2}+0.02$. Therefore, we will be able to decide whether the LSPR is either red-shifted or blue-shifted from these derivative-like shapes. If the spectra in Fig. 6.2 B (or D) are observed, we can conclude that the LSPR is red-shifted (or blue-shifted).

\subsection{Dye's Properties Dependence}

In this section, we will investigate how the LSPR of the silver nanoparticle will be affected when the nanoparticle is surrounded by dye-molecules. So far, we have shown the effect of the external dielectric medium on the LSPR for the silver nanoparticles. The results indicate the red-shift (blue-shift) in the absorption cross-section spectrum is due to the increase (or decrease) in the dielectric constant of the external medium. Therefore, for the dye-coated nanoparticle, the shifts in the plasmon resonance will depend on the dielectric function of the dye-molecule (shell) medium.

\subsubsection{Dye's Polarisability Dependence}

We first consider isotropic dye-molecules adsorbed onto a silver nanoparticle. The dielectric function for an isotropic shell of molecules was previously defined in Eq. 
4.12 as:

$$
\varepsilon_{\mathrm{eff}}(\lambda)=\varepsilon_{\mathrm{m}}+\frac{L_{\mathrm{m}}^{2} c_{\mathrm{d}} \alpha_{\mathrm{d}} / \varepsilon_{0}}{1-\left(L_{\mathrm{m}}^{2} / \varepsilon_{\mathrm{m}}\right) c_{\mathrm{d}} \alpha_{\mathrm{d}} /\left(3 \varepsilon_{0}\right)}
$$

Therefore, the shift in LSPR will then depend on the second term of this equation, or equivalently, the polarisability of the molecules. By assuming $\Im\left\{\alpha_{\mathrm{d}}\right\} \ll \Re\left\{\alpha_{\mathrm{d}}\right\}$, we have the following conditions:

- If $\Re\left\{\alpha_{\mathrm{d}}\left(\lambda_{\mathrm{PR}}\right)\right\}=0$, then there will not be any shift in the plasmon resonance at all.

- If $0<\Re\left\{\alpha_{\mathrm{d}}\left(\lambda_{\mathrm{PR}}\right)\right\}<3 \varepsilon_{0} \varepsilon_{\mathrm{m}} /\left(L_{\mathrm{m}}^{2} c_{\mathrm{d}}\right)$, the LSPR will be red-shifted.

- And if $\Re\left\{\alpha_{\mathrm{d}}\left(\lambda_{\mathrm{PR}}\right)\right\}<0$ or $\Re\left\{\alpha_{\mathrm{d}}\left(\lambda_{\mathrm{PR}}\right)\right\}>3 \varepsilon_{0} \varepsilon_{\mathrm{m}} /\left(L_{\mathrm{m}}^{2} c_{\mathrm{d}}\right)$, the blue-shift in the LSPR will be observed.

\subsubsection{Dye Molecules Adsorbed on the Ag Sphere}

We are now going to re-visit the case of Rhodamine 6G molecules (treated as isotropic) adsorbed onto a silver nanosphere. The polarisability of the Rhodamine $6 \mathrm{G}$ molecules is given in Eq. 3.77 as:

$$
\alpha_{\mathrm{d}}=\alpha_{\infty}-\frac{\alpha_{1} \lambda_{1}}{\mu_{1}}\left[1-\frac{1}{1-\frac{\lambda_{1}^{2}}{\lambda^{2}}-i \frac{\lambda_{1}^{2}}{\lambda \mu_{1}}}\right],
$$

where

$$
\begin{array}{lc}
\alpha_{\infty}=9.4 \times 10^{-39}[\mathrm{SI}], & \alpha_{1}=5.76 \times 10^{-38}[\mathrm{SI}], \\
\lambda_{1}=526 \mathrm{~nm}, & \mu_{1}=10000 \mathrm{~nm} .
\end{array}
$$

With these parameters and for low dye-coverage $\rho=0.01 \mathrm{~nm}^{-2}$, the second condition is satisfied, i.e. we should expect the red-shift in the LSPR shown in Fig. 6.3 A. Mathematically, the value of $\Re\left\{\alpha_{\mathrm{d}}\left(\lambda_{\mathrm{PR}}\right)\right\}$ literally depends on the parameters in Eq. 6.7. Therefore, altering one (or more) of these parameters will affect the shift in the LSPR. We demonstrate that in Fig. 6.3, where we change the value of $\alpha_{1}$, and the rest of the parameters remain unchanged. For the polarisability of Rhodamine 6G, we observe red-shift in the plasmon resonance as shown in Fig. 6.3 A. When $\alpha_{1}$ is changed to $8.00 \times 10^{-38}$ (in Fig. $6.3 \mathrm{~B}$ ), the shift in the main dipolar plasmon resonance disappeared, but we can still observe the red-shift in the quadrupolar resonance (at $\lambda \sim 368 \mathrm{~nm}$ ). And in figure $6.3 \mathrm{C}$, the value of $\alpha_{1}$ is further increased to $1.30 \times 10^{-37}$, the main resonance (as well as the quadrupolar resonance) is blueshifted. 


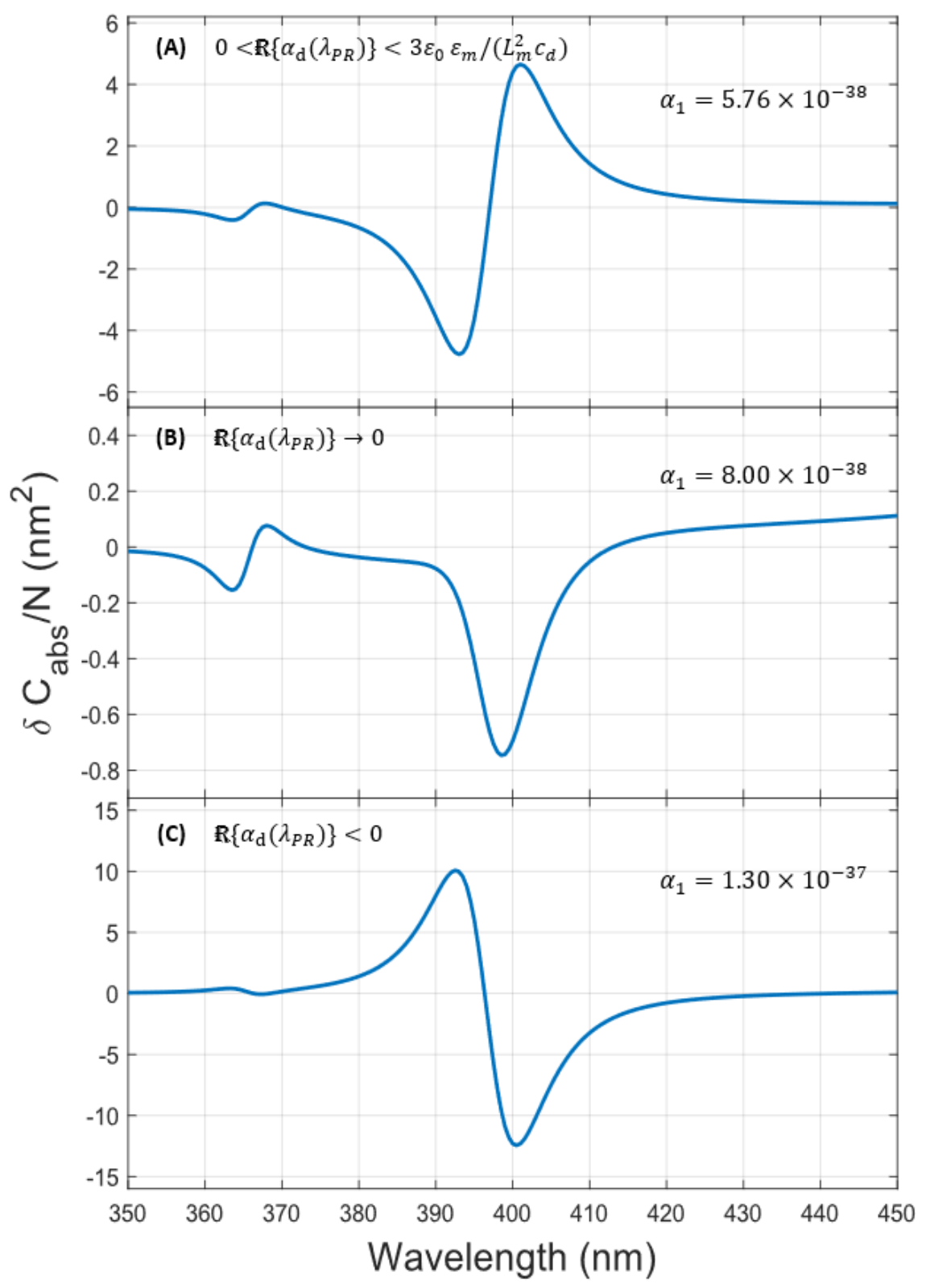

Figure 6.3: Normalised differential absorption cross-section showing different shifts in LSPR for different values of $\alpha_{1}$. For $\alpha_{1}=5.76 \times 10^{-38}$, a red-shift is observed and shown in (A). No shift in the main LSPR for $\alpha_{1}=8.00 \times 10^{-38}$, shown in (B). And in (C), a blue-shift is shown for $\alpha_{1}=1.30 \times 10^{-37}$. 
If we increase the dye-coverage to a value of $c_{\mathrm{d}}>L_{\mathrm{m}}^{2} \Re\left\{\alpha_{\mathrm{d}}\left(\lambda_{\mathrm{PR}}\right)\right\} /\left(3 \varepsilon_{0} \varepsilon_{\mathrm{m}}\right)$ (not shown here, but will be demonstrated in the next section), the only feature that will change in Fig. 6.3 would be the LSPR shift in Fig. 6.3 A. In that case, a blue-shift in the LSPR will be expected instead of the red-shift. However, the shape of the spectra observed in Fig. 6.3 B and $\mathrm{C}$ should remain the same.

\subsubsection{Dye's Orientation Dependence}

In this section, we will discuss the effect of anisotropic dye molecules (Rhodamine $6 \mathrm{G}$ ) on the plasmon resonance in details. We may begin with the anisotropic EMM for the dielectric functions of the anisotropic shell. Recall from Eqs. 4.24 and 4.26, we have:

$$
\begin{aligned}
& \varepsilon_{x y}=\varepsilon_{\mathrm{m}}+\frac{L_{\mathrm{m}}^{2} c_{\mathrm{d}} \alpha_{\mathrm{d}} /\left(2 \varepsilon_{0}\right)}{1-\frac{\alpha_{\mathrm{d}}}{8 \pi \varepsilon_{0}} \frac{L_{\mathrm{m}}^{2}}{\varepsilon_{\mathrm{m}}} \rho^{3 / 2} \xi_{0} / 2}, \\
& \frac{1}{\varepsilon_{z z}}=\frac{1}{\varepsilon_{\mathrm{m}}}-\frac{\left(L_{\mathrm{m}}^{2} / \varepsilon_{\mathrm{m}}^{2}\right) c_{\mathrm{d}} \alpha_{\mathrm{d}} / \varepsilon_{0}}{1+\frac{\alpha_{\mathrm{d}}}{4 \pi \varepsilon_{0}} \frac{L_{\mathrm{m}}^{2}}{\varepsilon_{\mathrm{m}}} \rho^{3 / 2} \xi_{0}} .
\end{aligned}
$$

Similar to the previous section, we can predict the shift in the plasmon resonance by the polarisability of the dye molecules, i.e. $\alpha_{\mathrm{d}}$. For the case where the molecules are isotropically in-plane, similar conditions applied:

- If $\Re\left\{\alpha_{\mathrm{d}}\left(\lambda_{\mathrm{PR}}\right)\right\}=0$, no shift in the plasmon resonance.

- If $0<\Re\left\{\alpha_{\mathrm{d}}\left(\lambda_{\mathrm{PR}}\right)\right\}<16 \pi \varepsilon_{0} \varepsilon_{\mathrm{m}} /\left(L_{\mathrm{m}}^{2} \rho^{3 / 2} \xi_{0}\right)$, the LSPR will be red-shifted.

- And if $\Re\left\{\alpha_{\mathrm{d}}\left(\lambda_{\mathrm{PR}}\right)\right\}<0$ or $\Re\left\{\alpha_{\mathrm{d}}\left(\lambda_{\mathrm{PR}}\right)\right\}>16 \pi \varepsilon_{0} \varepsilon_{\mathrm{m}} /\left(L_{\mathrm{m}}^{2} \rho^{3 / 2} \xi_{0}\right)$, then blue-shift will be expected.

For the radially oriented molecules, the conditions are:

- If $\Re\left\{\alpha_{\mathrm{d}}\left(\lambda_{\mathrm{PR}}\right)\right\}=0$, no shift in the plasmon resonance.

- If $\Re\left\{\alpha_{\mathrm{d}}\left(\lambda_{\mathrm{PR}}\right)\right\}>0$, it will result in red-shift in the plasmon resonance.

- And if $\Re\left\{\alpha_{\mathrm{d}}\left(\lambda_{\mathrm{PR}}\right)\right\}<0$, we shall expect the blue-shift.

With the parameters defining the polarisability of the Rhodamine $6 \mathrm{G}$ defined in Eq. 6.7, these conditions suggest that the radially oriented molecules will result in the plasmon resonance being red-shifted, regardless of the dye's concentration. However, that will not be the case for the isotropically in-plane molecules. According to the above conditions, we would expect the red-shift in the plasmon resonance for the dye's concentration $\rho$ up to the value of $7.68 \mathrm{~nm}^{-2}$ and for $\rho>7.68 \mathrm{~nm}^{-2}$, 


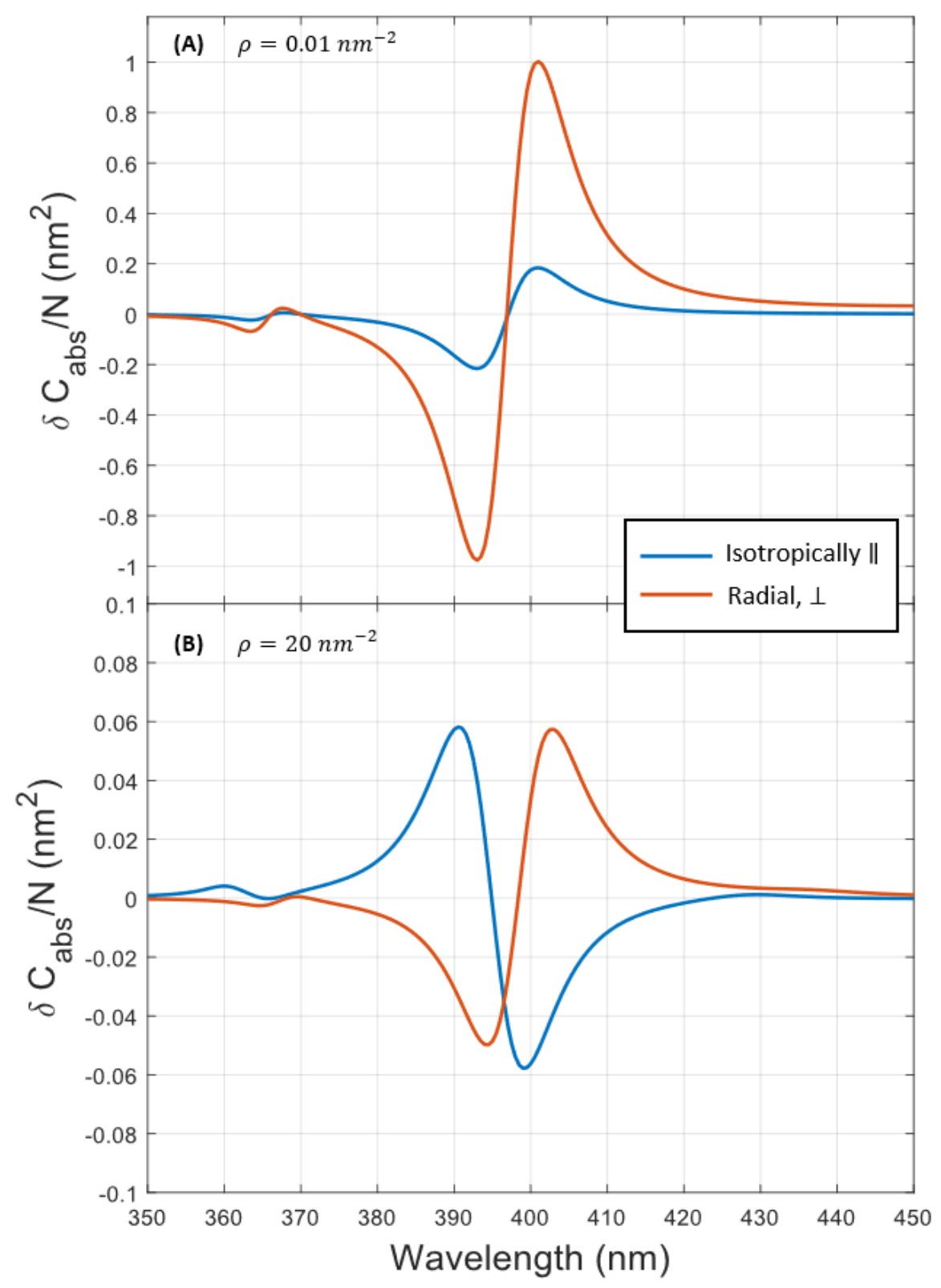

Figure 6.4: Normalised differential absorption cross-section of the two different orientations of dye-molecules, isotropically in-plane (radial) shown in blue (orange), showing for two different dye's concentrations, $\rho=0.01 \mathrm{~nm}^{-2}$ in (A) and $\rho=20 \mathrm{~nm}^{-2}$ in (B). 
the plasmon resonance will be blue-shifted. The demonstration of these is shown in Fig. 6.4. It shows that for $\rho=0.01 \mathrm{~nm}^{-2}$, both orientations of the dye result in red-shift in the plasmon resonance of the silver nano-particle (shown in Fig. 6.4A). In figure $6.4 \mathrm{~B}$, different shifts in the plasmon resonance are observed for the two orientations of the dye molecules (at $\rho=20 \mathrm{~nm}^{-2}$ ). For the radial orientation, the red-shift remain the same as for the lower concentration. However, for the isotropically in-plane case, the shift in the plasmon resonance has switched to blue-shift,

as expected (since $\rho>7.68 \mathrm{~nm}^{-2}$ ). At that high concentration, molecules are more likely to form dimers (or even trimers), which will make the situation even more complex to theoretically model. Therefore in many experiment with NPs size of 30 $\mathrm{nm}$ in radius, such concentration of the dye molecules is never considered.

Another feature we may discuss in Fig. 6.4 is the intensities differences of the two orientations. Figure $6.4 \mathrm{~A}$ indicates that the intensity in the differential absorption cross-section for the $\perp$ case is 5 times larger than that for the isotropically $\|$ case. This suggests that the shift in the plasmon resonance is 5 times stronger for the $\perp$ orientated dye-molecules than the isotropically in-plane orientation. Therefore, the plasmon resonance shift depends on the orientation of the dye-molecules, as well as their concentrations.

\subsection{Comparisons Between Mie Theory, GCDM, and COM- SOL}

So far, we have been using Mie theory to study the effect of the dye's orientation on the plasmon resonance. For the next study on the effect of the dye's location on the plasmon resonance, Mie theory will not be able to provide the solutions. Nevertheless, we still can use GCDM, as long as the nanoparticles are of spherical shapes. But, it is not a preferred method for the study of extension from spherical to non-spherical shaped-nanoparticles, as stated in the previous chapter and it is also too resource-intensive for multi-parameter studies. For this study, COMSOL would still be the ideal one among these.

In the previous chapter, we have presented the comparison between Mie theory and GCDM, and the results produced by COMSOL numerical simulations with Mie theory, at the electronic dye's resonance. We here will demonstrate similar results at the plasmon resonance to ensure the agreements between all calculations (Mie 
theory, GCDM, and COMSOL), by comparing them for an anisotropic layer of dyemolecules adsorbed fully around a silver nanoparticle.

\subsubsection{Full Core/Shell: Mie vs GCDM}

We first compare in Fig. 6.5, the results from Mie theory against the GCDM. The results compare the two theories for two different types of dye's orientations (for $\rho=0.01 \mathrm{~nm}^{-2}$ and $1.2 \mathrm{~nm}^{-2}$ ) adsorbed on the silver nanoparticle. The main dipolar plasmon resonance shift are clearly shown in both orientations (Fig. 6.5A and B) of the dye-molecules. In the case of isotropically in-plane molecules, the intensities in the normalised differential absorption cross-section are very close for the two values of $\rho$. The comparison to their corresponding results from the GCDM is rather difficult to observe any differences. However, these comparisons can be seen more clearly in the radial orientation case. In this figure, both Mie theory and the GCDM also predict the same shifts, even in the quadrupolar plasmon resonance for both orientations of the dye's molecules. This is the verification of the great agreement between the two theories.

\subsubsection{Full Core/shell: Mie vs. COMSOL}

The comparison between COMSOL simulations and Mie theory for the anisotropic shell of dye molecules adsorbed on an isotropic silver sphere is shown in Fig. 6.6, for a dye's concentration of $\rho=0.6 \mathrm{~nm}^{-2}$. Although, we cannot get the results at the quadrupolar resonance, COMSOL still provide accurate results for the anisotropic response of the dye-molecules on nanoparticle compared with Mie theory at the main dipolar plasmon resonance. From these figures 6.5 and 6.6, we are confident that our FEM implementation in COMSOL is capable of providing reliable results at both the dye's resonance and plasmon resonance. Therefore, we will confidentially use this to study how the dye's location on the nanoparticle's surface affect the plasmon resonance shift in the following section. 


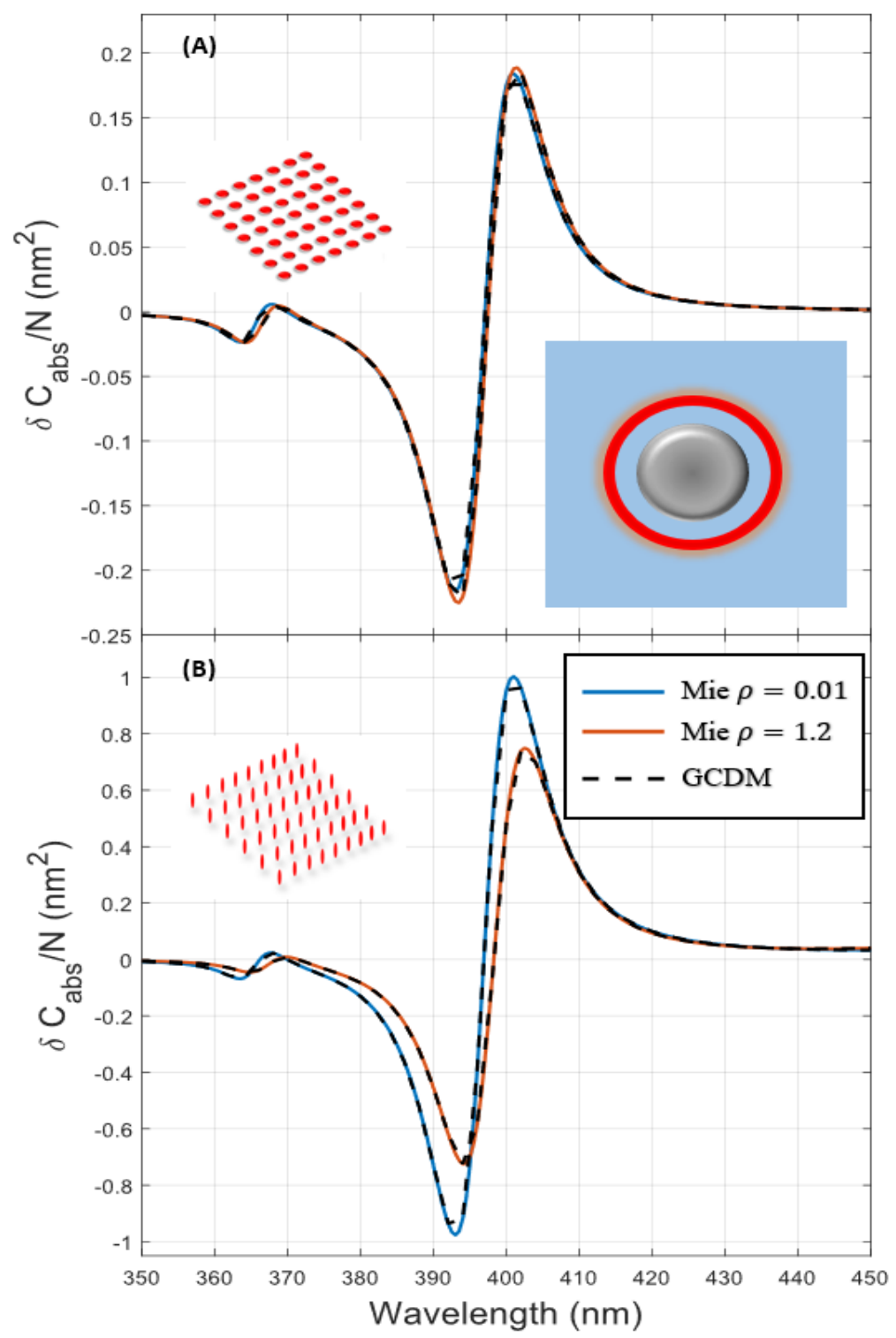

Figure 6.5: Comparison between Mie theory (solid lines) and GCDM (in dashed lines) for the normalised differential absorption cross-section of two different types of dye's orientations adsorbed on the silver nanoparticle of radius $14 \mathrm{~nm}$ embedded in water $\varepsilon_{\mathrm{m}}=$ $1.33^{2}$. The results are compared for two different $\rho, 0.01 \mathrm{~nm}^{-2}$ and $1.2 \mathrm{~nm}^{-2}$, showing isotropically in-plane dye-molecules in (A) and radially oriented molecules in (B). 


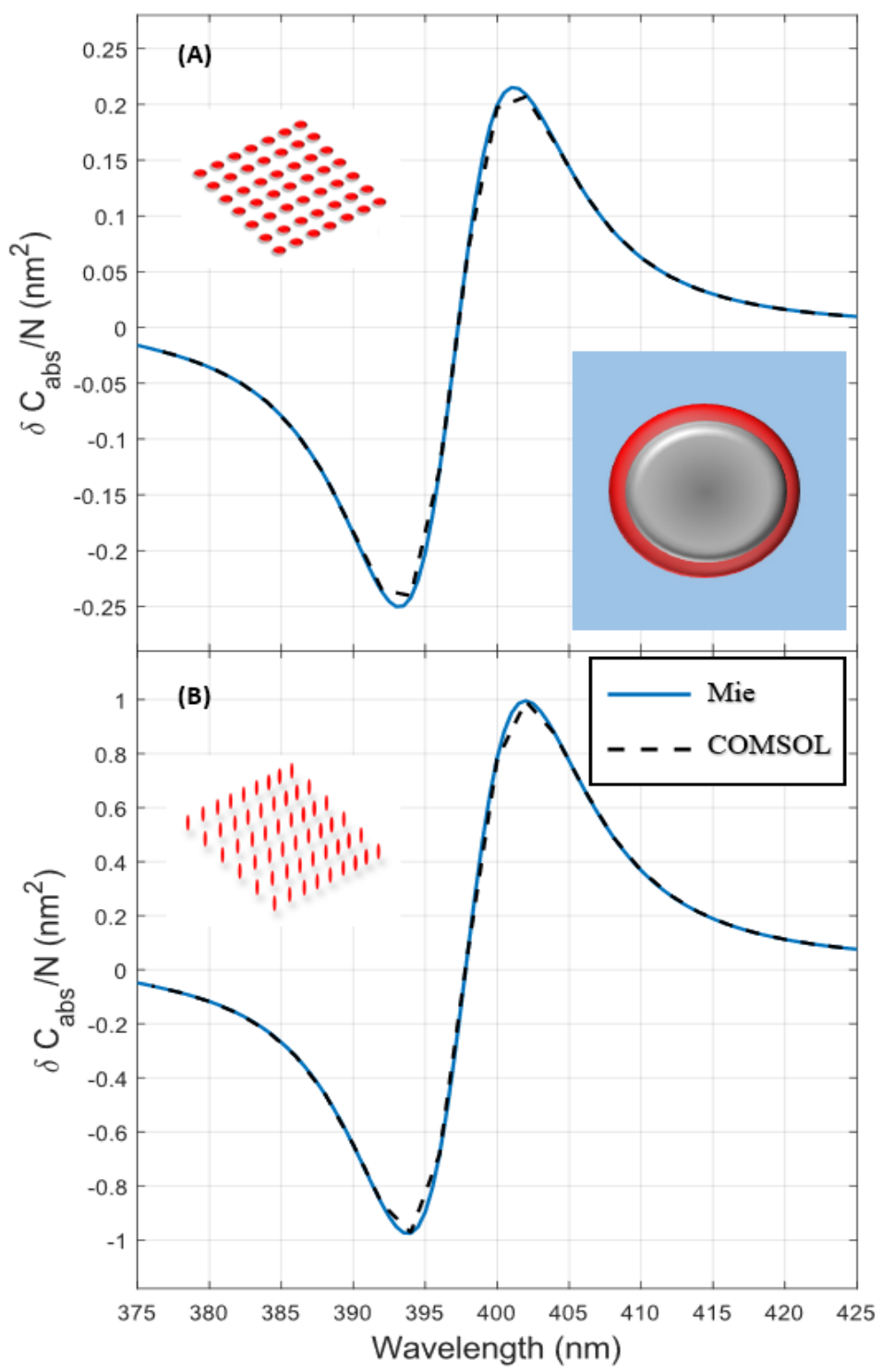

Figure 6.6: Comparison between Mie theory (solid lines) and COMSOL (in dashed lines) for the normalised differential absorption cross-section of two different types of dye's orientations adsorbed on the silver nanoparticle of radius $14 \mathrm{~nm}$ embedded in water $\varepsilon_{\mathrm{m}}=1.33^{2}$. The results are compared for two different $\rho=0.6 \mathrm{~nm}^{-2}$ showing isotropically in-plane dye-molecules in (A) and radially oriented molecules in (B). 


\subsection{Dye's Location Dependence}
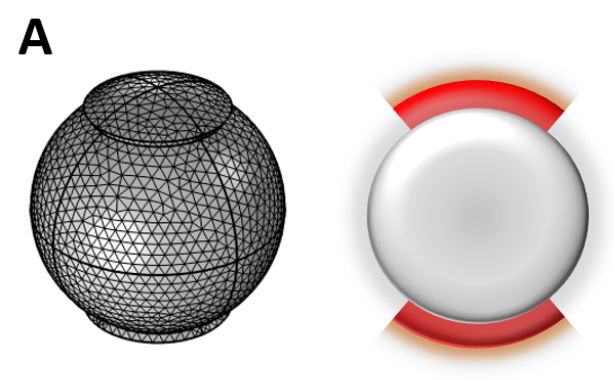

B
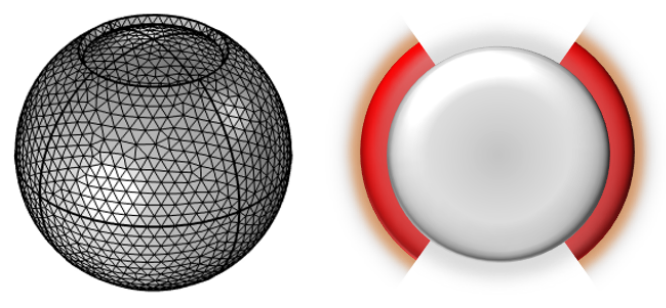

Figure 6.7: The geometry of the problem where the dye-molecules are adsorbed partially on the nanoparticle's surface showing in (A) top and bottom spherical caps [SC], and (B) partial spherical shell [PSS]. The PSS is the difference between the full spherical shell and the spherical caps (i.e., if the SC is set to be larger, the PSS will be smaller).

In this section, we will illustrate the effect of the dye's location on the plasmon resonance shift. In doing so, we will focus our problem on the spherical nanoparticle partially coated with anisotropic dye-molecules (extension to non-spherical particles can be easily followed but is not presented in this thesis). This will be separated into two cases, spherical caps on nanoparticle [SC] (Fig. 6.7A), and partial spherical shell around the nanoparticle [PSS] (Fig. 6.7B). These cases are different from the full spherical core-shell system. In the case of the full spherical core-shell system, altering the direction (or polarisation) of the plane-wave incident field will not affect the optical properties of the system. However, in the cases of SC and PSS adsorbed on the Ag-NP, the optical properties of these systems will definitely depend on the directions of the incident field (the situation will be more or less the same as for Spheroidal-NPs).

The results from the two cases of different locations of the dye-molecules adsorbed on the Ag-NP are summarised in Fig. 6.8. It shows the comparison between two different polarisations of the incident electric fields $\left[E_{x}, k_{z}\right.$ refers to $\left(\mathbf{E}_{\mathrm{Inc}}=\right.$ $\left.\mathbf{E}_{0} \exp \{-i k z\} \hat{\mathbf{e}}_{x}\right)$ and $E_{z}, k_{x}$ refers to $\left.\left(\mathbf{E}_{\text {Inc }}=\mathbf{E}_{0} \exp \{-i k x\} \hat{\mathbf{e}}_{z}\right)\right]$ for the SC adsorption on the left side of the graph (figures 6.8A and C) and PSS adsorption on the right-hand side (figures 6.8B and D). In both cases, we accounted for different orientations of the dye-molecules, isotropically in-plane and perpendicular to the Ag-NP's surface, on the top and bottom of the graph, respectively. 

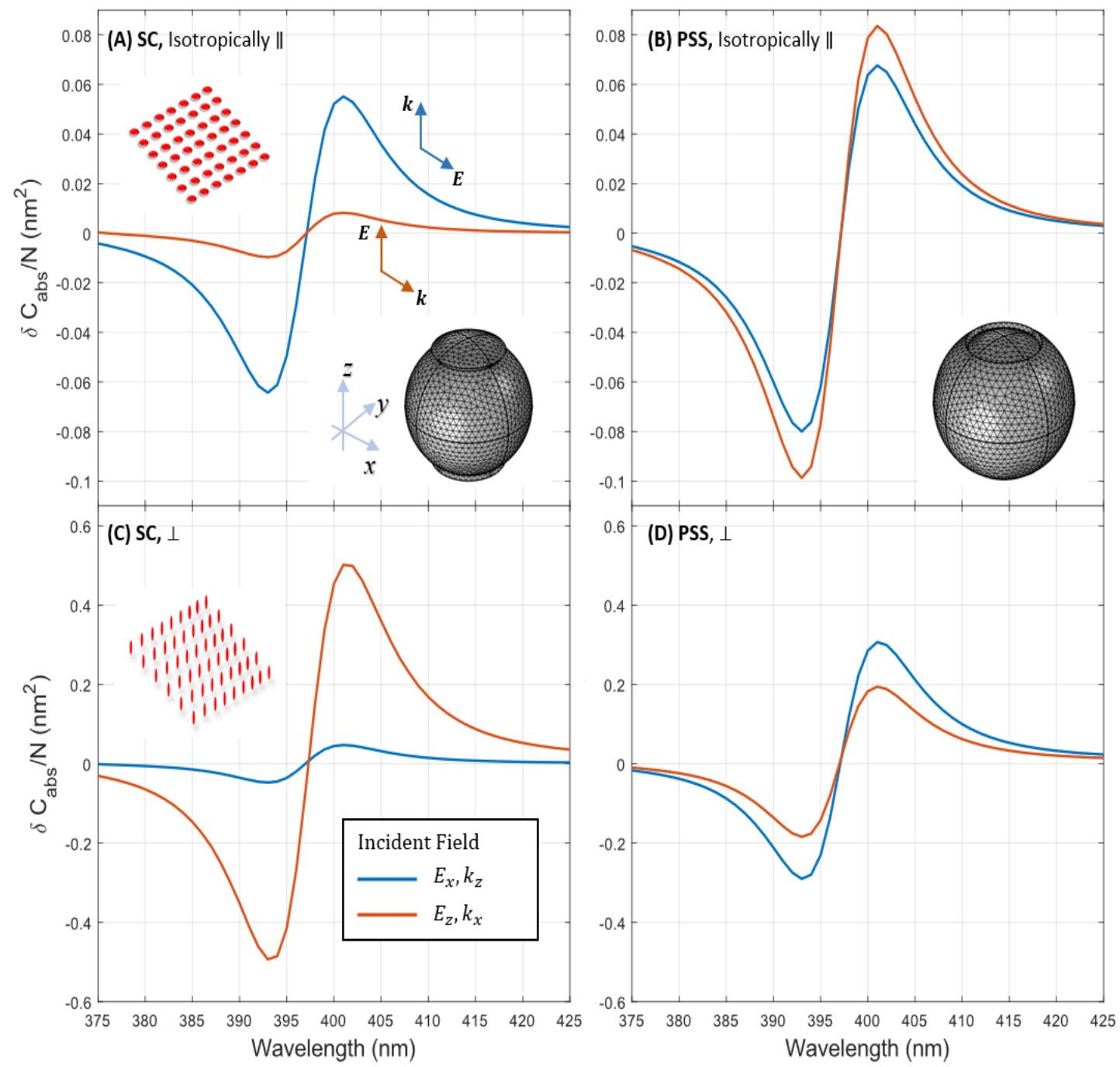

Figure 6.8: The spectra of normalised differential absorption cross-section for spherical caps [SC] of dye-molecules on Ag-NP (on the left), and partial spherical shell [PSS] on Ag-NP (on the right) showing the comparison between two different polarisations of the incident electric field (as shown in the legend). The top row of the graph, A and B (the bottom row, $\mathrm{C}$ and $\mathrm{D}$ ) correspond to the dye-molecules oriented isotropically in-plane (perpendicular) to the surface of the Ag-NP. The Ag-NP is of the size $14 \mathrm{~nm}$ in radius and the dye's surface coverage is of $\rho=0.6 \mathrm{~nm}^{-2}$. 


\subsubsection{Spherical Caps on Ag-NP}

We shall first focus and discuss the spectra on the left-hand side (figures 6.8A and $\mathrm{C}$ ) for the SC adsorption of the dye-molecules. For the isotropically in-plane molecules adsorbed on the Ag-NP, we observe a more enhanced shift in the plasmon resonance shift for the $E_{x}, k_{z}$ polarisation than the $E_{z}, k_{z}$ polarisation of the incident plane-wave excitation. In this case, the $E_{x}, k_{z}\left(E_{z}, k_{z}\right)$ polarisation corresponds to in-plane (out-of-plane) excitation of the field with respect to the orientation of the dye molecules. Therefore, we should expect the molecules to respond stronger for the in-plane excitation (more field enhancement at the plasmon resonance), i.e, larger shift in the plasmon resonance for $E_{x}, k_{z}$ than $E_{z}, k_{x}$ excitation ( $\sim 6.5$ times larger). For radially oriented molecules, they respond a lot stronger to the $E_{z}, k_{z}$ polarisation. As shown in figure $6.8 \mathrm{C}$, it is $\sim 15$ times stronger in the plasmon resonance shift for $E_{z}, k_{x}$ than $E_{x}, k_{z}$ polarisation.

At the dye's resonance (not shown here), the shift in the dye's resonance remains unchanged, regardless of the polarisations of the incident electric field. Different polarisation will only lead to different intensities (amplitudes) of the differential absorption cross-section.

\subsubsection{Partial Spherical Shell around Ag-NP}

The results for PSS adsorption are shown in figures 6.8B and D. There is not much of a difference in both cases of the polarisations and orientation of the dye-molecules. However, the responses of the dye-molecules to the polarisation of the incident electric field are different from the case of SC, as discussed in the previous section. This refers to the differences in the intensities of the blue and orange lines $\left(E_{x}, k_{z}\right.$ and $E_{z}, k_{x}$, respectively) in Fig. 6.8 .

We now discuss qualitatively the changes in the size of the PSS. If the PSS is getting larger (i.e. becoming a full spherical shell), the results of the differential absorption cross-section from the two different polarisations of the incident field will become indistinguishable, which mean they will result in the same shift in the plasmon resonance (for both orientations of the dye-molecules). However, if the PSS is getting smaller (i.e. becoming a thin ring around the Ag-NP), we would expect the plasmon resonance shift to be less for the $E_{x}, k_{z}$ polarisation when the dye-molecules are oriented isotropically in-plane, but it should not decrease as much for the $E_{z}, k_{x}$ polarisation. For the radial orientation, the opposite effect due to the 
polarisations of the electric field on the plasmon resonance shifts will be expected.

\subsection{Conclusion and Future Work}

We have demonstrated the effects which could influence the plasmon resonance of metallic nanoparticle (Ag-NP). For a single Ag-NP embedded in a medium, it is known that the plasmon resonance is shifted subject to the refractive index (or dielectric constant) of that medium being changed. Similarly, the plasmon resonance shift depends on the dielectric function of the dye molecules for the dye-coated AgNP. Here, we have shown that the orientations of the dye-molecules adsorbed on the NP are also affecting the shifts. The evidence shows that the plasmon resonance is being more red-shifted for the radial orientation than the isotropically in-plane orientation, for Rhodamine 6G adsorbed on Ag-NP. We also investigated how the locations of the dye-molecules adsorbed on the nanoparticle affect the shift in the plasmon resonance. The results suggested that the difference in the incident electric field polarisation also affect the plasmon resonance differently, depending on the locations of the dye-molecules. Therefore, in the study of how the locations of the dye-molecules adsorbed on nanoparticles may affect the plasmon resonance shift, the polarisation of the incident electric field is shown to be important and can affect the optical properties of the system.

The optical properties of a system consists of an anisotropic molecule (or more) closely attached to noble NPs (silver or gold) or specially engineered NPs (like Janus particles) is one of the applications for this work. Janus particles are the special types of NPs whose surfaces have more than one distinct physical properties. Therefore, when a molecule is attached to its surface, the optical properties of the whole system will significantly depend on the location of the molecule on its surface. By making use of this study, one will be able to determine which orientation and where the molecule should be attached to the surface of the NPs to give the strongest response or highest field enhancements. These could have major implications for sensing applications, where a certain orientation and location of the molecules matter.

Overall, our implementation of FEM in COMSOL seems to provide a great understanding of how the locations of dye molecules on the spherical nanoparticle's surface affect the surface plasmon resonance. It is predictable, yet not obvious, that similar results will be observed for the case of the non-spherical nanoparticle. How- 
ever, our prediction is actually based on the results in the previous Chapters 3 and 4. Among all the results shown in this thesis, the plasmon resonance and the dye's resonance are well separated, which made the study on the interactions between them not as complicated. But in the case where the two resonances are inseparable (i.e. strong resonance couplings), it will surely be complicated to interpret the resulting outcome of the plasmon-dye interactions. Therefore, further theoretical investigations are needed in order to address this issue. 


\section{Conclusion and Outlook}

The work presented in this thesis shows how we can computationally model the optical properties of nanoparticles coated with anisotropic dye-molecules by defining a refined effective medium model (EMM), which is a combination of anisotropic Mie theory and the effective dielectric functions for anisotropic media. This new refined EMM overcomes the GCDM's problems: high computational cost and complexity, and it has comparable predictive power as the GCDM. We also show how we can extend this to model the optical properties of non-spherical shaped coated nanoparticles by using COMSOL simulations.

In Chapter 4, we demonstrated good agreement between the predictions of our refined EMM and the GCDM. The best agreement between the two models is when the dye molecules are oriented radially to the nanoparticle's surface. In the case of randomly in-plane orientations, the GCDM predictions contain the information about the broadening in the dye's resonance spectra (for higher coverage, $\rho$ ). However, this broadening effect does not show in the refined EMM predictions. This is because the derived effective dielectric functions do not account for the random inplane orientation, instead it replaces all dipoles by isotropically in-plane polarisable smarties-like dipoles. Therefore, the information about the broadening, which is due to the dipole pointing in different directions is lost. However, the optical response of molecules on metal surfaces is typically dominated by the perpendicular component, as evidenced by the fact that the differential absorption cross-sections are much larger for the perpendicular case than for the in-plane orientations. Therefore, the errors observed between the GCDM and the refined EMM will not affect the intermediate orientations, where even a small response in the perpendicular component is present. We have so far modelled our refined EMM with the dye molecules' polarisability as a single peak from a single Lorentz oscillation model, whereas experimentally, there are two prominent absorption peaks (for Rhodamine $6 \mathrm{G}$ ) to be included. Another aspect that may improve our refined EMM to predict experimental results is the formation of dimers of the dye molecules. 
From here, future work could consist of the study, where we include both the two prominent absorption peaks of Rhodamine $6 \mathrm{G}$ and the effect of the dimers formations on the overall optical properties of the whole system. The required information for this study would be: (i) the conditions of when the dye-molecules can form dimers? (ii) how do we account for dimers' effects (different forms of dimers) in our EMM, i.e. the polarisability, or the effective dielectric functions? and (ii) with all of these being taken into consideration, how well will it predict the experimental results compared to the GCDM?

In Chapter 5, we showed that the extension of the light scattering study from spherical to non-spherical objects can be done by implementing FEM into the COMSOL simulations. To ensure that COMSOL correctly accounts for the anisotropic effect in the shell medium, we showed that the basis transformation from spherical and spheroidal to Cartesian coordinates are necessary. We validated the results from COMSOL by comparing them to the Mie theory for spherical coated nanoparticle. We then used a similar implementation for the spheroidal coated nanoparticle. The results suggest that the shifts in the dye's resonance depend on the polarisation of the incident electric field for the spheroidal coated nanoparticle, which is due to the shape of the particle and the orientations of the dye-molecules. However, many spectra from the theoretical modelling in Chapters 3 to 5 are presented at the dye's resonance. This only provides information about the effect of the nanoparticle has on the optical properties of the dyes molecules. The opposite effect should, nevertheless, be considered as well. We demonstrate this in Chapter 6, where the investigation of how the localised surface plasmon resonance of a noble metal (i.e. silver nanosphere) may be affected by the refractive indices of the surrounding media. The results from our theoretical modelling in Chapter 6 suggest that the plasmon resonance of the Ag nanosphere is strongly dependent on the properties of the dye-molecules, their orientations, their locations on the surface of the nanoparticle, and the polarisation of the incident electric field.

It is noteworthy that the results shown in Chapter 6 are only for the nanosphere. Therefore, further investigation is needed for non-spherical cases. Generally, for spheroidal particles, there are two plasmon resonances induced by different polarisations of the incident electric field. These two plasmon resonances depend on the aspect ratio $h$, i.e. shape and size of the spheroid. In the presence of the dyemolecules being adsorbed on its surface, this system will induce different interactions 
between the dye molecules and the plasmonic structure depending on the incident electric field. In that case, the effect of the anisotropic dye molecules on the long and short resonances of the nanoparticle could be interesting to investigate. Moreover, these could also lead to the study of the strong plasmon-molecule coupling at the nanoscale, where the resonances are proximate. Emerging applications could be found in quantum and nonlinear optics, as well as opportunities for modifying material-related properties and/or meta-materials.

The work that we demonstrate in this thesis is believed to find application in many areas relating to the optical properties of dye molecules on metallic nanoparticles (for example, sensing), including molecular plasmonic (at both weak and strong coupling regimes) and SERS, where the orientation, surface coverage, and distance to the particle's surface of the adsorbates can have large effects.

The spectroscopic study of chiroptical effects at the nanoscale has seen a resurgence in the past few years, with many works shedding new insights about the physics that govern the interaction of circularly polarised light with chiral organic molecules, bio-synthetic and synthetic nanomaterials. Therefore, in addition to our conclusion above, we also believe that this work could be a useful tool to study the chirality and circular dichroism properties of systems consisting of dyes partially coated on nanospheres or nanorods, arranged in a chiral geometry. These types of research could potentially find application in other exciting fields, such as the study of biomolecules and biopharmaceutical industry. 



\section{A. Bessel Function Series Expressions}

The regular and complex-ordered Riccati-Bessel functions may be written in a power series as ([90], sections 9.1.2 and 9.1.10)

$$
\begin{aligned}
& \psi_{n}(x)=x^{n+1} \sum_{k=0}^{\infty} \frac{\left(-\frac{1}{2}\right)^{k}}{k !} a_{k, n} x^{2 k} \\
& \chi_{n}(x)=\frac{-1}{x^{n}} \sum_{k=0}^{\infty} \frac{\left(-\frac{1}{2}\right)^{k}}{k !} b_{k, n} x^{2 k}
\end{aligned}
$$

where the coefficient $a$ and $b$ are defined as (NB: for complex-ordered Bessel function, $n$ will be replaced by $w$ defined in Eq. 3.11)

$$
\begin{aligned}
a_{k, n} & =\frac{1}{(2 n+1) ! !} \prod_{j=1, \text { odd }}^{2 k-1} \frac{1}{2 n+j+2} \\
& =\frac{1}{(2 n+2 k+1) ! !} \\
b_{k, n} & =(2 n-1) ! ! \prod_{j=1, \text { odd }}^{2 k-1} \frac{1}{j-2 n} \\
& = \begin{cases}(-1)^{k}(2 n-2 k-1) ! ! & \text { for } k \leq n \\
\frac{(-1)^{n}}{(2 k-2 n-1) ! !}, & \text { for } k>n .\end{cases}
\end{aligned}
$$

The notation $(2 l+1)$ !! is the double factorial operation, defined as:

$$
(2 l+1) ! !=(2 l+1) \times(2 l-1) \times(2 l-3) \times \ldots \times 5 \times 3 \times 1=\frac{(2 l+1) !}{2 \times l !} .
$$

For regular Bessel functions, we can use the Gamma function to compute these series. However, for the Bessel functions of complex-orders, the domains of the Gamma functions will be complex and regular Gamma functions will fail to give the results. But with a new routine in MatLab using Lanczos series approximation, the complex Gamma function can be computed. Therefore, we can make use of this to calculated our Bessel functions of complex-orders. 
The derivative of these Raccati-Bessel functions can be expressed as:

$$
\begin{aligned}
& \psi_{n}^{\prime}(x)=x^{n} \sum_{k=0}^{\infty} \frac{\left(-\frac{1}{2}\right)^{k}(2 k+n+1)}{k !} a_{k, n} x^{2 k}, \\
& \chi_{n}^{\prime}(x)=\frac{-1}{x^{n+1}} \sum_{k=0}^{\infty} \frac{\left(-\frac{1}{2}\right)^{k}(2 k-n)}{k !} b_{k, n} x^{2 k},
\end{aligned}
$$

The spherical Bessel functions are related to these Riccati-Bessel functions by:

$$
\begin{array}{r}
j_{n}(x)=\psi_{n}(x) / x \\
y_{n}(x)=\chi_{n}(x) / x,
\end{array}
$$

and the Bessel functions can be found as:

$$
\begin{aligned}
J_{n+1 / 2}(x) & =\sqrt{\frac{2 x}{\pi}} j_{n}(x) \\
Y_{n+1 / 2}(x) & =\sqrt{\frac{2 x}{\pi}} y_{n}(x) .
\end{aligned}
$$




\section{B. Associated Legendre Function}

The associated Legendre function is a solution to the differential equation (as from [52], Eq. 8.1.1)

$$
\left(1-x^{2}\right) \frac{d^{2} y}{d x^{2}}-2 x \frac{d y}{d x}+\left[n(n+1)-\frac{m^{2}}{1-x^{2}}\right] y=0
$$

where we are interested in solutions

$$
y=P_{n}^{m}(\cos \theta)
$$

where $m$ is the order of the solutions, $n$ is the degree, and we use $x=\cos \theta$. Cases where $m=0$ are known as Legendre Polynomials, $P_{n}(x)$. We can express the associated Legendre functions in terms of the Legendre polynomial as (for $m \geq 0$ )

$$
P_{n}^{m}(x)=(-1)^{m}\left(1-x^{2}\right)^{m / 2} \frac{d^{m}}{d x^{m}} P_{n}(x),
$$

where the Legendre polynomial is given by the expression:

$$
P_{n}(x)=\frac{1}{2^{n} n !} \frac{d^{n}}{d x^{n}}\left(x^{2}-1\right)^{n} .
$$

The factor $(-1)^{m}$ in the definition of Eq. B.3 is known as the Condon-Shortley phase. In the case of negative order $m$, the expression for the associated Legendre function is

$$
P_{n}^{-m}(x)=(-1)^{m} \frac{(n-m) !}{(n+m) !} P_{n}^{m}(x) .
$$





\section{Orthogonal Properties of VSHs}

We here will use known results of VSHs for solutions to the Maxwell equations (for a light scattering problem by a isotropic sphere) in spherical coordinates. The solution to wave-vector $\psi$ is found to be:

$$
\psi_{n m}(r, \theta, \phi)=\sqrt{\frac{2 n+1}{4 \pi} \frac{(n-m) !}{(n+m) !} \frac{1}{n(n+1)}} z_{n}(k r) P_{n}^{m}(\cos \theta) e^{i m \phi}
$$

We will keep in mind the factor, but will leave left out of the following expressions. The VSHs, $\mathbf{M}_{n m}$ and $\mathbf{N}_{n m}$ are defined as:

$$
\begin{gathered}
\mathbf{M}_{n m}=\nabla \times\left(\mathbf{r} \psi_{n m}\right)=\left[\begin{array}{c}
0 \\
\frac{i m}{\sin \theta} z_{n}(\rho) P_{n}^{m}(\cos \theta) e^{i m \phi} \\
-z_{n}(\rho) \frac{d P_{n}^{m}(\cos \theta)}{d \theta} e^{i m \phi}
\end{array}\right] \\
\mathbf{N}_{n m}=\frac{\nabla \times \mathbf{M}_{n m}}{k}=\left[\begin{array}{c}
n(n+1) \frac{z_{n}(\rho)}{\rho} P_{n}^{m}(\cos \theta) e^{i m \phi} \\
\frac{1}{\rho} \frac{d}{d \rho}\left[\rho z_{n}(\rho)\right] \frac{d P_{n}^{m}(\cos \theta)}{d \theta} e^{i m \phi} \\
i m \frac{P_{n}^{m}(\cos \theta)}{\sin \theta} \frac{1}{\rho} \frac{d}{d \rho}\left[\rho z_{n}(\rho)\right] e^{i m \phi}
\end{array}\right] .
\end{gathered}
$$

The VSHs, $\mathbf{M}_{n m}$ and $\mathbf{N}_{n m}$, form a complete orthogonal basis for the divergence-less solutions of the wave vector equation (and therefore of Maxwell's equations in the absence of sources). The orthogonality is related to that of spherical harmonics and concerns the angular dependence only. More specifically, we have (we shall use the following results without proofs):

$$
\begin{array}{r}
\iint \mathbf{M}_{n m}^{(i)}(k, r, \Omega) \cdot\left[\mathbf{N}_{n^{\prime} m^{\prime}}^{(j)}(k, r, \Omega)\right]^{*} d \Omega=0 \\
\forall\left(m, m^{\prime}, n, n^{\prime} ;(i, j)=1 . .4\right),
\end{array}
$$


where $*$ denotes the complex conjugate, $\Omega=(\theta, \phi), d \Omega=\sin \theta d \theta d \phi$, and $\iint=$ $\int_{\theta=0}^{\phi} \int_{\phi=0}^{2 \pi}$. We also have,

$$
\iint \mathbf{M}_{n m}^{(i)}(k, r, \Omega) \cdot\left[\mathbf{M}_{n^{\prime} m^{\prime}}^{(j)}(k, r, \Omega)\right]^{*} d \Omega=\delta_{n, n^{\prime}} \delta_{m, m^{\prime}} z_{n}^{(i)}(k r)\left[z_{n}^{(j)}(k r)\right]^{*}
$$

and,

$$
\begin{aligned}
& \iint \mathbf{M}_{n m}^{(i)}(k, r, \Omega) \cdot\left[\mathbf{M}_{n^{\prime} m^{\prime}}^{(j)}(k, r, \Omega)\right]^{*} d \Omega=\delta_{n, n^{\prime}} \delta_{m, m^{\prime}} \times \\
& \\
& {\left[n(n+1) \frac{z_{n}^{(i)}(k r)\left[z_{n}^{(j)}(k r)\right]^{*}}{|k r|^{2}}+\frac{\left[z_{n}^{(i)}(k r)+k r z_{n}^{(i)}(k r)\right]\left[z_{n}^{(j)}(k r)+k r z_{n}^{(j)}(k r)\right]^{*}}{|k r|^{2}}\right] .}
\end{aligned}
$$

These orthogonality relations ate important, and for example useful for determining the coefficients of the expansion of a given electric field in a sum of VSHs. Here are additional useful expressions,

$$
\begin{gathered}
\iint\left[\begin{array}{l}
\left.\mathbf{M}_{n m}^{(i)}(k, r, \Omega) \times\left[\mathbf{M}_{n^{\prime} m^{\prime}}^{(j)}(k, r, \Omega)\right]^{*}\right] \\
\mathbf{N}_{n m}^{(i)}(k, r, \Omega) \times\left[\mathbf{N}_{n^{\prime} m^{\prime}}^{(j)}(k, r, \Omega)\right]^{*}
\end{array}\right] \cdot \hat{\mathbf{e}}_{r} d \Omega=0 \\
\iint \mathbf{M}_{n m}^{(i)}(k, r, \Omega) \times\left[\mathbf{N}_{n^{\prime} m^{\prime}}^{(j)}(k, r, \Omega)\right]^{*} \cdot \hat{\mathbf{e}}_{r} d \Omega=\delta_{n, n^{\prime}} \delta_{m, m^{\prime}} \times \\
\frac{z_{n}^{(i)}(k r)}{k r}\left[z_{n}^{(j)}(k r)+k r z_{n}^{(j)}(k r)\right]^{*} .
\end{gathered}
$$

For anisotropic VSHs, these above orthogonal properties still hold. We only need to change from $z_{n}$, the regular Bessel functions to $\tilde{z}_{n}$, the Bessel functions of complex-orders. 


\section{Solutions for 2-Layer System}

\section{D.1 Full Anisotropic Mie Solutions}

We shall first write down the fields expansions in terms of VSHs as follows,

$$
\begin{aligned}
& \mathbf{E}_{\mathrm{Inc}}=E_{0} \sum_{n, m} a_{n m} \mathbf{M}_{n m}^{(1)}\left(k_{\mathrm{m}} \mathbf{r}\right)+b_{n m} \mathbf{N}_{n m}^{(1)}\left(k_{\mathrm{m}} \mathbf{r}\right) \\
& \mathbf{H}_{\mathrm{Inc}}=\frac{k_{\mathrm{m}}}{i \omega \mu} E_{0} \sum_{n, m} a_{n m} \mathbf{N}_{n m}^{(1)}\left(k_{\mathrm{m}} \mathbf{r}\right)+b_{n m} \mathbf{M}_{n m}^{(1)}\left(k_{\mathrm{m}} \mathbf{r}\right) \\
& \mathbf{E}_{\mathrm{Sca}}=E_{0} \sum_{n, m} p_{n m} \mathbf{M}_{n m}^{(3)}\left(k_{\mathrm{m}} \mathbf{r}\right)+q_{n m} \mathbf{N}_{n m}^{(3)}\left(k_{\mathrm{m}} \mathbf{r}\right) \\
& \mathbf{H}_{\mathrm{Sca}}=\frac{k_{\mathrm{m}}}{i \omega \mu} E_{0} \sum_{n, m} p_{n m} \mathbf{N}_{n m}^{(3)}\left(k_{\mathrm{m}} \mathbf{r}\right)+q_{n m} \mathbf{M}_{n m}^{(3)}\left(k_{\mathrm{m}} \mathbf{r}\right) \\
& \mathbf{E}_{\mathrm{C}}=E_{0} \sum_{n, m} c_{n m} \mathbf{M}_{n m}^{(1)}\left(k_{\mathrm{in}} \mathbf{r}\right)+d_{n m} \mathbf{N}_{n m}^{(1)}\left(k_{\mathrm{in}} \mathbf{r}\right) \\
& \mathbf{H}_{\mathrm{C}}=\frac{k_{\mathrm{c}}}{i \omega \mu} E_{0} \sum_{n, m} c_{n m} \mathbf{N}_{n m}^{(1)}\left(k_{\mathrm{in}} \mathbf{r}\right)+d_{n m} \mathbf{M}_{n m}^{(1)}\left(k_{\mathrm{in}} \mathbf{r}\right) \\
& \mathbf{E}_{\mathrm{Sh}}=\frac{\varepsilon_{\varepsilon_{\mathrm{t}}}}{E_{0}} \sum_{n, m} \alpha_{n m} \mathbf{M}_{n m}^{(1)}\left(k_{\mathrm{t}} \mathbf{r}\right)+\beta_{n m} \tilde{\mathbf{N}}_{n m}^{(1)}\left(k_{\mathrm{t}} \mathbf{r}\right)+\gamma_{n m} \mathbf{M}_{n m}^{(2)}\left(k_{\mathrm{t}} \mathbf{r}\right)+\delta_{n m} \tilde{\mathbf{N}}_{n m}^{(2)}\left(k_{\mathrm{t}} \mathbf{r}\right) \\
& \mathbf{H}_{\mathrm{Sh}}=\frac{k_{\mathrm{t}}}{i \omega \mu} E_{0} \sum_{n, m} \alpha_{n m} \mathbf{N}_{n m}^{(1)}\left(k_{\mathrm{t}} \mathbf{r}\right)+\beta_{n m} \tilde{\mathbf{M}}_{n m}^{(1)}\left(k_{\mathrm{t}} \mathbf{r}\right)+\gamma_{n m} \mathbf{N}_{n m}^{(2)}\left(k_{\mathrm{t}} \mathbf{r}\right)+\delta_{n m} \tilde{\mathbf{M}}_{n m}^{(2)}\left(k_{\mathrm{t}} \mathbf{r}\right) \\
& \mathbf{E}_{\mathrm{Bet}}=E_{0} \sum_{n, m} e_{n m} \mathbf{M}_{n m}^{(1)}\left(k_{\mathrm{m}} \mathbf{r}\right)+f_{n m} \mathbf{N}_{n m}^{(1)}\left(k_{\mathrm{m}} \mathbf{r}\right)+g_{n m} \mathbf{M}_{n m}^{(2)}\left(k_{\mathrm{m}} \mathbf{r}\right)+h_{n m} \mathbf{N}_{n m}^{(2)}\left(k_{\mathrm{m}} \mathbf{r}\right) \\
& \mathbf{H}_{\mathrm{Bet}}=\frac{k_{\mathrm{m}}}{i \omega \mu} E_{0} \sum_{n, m} e_{n m} \mathbf{N}_{n m}^{(1)}\left(k_{\mathrm{m}} \mathbf{r}\right)+f_{n m} \mathbf{M}_{n m}^{(1)}\left(k_{\mathrm{m}} \mathbf{r}\right)+g_{n m} \mathbf{N}_{n m}^{(2)}\left(k_{\mathrm{m}} \mathbf{r}\right)+h_{n m} \mathbf{M}_{n m}^{(2)}\left(k_{\mathrm{m}} \mathbf{r}\right) .
\end{aligned}
$$


Then we can apply the boundary conditions, similar to Eqs. 3.17 - 3.24. At $r=a$, the boundary conditions are:

$$
\begin{aligned}
& \mathbf{E}_{\text {Bet }} \cdot \hat{\theta}=\mathbf{E}_{\text {in }} \cdot \hat{\theta} \\
& \mathbf{E}_{\text {Bet }} \cdot \hat{\phi}=\mathbf{E}_{\text {in }} \cdot \hat{\phi} \\
& \mathbf{H}_{\text {Bet }} \cdot \hat{\theta}=\mathbf{H}_{\text {in }} \cdot \hat{\theta} \\
& \mathbf{H}_{\text {Bet }} \cdot \hat{\phi}=\mathbf{H}_{\text {in }} \cdot \hat{\phi} .
\end{aligned}
$$

At $r=b$, the boundary conditions are:

$$
\begin{aligned}
& \mathbf{E}_{\text {Aniso }} \cdot \hat{\theta}=\mathbf{E}_{\text {Bet }} \cdot \hat{\theta} \\
& \mathbf{E}_{\text {Aniso }} \cdot \hat{\phi}=\mathbf{E}_{\text {Bet }} \cdot \hat{\phi} \\
& \mathbf{H}_{\text {Aniso }} \cdot \hat{\theta}=\mathbf{H}_{\text {Bet }} \cdot \hat{\theta} \\
& \mathbf{H}_{\text {Aniso }} \cdot \hat{\phi}=\mathbf{H}_{\text {Bet }} \cdot \hat{\phi} .
\end{aligned}
$$

At $r=c$, the boundary conditions are:

$$
\begin{array}{r}
\left(\mathbf{E}_{\text {Inc }}+\mathbf{E}_{\text {Sca }}\right) \cdot \hat{\theta}=\mathbf{E}_{\text {Aniso }} \cdot \hat{\theta} \\
\left(\mathbf{E}_{\text {Inc }}+\mathbf{E}_{\text {Sca }}\right) \cdot \hat{\phi}=\mathbf{E}_{\text {Aniso }} \cdot \hat{\phi} \\
\left(\mathbf{H}_{\text {Inc }}+\mathbf{H}_{\text {Sca }}\right) \cdot \hat{\theta}=\mathbf{H}_{\text {Aniso }} \cdot \hat{\theta} \\
\left(\mathbf{H}_{\text {Inc }}+\mathbf{H}_{\text {Sca }}\right) \cdot \hat{\phi}=\mathbf{H}_{\text {Aniso }} \cdot \hat{\phi} .
\end{array}
$$

Applying these boundary conditions will lead to, at $r=a$

$$
\begin{aligned}
& e_{n m} \psi_{n}(z)+g_{n m} \chi_{n}(z)=c_{n m} \psi_{n}\left(s_{\mathrm{in}} z\right) / s_{\mathrm{in}} \\
& f_{n m} \psi_{n}^{\prime}(z)+h_{n m} \chi_{n}^{\prime}(z)=d_{n m} \psi_{n}^{\prime}\left(s_{\mathrm{in}} z\right) / s_{\mathrm{in}} \\
& e_{n m} \psi_{n}^{\prime}(z)+g_{n m} \chi_{n}^{\prime}(z)=c_{n m} \psi_{n}^{\prime}\left(s_{\mathrm{in}} z\right) \\
& f_{n m} \psi_{n}(z)+h_{n m} \chi_{n}(z)=d_{n m} \psi_{n}\left(s_{\mathrm{in}} z\right),
\end{aligned}
$$

where $s_{\text {in }}=\sqrt{\varepsilon_{\text {in }}} / \sqrt{\varepsilon_{\mathrm{m}}}, z=k_{\mathrm{m}} a$. And these 4 equations lead to:

$$
\begin{aligned}
& \frac{g_{n m}}{e_{n m}}=-\frac{s_{\text {in }} \psi_{n}(z) \psi_{n}^{\prime}\left(s_{\text {in }} z\right)-\psi_{n}(z) \psi_{n}^{\prime}\left(s_{\text {in }} z\right)}{s_{\text {in }} \chi_{n}(z) \psi_{n}^{\prime}\left(s_{\text {in }} z\right)-\chi_{n}(z) \psi_{n}^{\prime}\left(s_{\text {in }} z\right)} \equiv \Gamma_{n}^{(1)} \\
& \frac{h_{n m}}{f_{n m}}=-\frac{s_{\text {in }} \psi_{n}^{\prime}(z) \psi_{n}\left(s_{\text {in }} z\right)-\psi_{n}^{\prime}(z) \psi_{n}\left(s_{\text {in }} z\right)}{s_{\text {in }} \chi_{n}^{\prime}(z) \psi_{n}\left(s_{\text {in }} z\right)-\chi_{n}^{\prime}(z) \psi_{n}\left(s_{\text {in }} z\right)} \equiv \Delta_{n}^{(1)} .
\end{aligned}
$$

Boundary condition, at $r=b$

$$
\begin{aligned}
& {\left[\alpha_{n m} \psi_{n}\left(s_{\mathrm{t}} y\right)+\gamma_{n m} \chi_{n}\left(s_{\mathrm{t}} y\right)\right] / s_{\mathrm{t}}=e_{n m} \psi_{n}(y)+g_{n m} \chi_{n}(y)} \\
& {\left[\beta_{n m} \tilde{\psi}_{n}^{\prime}\left(s_{\mathrm{t}} y\right)+\delta_{n m} \tilde{\chi}_{n}^{\prime}\left(s_{\mathrm{t}} y\right)\right] / s_{\mathrm{t}}=f_{n m} \psi_{n}^{\prime}(y)+h_{n m} \chi_{n}^{\prime}(y)} \\
& \alpha_{n m} \psi_{n}^{\prime}\left(s_{\mathrm{t}} y\right)+\gamma_{n m} \chi_{n}^{\prime}\left(s_{\mathrm{t}} y\right)=e_{n m} \psi_{n}^{\prime}(y)+g_{n m} \chi_{n}^{\prime}(y) \\
& \beta_{n m} \tilde{\psi}_{n}\left(s_{\mathrm{t}} y\right)+\delta_{n m} \tilde{\chi}_{n}\left(s_{\mathrm{t}} y\right)=f_{n m} \psi_{n}(y)+h_{n m} \chi_{n}(y),
\end{aligned}
$$


where $s_{\mathrm{t}}=\sqrt{\varepsilon_{\mathrm{t}}} / \sqrt{\varepsilon_{\mathrm{m}}}, y=k_{\mathrm{m}} b$. And these equations lead to:

$$
\begin{aligned}
\frac{\gamma_{n m}}{\alpha_{n m}} & =-\frac{[\psi \psi]_{n, y}^{m, s}+\Gamma_{n}^{(1)}[\psi \chi]_{n, y}^{m, s}}{[\chi \psi]_{n, y}^{m, s}+\Gamma_{n}^{(1)}[\chi \chi]_{n, y}^{m, s}} \equiv \Gamma_{n}^{(2)} \\
\frac{\delta_{n m}}{\beta_{n m}} & =-\frac{[\psi \psi]_{n, y}^{e, s}+\Delta_{n}^{(1)}[\psi \chi]_{n, y}^{e, s}}{[\chi \psi]_{n, y}^{e, s}+\Delta_{n}^{(1)}[\chi \chi]_{n, y}^{e, s}} \equiv \Gamma_{n}^{(2)} .
\end{aligned}
$$

Boundary condition, at $r=c\left[x=k_{\mathrm{m}} c\right]$ :

$$
\begin{aligned}
& a_{n m} \psi_{n}(x)+p_{n m} \xi_{n}(x)=\left[\alpha_{n m} \psi_{n}\left(s_{\mathrm{t}} x\right)+\gamma_{n m} \chi_{n}\left(s_{\mathrm{t}} x\right)\right] / s_{\mathrm{t}} \\
& b_{n m} \psi_{n}^{\prime}(x)+q_{n m} \xi_{n}^{\prime}(x)=\left[\beta_{n m} \tilde{\psi}_{n}^{\prime}\left(s_{\mathrm{t}} x\right)+\delta_{n m} \tilde{\chi}_{n}^{\prime}\left(s_{\mathrm{t}} x\right)\right] / s_{\mathrm{t}} \\
& \left.a_{n m} \psi_{n}^{\prime}(x)+p_{n m} \xi_{n}^{\prime}(x)=\alpha_{n m} \psi_{n}^{\prime}\left(s_{\mathrm{t}} x\right)+\gamma_{n m} \chi_{n}^{\prime}\left(s_{\mathrm{t}} x\right)\right] \\
& b_{n m} \psi_{n}(x)+q_{n m} \xi_{n}(x)=\beta_{n m} \tilde{\psi}_{n}\left(s_{\mathrm{t}} x\right)+\delta_{n m} \tilde{\chi}_{n}\left(s_{\mathrm{t}} x\right) .
\end{aligned}
$$

These lead to:

$$
\begin{aligned}
\Gamma_{n} & =-\frac{[\psi \psi]_{n, x}^{m, s}+\Gamma_{n}^{(2)}[\chi \psi]_{n, x}^{m, s}}{[\psi \xi]_{n, x}^{m, s}+\Gamma_{n}^{(2)}[\chi \xi]_{n, x}^{m, s}} \\
\Delta_{n} & =-\frac{[\psi \psi]_{n, x}^{e, s}+\Delta_{n}^{(2)}[\chi \psi]_{n, x}^{e, s}}{[\psi \xi]_{n, x}^{m, s}+\Delta_{n}^{(2)}[\chi \xi]_{n, x}^{e, s}}
\end{aligned}
$$

or

$$
\begin{aligned}
\Gamma_{n} & =-\frac{[\psi \psi]_{n, x}^{m, s}\left([\chi \psi]_{n, y}^{m, s}+\Gamma_{n}^{(1)}[\chi \chi]_{n, y}^{m, s}\right)-[\chi \psi]_{n, x}^{m, s}\left([\psi \psi]_{n, y}^{m, s}+\Gamma_{n}^{(1)}[\psi \chi]_{n, y}^{m, s}\right)}{[\psi \xi]_{n, x}^{m, s}\left([\chi \psi]_{n, y}^{m, s}+\Gamma_{n}^{(1)}[\chi \chi]_{n, y}^{m, s}\right)-[\chi \xi]_{n, x}^{m, s}\left([\psi \psi]_{n, y}^{m, s}+\Gamma_{n}^{(1)}[\psi \chi]_{n, y}^{m, s}\right)} \\
& \equiv-\frac{N_{1 n}}{D_{1 n}} \\
\Delta_{n} & =-\frac{[\psi \psi]_{n, x}^{e, s}\left([\chi \psi]_{n, y}^{e, s}+\Delta_{n}^{(1)}[\chi \chi]_{n, y}^{e, s}\right)-[\chi \psi]_{n, x}^{e, s}\left([\psi \psi]_{n, y}^{e, s}+\Delta_{n}^{(1)}[\psi \chi]_{n, y}^{e, s}\right)}{[\psi \xi]_{n, x}^{e, s}\left([\chi \psi]_{n, y}^{e, s}+\Delta_{n}^{(1)}[\chi \chi]_{n, y}^{e, s}\right)-[\chi \xi]_{n, x}^{e, s}\left([\psi \psi]_{n, y}^{e, s}+\Delta_{n}^{(1)}[\psi \chi]_{n, y}^{e, s}\right)} \\
& \equiv-\frac{N_{2 n}}{D_{2 n}}
\end{aligned}
$$

\section{D.2 TASA Solutions}

To begin, we may first want to look at $\Delta_{n}$. By defining $\delta=x-y=k_{\mathrm{m}} l_{s}$, we are therefore searching for the approximation in the limit $\delta \ll x, y$. Taylor expansion to the first order in $\delta$ leads to the same expressions as in Eqs. 3.33 and 3.34. Therefore, we need to find the terms $\left.N_{2 n}\right|_{x=y},\left.D_{2 n}\right|_{x=y},\left.N_{2 n}^{\prime}\right|_{x=y}$, and $\left.D_{2 n}^{\prime}\right|_{x=y}$ in order to find the TASA expression for $\Delta_{n}(\delta)$ [similar for $\Gamma_{n}(\delta)$ ]. By applying some of the useful 
identities earlier, we can derive the following:

$$
\begin{aligned}
\left.N_{2 n}\right|_{x=y}= & {[\psi \psi]_{n, y}^{e, s}\left([\chi \psi]_{n, y}^{e, s}+\Delta_{n}^{(1)}[\chi \chi]_{n, y}^{e, s}\right)-[\chi \psi]_{n, y}^{e, s}\left([\psi \psi]_{n, y}^{e, s}+\Delta_{n}^{(1)}[\psi \chi]_{n, y}^{e, s}\right) } \\
= & \Delta_{n}^{(1)}[\psi \psi]_{n, y}^{e, s}\left\{s_{\mathrm{t}} \tilde{\chi}_{n}\left(s_{\mathrm{t}} y\right) \chi_{n}^{\prime}(y)-\tilde{\chi}_{n}^{\prime}\left(s_{\mathrm{t}} y\right) \chi_{n}(y)\right\} \\
& -\Delta_{n}^{(1)}[\chi \psi]_{n, y}^{e, s}\left\{s_{\mathrm{t}} \tilde{\psi}_{n}\left(s_{\mathrm{t}} y\right) \chi_{n}^{\prime}(y)-\tilde{\psi}_{n}^{\prime}\left(s_{\mathrm{t}} y\right) \chi_{n}(y)\right\} \\
= & \Delta_{n}^{(1)}\left\{s_{\mathrm{t}} \psi_{n}(y) \chi_{n}^{\prime}(y)-s_{\mathrm{t}} \psi_{n}^{\prime}(y) \chi_{n}(y)\right\} \\
= & s_{\mathrm{t}} \Delta_{n}^{(1)} .
\end{aligned}
$$

From second to third equality, we used Eqs. 3.37 and 3.38. And the last equality the Wronskian identiy of the Riccati-Bessel function was used $\psi \chi^{\prime}-\psi^{\prime} \chi=1$, which leads to $\xi \chi^{\prime}-\xi^{\prime} \chi=1$ and $\psi \xi^{\prime}-\psi^{\prime} \xi=i$. Similarly, the denominator $\left.D_{1 n}\right|_{x=y}$ can be found as:

$$
\left.D_{1 n}\right|_{x=y}=s_{\mathrm{t}}\left(\Delta_{n}^{(1)}-i\right) .
$$

Now, if we use Eq. 3.50, we can write the derivatives of the numerator and the denominator as:

$$
\begin{aligned}
\left.N_{2 n}^{\prime}\right|_{x=y}= & {\left[\frac{s_{\mathrm{t}} n(n+1)}{y^{2}}\left(1-\frac{\varepsilon_{\mathrm{t}}}{\varepsilon_{\mathrm{n}} s_{\mathrm{t}}^{2}}\right) \tilde{\psi}_{n}\left(s_{\mathrm{t}} y\right) \psi_{n}(y)+\left(s_{\mathrm{t}}^{2}-1\right) \tilde{\psi}_{n}^{\prime}\left(s_{\mathrm{t}} y\right) \psi_{n}^{\prime}(y)\right] } \\
& \times\left([\chi \psi]_{n, y}^{e, s}+\Delta_{n}^{(1)}[\chi \chi]_{n, y}^{e, s}\right) \\
& -\left[\frac{s_{\mathrm{t}} n(n+1)}{y^{2}}\left(1-\frac{\varepsilon_{\mathrm{t}}}{\varepsilon_{\mathrm{n}} s_{\mathrm{t}}^{2}}\right) \tilde{\chi}_{n}\left(s_{\mathrm{t}} y\right) \psi_{n}(y)+\left(s_{\mathrm{t}}^{2}-1\right) \tilde{\chi}_{n}^{\prime}\left(s_{\mathrm{t}} y\right) \psi_{n}^{\prime}(y)\right] \\
& \times\left([\psi \psi]_{n, y}^{e, s}+\Delta_{n}^{(1)}[\psi \chi]_{n, y}^{e, s}\right) \\
= & \frac{s_{\mathrm{t}} n(n+1)}{y^{2}}\left(\frac{\varepsilon_{\mathrm{t}}}{\varepsilon_{\mathrm{n}} s_{\mathrm{t}}^{2}}-1\right) \psi_{n}(y)\left[\psi_{n}(y)+\Delta_{n}^{(1)} \chi_{n}(y)\right] \\
& +s_{\mathrm{t}}\left(1-s_{\mathrm{t}}^{2}\right) \psi_{n}^{\prime}(y)\left[\psi_{n}^{\prime}(y)+\Delta_{n}^{(1)} \chi_{n}^{\prime}(y)\right] .
\end{aligned}
$$

And

$$
\begin{aligned}
\left.D_{2 n}^{\prime}\right|_{x=y}= & \frac{s_{\mathrm{t}} n(n+1)}{y^{2}}\left(\frac{\varepsilon_{\mathrm{t}}}{\varepsilon_{\mathrm{n}} s_{\mathrm{t}}^{2}}-1\right) \xi_{n}(y)\left[\psi_{n}(y)+\Delta_{n}^{(1)} \chi_{n}(y)\right] \\
& +s_{\mathrm{t}}\left(1-s_{\mathrm{t}}^{2}\right) \xi_{n}^{\prime}(y)\left[\psi_{n}^{\prime}(y)+\Delta_{n}^{(1)} \chi_{n}^{\prime}(y)\right] .
\end{aligned}
$$

Similar procedures can be applied to get all the terms for $\Gamma_{n}(\delta)$, which are:

$$
\begin{aligned}
& \left.N_{1 n}\right|_{x=y}=s_{\mathrm{t}} \Gamma_{n}^{(1)} \\
& \left.D_{1 n}\right|_{x=y}=s_{\mathrm{t}}\left(\Gamma_{n}^{(1)}-i\right) \\
& \left.N_{1 n}^{\prime}\right|_{x=y}=s_{\mathrm{t}}\left(s_{\mathrm{t}}^{2}-1\right) \psi_{n}(y)\left[\psi_{n}(y)+\Gamma_{n}^{(1)} \chi_{n}(y)\right] \\
& \left.D_{1 n}^{\prime}\right|_{x=y}=s_{\mathrm{t}}\left(s_{\mathrm{t}}^{2}-1\right) \xi_{n}(y)\left[\psi_{n}(y)+\Gamma_{n}^{(1)} \chi_{n}(y)\right] .
\end{aligned}
$$




\section{Bibliography}

[1] P. M. Carvalho, M. R. Felício, N. C. Santos, S. Gonçalves, and M. M. Domingues. Application of Light Scattering Techniques to Nanoparticle Characterization and Development. Front. Chem, 6:237, 2018.

[2] C. Fornaguera and C. Solans. Characterization of Polymeric Nanoparticle Dispersions for Biomedical Applications: Size, Surface Charge and Stability. Pharm. Nanotechnol., 6(3):147-164, 2018.

[3] M. Min, J. W. Hovenier, and A. de Koter. Modeling Optical Properties of Cosmic Dust Grains Using a Distribution of Hollow Spheres. Astron. Astrophys., 432(3):909-920, 2005.

[4] N. V. Tkachenko. Optical Spectroscopy: Methods and Instrumentations. Elsevier, 2006.

[5] W. W. Parson. Modern Optical Spectroscopy, volume 2. Springer, 2007.

[6] J. N. Anker, W. P. Hall, O. Lyandres, N. C. Shah, J. Zhao, and R. P. Van Duyne. Biosensing with Plasmonic Nanosensors. Nanoscience and Technology: A Collection of Reviews from Nature Journals, pages 308-319, 2010.

[7] W. C. Law, K. T. Yong, A. Baev, and P. N. Prasad. Sensitivity Improved Surface Plasmon Resonance Biosensor for Cancer Biomarker Detection Based on Plasmonic Enhancement. ACS Nano, 5(6):4858-4864, 2011.

[8] S. Zhang, R. Geryak, J. Geldmeier, S. Kim, and V. V. Tsukruk. Synthesis, Assembly, and Applications of Hybrid Nanostructures for Biosensing. Chem. Rev., 117(20):12942-13038, 2017.

[9] B. S. Hoener, S. R. Kirchner, T. S. Heiderscheit, S. S. Collins, W. S. Chang, S. Link, and C. F. Landes. Plasmonic Sensing and Control of SingleNanoparticle Electrochemistry. Chem., 4(7):1560-1585, 2018. 
[10] T. W. Odom and G. C. Schatz. Introduction to Plasmonics. Chem. Rev., 111(6):3667-3668, 2011.

[11] I. S. Maksymov, I. Staude, A. E. Miroshnichenko, and Y. S. Kivshar. Optical Yagi-Uda Nanoantennas. Nanophotonics, 1(1):65-81, 2012.

[12] T. Kosako, Y. Kadoya, and H. F. Hofmann. Directional Control of Light by a Nano-Optical Yagi-Uda Antenna. Nat. Photonics, 4(5):312, 2010.

[13] O. Hess, J. B. Pendry, S. A. Maier, R. F. Oulton, J. M. Hamm, and K. L. Tsakmakidis. Active Nanoplasmonic Metamaterials. Nat. Mater., 11(7):573, 2012.

[14] N. Engheta. Circuits with Light at Nanoscales: Optical Nanocircuits Inspired by Metamaterials. Science, 317(5845):1698-1702, 2007.

[15] S. Kühn, U. Håkanson, L. Rogobete, and V. Sandoghdar. Enhancement of Single-Molecule Fluorescence using a Gold Nanoparticle as an Optical Nanoantenna. Phys. Rev. Lett., 97(1):017402, 2006.

[16] C. M. Galloway, P. G. Etchegoin, and E. C. Le Ru. Ultrafast Nonradiative Decay Rates on Metallic Surfaces by Comparing Surface-Enhanced Raman and Fluorescence Signals of Single Molecules. Phys. Rev. Lett., 103(6):063003, 2009.

[17] R. Gill and E. C. Le Ru. Fluorescence Enhancement at Hot-Spots: The Case of Ag Nanoparticle aggregates. Phys. Chem. Chem. Phys., 13(36):16366-16372, 2011.

[18] R. Gill, L. Tian, W. R. C. Somerville, E. C. Le Ru, H. van Amerongen, and V. Subramaniam. Silver Nanoparticle Aggregates as Highly Efficient Plasmonic Antennas for Fluorescence Enhancement. J. Phys. Chem. C, 116(31):1668716693, 2012.

[19] A. Bek, R. Jansen, M. Ringler, S. Mayilo, T. A. Klar, and J. Feldmann. Fluorescence Enhancement in Hot Spots of AFM-Designed Gold Nanoparticle Sandwiches. Nano Lett., 8(2):485-490, 2008.

[20] E. C. Le Ru and P. G. Etchegoin. Single-Molecule Surface-Enhanced Raman Spectroscopy. Annu. Rev. Phys. Chem., 63:65-87, 2012.

[21] J. Qi, X. Dang, P. T. Hammond, and A. M. Belcher. Highly Efficient PlasmonEnhanced Dye-Sensitized Solar Cells through Metal@ Oxide Core-Shell Nanostructure. ACS Nano, 5(9):7108-7116, 2011. 
[22] O. L. Muskens, J. G. Rivas, R. E. Algra, E. P. Bakkers, and A. Lagendijk. Design of Light Scattering in Nanowire Materials for Photovoltaic Applications. Nano Lett., 8(9):2638-2642, 2008.

[23] W. Ni, T. Ambjornsson, S. P. Apell, H. Chen, and J. Wang. Observing Plasmonic- Molecular Resonance Coupling on Single Gold Nanorods. Nano Lett., 10(1):77-84, 2010.

[24] W. Ni, H. Chen, J. Su, Z. Sun, J. Wang, and H. Wu. Effects of Dyes, Gold Nanocrystals, pH, and Metal Ions on Plasmonic and Molecular Resonance Coupling. J. Am. Chem. Soc., 132(13):4806-4814, 2010.

[25] J. Zhao, L. Jensen, J. Sung, S. Zou, G. C. Schatz, and R. P. Van Duyne. Interaction of Plasmon and Molecular Resonances for Rhodamine 6G Adsorbed on Silver Nanoparticles. J. Am. Chem. Soc., 129(24):7647-7656, 2007.

[26] J. Yan, W. Zhang, S. Duan, X. Zhao, and A. O. Govorov. Optical Properties of Coupled Metal-Semiconductor and Metal-Molecule Nanocrystal Complexes: Role of Multipole Effects. Phys. Rev. B, 77(16):165301, 2008.

[27] E. C. Le Ru and P. G. Etchegoin. Principles of Surface-Enhanced Raman Spectroscopy: and Related Plasmonic Effects. Elsevier, 2009.

[28] N. T. Fofang, T. Park, O. Neumann, N. A. Mirin, P. Nordlander, and N. J. Halas. Plexcitonic Nanoparticles: Plasmon- Exciton Coupling in NanoshellJ-Aggregate Complexes. Nano Lett., 8(10):3481-3487, 2008.

[29] T. J. Antosiewicz, S. P. Apell, and T. Shegai. Plasmon-Exciton Interactions in a Core-Shell Geometry: from Enhanced Absorption to Strong Coupling. ACS Photonics, 1(5):454-463, 2014.

[30] A. O. Cacciola, O. Di Stefano, R. Stassi, R. Saija, and S. Savasta. Ultrastrong Coupling of Plasmons and Excitons in a Nanoshell. ACS Nano, 8(11):1148311492, 2014.

[31] A. M. Fales, S. J. Norton, B. M. Crawford, B. G. DeLacy, and T. Vo-Dinh. Fano Resonance in a Gold Nanosphere with a J-Aggregate Coating. Phys. Chem. Chem. Phys., 17(38):24931-24936, 2015.

[32] R. Chikkaraddy, B. de Nijs, F. Benz, S. J. Barrow, O. A. Scherman, E. Rosta, A. Demetriadou, P. Fox, O. Hess, and J. J Baumberg. Single-Molecule 
Strong Coupling at Room Temperature in Plasmonic Nanocavities. Nature, 535(7610):127, 2016.

[33] G. Zengin, T. Gschneidtner, R. Verre, L. Shao, T. J. Antosiewicz, K. MothPoulsen, M. Käll, and T. Shegai. Evaluating Conditions for Strong Coupling Between Nanoparticle Plasmons and Organic Dyes using Scattering and Absorption Spectroscopy. J. Phys. Chem. C, 120(37):20588-20596, 2016.

[34] B. L. Darby, B. Auguié, M. Meyer, A. E. Pantoja, and E. C. Le Ru. Modified Optical Absorption of Molecules on Metallic Nanoparticles at Sub-Monolayer Coverage. Nat. Photonics, 10(1):40, 2016.

[35] B. Auguié and E. C. Le Ru. Optical Absorption of Dye Molecules in a Spherical Shell Geometry. J. Phys. Chem. C, 122(33):19110-19115, 2018.

[36] E. M. Purcell and C. R. Pennypacker. Scattering and Absorption of Light by Nonspherical Dielectric Grains. Astrophys. J., 186:705-714, 1973.

[37] B. Auguié, B. L. Darby, and E. C. Le Ru. Electromagnetic Interactions of Dye Molecules Surrounding a Nanosphere. Nanoscale, 2019.

[38] C. F. Bohren and D. R. Huffman. Absorption and Scattering of Light by Small Particles. John Wiley \& Sons, 2008.

[39] S. Teitler and B. W. Henvis. Refraction in Stratified, Anisotropic Media. J. Opt. Soc. Am., 60(6):830-834, 1970.

[40] J. Roth and M. J. Dignam. Scattering and Extinction Cross Sections for a Spherical Particle Coated with an Oriented Molecular Layer. J. Opt. Soc. Am., 63(3):308-311, 1973.

[41] G. C. Papavassiliou. Optical Properties of Small Inorganic and Organic Metal Particles. Prog. Solid State Chem., 12(3-4):185-271, 1979.

[42] C. W. Qiu, L. Gao, J. D. Joannopoulos, and M. Soljačić. Light Scattering from Anisotropic Particles: Propagation, Localization, and Nonlinearity. Laser \&6 Photonics Rev., 4(2):268-282, 2010.

[43] H. Wallén, H. Kettunen, and A. Sihvola. Anomalous Absorption, Plasmonic Resonances, and Invisibility of Radially Anisotropic Spheres. Radio Sci., 50(1):18-28, 2015. 
[44] M. J. Dignam and M. Moskovits. Optical Properties of Sub-Monolayer Molecular Films. J. Chem. Soc. Faraday Trans 2: Molec. Chem. Phys., 69:56-64, 1973.

[45] A. Bagchi, R. G. Barrera, and B. B. Dasgupta. Classical Local-Field Effect in Reflectance from Adsorbed Overlayers. Phys. Rev. Lett., 44(22):1475, 1980.

[46] A. Bagchi, R. G. Barrera, and R. Fuchs. Local-Lield Effect in Optical Reflectance from Adsorbed Overlayers. Phys. Rev. B, 25(12):7086, 1982.

[47] C. Tang, B. Auguié, and E. C. Le Ru. Refined Effective-Medium Model for the Optical Properties of Nanoparticles Coated with Anisotropic Molecules. Phys. Rev. B, 103(8):085436, 2021.

[48] C. Tang, B. Auguié, and E. C. Le Ru. Modeling Molecular Orientation Effects in Dye-Coated Nanostructures Using a Thin-Shell Approximation of Mie Theory for Radially Anisotropic Media. ACS Photonics, 5(12):5002-5009, 2018.

[49] M. I. Mishchenko, L. D. Travis, and A. A. Lacis. Scattering, Absorption, and Emission of Light by Small Particles. Cambridge University Press, 2002.

[50] P. Monk. Finite Element Methods for Maxwell's Equations. Oxford University Press, 2003.

[51] A. Taflove and S. C. Hagness. Computational Electrodynamics: the FiniteDifference Time-Domain Method. Artech House, Boston, 2005.

[52] M. Abramowitz and I. A. Stegun. Handbook of Mathematical Functions with Formulas, Graphs, and Mathematical Tables. National Bureau of Standards Applied Mathematics Series 55. Tenth Printing. 1972.

[53] R. D. Averitt, D. Sarkar, and N. J. Halas. Plasmon Resonance Shifts of AuCoated $\mathrm{Au}_{2} \mathrm{~S}$ Nanoshells: Insight into Multicomponent Nanoparticle Growth. Phys. Rev. Lett., 78(22):4217, 1997.

[54] E. Prodan and P. Nordlander. Structural Tunability of the Plasmon Resonances in Metallic Nanoshells. Nano Lett., 3(4):543-547, 2003.

[55] X. Zhong, S. G. Rodrigo, L. Zhang, P. Samorì, C. Genet, L. Martín-Moreno, J. A. Hutchison, and T. W. Ebbesen. Waveguide and Plasmonic AbsorptionInduced Transparency. ACS Nano, 10(4):4570-4578, 2016. 
[56] H. Wang, H.-Y. Wang, A. Bozzola, A. Toma, S. Panaro, W. Raja, A. Alabastri, L. Wang, Q. D. Chen, H. L. Xu, and F. De Angelis. Dynamics of Strong Coupling between J-Aggregates and Surface Plasmon Polaritons in Subwavelength Hole Arrays. Adv. Funct. Mater., 26(34):6198-6205, 2016.

[57] B. Lange and S. R. Aragón. Mie Scattering from Thin Anisotropic Spherical Shells. J. Chem. Phys., 92(8):4643-4650, 1990.

[58] D. K. Hahn and S. R. Aragón. Mie Scattering from Anisotropic Thick Spherical Shells. J. Chem. Phys., 101(10):8409-8417, 1994.

[59] M. Moskovits. Surface Selection Rules. J. Chem. Phys., 77(9):4408-4416, 1982.

[60] M. Moskovits. Surface-Enhanced Spectroscopy. Rev. Mod. Phys., 57(3):783, 1985.

[61] E. C. Le Ru, S. A. Meyer, C. Artur, P. G. Etchegoin, J. Grand, P. Lang, and F. Maurel. Experimental Demonstration of Surface Selection Rules for SERS on Flat Metallic Surfaces. Chem. Commun., 47(13):3903-3905, 2011.

[62] D. E. Aspnes. Optical Properties of Thin Films. Thin Solid Films, 89(3):249$262,1982$.

[63] V. A. Markel. Introduction to the Maxwell Garnett Approximation: Tutorial. J. Opt. Soc. Am. A, 33(7):1244-1256, 2016.

[64] E. C. Le Ru and P. G. Etchegoin. Matlab codes freely available from. SERS and Plasmonics Codes (SPlaC). http://www.vuw.ac.nz/raman/book/codes.aspx.

[65] A. Djorovic̀, M. Meyer, B. L. Darby, and E. C. Le Ru. Accurate Modeling of the Polarizability of Dyes for Electromagnetic Calculations. ACS Omega, 2(5):1804-1811, 2017.

[66] H. DeVoe. Optical Properties of Molecular Aggregates. I. Classical Model of Electronic Absorption and Refraction. J. Chem. Phys., 41(2):393-400, 1964.

[67] H. DeVoe. Optical Properties of Molecular Aggregates. II. Classical Theory of the Refraction, Absorption, and Optical Activity of Solutions and Crystals. J. Chem. Phys., 43(9):3199-3208, 1965.

[68] N. C. Maiti, S. Mazumdar, and N. Periasamy. J-and H-Aggregates of PorphyrinSurfactant Complexes: Time-Resolved Fluorescence and Other Spectroscopic Studies. J. Phys. Chem. B, 102(9):1528-1538, 1998. 
[69] D. E. Aspnes. Local-field effects and effective-medium theory: a microscopic perspective. Am. J. Phys., 50(8):704-709, 1982.

[70] F. H. P. M. Habraken, O. L. J. Gijzeman, and G. A. Bootsma. Ellipsometry of Clean Surfaces, Submonolayer and Monolayer Films. Surf. Sci., 96(1-3):482$507,1980$.

[71] J. Lekner and P. J. Castle. Local Fields near the Surface of a Crystalline Dielectric. Physica A: Stat. Mech. Appl., 101(1):89-98, 1980.

[72] J. Vlieger. Reflection and Transmission of Light by a Square Non-Polar Lattice. Physica, 64(1):63-81, 1973.

[73] G. W. Ford and W. H. Weber. Electromagnetic Interactions of Molecules with Metal Surfaces. Phys. Rep., 113(4):195-287, 1984.

[74] C. Tang, B. Auguié, and E. C. Le Ru. Thin-shell approximation of Mie theory for a thin anisotropic layer spaced away from a spherical core: Application to dye-coated nanostructures. Phys. Rev. A, (in press), 2021.

[75] B. T. Draine and P. J. Flatau. Discrete-Dipole Approximation for Scattering Calculations. J. Opt. Soc. Am. A, 11(4):1491-1499, 1994.

[76] M. I. Mishchenko, J. W. Hovenier, and L. D. Travis. Light Scattering by Nonspherical Particles: Theory, Measurements, and Applications, 2000.

[77] J. Parsons, C. P. Burrows, J. R. Sambles, and W. L. Barnes. A Comparison of Techniques used to Simulate the Scattering of Electromagnetic Radiation by Metallic Nanostructures. J. Mod. Opt., 57(5):356-365, 2010.

[78] M. A. Yurkin. Computational Approaches for Plasmonics. Handbook of Molecular Plasmonics, pages 83-135, 2013.

[79] J. Grand and E. C. Le Ru. Practical Implementation of Accurate FiniteElement Calculations for Electromagnetic Scattering by Nanoparticles. Plasmonics, 15(1):109-121, 2020.

[80] X. Huang, P. K. Jain, I. H. El-Sayed, and M. A. El-Sayed. Plasmonic Photothermal Therapy (PPTT) Using Gold Nanoparticles. Lasers Med. Sci., 23(3):217, 2008.

[81] W. Haiss, N. T. K. Thanh, J. Aveyard, and D. G. Fernig. Determination of Size and Concentration of Gold Nanoparticles from UV- Vis Spectra. Anal. Chem., 79(11):4215-4221, 2007. 
[82] P. G. Etchegoin, E. C. Le Ru, and M. Meyer. An Analytic Model for the Optical Properties of Gold. J. Chem. Phys., 125(16):164705, 2006.

[83] K. Aslan, Z. Leonenko, J. R. Lakowicz, and C. D Geddes. Fast and Slow Deposition of Silver Nanorods on Planar Surfaces: Application to Metal-Enhanced Fluorescence. J. Phys. Chem. B, 109(8):3157-3162, 2005.

[84] A. L. Schmucker, N. Harris, M. J. Banholzer, M. G. Blaber, K. D. Osberg, G. C. Schatz, and C. A. Mirkin. Correlating Nanorod Structure with Experimentally Measured and Theoretically Predicted Surface Plasmon Resonance. ACS Nano, 4(9):5453-5463, 2010.

[85] E. C. Le Ru, J. Grand, I. Sow, W. R. C. Somerville, P. G. Etchegoin, M. TreguerDelapierre, G. Charron, N. Félidj, G. Lévi, and J. Aubard. A Scheme for Detecting Every Single Target Molecule with Surface-Enhanced Raman Spectroscopy. Nano Lett., 11(11):5013-5019, 2011.

[86] E. C. Le Ru and P. G. Etchegoin. Matlab codes freely available from. SMARTIES. http://www.vuw.ac.nz/raman/book/codes.aspx.

[87] T. R. Jensen, M. L. Duval, K. L. Kelly, A. A. Lazarides, G. C. Schatz, and R. P. Van Duyne. Nanosphere Lithography: Effect of the External Dielectric Medium on the Surface Plasmon Resonance Spectrum of a Periodic Array of Silver Nanoparticles. J. Phys. Chem. B, 103(45):9846-9853, 1999.

[88] M. Lattuada and T. A. Hatton. Synthesis, Properties and Applications of Janus Nanoparticles. Nano Today, 6(3):286-308, 2011.

[89] S. J. de Carvalho, R. Metzler, and A. G. Cherstvy. Inverted Critical Adsorption of Polyelectrolytes in Confinement. Soft Matter, 11(22):4430-4443, 2015.

[90] M. Abramowitz and I. A. Stegun. Handbook of Mathematical Functions: with Formulas, Graphs, and Mathematical Tables, volume 55. Courier Corporation, 1965. 